\title{
Monkey see, monkey touch, monkey do: Influence of visual and tactile input on the fronto-parietal grasping network
}

\author{
Dissertation \\ for the award of the degree \\ "Doctor rerum naturalium" \\ of the Georg-August-Universität Göttingen
}

within the doctoral program Sensory and Motor Neuroscience of the Georg-August University School of Science (GAUSS)

Submitted by

Daniela Buchwald

from Helmstedt, Germany

Göttingen 2020 


\section{Thesis Committee}

\section{Referee:}

Prof. Dr. Hansjörg Scherberger

Neurobiology Laboratory

Deutsches Primatenzentrum

Kellnerweg 4, 37077 Göttingen

\section{2nd Referee:}

Dr. Igor Kagan

Decision and Awareness Group

Deutsches Primatenzentrum

Kellnerweg 4, 37077 Göttingen

Prof. Dr. Ralf Heinrich

Cellular Neurobiology

Schwann-Schleiden Research Centre

Julia-Lermontowa-Weg 3, 37077 Göttingen

\section{Members of the Examination Board}

Prof. Dr. Manuela Schmidt

Somatosensory Signaling Group

Max Planck Institute for Experimental Medicine

Hermann-Rein-Str. 3, 37075 Göttingen

Prof. Dr. Tim Gollisch

Sensory Processing in the Retina

University Medical Center Göttingen

Waldweg 33, 37073 Göttingen

Prof. Dr. Alexander Gail

Sensorimotor Group

Deutsches Primatenzentrum

Kellnerweg 4, 37077 Göttingen

Date of oral examination: $13^{\text {th }}$ of March, 2020 
Today, so many moons away, I safely say that my heart's true calling was never betrayed

Arrays of enemies await, but fears allay, We stand as one, a bond beyond the vast wave until the last grave - TWRP, Starlight Brigade 


\section{Contents}

Abstract vii

Zusammenfassung ix

List of Figures $\quad$ XV

Abbreviations $\quad$ xv

1 Introduction 1

1.1 Overview over the planning and execution of grasping $\ldots \ldots \ldots$

1.1.1 Processing of visual information in the anterior intraparietal cortex 3

1.1.2 The importance of the somatosensory system for grasping . . . . 4

1.1.3 The premotor cortex and the planning of movements . . . . . . 9

1.1.4 Generation of movements in the primary motor cortex . . . . . . 9

1.2 Introduction to neural interfaces . . . . . . . . . . . . . 12

1.3 Motivation and objectives of this thesis . . . . . . . . . . . . . 14

2 Materials and Methods 17

2.1 subjects ............................. 17

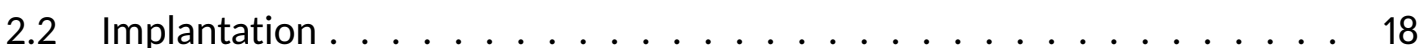

2.3 Neural recordings . . . . . . . . . . . . . . . . . . . 22

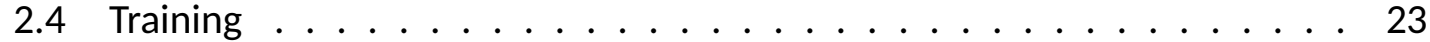

2.5 Delayed-grasping or object recognition task . . . . . . . . . . . . . 24

$2.5 .1 \quad$ Experimental setup . . . . . . . . . . . . 25 
2.5 .2 Objects on turntable ............... 26

2.5.3 Recording of hand kinematics . . . . . . . . . . . . . 27

2.5.4 Behavioural paradigm . . . . . . . . . . . 30

2.6 Passive stimulation experiment . . . . . . . . . . . . . . . . . 31

2.6.1 Experimental setup and behavioural paradigm . . . . . . . 31

2.6 .2 Stimuli ...................... . . 34

2.7 Data analysis . . . . . . . . . . . . . . . 36

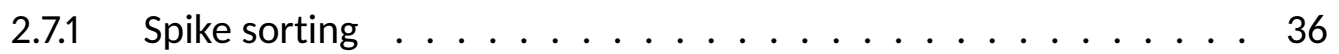

2.7.2 Movement time analysis . . . . . . . . . . . . 36

2.7 .3 Peristimulus time histogram . . . . . . . . . . . . . 38

2.7.4 Population tuning . . . . . . . . . . . . . . . . . . 41

2.7.5 Receiver operating characteristic . . . . . . . . . . . . . . 42

2.7.6 Decoding ....................... 43

$\begin{array}{lll}3 & \text { Results } & 47\end{array}$

3.1 Number of units per recording day . . . . . . . . . . . . . . . . . . 47

3.2 Visual and tactile object recognition $\ldots \ldots \ldots$. . . . . . . . . 48

3.3 Grasping psychophysics . . . . . . . . . . . . . . . . . . . . . . 48

3.3.1 Movement and reaction times for grasps during visual and tactile trials were similar while tactile exploration took longer . . . . . 50

3.3.2 Unit activity varied per area . . . . . . . . . . . . 53

3.3.2.1 Strong motor activity in area F5 . . . . . . . . 53

3.3.2.2 Strong motor activity in area $\mathrm{M} 1 \ldots \ldots$. . . . . . 55

3.3.2.3 Strong correlation with presence of tactile input in area S1 58

3.3.2.4 Unit activity in area AIP . . . . . . . . . . . 59

3.3.3 Population tuning revealed differences in activity between visual and tactile trials in the memory epoch . . . . . . . . . . . 60

3.3.4 ROC analysis revealed tuning towards sensory condition in all areas 63 
3.3.4.1 ROC analysis for premotor cortex revealed better discriminability during early memory . . . . . . . . 64

3.3.4.2 ROC scores of primary motor cortex were higher during early memory ............. . . 65

3.3.4.3 ROC scores of primary somatosensory decreases over time .................... 67

3.3.4.4 ROC scores of anterior intraparietal cortex were best during early memory . . . . . . . . . . 68

3.3 .5 Decoding ......................... 70

3.4 Passive finger stimulation experiment $\ldots \ldots \ldots$. . . . . . . . 75

3.4.1 Single neuron activity . . . . . . . . . . . 75

3.4.1.1 No strong correlation with elements of the task in area

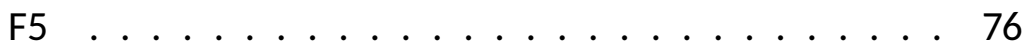

3.4.1.2 Units in area AIP showed only minor changes during stimulation . . . . . . . . . . . . 77

3.4.1.3 Units in $\mathrm{M} 1$ reacted to tactile stimulation . . . . . . 79

3.4.1.4 Tactile stimulation drove the activity of $\mathrm{S} 1$ units $\ldots 81$

3.4.2 Population analysis revealed tactile tuning in S1 and M1 and no tuning in AlP and $\mathrm{F} 5 \ldots \ldots \ldots . \ldots . \ldots 3$

4 Discussion $\quad 87$

4.1 Visual and tactile object recognition $\ldots \ldots \ldots$. . . . . . . 88

4.1.1 The animal uses cue information to determine the right grasp type 88

4.1.2 Visual and tactile trials elicit a slightly different memory activity . 89

4.1.3 Differences in the encoding of objects . . . . . . . . . . . 992

4.2 Passive finger stimulation . . . . . . . . . . . . . . . . . . 94

4.2.1 During passive finger stimulation, tactile stimulation elicits responses in S1 and M1, but not in AIP and F5 . . . . . . . . . . 95

4.3 Tactile input in the different brain areas $\ldots \ldots \ldots 96$

4.3.1 Motor cortex responded to tactile input as well as active movements of the animal . . . . . . . . . . . . 96 
4.3.2 In both tasks the somatosensory cortex reacted to tactile stimuli . 97

4.3.3 The anterior intraparietal area shows no modulation to tactile input 98

4.3.4 Premotor cortex area F5 responded to movements of the animal but not passive tactile inputs . . . . . . . . . . . . 99

5 Conclusion 101

6 References 103

References . . . . . . . . . . . . . . . . . . 103

$\begin{array}{lll}7 & \text { Acknowledgements } & 117\end{array}$ 


\section{Abstract}

One of the most common movements we do every day is grasping. Usually we do this with aid of our eyes, but other senses, like our sense of touch, can also assist in the preparation and execution of grasping movements. More so, constant feedback is essential when interacting with objects in order to react and adapt to changing situations, such as object slippage. While it is generally assumed that different brain areas are responsible for different types of input or tasks (although there is no perfect separation, most areas process different types of information) each movement is a team effort of many different areas. These interactions between brain areas in order to generate movements have not yet been extensively studied, especially not when the object information is delivered by different senses.

In this thesis I investigated how tactile input is processed by different brain areas and how this information is used to plan and generate grasping movements. More so, I studied whether or not the planning and generation of grasping movements in the brain differs when based on visual compared to tactile information. For this purpose multi-electrode arrays were implanted into the primary motor cortex (M1), primary somatosensory cortex (S1), anterior intraparietal area (AIP) and the hand area of the ventral premotor cortex (area F5) of a rhesus macaque (Macaca mulatta).

The animal was trained to grasp objects that he either saw or touched beforehand, allowing me to compare how information from both conditions is processed in the brain. By comparing firing rates, differences in the brain activity between both conditions were found. When looking at the number of significantly tuned neurons, it is obvious that there are differences in the way the brain plans grasping movements on the basis of vision versus touch, while the actual execution does not differ greatly. When trying to decode which trials were visually or tactually guided, accuracy is best in early memory but differentiation between both conditions is still possible shortly before grasping. In a second experiment, passive finger stimulation was applied to the middle finger of the animal, giving some insight in how tactile information is processed in the four brain areas. Together this demonstrates the influence of tactile input on the fronto-parietal grasping circuit. AIP, an area known to process visual object information and F5, known for grasp 
preparation, both show no reaction to tactile input in the absence of grasp intentions. More importantly, all four areas show significant differences in memory activity between visual and tactile grasps, enough to be able to decode both conditions from neuronal activity, showing that considerations how object information was acquired might be needed when trying to record from the fronto-parietal grasping network in order to control prosthetic robot arms. 


\section{Zusammenfassung}

Einige der häufigsten Bewegungen die wir täglich ausführen sind Greifbewegungen. Normalerweise greifen wir Objekte, nachdem wir sie gesehen haben. Doch auch andere Sinne, wie unser Tastsinn, können uns bei der Vorbereitung und Ausführung von Greifbewegungen behilflich sein. Tatsächlich ist ständiges Feedback im Umgang mit Objekten essentiell: Es hilft uns dabei, uns auf wechselnde Situationen einzustellen, zum Beispiel wenn ein Objekt aus unserer Hand zu rutschen droht. Obwohl generell angenommen

wird, dass verschiedene Gehirnbereiche für verschiedene Aufgaben oder Sinne zuständig sind (wobei es keine komplette Trennung gibt, da die meisten Bereiche verschiedene Arten von Informationen verarbeiten) ist jede Bewegung ein Ergebnis des Zusammenspiels verschiedener Gehirnbereiche. Diese Zusammenarbeit zwischen Gehirnbereichen um Bewegungen entstehen zu lassen, wurde noch nicht umfassend erforscht, insbesondere nicht, wenn Objekte mit verschiedenen Sinnen wahrgenommen werden.

In dieser Doktorarbeit habe ich untersucht, wie Informationen, die von unserem Tastsinn (so genannte taktile Informationen) stammen in verschiedenen Gehirnbereichen verarbeitet werden und wie diese Informationen anschließend genutzt wird, um Greifbewegungen zu planen und auszuführen. Desweiteren habe ich untersucht, ob die Art und Weise wie diese Bewegungen vom Gehirn geplant und ausgeführt werden sich verändert, wenn die Planung auf visuellen oder taktilen Informationen basiert. Hierfür wurden Mehrfachelektrodenarrays in den primären Motorkortex (M1), den primären somatosensorischen Kortex (S1), das anteriore intraparietale Areal AIP und den Handbereich des ventralen Premotorkortexes (Areal F5) eines Rhesusaffen (M. mulatta) implantiert.

Der Affe wurde darauf trainiert, Objekte die er entweder gesehen oder berührt hat zu greifen. Das erlaubte es mir zu vergleichen, wie Informationen beider Bedingungen vom Gehirn verarbeitet werden. Bei einem Vergleich von Feuerraten wurden bereits Unterschiede in der Gehirnaktivität zwischen beiden Bedingungen sichtbar. Wenn man die Anzahl der signifikant modulierten Neurone vergleicht, ist es offensichtlich, dass Unterschiede in der Planung von Greifbewegungen basierend auf visuellen und taktilen Informationen vorliegen, die während der Ausführung der Bewegung nicht mehr vorhanden sind. Anschließend wurde ein Dekodierer auf den vorliegenden Daten trainiert, um 
vorhersagen zu können, welche Bewegungen auf der visuellen oder der taktilen Information basierten. Die Genauigkeit dieses Dekodierer ist während der frühen Memory-Period am höchsten. Trotzdem können auch zu einem späteren Zeitpunkt, kurz bevor die Greifbewegung beginnt, noch beide Bedingungen voneinander unterschieden werden. In einem zweiten Versuch wurde der Mittelfinger des Affen passiv stimuliert. Dies gab mir Einblicke wie taktile Information in den vier Gehirnbereichen verarbeitet wird.

Zusammen ergeben die Ergebnisse einen Eindruck, wie taktile Informationen die frontoparietalen Greifareale beeinflussen. AIP, ein Bereich, der dafür bekannt ist visuelle Objektinformationen zu verarbeiten, und F5, bekannt als Bereich für die Greifplanung, zeigen keine Reaktion zu taktilen Informationen, wenn keine Greifbewegung ausgeführt werden soll. Wichtiger noch, alle vier Bereiche zeigen signifikante Unterschiede in der neuronalen Aktivität während der Vorbereitung von Bewegungen, wenn visuell- und taktil-geleitete Bewegungen verglichen werden. Die Unterschiede sind stark genug, um die verschiedenen Bedingungen mit Hilfe eines Dekodierer unterscheiden zu können. Dies zeigt, dass die Art und Weise wie Objektinformation erworben wird berücksichtigt werden muss, wenn versucht wird, Aufnahmen der Gehirnaktivität in den fronto-parietalen Greifarealen zu machen, um damit beispielsweise eine Prothese oder einen Roboterarm zu steuern. 


\section{List of Figures}

1.1 The fronto-parietal grasping circuit in the macaque brain. . . . . . . . . 3

1.2 The sensory homunculus, representing primary motor cortex. . . . . . . 5

1.3 The fronto-parietal grasping circuit including $\mathrm{S} 1$ in the macaque brain . . 6

1.4 Multiple somatotopic representations on Brodmann areas 1, 2, 3a, 3b and 5 L. . . . . . . . . . . . . . . . . . . 7

1.5 The motor homunculus, representing the primary motor cortex. . . . . . 10

1.6 An example movement elicited by stimulation of primary motor cortex . 11

1.7 Illustration of different methods to record brain activity. . . . . . . . . . 12

1.8 Comparison of spacial and temporal resolution of different recording methods. ............................ 13

$2.1 \quad$ Images of the two monkeys. . . . . . . . . . . . . . . . . 18

2.2 Picture of a floating microelectrode array. . . . . . . . . . . . . . . . 19

2.3 Overview over the implantation process. . . . . . . . . . . . . . . . . 21

2.4 Position of arrays in monkey Ralph. . . . . . . . . . . . . . . . 22

2.5 Picture of a manual clicker used for clicker training. . . . . . . . . . 23

2.6 Front view of the turntable setup. . . . . . . . . . . . . . . . 25

2.7 Top view of the turntable setup. . . . . . . . . . . . . . . . . 26

2.83 D renders of the objects on the turntable $\ldots \ldots \ldots$. . . . . . . . . . 27

2.9 Illustration of the kinematic data glove. . . . . . . . . . . . . . . . 28

2.10 Photos of the kinematic data glove, worn by monkey Ralph. . . . . . . . 29

2.11 Illustration of the task paradigm. . . . . . . . . . . . . 30

2.12 Illustration of an monkey working on the turntable. . . . . . . . . . 31 
2.13 Image of the moveable platform used in the passive stimulation task. . . 32

2.14 Illustration of the passive stimulation task . . . . . . . . . . . . 33

2.15 Photo of the hand holder. . . . . . . . . . . . . . . 33

2.16 Close-up of one stimulation plate used for the passive stimulation task. . 34

2.17 Photos of the stimulation plates used for the passive stimulation task. . . 35

2.18 Example illustration of three stimulus plates. . . . . . . . . . . 35

2.19 Example comparison of movement times. . . . . . . . . . . . . . . 37

2.20 Example PSTH of an unit in primary motor cortex during active grasping task. . . . . . . . . . . . . . . . . . 38

2.21 Example PSTH of a multi unit in primary somatosensory cortex during passive stimulation task. . . . . . . . . . . . . . . . . . 40

2.22 Example population tuning analysis for all areas. . . . . . . . . . . . . 41

2.23 Example distribution of ROC scores in one area during the active grasping task. . . . . . . . . . . . . . . . . . 42

2.24 One example confusion matrix for decoding of active grasping trials. . . . 43

2.25 Example confusion matrices for decoding of active grasping trials in three different epochs. . . . . . . . . . . . . . . . . . . . 444

2.26 Example decoding accuracy over time. . . . . . . . . . . . . . . . . . 44

3.1 Illustration of grasp correction during tactile exploration. . . . . . . . . . 49

3.2 Grasp during tactile grasping. . . . . . . . . . . . . . . . . . . . . 49

3.3 Reaction times during active grasping task. . . . . . . . . . . . . 51

3.4 Movement times during active grasping task. . . . . . . . . . . . . 52

3.5 Firing rate of an $\mathrm{F} 5$ unit, reacting to movements. . . . . . . . . 53

3.6 Firing rate of an F5 unit showing some additional activity increase during visual cue. . . . . . . . . . . . . . . . . . . . . . . 54

3.7 Firing rate of an $\mathrm{M} 1$ unit, reacting to movements. . . . . . . . . . 55

3.8 Firing rate of an M1 unit showing additional activity increase during visual cue. ........................... 56

3.9 Firing rate of an $\mathrm{M} 1$ unit showing some unusual activity pattern. . . . . . 57 
3.10 Firing rate of an $\mathrm{S} 1 \mathrm{unit}$, corresponding with tactile input. . . . . . . . . 58

3.11 Firing rate of an $\mathrm{S} 1$ unit, also corresponding with tactile input. . . . . . . 59

3.12 Firing rate of an AIP unit, mainly active during tactile exploration and grasping. . . . . . . . . . . . . . . . . 60 60

3.13 Number of significant units (factor objects) at each time point for visual trials during active grasping experiment. . . . . . . . . . . 61

3.14 Number of significant units (factor objects) at each time point for tactile trials during active grasping task . . . . . . . . . . . . . . 62

3.15 Percentage of significant units (factor sensory condition) during active grasping task. . . . . . . . . . . . . . . 63

3.16 ROC score over time for a F5 unit. . . . . . . . . . . . . . . . . . 64

3.17 Distribution of ROC scores for area F5 . . . . . . . . . . . . . . . 65

3.18 ROC score over time for an example unit of area M1. . . . . . . . . 66

3.19 Distribution of ROC scores for area M1 . . . . . . . . . . . . . . . . 66

3.20 ROC score over time for an example unit of area S1. . . . . . . . . . . 67

3.21 Distribution of ROC scores for area S1 during early memory, late memory and grasping. . . . . . . . . . . . . . . . 68

3.22 ROC score over time for an example unit of area AIP. . . . . . . . . . . . 69

3.23 Distribution of ROC scores for area AIP during early memory, late memory and grasping. . . . . . . . . . . . . . . . . . 69

3.24 Decoding models. . . . . . . . . . . . . . . . . . 70

3.25 LDA decoding accuracy per epoch, averaged over five recordings. . . . . . 72

3.26 Decoding accuracy over time for an LDA decoder trained on different objects. . . . . . . . . . . . . . . . . 73

3.27 Decoding accuracy over time for an LDA decoder trained on sensory conditions. . . . . . . . . . . . . . . . . 74

3.28 Decoding accuracy over time for an LDA decoder trained on object identity and sensory conditions. . . . . . . . . . . . . . . . . . 74

3.29 Firing rate of an F5 unit, reacting to the start of tactile stimulation. . . . . 76

3.30 Firing rate of an F5 unit, showing a small activity increase during stimulation. 77 
3.31 Firing rate of an AIP unit, showing no activity changes during the whole task. . . . . . . . . . . . . . . . . . 78

3.32 Firing rate of an AIP unit, reacting to tactile stimulation and plate movement start. . . . . . . . . . . . . . . . . . . 79

3.33 Firing rate of an $\mathrm{M} 1$ unit, reacting to tactile stimulation start and plate movement start. . . . . . . . . . . . . . . . 80

3.34 Firing rate of an $\mathrm{M} 1$ unit, reacting to movement of the plate below the finger. . . . . . . . . . . . . . . . . 81

3.35 Firing rate of an S1 unit, reacting to tactile stimulation start and plate movement start.

3.36 Firing rate of an S1 unit, showing significant differences in activity between conditions.

3.37 Number of significant units at each timepoint for passive stimulation task (factor plate structures). . . . . . . . . . . . . . . . . . . . . 84

3.38 Number of significant units at each time point for passive stimulation task (factor plate movement direction). 


\section{Abbreviations}

AIP Anterior intraparietal cortex

ANOVA Analysis of variance

$\mathrm{BCl}$ Brain-Computer-Interface

ECoG Electrocorticography

EEG Electroencephalography

F5 Ventral premotor cortex area F5

FMA Floating microelectrode array

LDA Linear discriminant analysis

M1 Primary motor cortex

MEG Magnetoencephalography

NSP Neural Signal Processor

PCA Principal component analysis

PRT Positive reinforcement training

PSTH Peristimulus time histogram

ROC Receiver operating characteristic

S1 Primary somatosensory cortex 


\section{Chapter 1}

\section{Introduction}

Our hands are versatile tools that allow us to interact with our surroundings in a variety of ways. Interactions range from grasping things like a cup of tea to determining its temperature, the shape or texture of the cup and social interactions like holding hands with a loved one. This is not only true for us humans but also for non-human primates, who share many characteristics of our hands, most importantly an opposable thumb (Napier 1962). Like humans, monkeys and apes use their hands in a variety of ways. Usage of their hands ranges from grasping tree branches and picking up food to grooming their social partners to form a stronger bond and reduce tension or stress (Dunbar 1991; Terry 1970). Our brains play a critical role in the generation of precise movements, starting with sensory areas that help perceiving our surroundings, to areas that process input and generate relevant outputs, such as movements. While some tasks can be assigned to certain brain areas, such as motor cortex generating movements or visual cortex processing visual input, behaviour is always a result from interactions between brain areas (Jeannerod et al. 1995; Munk 1890; Penfield and Boldrey 1937). For many tasks the exact interactions between brain areas are not yet well studied.

\subsection{Overview over the planning and execution of grasping}

Movements are a defining characteristic of all behaviours. Animals move around to find resources or shelter, move their eyes to explore the world or touch their surroundings to find out more about the world. Due to its high prevalence in all animals, the generation of movements in our brain was one of the first subjects early neuroscientists studied (Fritsch and Hitzig 1870; Lemon 2008; Penfield and Boldrey 1937). One example for an extremely important movement is picking up objects, such as food or nesting material. Grasping is a quite complex behaviour, as it requires integration of inputs from different senses 
which provide information about the external world as well as internal states. To pick up something, an animal first needs to sense where an object is. Object information can come from a wide variety of senses such as vision, smell, echolocation or magnetic senses (Gibson 1958; Griffin et al. 1960; Hasler 1954; Schlegel et al. 2009). Indeed, especially the sense of vision seems to be so useful that it is believed to have evolved at least 40 times independently in the evolution of animals, possibly even up to 65 times, ranging from simple eye spots that only detect the presence of light to more complex eyes that allow to perceive structures and even colours (Fernald 2006; Land and Fernald 1992; Salvini-Plawen and Mayr 1977). In primates, one brain area specifically processes visual information about objects that are about to be grasped: the anterior intraparietal cortex or AIP (Castiello 2005; Gallese et al. 1994; Murata et al. 2000; Sakata et al. 1995; Taira et al. 1990). AIP receives visual information from the visual cortex (figure 1.1). Lesions in AIP lead to problems with fine finger movements in humans and a deficit in grasping (Binkofski et al. 1998).

Additionally to input from external senses, internal senses like proprioception (the sense of ones own body position and self-movements) need to be taken into account in order to determine how the own body is positioned and how posture needs to change in order to successfully reach out and touch the object (Filimon et al. 2009; Grigg 1994). The brain's main task is to make sense of all these different inputs and to plan a first, general movement. If a monkey wants to grasp a fruit that lies in front of it, a reaching movement needs to be initiated, followed by shaping the hand to adjust to the form and texture of the fruit. Hand shape can vary quite a bit, from a very fine precision grip with two fingers (thumb and index finger) to a more powerful whole hand or power grip. Primates are capable of a wide variety of different hand shapes to grasp an even higher number of different objects (Napier 1962; Schaffelhofer et al. 2015; Smeets and Brenner 1999). Planning-related areas like the premotor cortex will start to process a plan for the required grasping movement (Fluet et al. 2010; Hoshi and Tanji 2000; Schaffelhofer and Scherberger 2016). However, the main area responsible for the execution of movements is the primary motor cortex (Fritsch and Hitzig 1870; Kakei et al. 1999; Penfield and Boldrey 1937). Here, sensory input as well as preparatory activity from other areas are integrated and result in motor commands that will eventually lead to movements, such as grasping a fruit and eating it. Once the movement starts, work for the motor cortex is not done. A constant stream of sensory information needs to be evaluated in order to adapt the movement to new events. Once the hand makes contact with the fruit, different mechanosensors on the glabrous (non-hairy) skin of the hand give information about the fruit, such as texture and weight, and proprioceptive and visual signals inform the brain about the location of the object and different body parts (Delhaye et al. 2018; Johnson 2001; Johnson and Hsiao 1992; Saal and Bensmaia 2014). Based on inputs from different senses, which can 
for example help to determine slippage or breaking of the fruit if held to lightly or tightly, the brain determines when the hand shape or applied force needs to be adapted to a more firm or lighter grip (Johansson 1991). As discussed, different brain areas interact in order to produce grasping movement.

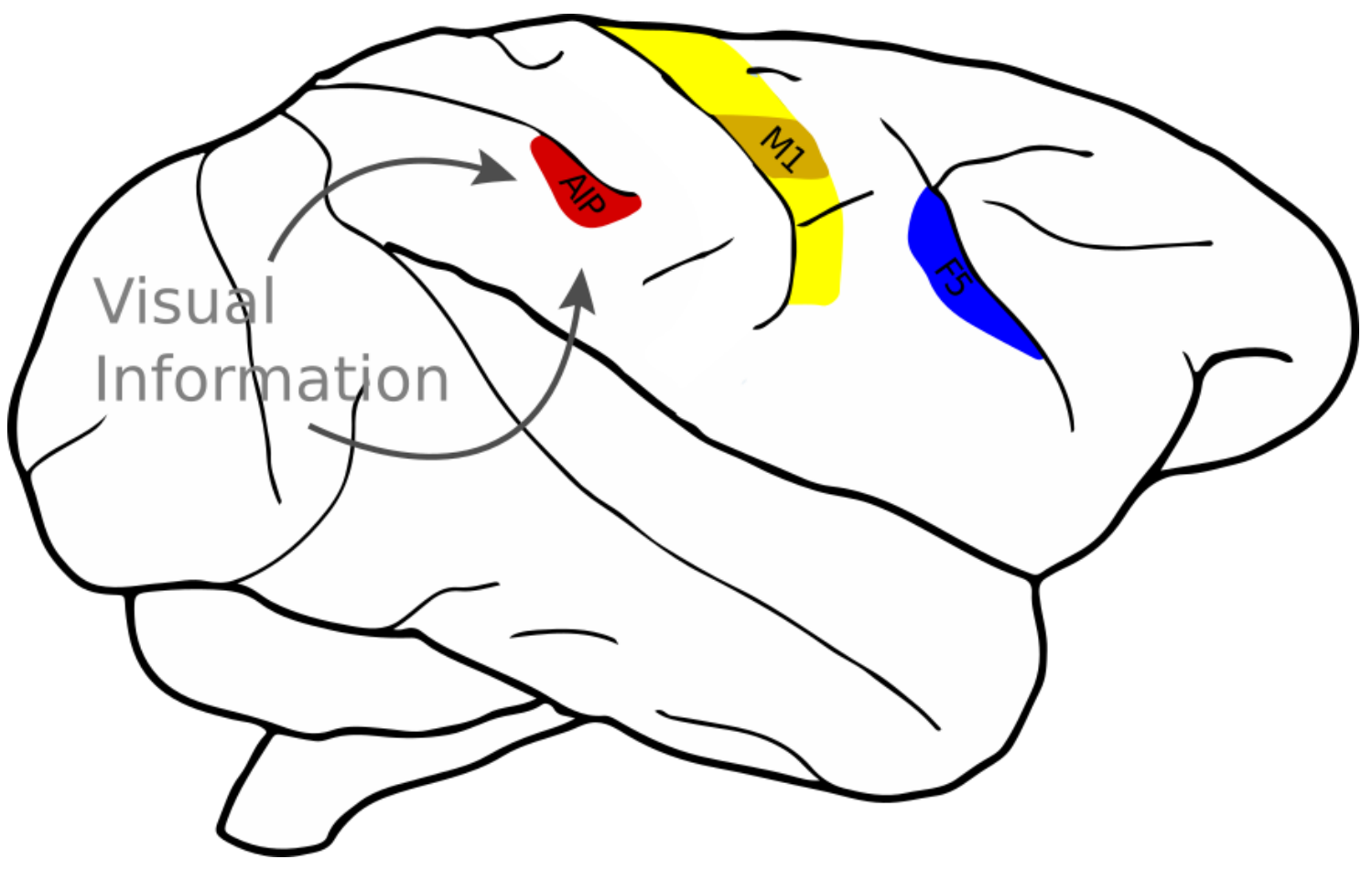

Figure 1.1: The fronto-parietal grasping circuit in the macaque brain. Illustrated is the position of the brain areas AIP, F5 and M1 in the macaque brain (M. mulatta). AIP receives visual information from the visual cortex, while F5 is responsible for movement preparation and M1 for movement execution. The highlighted area in M1 represents hand movements. Illustration adapted from Bowden and Martin 2000.

Three of the formerly mentioned areas (AIP, F5 and M1) form the so called fronto-parietal grasping network and closely interact when objects are grasped (Borra et al. 2007; Menz et al. 2015), see figure 1.1 for their positions in the macaque brain. The primary motor cortex lies at the central sulcus of the brain, while AIP is (as the name implies) located in the anterior parietal cortex. The premotor area F5 sits more to the front.

\subsubsection{Processing of visual information in the anterior intraparietal cortex}

A huge part of the human brain is dedicated to processing visual input from our eyes. Van Essen estimated 2003 that in humans about $27 \%$ of cortex is dedicated to processing predominantly visual input (about $52 \%$ in the macaque brain), while only $7 \%$ each are dedicated to predominantly motor and somatosensory processes (10 and $8 \%$ respectively in the macaque, Van Essen 2003). In the context of grasping movements, as investigated 
in this thesis, one important area to look at is the anterior intraparietal cortex (AIP). AIP is part of the parietal cortex, and is located rostrally in the lateral bank of the intraparietal sulcus (see figure 1.1). It is connected to areas like the secondary somatosensory cortex, premotor area F5 and different ventral visual stream areas (Borra et al. 2007; Luppino et al. 1999). With its connection to the premotor area F5, AIP is part of the fronto-parietal grasping circuit (Begliomini et al. 2014; Binkofski et al. 1999; Luppino et al. 1999). It responds to properties of objects that are about to be grasped and therefore plays a role in object recognition for grasping movements (Borra et al. 2007; Lehmann and Scherberger 2013; Sakata et al. 1995; Taira et al. 1990). In 2009 Baumann et al. recorded AIP neurons during a delayed grasping task, where an animal had to grasp a handle with a precision or power grip (Baumann et al. 2009). The handle could be arranged in multiple orientations, making some grasp planning necessary for the animal since different hand and wrist orientations were needed to successfully grasp the handle. Here, neurons encoded object orientations as well as grip types when the target was known. In a 2015 study, Schaffelhofer et al. trained monkeys to grasp different objects that were visually presented to them (Schaffelhofer et al. 2015). Afterwards, they decode object identity and grasp types from the neuronal data of AIP, both which could be decoded quite well.

The anterior intraparietal cortex therefore builds an important component inside the fronto-parietal grasping network, where it receives and processed visual information of objects and therefore helps to initiate meaningful movements for object interactions.

\subsubsection{The importance of the somatosensory system for grasping}

While the fronto-parietal grasping network covers areas in the primate brain that are needed to grasp visible objects, other senses contribute to grasping actions too. In this project I investigated how tactile perception interacts with the fronto-parietal grasping network but also how the fronto-parietal grasping network processes tactile information.

For most animals, tactile information is essential for survival. One big advantage is that the sense of touch also works in the dark or for objects we can not see. An example for such grasping movements would be reaching for a key in our pockets or trying to find the light switch in the dark. It is also important for animals and humans who are blind and therefore need to rely on their remaining senses to interact with their environment, such as reaching and grasping for objects (Castiello et al. 1993; Stone and Gonzalez 2014). One of the most important brain areas in the primate brain that processes tactile input is the somatosensory cortex (Delhaye et al. 2018; Penfield and Boldrey 1937; Woolsey and Fairman 1946). It sits opposite of the primary motor cortex on the central sulcus (see figure 1.3). Penfield found a somatotopic map, similar to that in $M 1$, where a representation of the body can be found alongside the brain area (figure 1.2). 


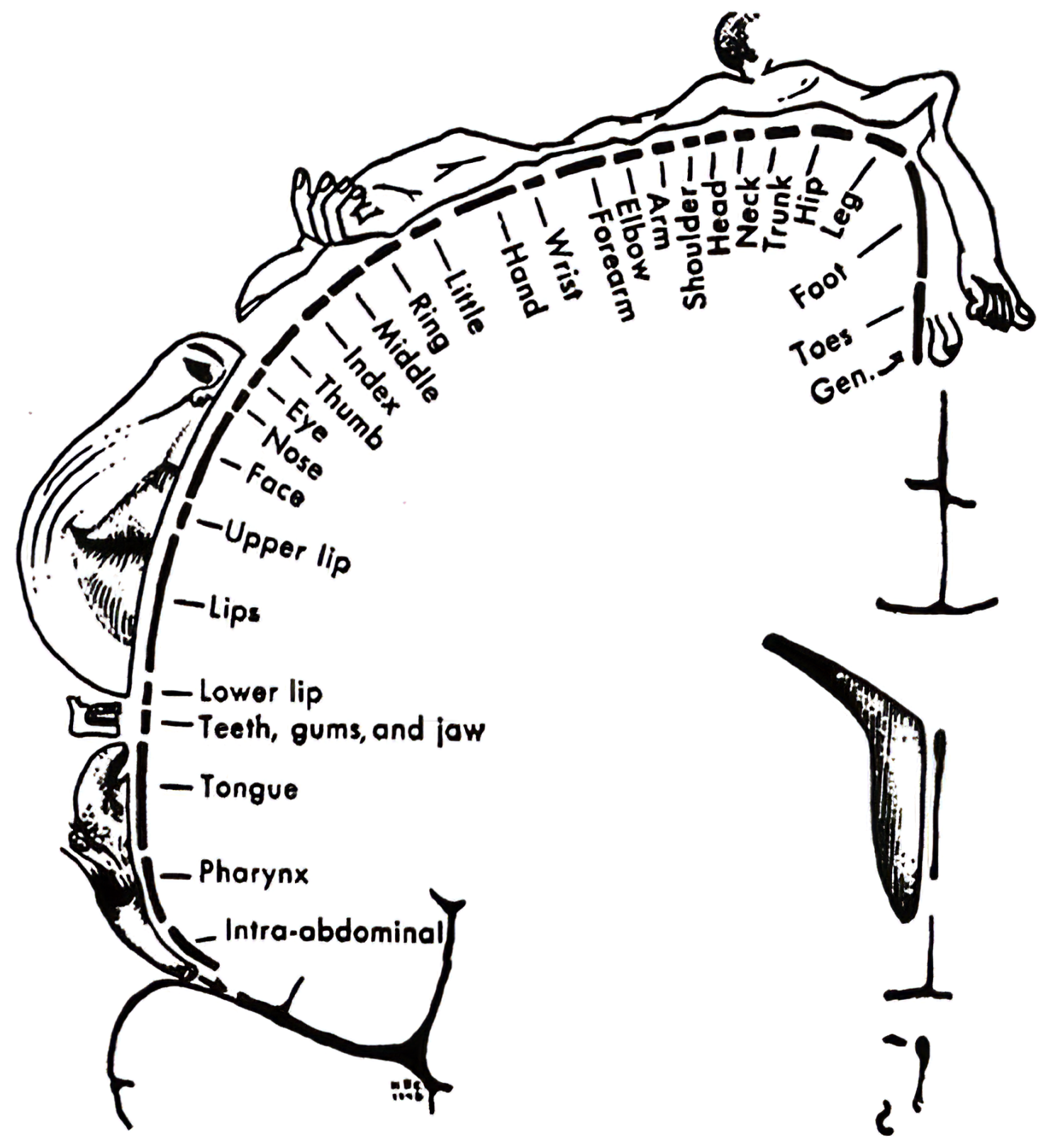

Figure 1.2: The sensory homunculus, representing primary motor cortex. An illustration of neural representation of body parts in the primary somatosensory cortex of humans. The background shows a brain slice, the homunculus drawn on top shows where certain body parts are represented in the brain and how big those areas are (for example, more neurons are responsive to stimulation of the hands, leading to a bigger hand illustration). Adapted from Penfield and Rasmussen 1950.

Body parts that are closer together are also represented by areas that are not far apart from each other, allowing it to draw a sensory homunculus (a "small human") on top of the sulcus. Here, bigger drawn body parts mean that a bigger area of the somatosensory cortex represents these body parts. Over the years, the sensory homunculus was studied by different groups and readapted, for example the position of the genitalia, which seem to be misplaced towards the feet in Penfield's version and are actually closer to the trunk 
according to Rothemund et al. 2002 (in macaques) and Kell et al. 2005 (in humans).

While the primary somatosensory cortex is generally regarded as a single brain area, it actually consists of multiple parts that even respond to different types of sensory information. Overall, the somatosensory cortex responds to a multitude of different sensory inputs, including mechanosensation and theromosensation. It facilitates pain, processes tactile information from our sense of touch, is responsible for proprioception and feeling heat or coldness. The skin itself has different receptors that respond to either one or multiple types of these inputs (Bautista et al. 2007; Peier et al. 2002). Thermoreceptors for example respond to changes in temperatures or certain absolute temperatures (Dubner et al. 1975; Kossen 2019; Vriens et al. 2014).

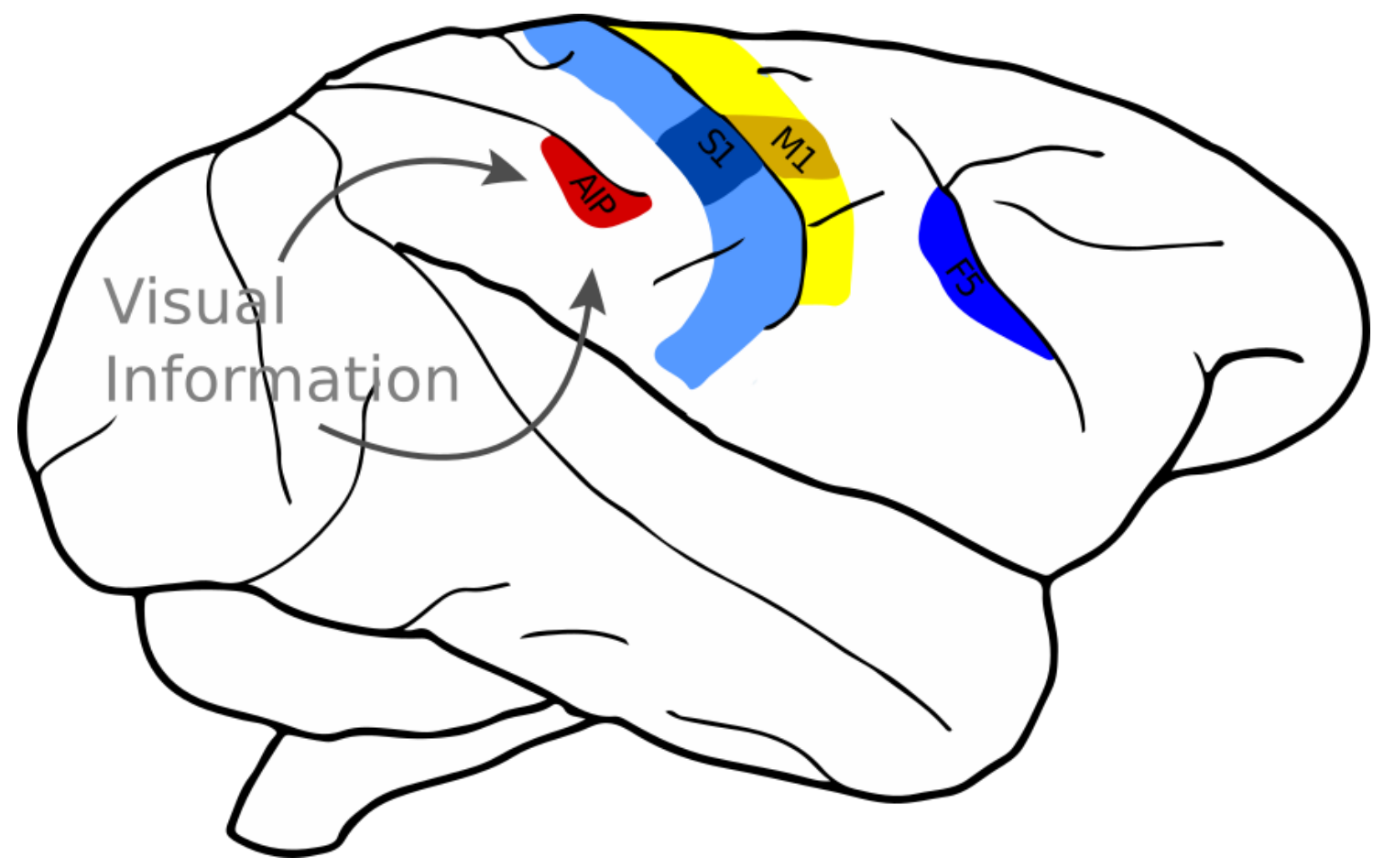

Figure 1.3: The fronto-parietal grasping circuit including $\mathbf{S 1}$ in the macaque brain. IIlustrated is the position of the brain areas AIP, F5, M1 and S1 in the macaque brain ( $\mathrm{M}$. mulatta). AIP receives visual information from the visual cortex, while F5 is responsible for movement preparation and M1 for movement execution. $\mathrm{S} 1$ responds to tactile input. The highlighted areas in M1 and S1 represent the hand areas of both areas. Illustration adapted from Bowden and Martin 2000.

In the early nineteenhundreds, Korbinian Brodmann published his comparative studies of different brain areas, going by cell histology and organization (Brodmann 1909). According to his definition, the primary somatosensory cortex actually consists of three distinct areas, the Brodmann areas 1, 2 and 3. 1919 Cécile and Oskar Vogt concluded that area 3 should be redefined as two areas, called 3a and 3b (Vogt 1919). 


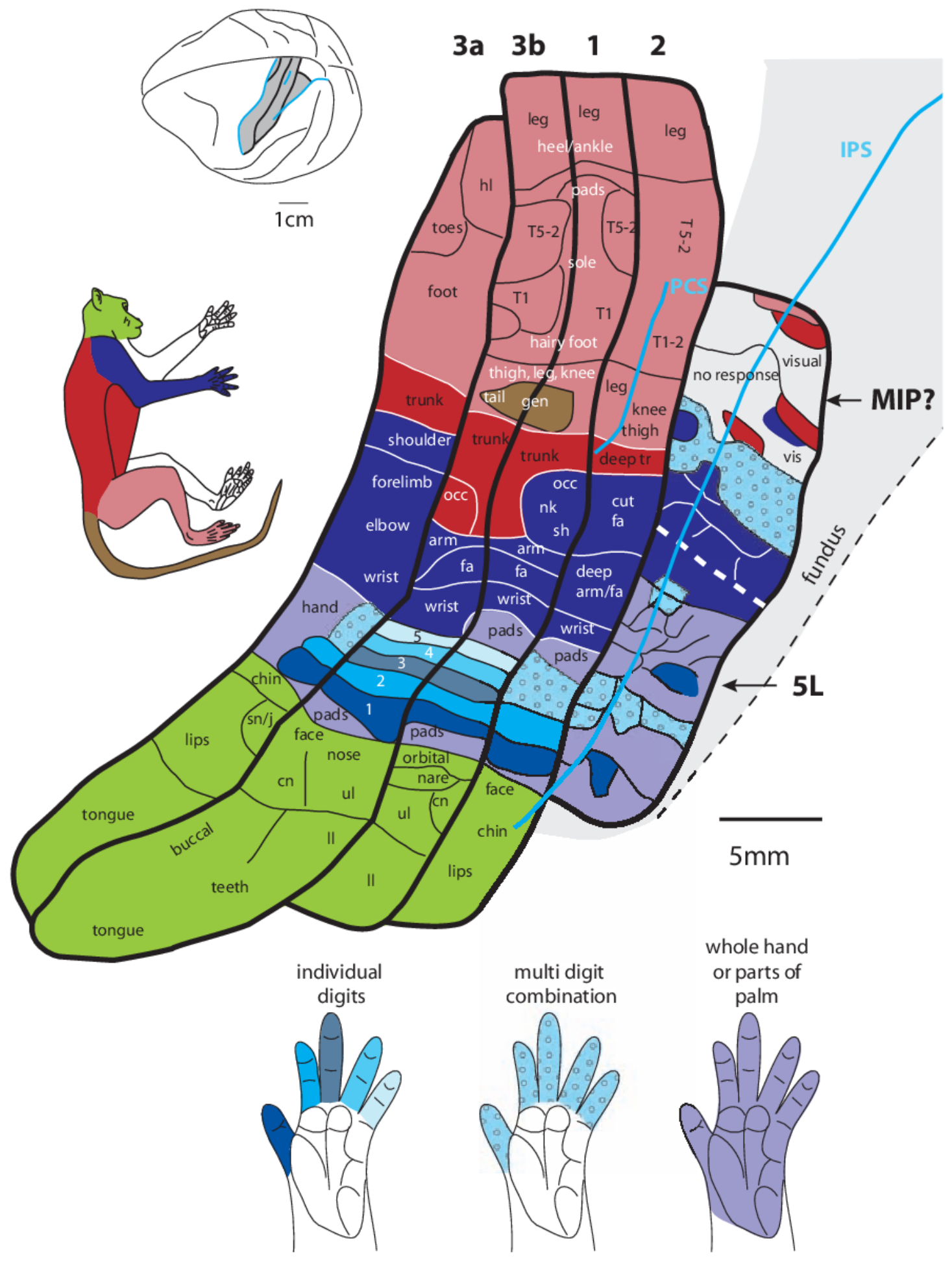

Figure 1.4: Multiple somatotopic representations on Brodmann areas 1, 2, 3a, 3b and 5L. An illustration of the different somatotopic maps alongside the primary somatosensory cortex and the posterior parietal area 5L. While the overall somatotopy is present in all maps, some differences are visible, such as areas 1 and $3 \mathrm{~b}$ encoding individual fingers while some digits are represented together in areas 2 and 3a. Illustration taken from Seelke et al. 2011.

While the sensory homunculus is usually seen as a representation of "the primary so- 
matosensory cortex", more recent studies also concluded that the four different Brodmann areas of $\mathrm{S} 1$ contain their own versions of a sensory homunculus or simiusculus in monkeys (Kaas et al. 1979; Krubitzer and Baldwin 2017; Seelke et al. 2011). Figure 1.4 shows an illustration, taken from Seelke et al. 2011, with different somatotopic maps along the four different areas of S1, based on data from multiple studies (Krubitzer et al. 2004; Nelson et al. 1980; Pons et al. 1985; Rothemund et al. 2002; Seelke et al. 2011).

Brodmann area $3 a$ is mainly responsive to proprioception, while $3 b$ and 1 are more sensitive towards cutaneous sensations like tactile input, although there is no complete separation (Iwamura et al. 1983; Iwamura et al. 1985; Paul et al. 1972). For example, any movements that might be able to elicit proprioceptive responses (as posture changes) usually also leads to deformation of skin and might therefore also activate some types of cutaneous neurons (Bensmaia and Tillery 2014; Delhaye et al. 2018; Edin 1992; Edin 2001; Edin 2004; Hulliger et al. 1979; Kim et al. 2015; Walsh et al. 2014). For grasping, both types of input are important. Proprioception gives information about the current state of the body and therefore is important for future movement plans. Tactile input can provide information once contact has been made, to determine possible adaptations in the hand shape or force. This can be seen especially in humans and monkeys who lost their sense of touch, leading to difficulties with grasping objects due to an inability to determine and maintain an appropriate amount of force (Asanuma and Arissian 1984; Augurelle et al. 2003; Dannenbaum and Dykes 1988; Mott and Sherrington 1985). Due to this importance for hand function, feedback or even an artificial sense of touch is also desired for prostheses, which would increase the usability of artificial limbs. One attempt to deliver feedback to the brain is direct intracortical stimulation of the somatosensory cortex. While animals can not directly report whether or not they feel any sensation when stimulation is delivered to the somatosensory cortex they can be trained to report through behaviour. For example Tabot et al. first delivered mechanical stimuli to the hand of monkeys and trained the animals to report relative locations of the indentation (whether the second poke was lateral or medial of the first, Tabot et al. 2013). Afterwards, some trials were replaced with electrical stimulation, where a prosthetic hand was poked and gave feedback to the animal through electric stimulation in S1. The animal was able to reliable report relative location of the electric stimulation, showing that the electric stimulation elicited signals that the animal could use to continue reporting stimulation locations. Furthermore, different groups were able to use intracortical stimulation in the somatosensory cortex of human patients, to deliver feedback to the participants (Flesher et al. 2016; Salas et al. 2018). There, the patients reported different sensations, ranging from electrical, artificial sensations to more natural ones like tingling or tugging on the arm. 


\subsubsection{The premotor cortex and the planning of movements}

Just as the name implies, the premotor cortex is known to be involved in movement planing (Churchland et al. 2006). It is located in Brodmann area 6, where later studies found different distinct areas, named F2 to F7, based on cyto- and myeloarchitecture (Barbas and Pandya 1987; Brodmann 1909; Rizzolatti and Luppino 2001; Rizzolatti et al. 1998). Barbas and Pandya 1987 hypothesised that these distinct areas might hint towards different specialisations based on the very widespread connections the premotor cortex has to other areas. In later studies, area F2 was investigated as a grasping- or reaching related area, showing neurons that either responded to planning of movements or to visual stimuli like the presentation of objects (Churchland et al. 2006; Cisek and Kalaska 2005; Raos et al. 2004). Fogassi et al. 1999 and Raos et al. 2003 also showed that most body parts (excluding the face) are represented in it, although most visually sensitive neurons seem to be closer to the area that also represents forelimb movements. Area F4 on the other hand, is known to be a more sensory area that responds to different sensory inputs that also controls face and arm movements (Fogassi et al. 1996; Rizzolatti et al. 1988; Rizzolatti et al. 2002; Rizzolatti et al. 1981). Most relevant for this project is area F5, which reacts to movement execution and observation (Hepp-Reymond et al. 1994; Rizzolatti et al. 1996; Weinrich et al. 1984). Kurata and Tanji 1986 recorded single neuron activity from neurons in the premotor cortex of monkeys who were trained to do hand and arm movements. There, activity before movement started could be found in hand and arm movements. Later Murata et al. 1997 could show that these activity changes were not only related to arm and hand movements but also to the presentation of 3D objects. The hand area of the premotor cortex, F5, is directly and bi-directionally connected to AIP, and both areas are part of the fronto-parietal grasping network (Borra et al. 2007; Luppino et al. 1999; Rizzolatti and Luppino 2001).

\subsubsection{Generation of movements in the primary motor cortex}

The primary motor cortex (M1) is one of the most important areas for movement generation. M1 lies just anterior to the central sulcus and stretches from the top of the cortex almost all the way down to the lateral fissure (see figure 1.1). Early studies were already able to detect a relationship between electric stimulation of $\mathrm{M} 1$ and movements of the body, where twitches would be elicited with low-current electrical stimulation electrodes (Clark and Ward 1948; Cooper and Denny-Brown 1927). Interestingly, a representation of the body can be found in M1 (see figure 1.5, as defined by Penfield and Rasmussen 1950), where stimulation of certain parts of $\mathrm{M} 1$ leads to reliable and reproducible twitches in certain body parts. 


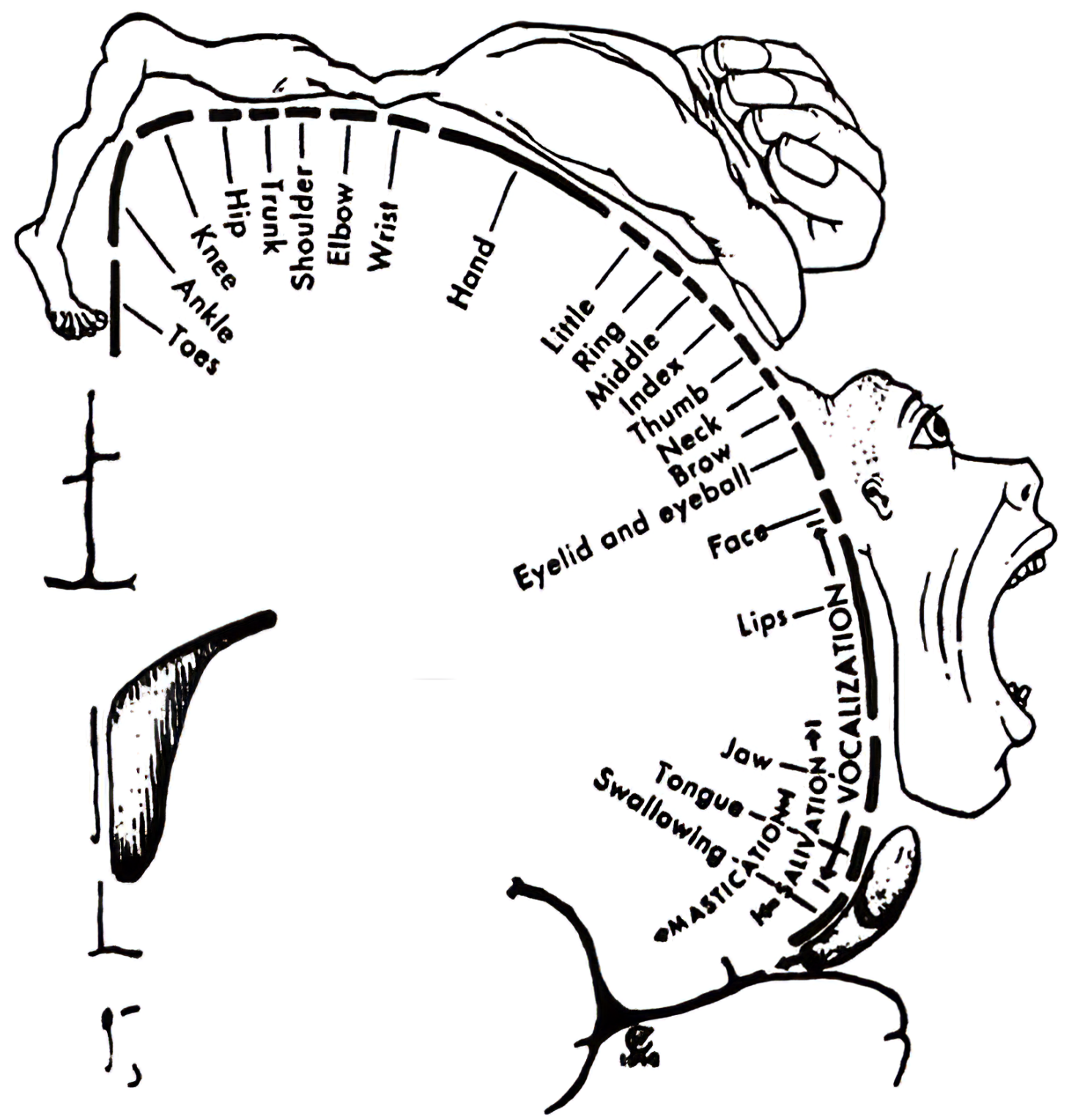

Figure 1.5: The motor homunculus, representing the primary motor cortex. An illustration of the neural representation of body parts in the primary motor cortex of humans. The background shows a brain slice, the homunculus drawn on top shows where individual body parts are represented in the brain and how big those areas are (for example, more neurons are responsible for hand movements, leading to a bigger hand illustration). Adapted from Penfield and Rasmussen 1950.

Similar to the sensory homunculus of the somatosensory cortex (see section 1.1.2) body parts that are close to each other (for example hands and arms) are also represented by nearby parts of the motor cortex, allowing one to draw a theoretical homunculus on top of the cortex. Similar to the sensory homunculus, where body parts that are more sensitive are represented by more cortex area, more dexterous body parts usually occupy a bigger part of the motor cortex. Penfield and Rasmussen chose to represent this relationship by drawing the corresponding body parts bigger, leading to a seemingly distorted 
image which illustrates the relative sizes and location of all body part representations in the motor cortex. At the level of fingers, however, this somatotopic motor representation breaks down and starts to get more mixed (Schieber and Hibbard 1993).

While it is generally accepted that some kind of somatotopic organisation exists, it is less clear what kind of parameters M1 neurons actually represent, such as force, movement direction or muscle activation (Evarts 1968; Georgopoulos et al. 1982; Mussa-Ivaldi 1988; Sergio et al. 2005; Todorov 2000). While Penfield and Boldrey 1937 were able to elicit small muscle twitches, Graziano et al. 2002 were able to elicit entire limb movements by stimulating motor cortex for a longer duration ( $500 \mathrm{~ms})$. These movements usually consisted of complex movements(see figure 1.6), such as leading a hand towards the mouth, with the final posture being independent of the starting point of the hand.

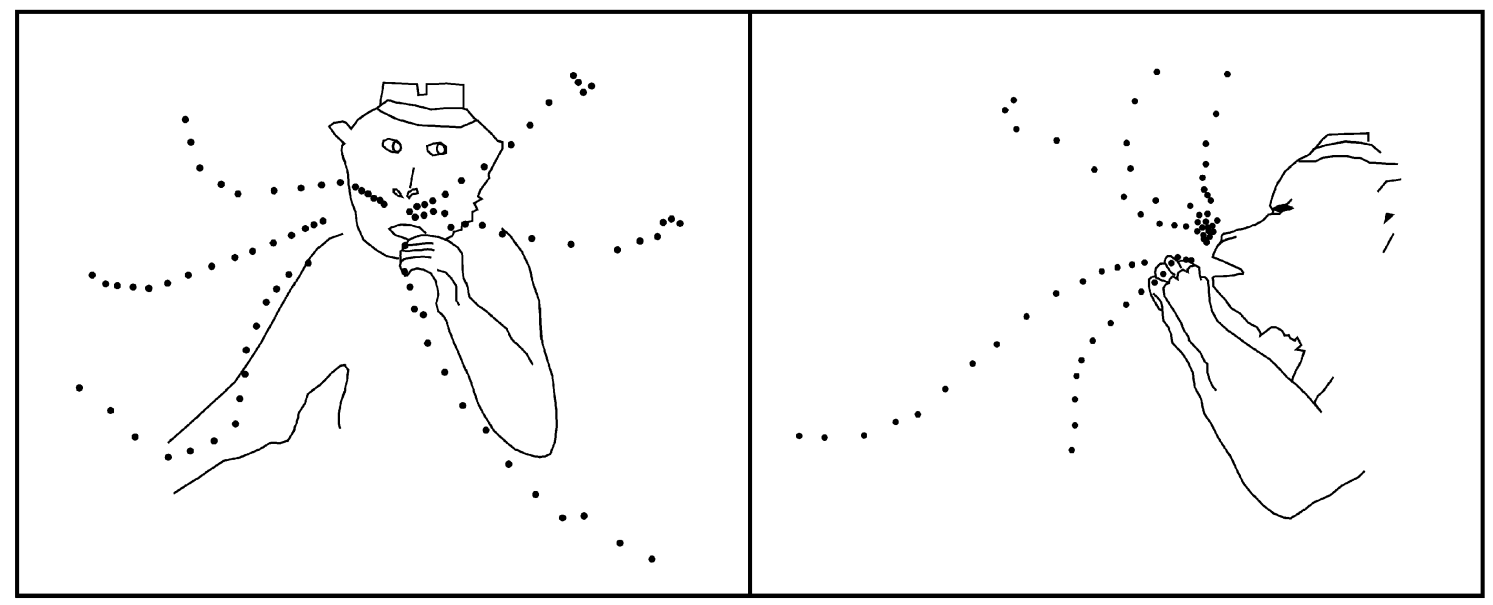

Figure 1.6: An example movement elicited by stimulation of primary motor cortex. One example movement elicited by stimulation (duration 500ms) of M1. Independent of the starting point, stimulation of M1 led to a movement that ended at the mouth of the animal. Illustration taken from Graziano et al. 2002.

Still, even though there is an ongoing discussion on what M1 actually represents, its activity has been successfully used for prosthetic control of robotic arms (Adewole et al. 2016; Collinger et al. 2013; Velliste et al. 2008). Chapin et al. were able to decode neuronal activity recorded from rats (Rattus sp.) and trained the animals to position the arm to receive rewards (Chapin et al. 1999). Wessberg et al. were able to reproduce these findings in owl monkeys (Aotus trivirgatus, Humboldt, 1811), where the animals were trained to execute one-dimensional hand movements and three-dimensional reach movements for food, based on neuronal recordings (Wessberg et al. 2000). First implants in human patients were capable of similar feats, where quadriplegic patients can control computer cursors or robotic arms with brain recordings (Hochberg et al. 2006; Simeral et al. 2011; Truccolo et al. 2008; Wodlinger et al. 2014), demonstrating that although many questions about the primary motor cortex are still open, successful control of robotic limps is actually possible, with some limitations. 


\subsection{Introduction to neural interfaces}

Today, many methods exist to record the neural activity of the brain, which offer different up- and downsides. An overview of recording methods, taken from Astrand et al. 2014 is illustrated in figure 1.7. A comparison of spatial and temporal resolutions of different methods, taken from Van Gerven et al. 2009, can be seen in figure 1.8.

When recording brain activity, different advantages, risks and limitations need to be considered.

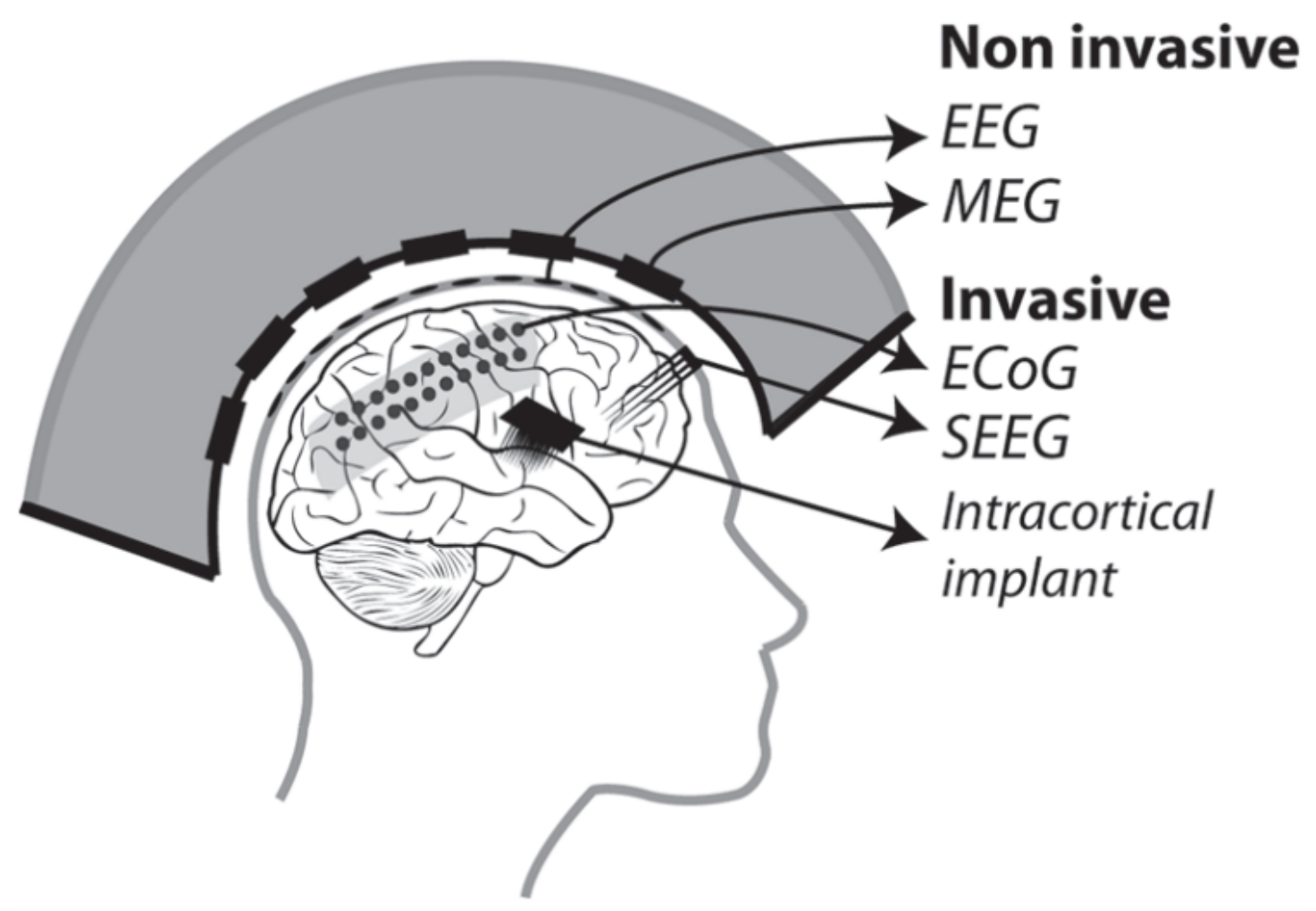

Figure 1.7: Illustration of different methods to record brain activity. An illustration of different methods to record brain activity, illustrating where they are placed. Shown are electroencephalography (EEG, on the scalp), magnetoencephalography (MEG, around the head), electrocorticography (ECOG, on top of the dura), stereoelectroencephalography (SEEG, inside cortex, through the skull) and intracortical implants (implanted into cortex). Illustration taken from Astrand et al. 2014.

One method that is non-invasive (meaning it requires no surgery) is the electroencephalography (EEG), where electrodes are placed on top of the scalp. Here brain activity can still be picked up, although filtered through the skull, which acts as a spatial low-pass filter and therefore decreases signal strength and with low spacial resolution in the range of centimetres (Berger 1929; Buzsáki et al. 2012; Im and Seo 2016; Srinivasan 1999). While some of the signal is lost, it still helps to roughly localise the origin of signal and offers a good temporal resolution, allowing for the control of cursors or small helicopters in up 
to three dimensions or the steering of wheelchairs (McFarland et al. 2010; Tanaka et al. 2005). With Magnetoencephalography (MEG) a similar spacial and temporal accuracy to that of EEG recordings can be reached (Sejnowski et al. 2014; Van Gerven et al. 2009). For MEG recordings, no electrodes are placed on the participants, instead a magnetic SQUID (superconducting quantum unit interference devices) is placed around the head and is used to measure changes in electro-magnetic activity (Cohen 1968; Hall et al. 2014). This method is less mobile than EEG caps but also applicable without surgeries. While the signal is again dampened by the skull, the signal quality allows for applications like neurofeedback for opening and closing of a virtual hands (Foldes et al. 2015).

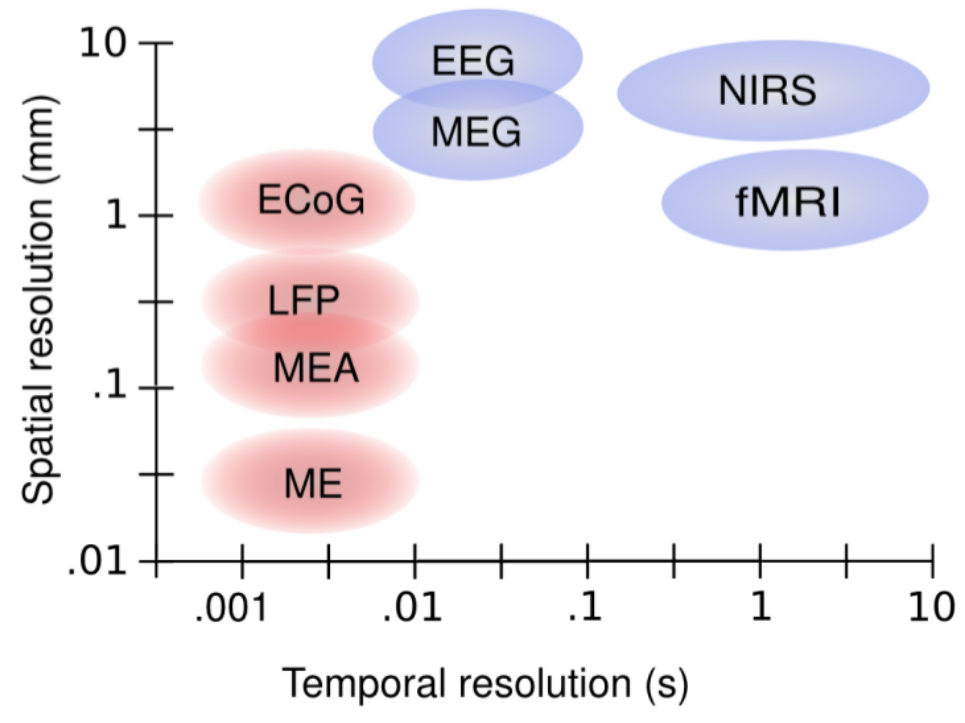

Figure 1.8: Comparison of spacial and temporal resolution of different recording methods. This illustration compares the spatial and temporal resolution of different recording methods. While Near-infrared spectroscopy (NIRS) and functional magnetic resonance imaging (fMRI) have a low spatial and temporal resolution, electroencephalography (EEG) and magnetoencephalography (MEG) have a low spatial but better temporal resolution. Electrocorticography (ECoG), local field potential (LFP), multi-electrode arrays (MEA) and microelectrode (ME) have the best temporal resolution but a varying spatial one. Illustration taken from Van Gerven et al. 2009.

A better spatial resolution can be achieved using electrocorticography (ECoG, sometimes also called intracranial electroencephalography), where an electrode grid is placed on the surface of the brain. In contrast to MEG and EEG, ECoG is an invasive method (sometimes also called semi-invasive, since the skull is opened but nothing is implanted into the cortex) and allows decoding of different movements due to its higher temporal resolution (Flamary and Rakotomamonjy 2012; Pistohl et al. 2008). Still, since the signal is recorded on top of the cortex, some spatial resolution is lost. Single micro-electrode (ME) recordings, which are usually not chronically as well as Micro-electrode arrays (MEAs), where multiple electrodes are implanted into the cortex, provide an even better spatial resolution (Hubel and Wiesel 1959; Im and Seo 2016; Marg and Adams 1967; Musallam et 
al. 2007). Both methods are invasive, since they require a surgery. For single electrode recordings, the skull is opened and the electrode is driven into cortex through the dura, MEAs are usually chronically implanted (see section 2.2 for an example). While these methods offer a very good temporal and spatial resolution, the surgery poses some risks (Van Gerven et al. 2009). First, typical surgery risks come to mind. The patient needs to be placed under anaesthesia, skin and skull are opened and therefore vulnerable to infections or other complications (Arbous et al. 2001; Haridas and Malangoni 2008). While sterile conditions make these complications rare, they are not present in non-invasive methods, giving those a big advantage at the cost of the lower temporal and spatial resolution. After electrodes are implanted, one usually has to go with whatever result was achieved, since all possible corrections would require a second surgery and further complications like scarring of the dura or around the implants might be present (Barrese et al. 2013; Edell et al. 1992; Harris and Tyler 2013). Furthermore, while implanted electrodes can be used for months to years in animals and patients, they do not last a lifetime (Chestek et al. 2011; Nicolelis et al. 2003). Reasons for later failure of a successfully implanted electrode can be of biological (such as bleeding or scarring) or mechanical (breakage of cables or displacement) (Barrese et al. 2013; Polikov et al. 2005; Schmidt et al. 1993; Stensaas and Stensaas 1978; Turner et al. 1999). Still, the better temporal and spatial resolutions allow for a better understanding of activity patterns on a nearly neuronal level, since electrodes are capable of picking up signals from single or multiple neurons around their tip, while non-invasive methods usually only allow a rough localisation of an area but not individual neurons (Gray et al. 1995; McNaughton et al. 1983). This is especially important if one wants to look at fine movements such as grasping, where the movements of individual fingers are relevant and a high temporal resolution is required to keep up with the fast movements.

\subsection{Motivation and objectives of this thesis}

In order to grasp objects, we need to find an appropriate strategy to do so, which includes estimating the best hand shape as well as regulating the executed force (Feix et al. 2014a; Feix et al. 2014b; Napier 1956; Wheat et al. 2004). Usually we are able to preshape our hand even before we actually touch or lift an object, demonstrating how well we can evaluate an object and estimate the best grip by using information from different senses. Just by looking at a cube, we already get a lot of information about it, such as size and texture and we are able to plan a grasp that will work for this particular cube. Another way to acquire size and texture information would be by touching the cube beforehand and feeling the surface to get information about texture and size. Acquiring tactile information works even in the dark, in absence of any visual cues. In both cases, we are 
informed enough to generate a hand shape that will make the cube fit in our hands quite well, with only small adjustments necessary once we actually start lifting the object and can also account for weight and slip.

From a neurobiological standpoint that means that our brain needs to process very different information in a way that leads to a similar outcome. For the brain it does not matter whether we saw the cube with our eyes and processed the object in visual cortex before planning started or whether we touched it with our fingers and processed the information through the peripheral nervous system and somatosensory cortex. The exact hand shape might differ, but the overall outcome is the same: We can readily lift the object.

The goal of this project was to find out how the brain manages to do this task and whether information acquired by our eyes or hands are handled differently or similarly in F5 and M1. Overall goal of this thesis is the comparison of representations of tactile and visual information in the context of planning a grasp in the brain.

In a first experiment, I wanted to know how different textures are represented in areas which are part of the fronto-parietal grasping network (AIP, F5, M1 and S1), when these textures are presented passively (section 2.6). Five different grating sizes were chosen that were presented to the middle finger of the monkey, either from right to left or from left to right. In this experiment I could find strong significant differences in areas S1 and weaker but still significant differences in $\mathrm{M1}$, during finger stimulation. In the areas F5 and AIP, no significant differences were found, indicating that these areas are less sensitive to tactile inputs in the absence of grasping movements (section 3.4).

In a second experiment I directly compared how visual and tactile information is processed in the context of grasping movements in areas AIP, F5, M1 and S1 (section 2.5). Here, I decided to use a setup where I can present different objects to the animal and instruct him to either explore them tactually in the dark or to just look at them, before the monkeys needs to lift the objects up (section 2.5.1). Focusing on the memory period, where the animal memorises the object and plans his grasping movement, I could show a significant difference in most brain areas, indicating that grasps are planned differently when based on visual or tactile information (section 4.1). The differences are even big enough to allow decoding of the sensory condition during memory period. During grasping, differences become less pronounced although still big enough for an LDA decoder to differentiate between both conditions.

Together both experiments could demonstrate how tactile information is processed in the fronto-parietal grasping network and the primary somatosensory cortex. During grasping (and tactile exploration) the motor and tactually driven areas F5, M1 and S1 showed strong tuning during movements, which were absent in F5 during passive stimulation and only weakly present in M1 during the same passive finger stimulation experiment. AIP 
responded mainly to visual information and only secondary to movements in the active grasping experiment and showed no tuning during the passive finger stimulation, most likely due to the absence of visual information and grasp intentions. These results have some implications when one tries to use information from the four brain areas investigated in this thesis in order to control a $\mathrm{BCl}$-driven prosthesis. While during movement execution only small differences in neuronal activity were present, these differences were strong enough during grasping and during memory period to allow above chance level decoding of the sensory condition. This means when one tries to decode a grasp movement, taking the source of the object information (such as vision or the sense of touch) into account will refine decoding and might therefore allow a better control. 


\section{Chapter 2}

\section{Materials and Methods}

In this chapter, the materials and methods used for this project are described in detail. In section 2.1 the animals (monkey Homer and monkey Ralph) are introduced. An overview of the implantation technique and neural recordings is given in sections 2.2 and 2.3. The following few sections give an overview of the training of the animals (section 2.4) as well as the two different experiments the animals were trained on (sections 2.5 and 2.6). The last sets of sections cover the different ways the data was analysed section 2.7 .

\subsection{Subjects}

Two monkeys were trained for this study, Monkey Ralph (born March 2010) and Monkey Homer (born May 2009). Both animals are male, purpose-bred rhesus macaques (M. mulatta, Zimmermann 1780, see figure 2.1), who were born at the German Primate Center (Deutsches Primatenzentrum GmbH, Göttingen, Germany) and housed together in the animal facility of the Neurobiology Laboratory with a 12 hours dark-light cycle (12h light, 12h darkness).

The animals had access to different types of regularly changed enrichment, such as swings, tree trunks, puzzles and other toys, and could observe other monkeys, males and females, inside the facility, which live in adjacent rooms. Wood chips (German Horse SPAN Premium, German Horse Pellets Vertrieb GmbH \& Co. KG, Wismar, Germany) on the floor encourage foraging. Occasionally, small treats like sunflower seeds, different types of nuts and raisins were either placed into the puzzles or hidden in the wood chips to encourage interaction with the toys and puzzles. During training and recording, animals had free access to food such as pellets with a high nutrition value ('Old World Monkey Chunks" by Special Diet Services Deutschland, Ludwighafen, Germany and "6029 - Extrudate" by Altromin Spezialfutter GmbH \& Co. KG, Lage, Germany) and occasionally nuts, seeds, and 

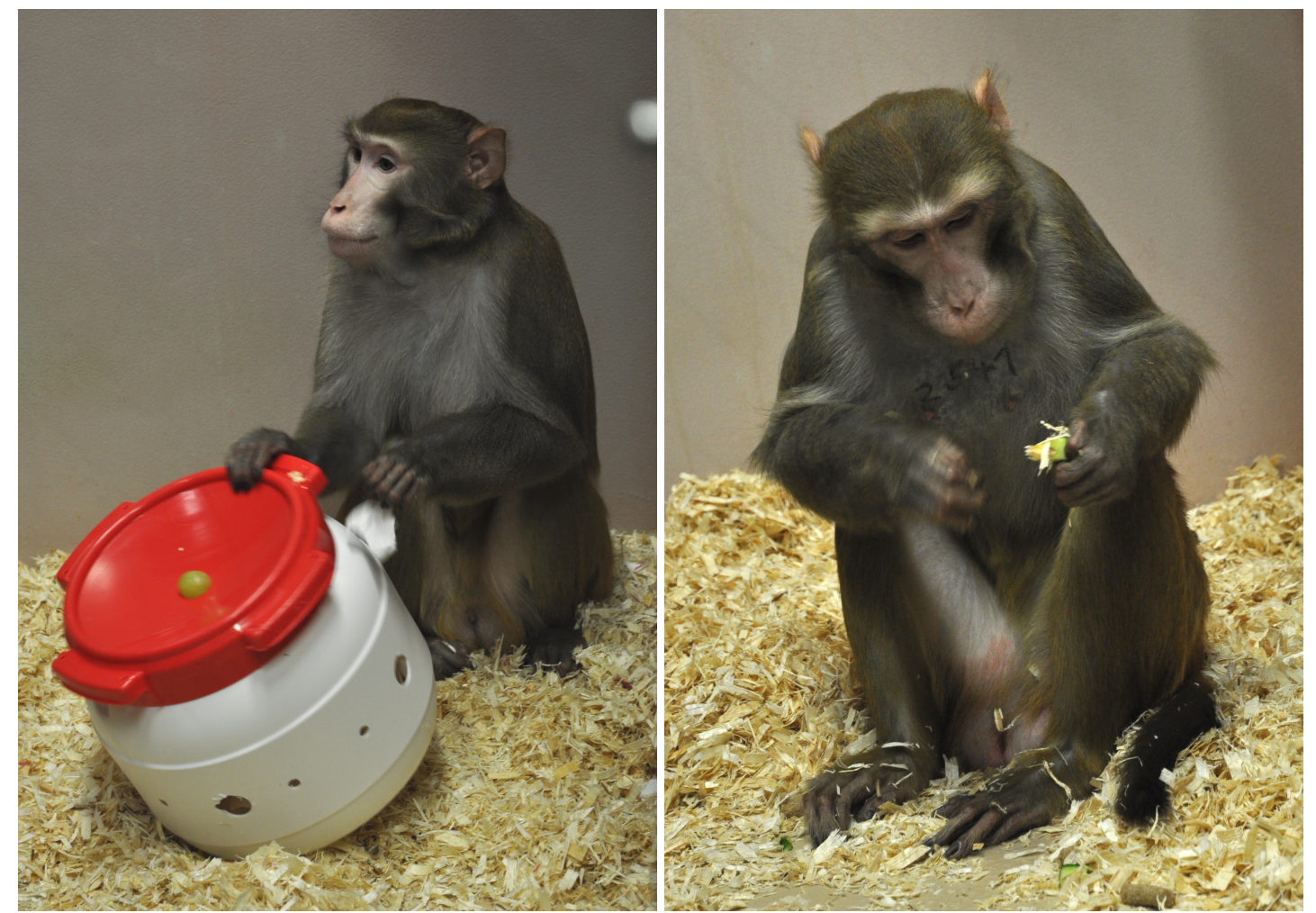

Figure 2.1: Images of monkeys. Images of the two macaques (M. mulatta) trained for this project. Left: Monkey Ralph, Right: Monkey Homer

different dried fruits. Intake of fluids, in form of water and juice or fruits and vegetables, was monitored and fluids were used as main reward. The weight of the animals was controlled regularly before each training or recording session and regular health checks were performed. On days where no training took place, the monkeys had free access to water or juice, pellets, fruits and vegetables. This is also true for training days, if the following day would be free: In these cases free access was granted after training or recording was finished.

Housing and all experiments were performed in accordance with European and German law (EU directive 2010/63/EU; TierSchG; TierSchVersV) and in agreement with the "Guidelines for the Care and Use of Mammals in Neuroscience and Behavioural Research" (National Research Council 2003) as well as with the NC3Rs "Guidelines for Non-human primate accommodation, care and use" (National Centre for the Replacement, Refinement and Reduction of Animals in Research 2017).

\subsection{Implantation}

The surgical procedures used in this project have been described in detail before (e.g. Michaels et al. 2015), therefore only an overview is given here. After the animal reached 
a consistent task performance (task performance error per session less than $10 \%$ ) an anatomical MRI scan was performed to locate specific anatomical landmarks, such as the central or arcuate sulcus. This allowed to plan placement of implants before surgery. In a first surgery, a custom made titanium head post was implanted onto the skull of the animal and secured with bone cement (Palacos MV+G by Heraeus, Hanau, Germany). After full recovery (1-2 weeks) the animal learned to accept being head fixed and afterwards returned to task training, now under head-fixation. The second surgery, implantation of floating microelectrode arrays (FMA, by Microprobes for Life Sciences, Gaithersburg, MD, USA, see Musallam et al. 2007) was performed after the animal got used to working while its head is fixed and once again reached a task performance of over $90 \%$.

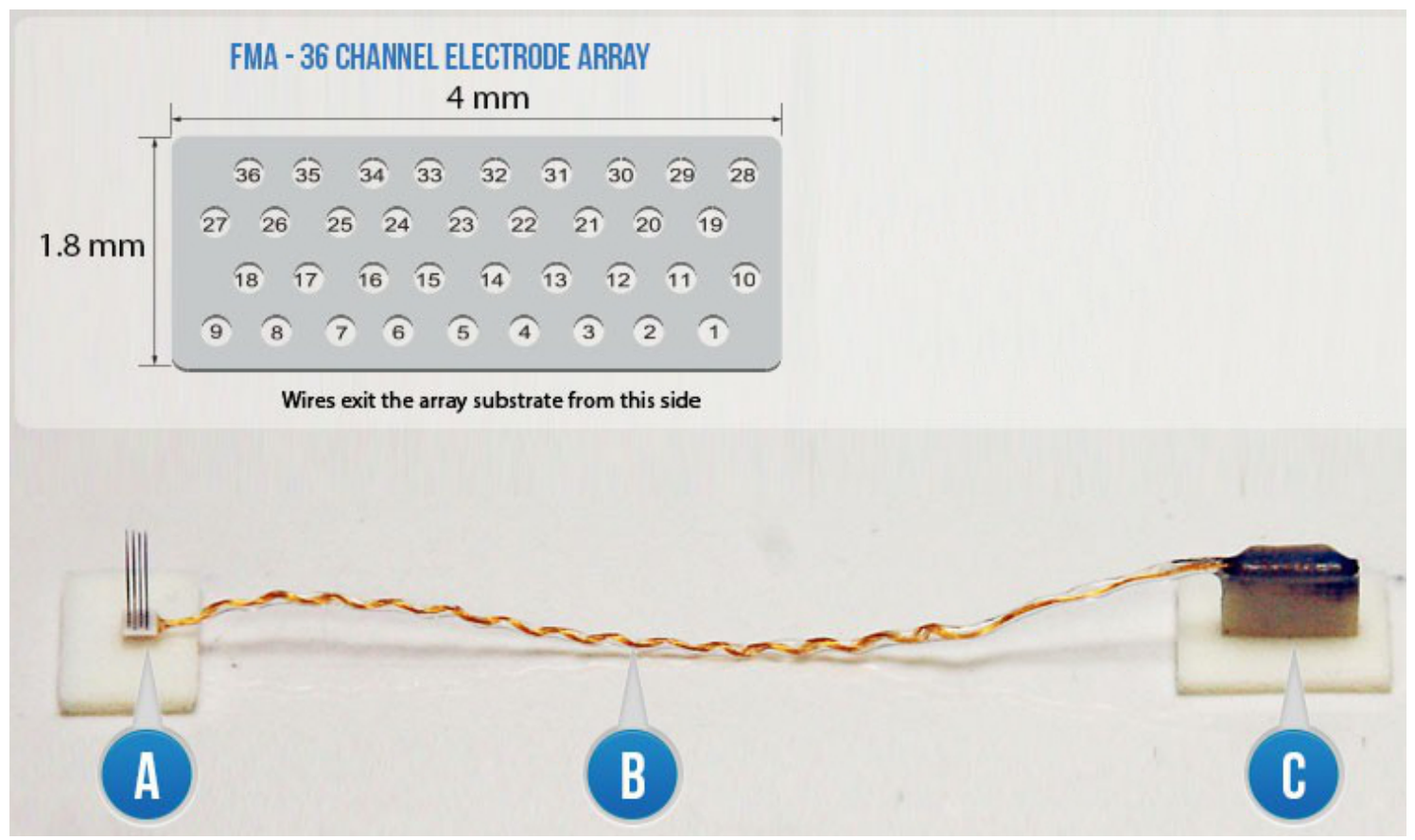

Figure 2.2: Picture of a floating microeletrode array. Images of an FMA including gold cable and connector. The array fits 36 electrodes of varying length $(1.5-7.1 \mathrm{~mm})$ and is $2 \times 4 \mathrm{~mm}$ in size. Spacing between electrodes: $400 \mu \mathrm{m}$.. (Top) Example layout of an array. Electrodes 1, 18, 19 and 36 are used as ground and reference electrodes, the remaining 32 electrodes can be used for recording. (A) The array with the electrodes, (B) the thin gold cable, (C) The connector used to connect the array with the recording hardware. The array is placed on the brain, with the electrodes protruding into cortex, the connector stays outside of the skull. (Picture adapted from https://microprobes.com)

During the second surgery, eight FMAs were implanted into four brain areas: M1, S1, F5 and AIP (two per area). Since in this study I was mainly interested in tactile input, implantation was aimed at area $3 \mathrm{~b}$ of the somatosensory cortex, which is sensitive to cutaneous stimuli (as described in section 1.1.2). For the other areas the known locations of hand representations were implanted, which can be determined by looking at land marks of the brain. Each FMA consists of 36 electrodes of varying length (1.5 - 7.1mm). Two of the electrodes serve as reference, two as ground and the remaining 32 electrodes 
can be used to record extracellular potentials (spiking activity and local field potentials). Two FMAs were inserted per area to increase the number of electrodes per brain area (2x32 or 64 electrodes).

The longest electrodes were placed closest to the respective sulcus, ensuring that all electrodes are embedded in cortex. To ensure this, two variations of the arrays were ordered, differing only on which side the cable exits. Due to the curvature of the sulci, the exact depth of the penetration is uncertain. Additionally, since the arrays are floating on top of the cortex and are not fixed, they might be pushed in or out of the cortex after implantation, which again makes the exact depth of each electrode unknown.

For the implantation of the FMAs a craniotomy needs to be performed (figure 2.3). After the bone cement is removed above sensorimotor, premotor and parietal cortex, a minimal craniotomy is performed. Using the aforementioned MRI scans, size and location were optimized, so that all desired brain areas can be accessed. After opening a small window in the skull, the removed bone piece is stored in sterile $\mathrm{NaCl}$ during surgery and later placed back. Next, the dura mater is carefully cut open. During the whole surgery the brain and dura are kept moist in order to prevent damage to these delicate structures. After the brain is exposed, the FMAs are inserted slowly at a speed of roughly $1 \mathrm{~mm} / \mathrm{s}$ to allow cortex to adjust to the tiny electrodes penetrating it. After all FMAs are placed, the dura is folded back onto the hole, secured with a few stitches and a protein matrix is placed on top, to help with healing (Duragen Plus by Integra LifeSciences Corporation, Plainsboro, NJ, USA). The removed piece of bone is placed on top of the craniotomy hole and surrounded by bone replacement material (chronOS Putty by Mathys AG Bettlach, Switzerland) to facilitate bone regrowth. In a last step the bone cement is closed again to seal everything off. The thin gold cables of the FMAs fit through the gap between skull and bone piece that was created by the cranial drill. Connector and cables are embedded and secured in the bone cement on top of the skull to allow access. The end of the implants features a male omnetics connector (Omnetics Connector Corp., USA) with 36 pins (one pin per electrode) and four guide posts. This connector is embedded in a small titanium pedestal that is placed on top of the bone cement. Outside of recording sessions, this titanium pedestal is covered with a small lid that is held in place with two screws to protect the connector against dirt and mechanical damage. 

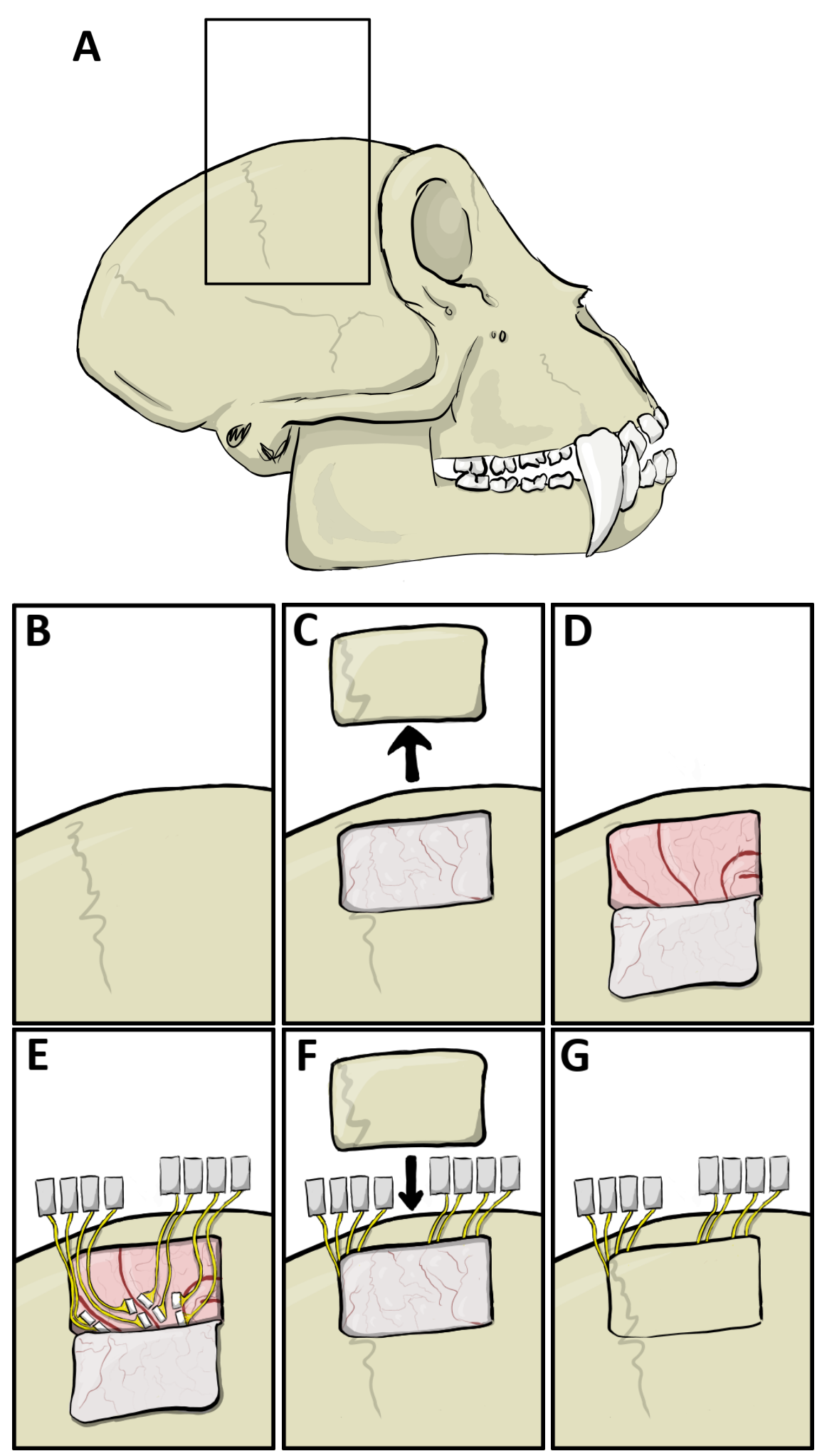

Figure 2.3: Overview of the implantation process. A short overview of the implantation process for FMAs. (A-C) A craniotomy was performed above the sensorimotor, premotor and parietal cortex to allow access to M1, S1, F5 and AIP. (D) Afterwards the dura mater was opened. (E) Eight FMA arrays were inserted into the four brain areas, two per area. (F-G) Afterwards, the dura is closed and the removed bone piece is placed back into place. The cables of the FMA arrays are small enough to fit through the small gap between the bone piece and skull. 
Monkey R. was implanted with the custom made titanium head post in October 2017. Eight microelectrode arrays (FMAs for Life Sciences) were implanted in four different brain areas (see section 2.2) in December 2017. All data for the projects were recorded from Monkey R. between January 2018 and December 2019 and the best sessions were chosen afterwards (see section 3.1).
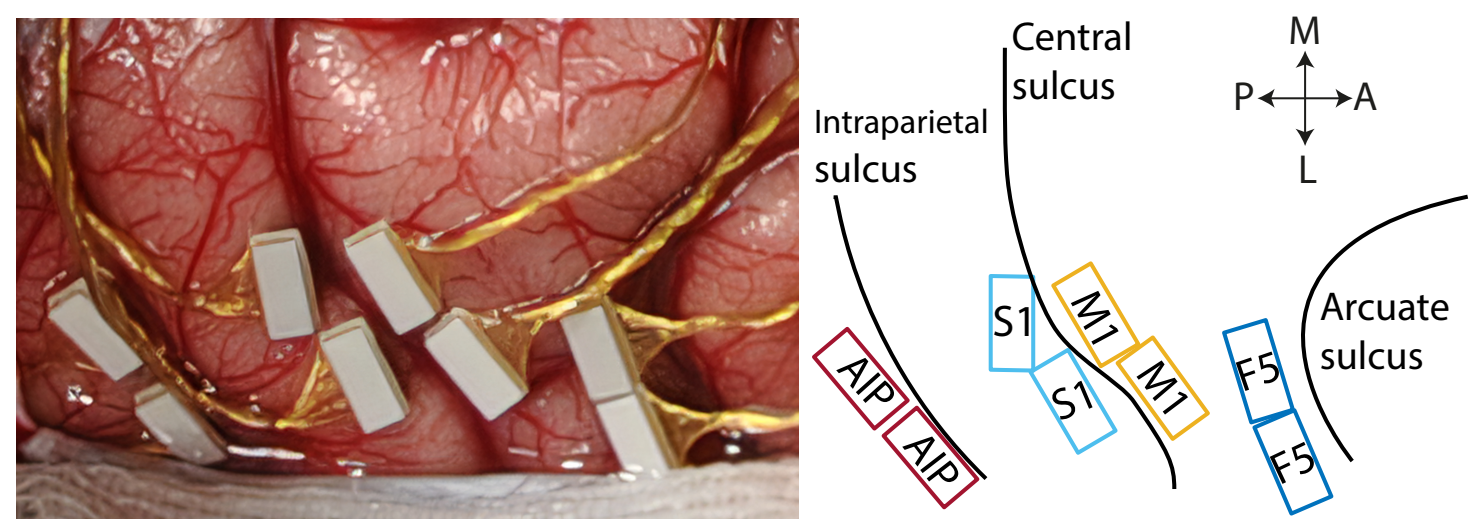

Figure 2.4: Position of all implanted arrays in monkey Ralph. Positions of all eight implanted arrays in monkey Ralph. Left: Intrasurgical picture, showing the white arrays on top of cortex, with gold cables leading away to connectors outside of the picture. Right: Schematic drawing of the implantation positions, highlighting landmark sulci and the area where each array is placed in.

Monkey Homer has been trained on the active grasping task since January 2016 but was not implanted at the time of finishing this thesis.

\subsection{Neural recordings}

In order to obtain data from the implanted areas (see 2.2), a neural signal processor was connected to the implants, while the monkey worked on his task in a room that was shielded from electric interferences from the outside. For this project I used two Neural Signal Processors (NSP) from Blackrock Microsystems Inc. (Salt Lake City, UT, USA), also known as Cerebus system. The first Cerebus system is an analogue system and is connected to an amplifier through a glass fibre cable. The system was placed outside of the setup, the amplifier inside and the connection cable was guided through a small hole in the setup to connect both. Both systems have a sampling rate of $30 \mathrm{kHz}$ at $16 \mathrm{bits}$. The amplifier featured four connector cables which can be connected to the implants through a female omnetics connector (Omnetics connector corp., Minneapolis, MN, USA) that fits the male connector at the end of each implant. The second Cerebus system is a digital system and is therefore connected to a digital hub inside the setup, again with a glass fibre cable. The digital hub also collects data from up to four 32 channel implants. At the 
end of each recording cable a small head stage is connected that ends in a female omnetics connector. The analogue amplifier and the digital head stage both preprocess the data from four 32 channel implants and send this information to the recording system. The recording system then saves the raw signal to a hard drive for later offline analysis.

During the recordings the ground of the digital hub and amplifier were connected to each other and the head post of the animal, to ground the hardware.

\subsection{Training}

B.F Skinner was one of the first scientists to propose that animals learn from the consequences of their behaviour in his theory of operant conditioning (Skinner 1990). If a behaviour results in a reward, such as access to food, it will be more likely that the animal will repeat this behaviour (positive reinforcement), while a negative consequence will make repeating this behaviour less appealing (negative punishment).

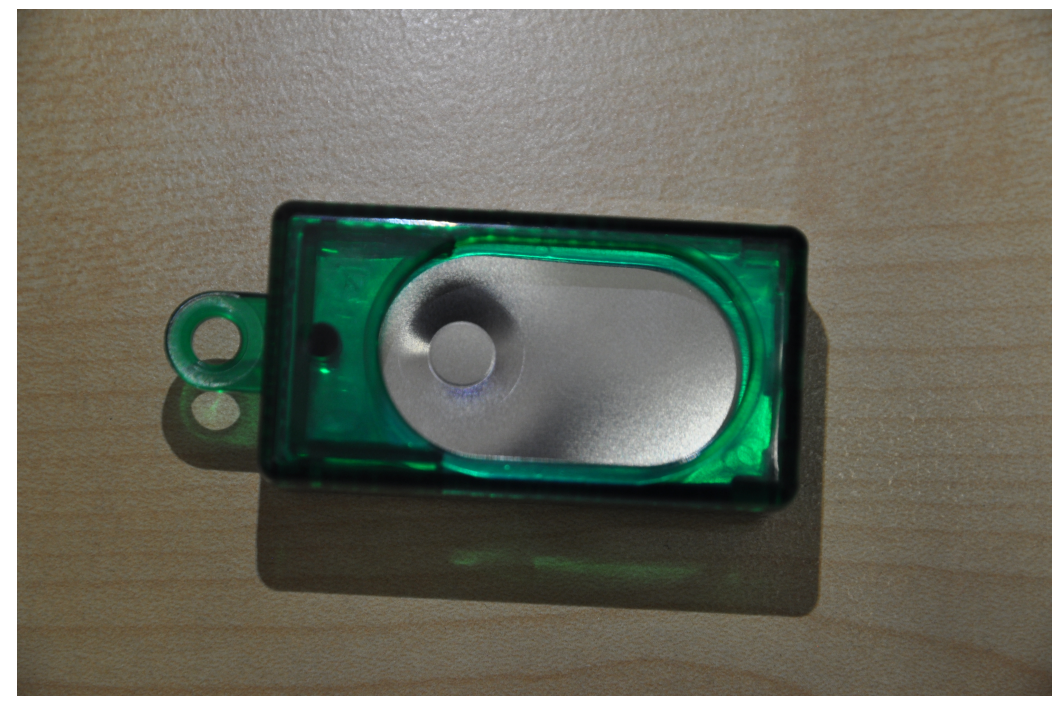

Figure 2.5: Picture of a manual clicker used for clicker training. A typical clicker used for clicker training, consisting of a small metal plate that will create a consistent clicking sound if pressed, inside a plastic case. Clicking indicates correct behaviour to a the animal.

Using this theory, positive reinforcement training (PRT) is nowadays an important tool to teach animals desired behaviours. When an animal is trained using PRT, correct behaviour is rewarded while wrong behaviour is neither rewarded nor punished but simply ignored. This way displaying the rewarded behaviour becomes more attractive to the animal, as it will expect another reward for repeating it. PRT has a wide range of possible applications in the laboratory setting ranging from encouraging movement to a different cage or simply as enrichment and management of undesirable behaviour (Baker et al. 
2009; Baker et al. 2010; Bloomsmith et al. 1998). For non-human primates, PRT is also a great tool to teach complex behaviours (Laule et al. 2003).

For this project, a variation of PRT was used, the so called clicker training (Feng et al. 2016). Here, correct behaviour is indicated by using the clicker before giving the reward. This is being referred to as a bridge (between behaviour and reward) or conditioned reinforcer (Gillis et al. 2012). A clicker is usually used for two main reasons: Firstly, it can create a very consistent sound with very small variation, therefore creating a very clear cue for the animal. Secondly, the clicker can be used very quickly, with just a small button press, therefore allowing for a very fast response to the behaviour, faster than grasping a reward and handing it over to the animal would ever be. This makes it very clear to the animal which behaviour was correct.

Both animals were only partially chair trained at the start of this project, meaning they were trained to enter a primate chair (custom build at the German Primate Center) on their own, but had no prior experiences with any kind of research task. Therefore it was necessary to teach all steps of the tasks to the animals, starting from general concepts like touching something in front of them to more complex parameters such as grasping twice when the light stays off, grasping only once when the light turns on. For some of these steps, smaller training equipment was build, such as a handheld object plate. This device was designed to look similar to the actual setup but was easily transportable and could therefore be taken into the animal facility and offered to the monkeys in their home cage. This way, they could familiarise themselves with the concept of the task without the constraints of a primate chair.

Both animals (section 2.1) were trained in a delayed-grasping task (section 2.5). Monkey R. was additionally trained in a passive finger stimulation task (section 2.6).

\subsection{Delayed-grasping or object recognition task}

To answer the question how sensory information can change the planning activity in the brain, both animals were trained in an active grasping task.

In this task, the animal was trained to grasp different objects that were perceived either visually or tactually beforehand. During the whole task, the animal sits in the dark and cannot see what is in front of him. A red LED light is used to cue the animal and instruct him to touch or lift the objects. The animal was trained to lift the object every time the cue light turned off. For the so called "tactile exploration phase" the monkey was given a few seconds to allow for exploration and recognition of the object and to find the best way to grasp it. The actual grasping phase was restricted to a few hundred milliseconds, 
to discourage further exploration and motivate the animal to plan his movement beforehand. Without planned movement, time would run out and the monkey would not be able to lift the object in time, since he would most likely bump into it at random locations.

\subsubsection{Experimental setup}

The setup for the active grasping task was build upon the turntable setup design used in Schaffelhofer et al. 2015. It features a round plate that can fit six different objects and a motor to rotate the objects to the front for presentation in (pseudo-)random order. Here, I modified the initial build with long front walls and small walls next to each object to restrict the view of the other five objects to ensure that only one object can be seen or touched at a time.

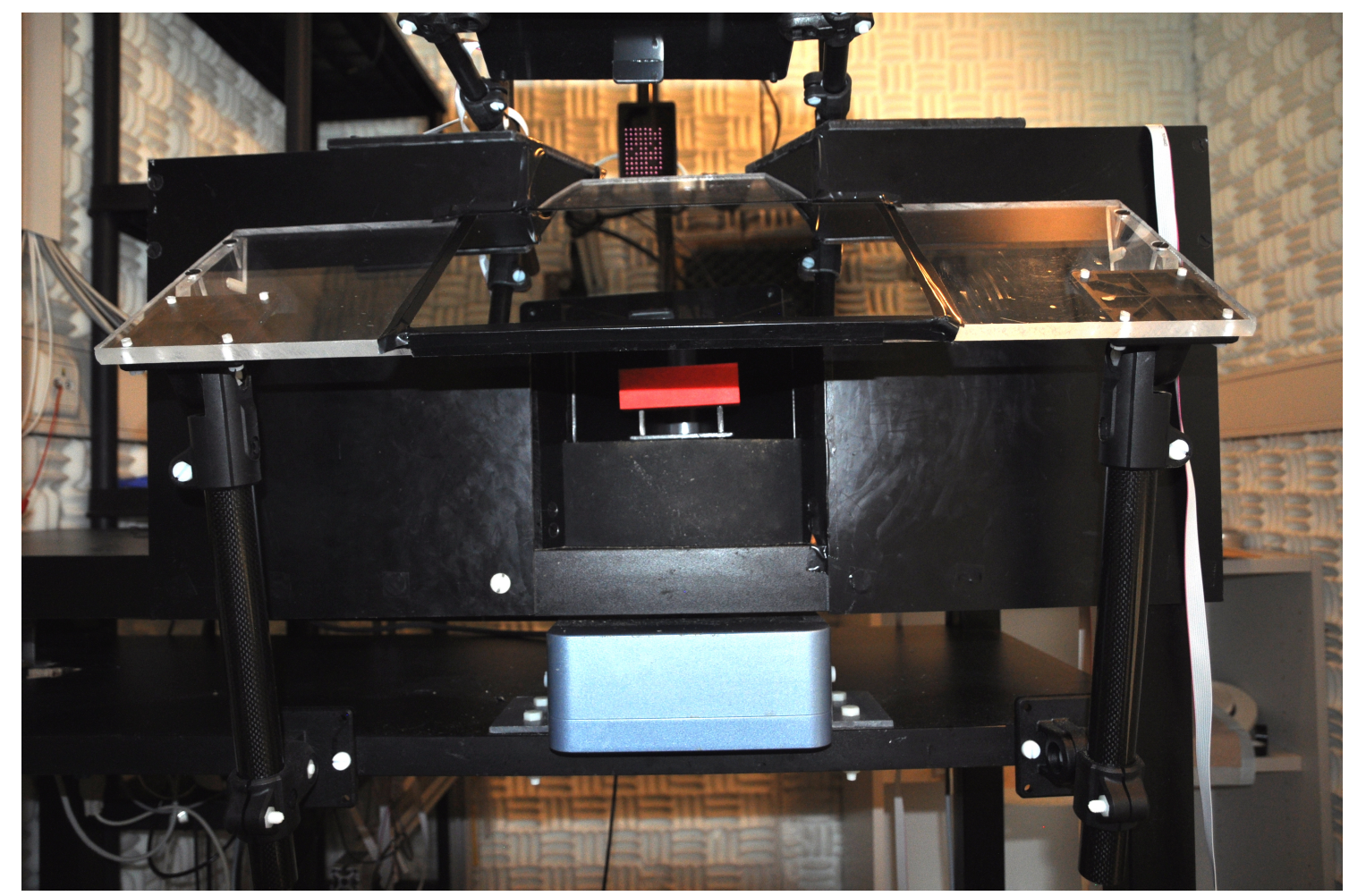

Figure 2.6: Front view of the turntable setup. Front view of the turntable setup used in this project. Visible are in red the current object (a cube) that has to be lifted, the halftransparent mirror on top of the object and the magnetic WAVE system in blue, placed under the setup.

For this experiment, the monkey was placed in the dark and could not see anything at the start of a trial. In tactile trials the monkey was instructed to touch the object and the light stayed off for the whole trial. In visual trials, a small illumination light for the object was turned on during the cue phase. A half transparent mirror was placed between the monkey and the object and the cue light was reflected onto the mirror. This way, the 
monkey could see the cue light clearly while looking directly at the object and did not have to split his attention. Below the setup, the WAVE system for the data glove was placed (see 2.5.3).

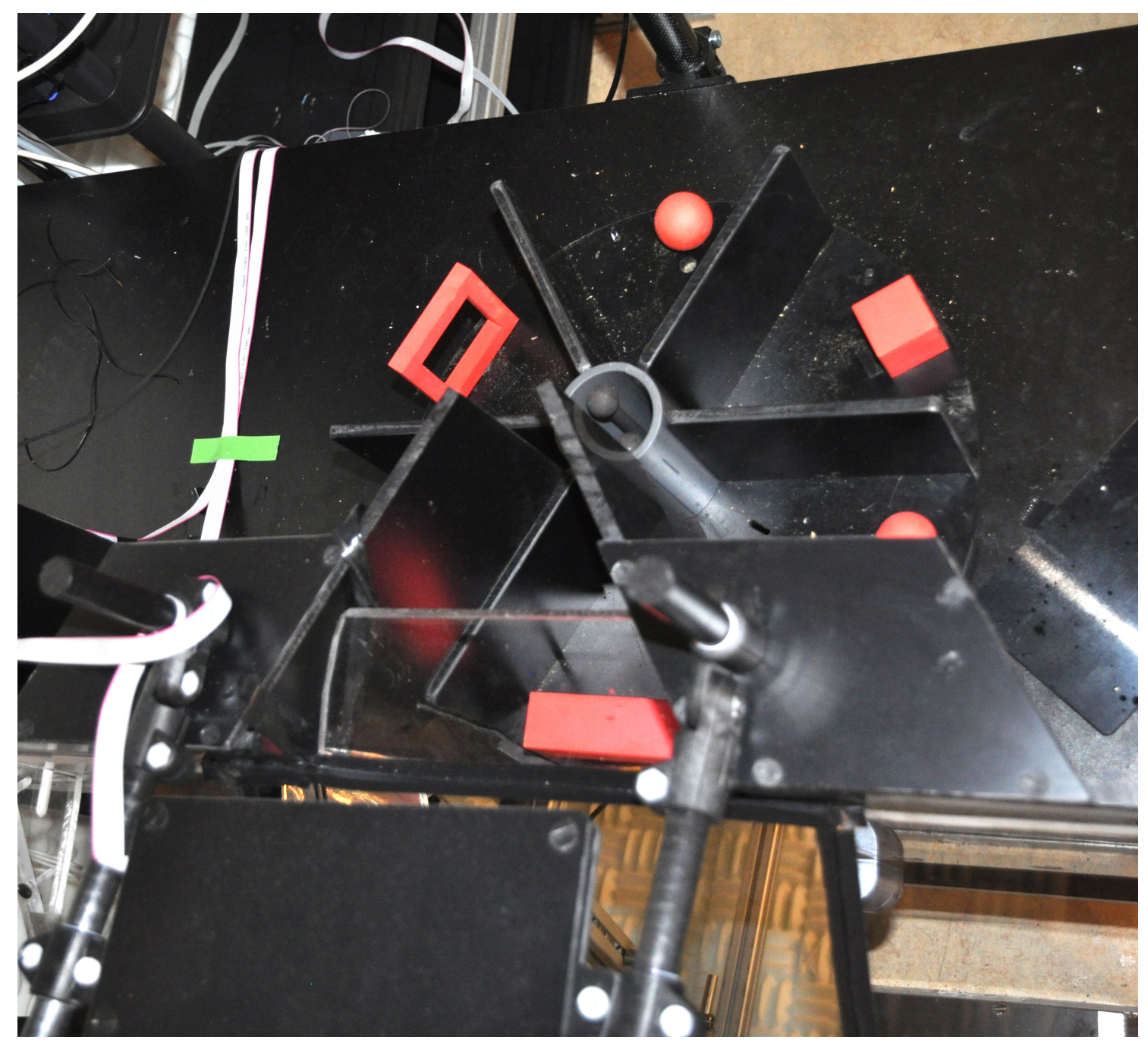

Figure 2.7: Top view of the turntable setup. View from the top of the turntable setup used for this project. Visible are five of the six objects (red) on the turntable plate that can be presented to the animal for grasping. The graspable object is in the lower end of the picture, below a half transparent mirror.

\subsubsection{Objects on turntable}

A crucial design question for a grasping task as the one described above is the design of the different objects to lift. All objects have a somewhat similar size and the same weight $(120 \mathrm{~g})$, to keep the effort to lift each object comparable. The weight is always a combination of the weight of the object and an additional block of plastic below the object that were connected with a carbon stick. Together these components weighted $120 \mathrm{~g}$. The object and the weights below the objects were designed in Autodesk Inventor 

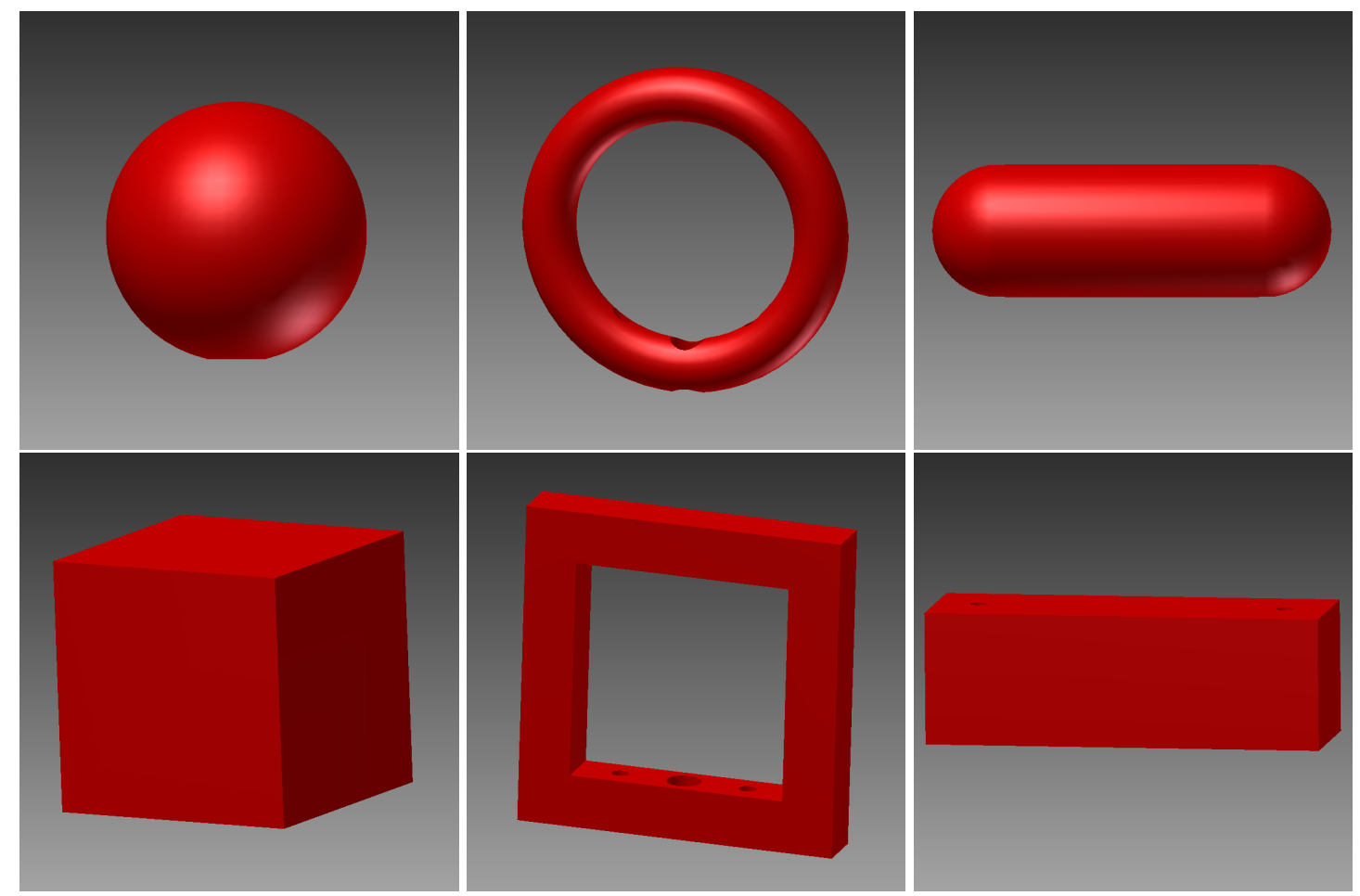

Figure 2.8: 3D renders of the six objects used in the delayed-grasping task. Top row: $A$ sphere, a ring, a rounded bar. Lower row: A cube, an edged version of the ring and a box. Objects in the same column require roughly the same hand shape to be grasped.

(Autodesk, Inc.) and 3D printed out of plastic (PA 2200 by Electro Optical Systems GmbH, Munich, Germany) by Shapeways (Shapeways Inc., New York, United States). After arrival, they were mounted onto the plate and the different parts were merged together with super glue (Loctide, Henkel AG \& Co. KGaA, Düsseldorf, Germany). The length of the carbon stick was adjusted so that each object had to be lifted by $1 \mathrm{~cm}$.

Figure 2.8 shows the object shapes that were designed to encourage tactile exploration and punish quick grasps without former knowledge by requiring a variety of different hand shapes. Objects were designed in a way that covered a variety of different grasps and different forms.

\subsubsection{Recording of hand kinematics}

An important step in correlating hand movements with neural activity is accurate measurement of hand position during the task. Here, I was especially interested in quantifying whether visually and tactually guided grasps are the same or if the monkey would come up with different strategies or forms the hand differently, to successfully grasp the same objects.

Due to constrains of the setup design, such as separators between objects to avoid that 
the animal sees multiple objects at a time, tracking systems that require a line of sight could not be used. Instead I used a kinematic data glove for small primates that had been previously developed in our lab (Schaffelhofer and Scherberger 2012, see figure 2.9).

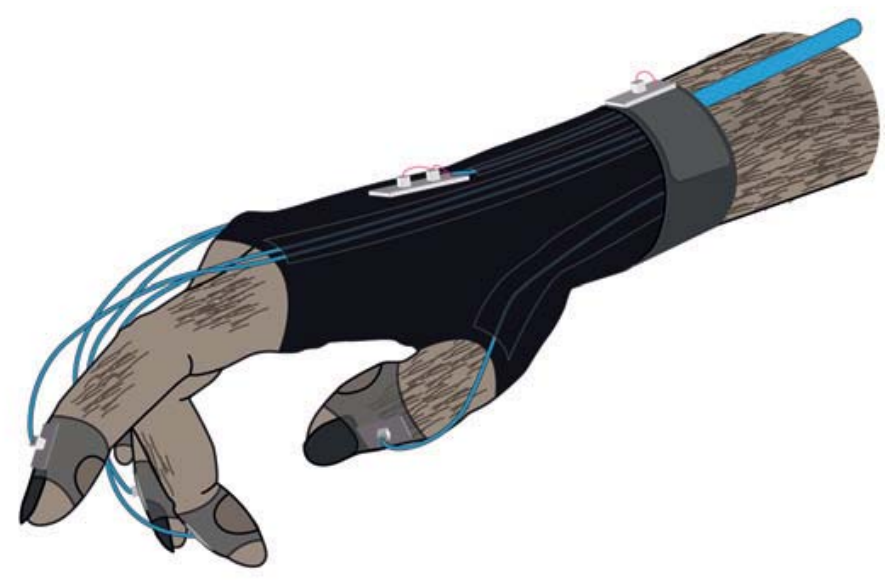

Figure 2.9: Illustration of the kinematic data glove. Schematic drawing of the glove, showing seven sensors at the finger tips, hand dorsum and wrist and the cables leading signals away from the sensors. Illustration first appeared in Schaffelhofer and Scherberger 2012)

This data glove utilises a magnetic field in order to track the position and orientation of attachable markers with a sampling rate of $100 \mathrm{~Hz}$, allowing it to be used even in heavily occluded setups. The magnetic tracking system (Wave, Northern Digital Inc. Waterloo, Canada) was placed below the setup, in a position that allowed to track the hand movements during grasp and in hand rest position to allow observation of the whole hand trajectories. Seven sensors were used to track 32 degrees of freedom of the hand and arm movements, one was attached on top of each finger nail, one on the back of the hand and one on the wrist.

In order to use this glove, the animal needed to be trained to wear a customfitted data glove made out of fabric which holds part of the sensors in place (hand dorsum and wrist) and serves as a place to secure the cables coming from the sensors on the finger tips. The glove spans the hand from the end of the fingers to the start of the wrist and is secured on the wrist using velcro (see figure 2.10, left). The sensors on top of the finger nails are secured using super glue (UHU GmbH \& Co. KG, Bühl, Germany) between finger nail and sensor and Leucoplast (BSN Medical, Hamburg, Germany) on top of the sensors (see figure $2.10 \mathrm{~B}$ ). The Leucoplast serves as a fail-save mechanism in cases when the super glue would not bind correctly. The Leucoplast did reach from one side of the finger tip to the other, above the sensor, so that the finger tips itself were free and the sense of touch was not blocked. 

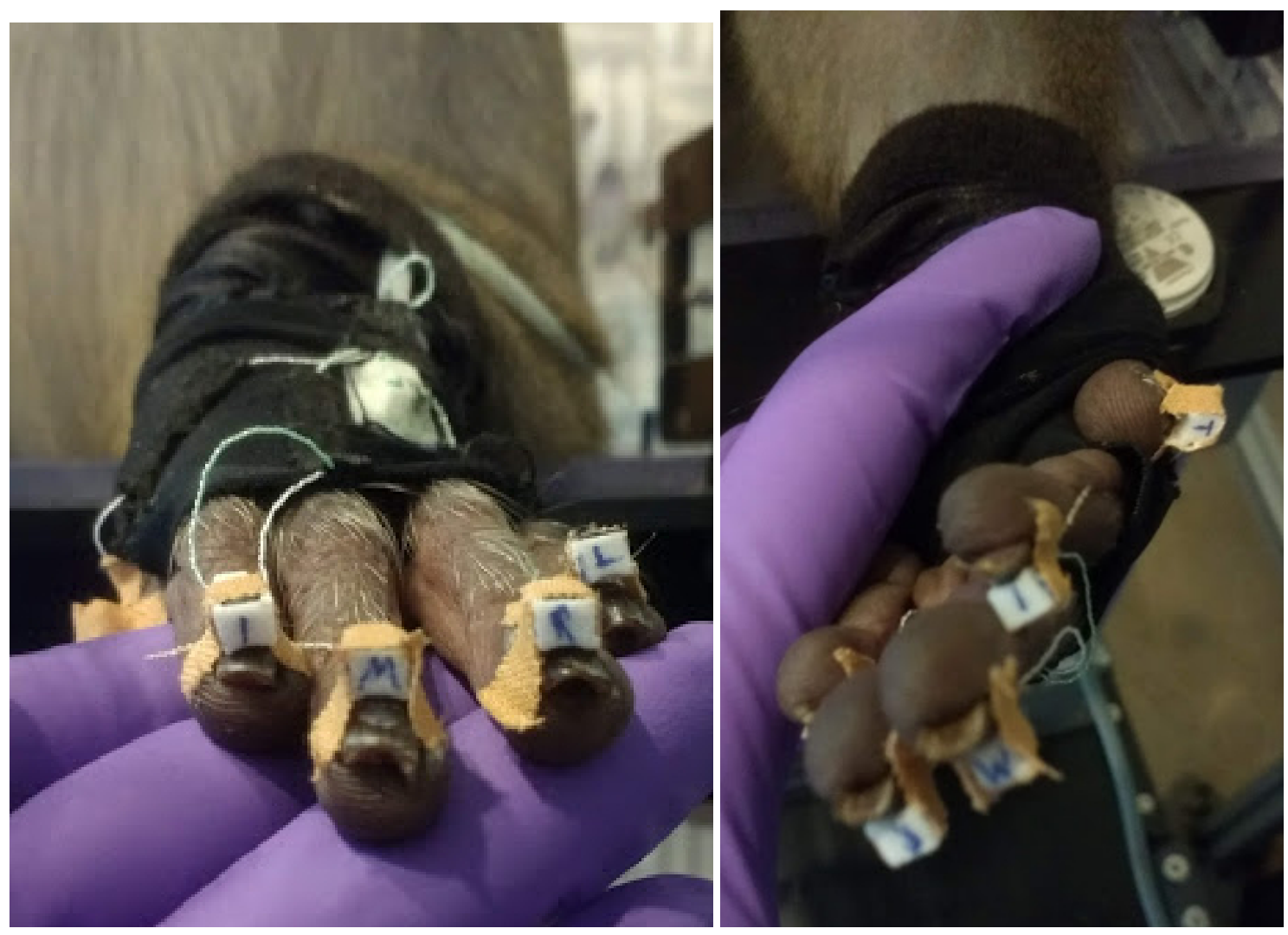

Figure 2.10: Photos of the kinematic data glove, worn by monkey Ralph. Kinematic data glove (Schaffelhofer and Scherberger 2012). Pictures of monkey Ralph wearing the data glove, while the sensors (white and marked with letters) are glued to his fingertips using Leucotape. The Leucotape secures the white sensors from the top but leaves the finger tips free. Purple: The experimenters hand.

The resulting data was saved to a hard drive for later offline analysis. A synchronisation signal was sent to the NSP to allow correlation of movements with neuronal activity. To get a first impression of the movements a visual analysis of the movement data between trials was conducted, comparing possible difference between grasping of the same object for the same and between both sensory conditions. 


\subsubsection{Behavioural paradigm}

As described in section 2.5.1, six different objects are presented on a rotatable turntable. During the whole experiment, the animal sits in the dark and initiates a trial by placing its hand on a hand rest button. See figure 2.11 for a schematic drawing of the task paradigm, indicating the different epochs of this experiment as well as the length of all epochs.

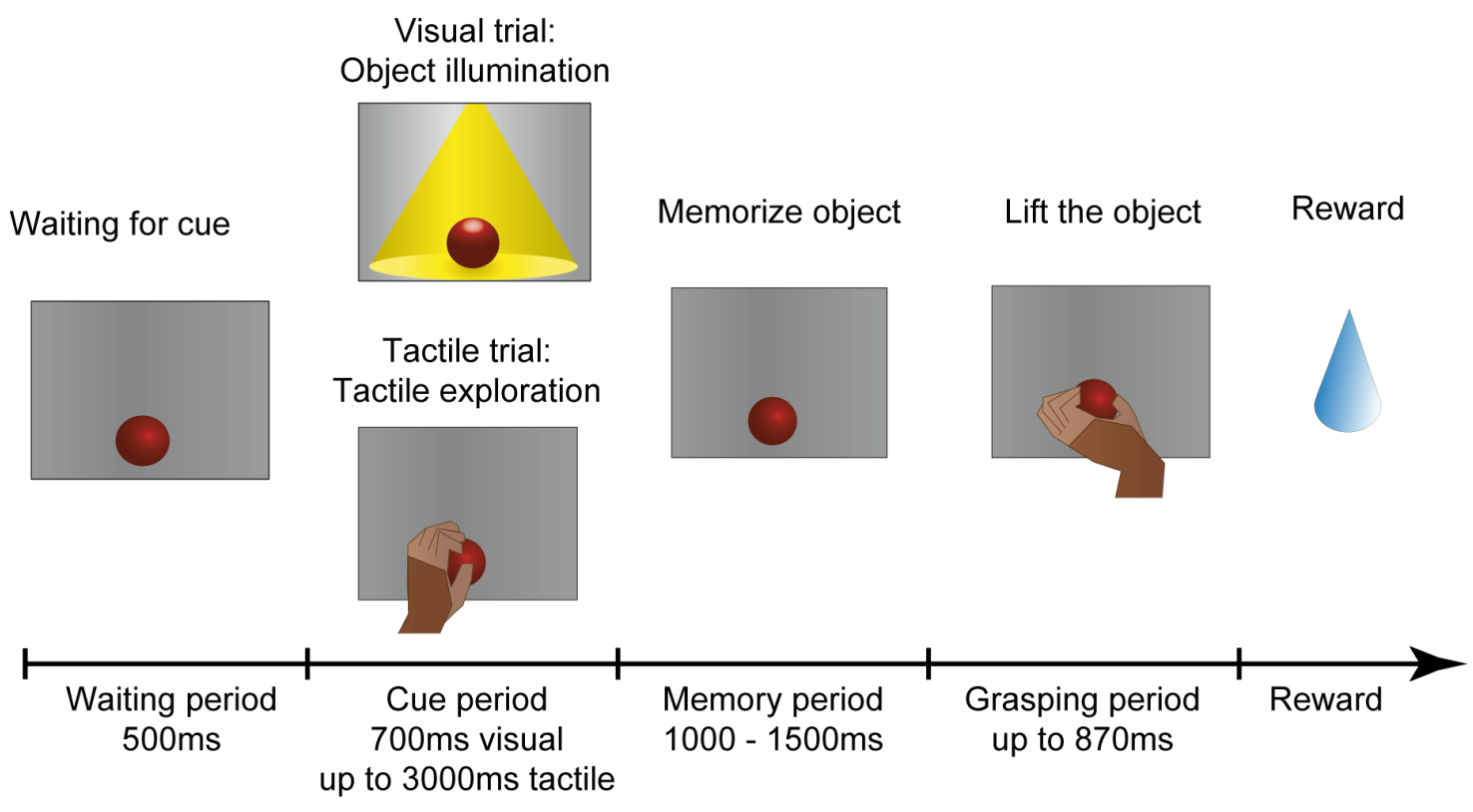

Figure 2.11: Illustration of the task paradigm. An overview over the task paradigm. First, the animal has to wait for $500 \mathrm{~ms}$ for the cue. The object is either illuminated for $700 \mathrm{~ms}$ (visual trial) or the animal is instructed to tactually explore the object (up to $300 \mathrm{~ms}$ before a trial is aborted). The memory period that follows has a randomly chosen length between 1000 and $1500 \mathrm{~ms}$, before the animal is instructed to lift the object. Grasps lasting longer than $870 \mathrm{~ms}$ are again marked as error and failure to lift in time.

The handrest button encourages the monkey to keep a similar starting position for each trial, with his full hand placed on the button and also ensures that the animal does not reach into the moving turntable, as the motor will stop as soon as the button is released. At the start of each trial, the turntable will be turned to one of the objects, which are presented in pseudorandom order, to ensure an equal number of grasps per object. After one of the objects arrives in front of the monkey, a red LED is switched on. The monkey has learned to observe this LED and grasp the object in front of him, whenever it turns off. Afterwards, either a visual or a tactile trial will start. In the next period, he either has to reach out to touch it and confirm the touch with a short lift or he has to sit still and the object is illuminated for $700 \mathrm{~ms}$ instead. For the touch and lift, the animal has $3000 \mathrm{~ms}$ to successfully lift the object, before the trial is aborted. After either seeing or touching the object, a variable memory period starts lasting between 1000 and $1500 \mathrm{~ms}$, to avoid that the animal will guess the appearance of the Go cue and instead has to observe the 
LED. After the memory period ends, the red LED will turn off and the animal has $870 \mathrm{~ms}$ to grasp and lift the object. The first 70ms stem from a reaction time period, during which the animal can not lift the object without having guessed, calculated from the average reaction times during early training. Trials in which the object is lifted inside those $70 \mathrm{~ms}$ were marked as false. This never happened during actual recording, showing that the animal actually waited until the LED turned off and did no longer try to guess the Go cue. After successfully grasping the object, the monkey had to return to the hand rest position to receive a fluid reward.

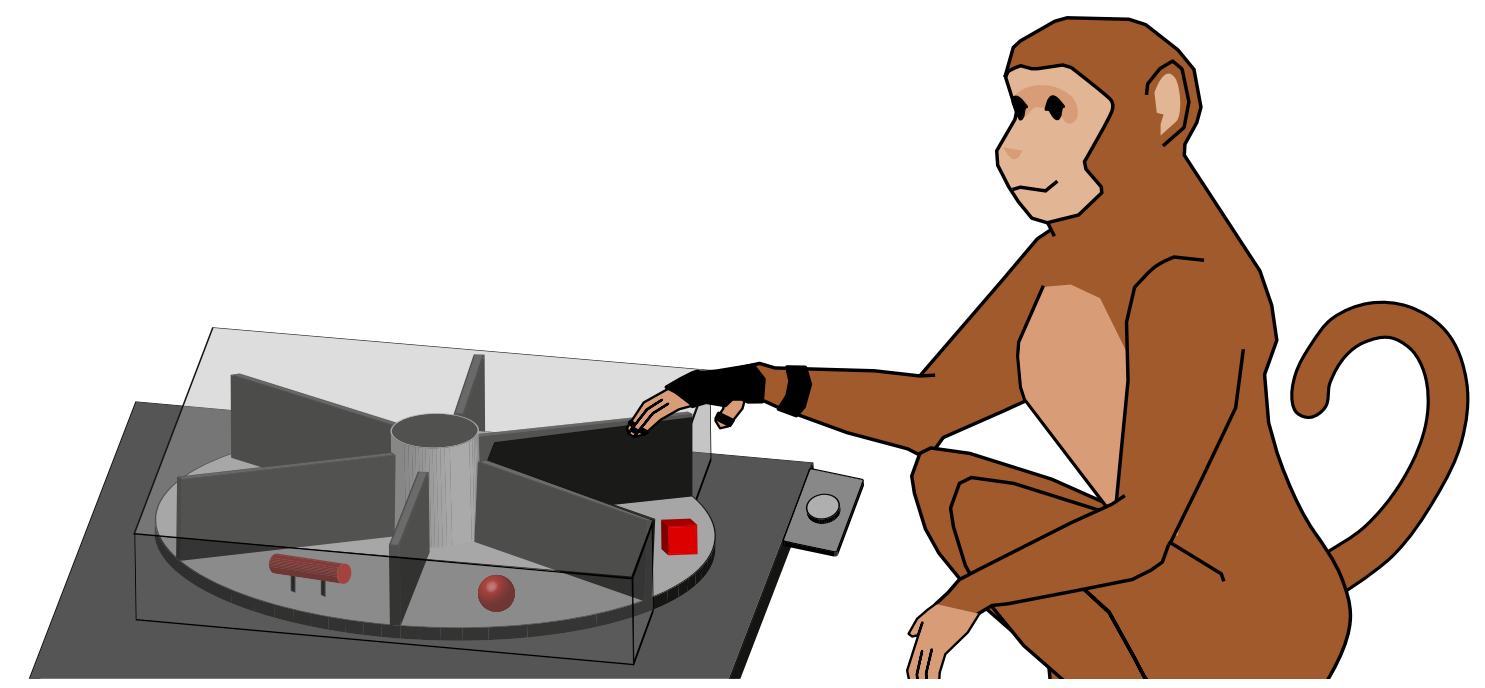

Figure 2.12: Illustration of an monkey working on the turntable. The monkey working on the turntable (simplified). The turntable consists of a plate (light gray) that features six different objects (red). The object in front of the animal has to be lifted, while the other ones are out of view (illustrated by a cover). The animal wears a black data glove. Between animal and turntable, a light grey handrest button is visible.

\subsection{Passive stimulation experiment}

With the passive stimulation experiment I aimed to investigate how different structures are represented in the four brain areas (see section 2.2), when they are passively presented to one finger.

\subsubsection{Experimental setup and behavioural paradigm}

For this task, the animal has to extend one finger and hold still, while a mechanical platform presents different stimuli from left to right or right to left to the finger. Between stimulation trials, the animal is rewarded with a liquid reward, encouraging it to sit still during the trials. 
The setup consists of a mechanical platform (for a description see Oddo et al. 2011) that can move stimuli that are mounted on small plates up and down with a preset consistent force and left and right with a preset velocity. For this experiment, a force of $600 \mathrm{mN}$ and $25 \mathrm{~mm} / \mathrm{s}$ were chosen. These values kept stimulation pleasent for the animal while minimizing trial time to avoid that the monkey gets impatient. See figure 2.13 for a photography of the platform.

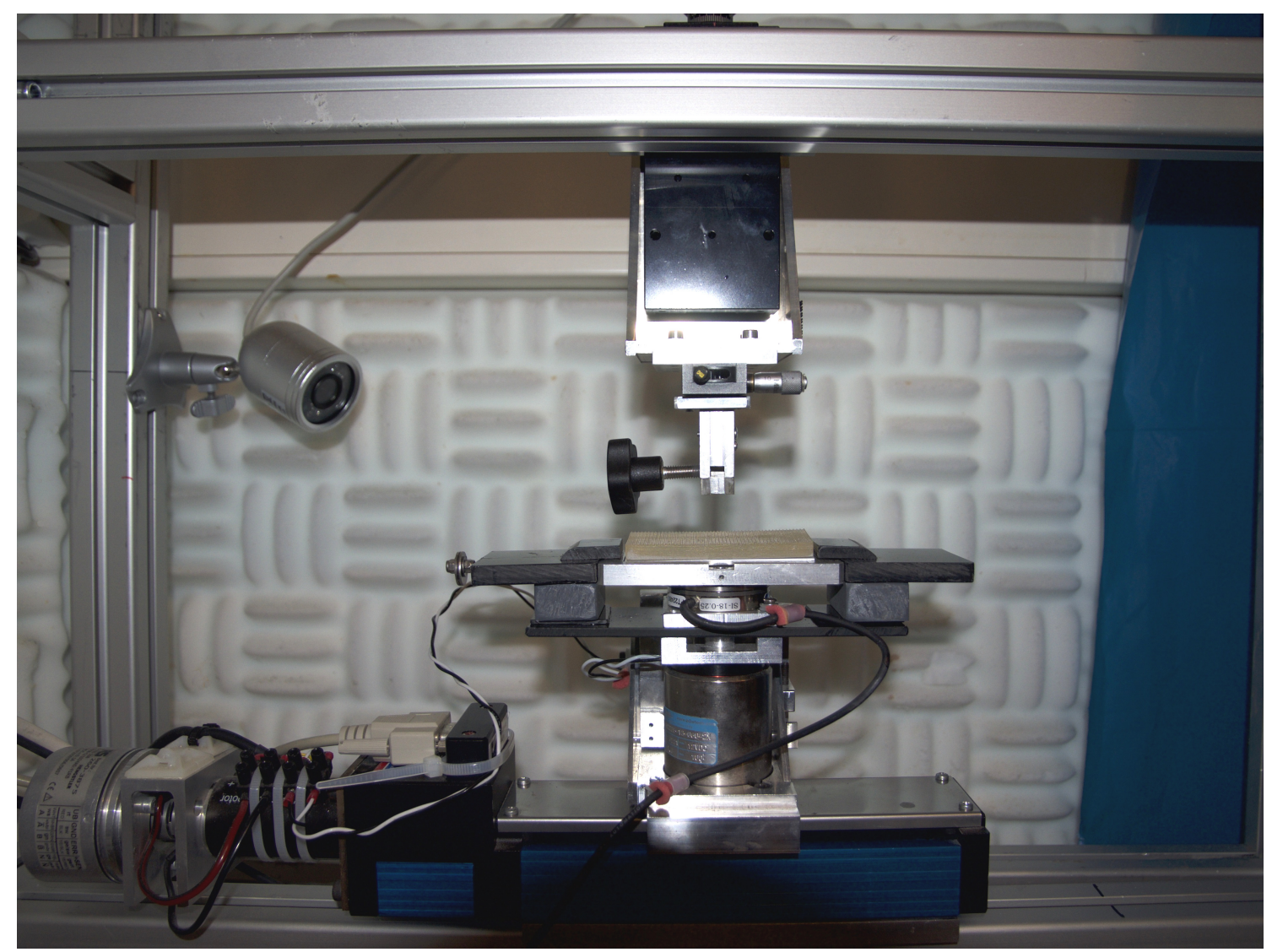

Figure 2.13: Image of the moveable platform used in the passive stimulation task. Photo of the platform that is used in the passive stimulation task. The stimulus (beige) is placed on the platform. A plastic finger holder will be placed into the silver slot above the stimulus, where the finger can be fixated. A motor (left) moves the platform from left to right, while a voice-coil actuator moves the platform up and down. A force sensor below the stimulus measures the executed force to adapt it should disturbances occur, to ensure a consistent force. 

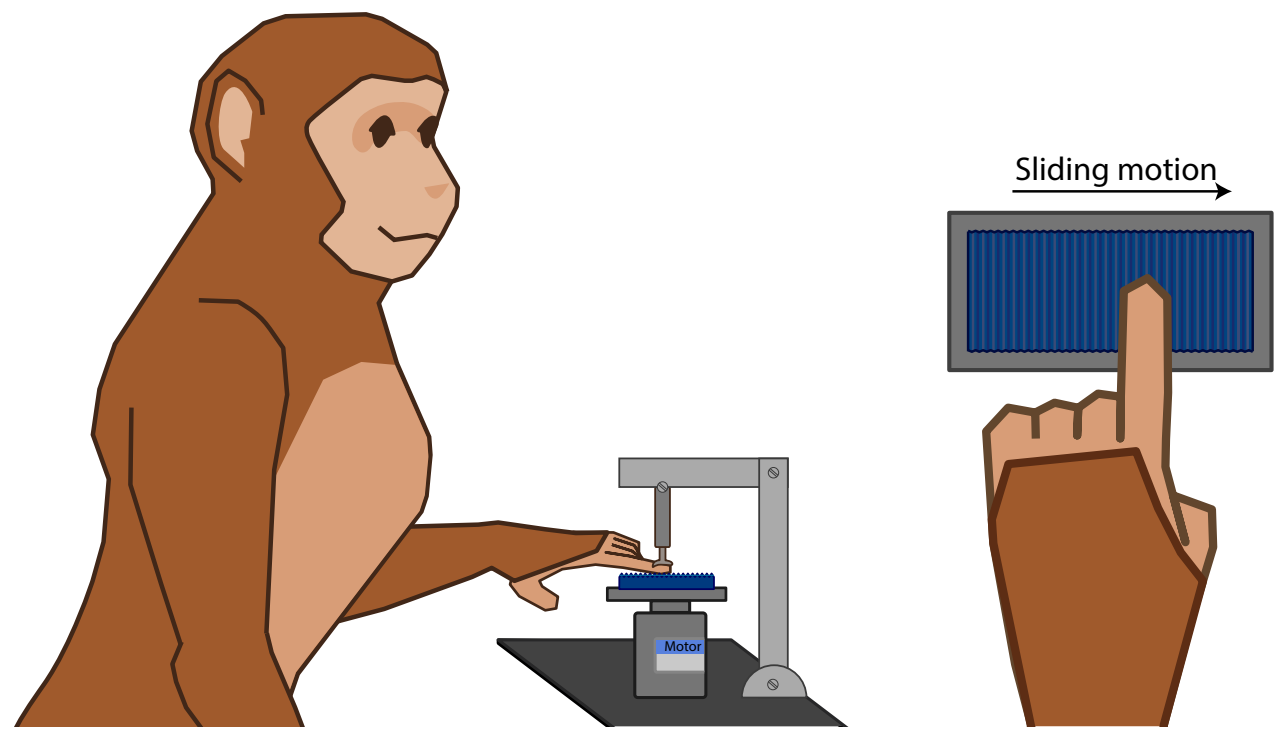

Figure 2.14: Illustration of the passive stimulation task. An illustration of an animal during the passive finger stimulation task. Left: The monkey sits in front of the platform (gray) used in the passive stimulation task (simplified). He extends his fingers, which is fixed to a finger holder, holding it in place. The platform moves a structured plate (right image) up to the finger from below, then either in a rightward or leftwards movement direction below the finger of the animal and then is lowered again.

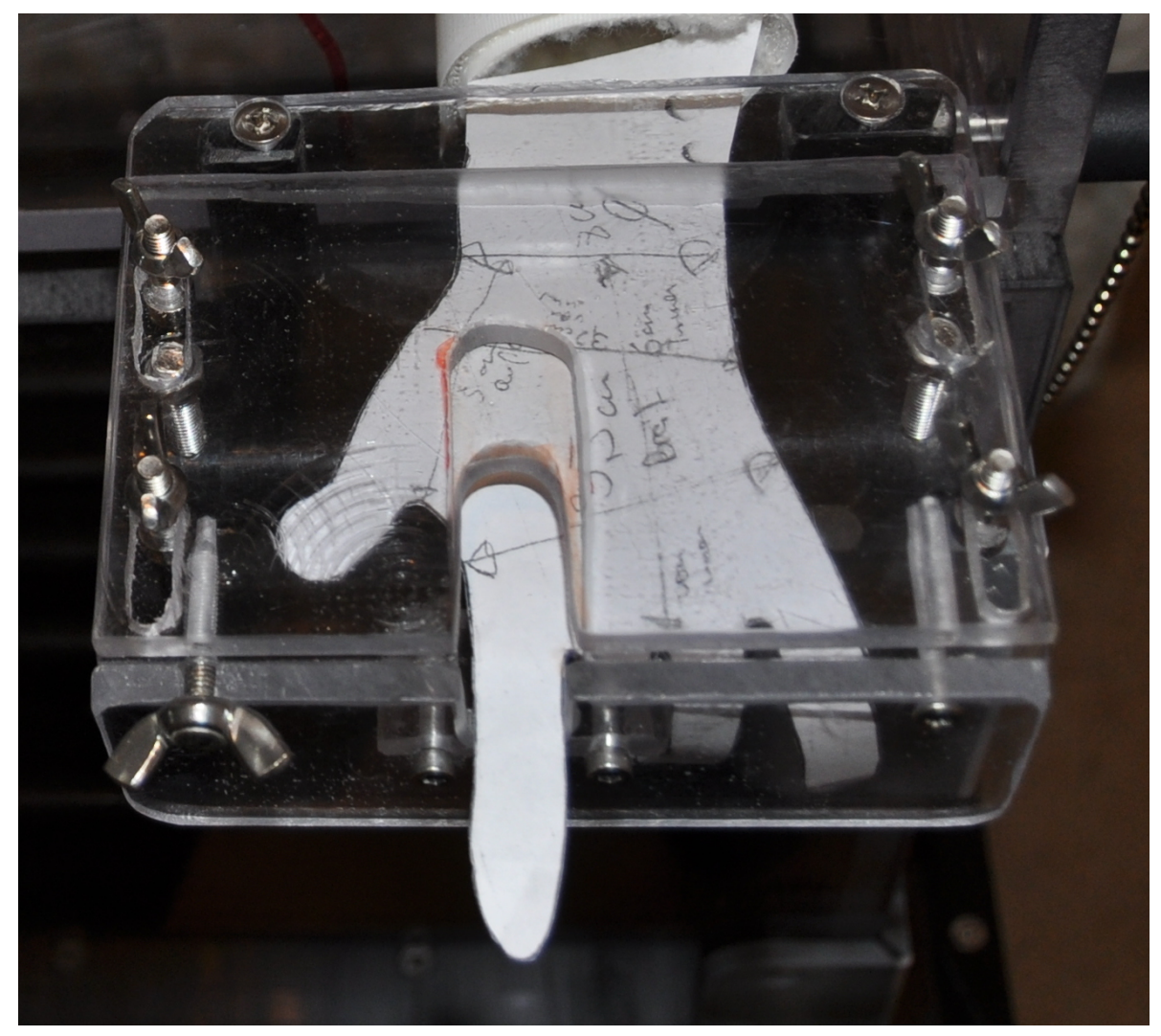

Figure 2.15: Photo of the hand holder. A photography of the hand holder. The animal places his hand around a bar (below the paper hand) and has to extend one finger through the hole at the front of the hand holder. Plates around the hand restrict movements to avoid that the animal accidentally comes into contact with moving platform parts. 
The animal is placed in front of this platform and has to extend his finger, as illustrated in figure 2.14. Since the platform was originally designed for use on human patients, I chose the longest finger, which was the middle finger. Further considerations went into which fingers might be most sensible, to achieve a good amount of stimulation. The hand was placed in a custom build hand holder which consists of a bar to grasp around with the remaining fingers for comfortable positioning and additional plates that restrict movement to avoid that the monkey grasps into the mechanics. A finger sized hole in the front of the hand holder allowed the monkey to peek his finger through the holder. The finger was then fixed with a drop of super glue (Loctide, Henkel AG \& Co. KGaA, Düsseldorf, Germany) on the finger nail to the top of a finger holder, to further restrict movement and to hold the finger in a consistent position.

A force sensor measured the current force that was applied on the finger from pushing the stimulus onto the finger pad and adjusted it in case of movement (such as the monkey pushing downwards) to keep the overall force at the desired level. A structured plate was pushed with a preset force against the finger, moved either from right to left or left to right (pseudorandom). The plates were presented in block design, meaning they were only changed after 20 trials of each movement direction were completed, since changing the plates required entering the setup and manually switching it out.

\subsubsection{Stimuli}

To cover a variation of different structures, five different stimuli were used, featuring a range of different gratings.

The set consisted of a small bar with a ridge width of 250 micrometers placed at different distances, ranging from 500 to 3000 micrometers, with different distances left and right on most plates (see figure 2.17).

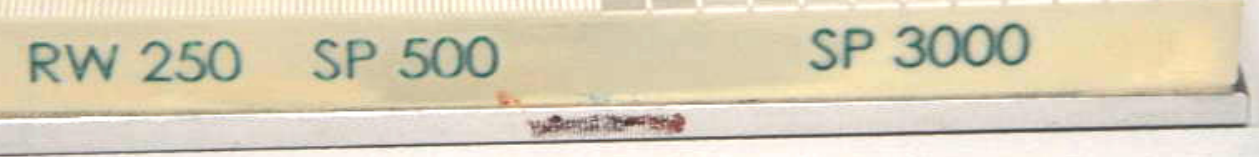

Figure 2.16: Close-up of one stimulation plate used for the passive stimulation task. The different grated stimuli used in the passive stimulation task. Ridge width for all stimuli was $250 \mu \mathrm{m}$. Each plate had a different spacial pacing on the two sides. Spacial pacing of the shown stimulus was $500-3000 \mu \mathrm{m}$ 


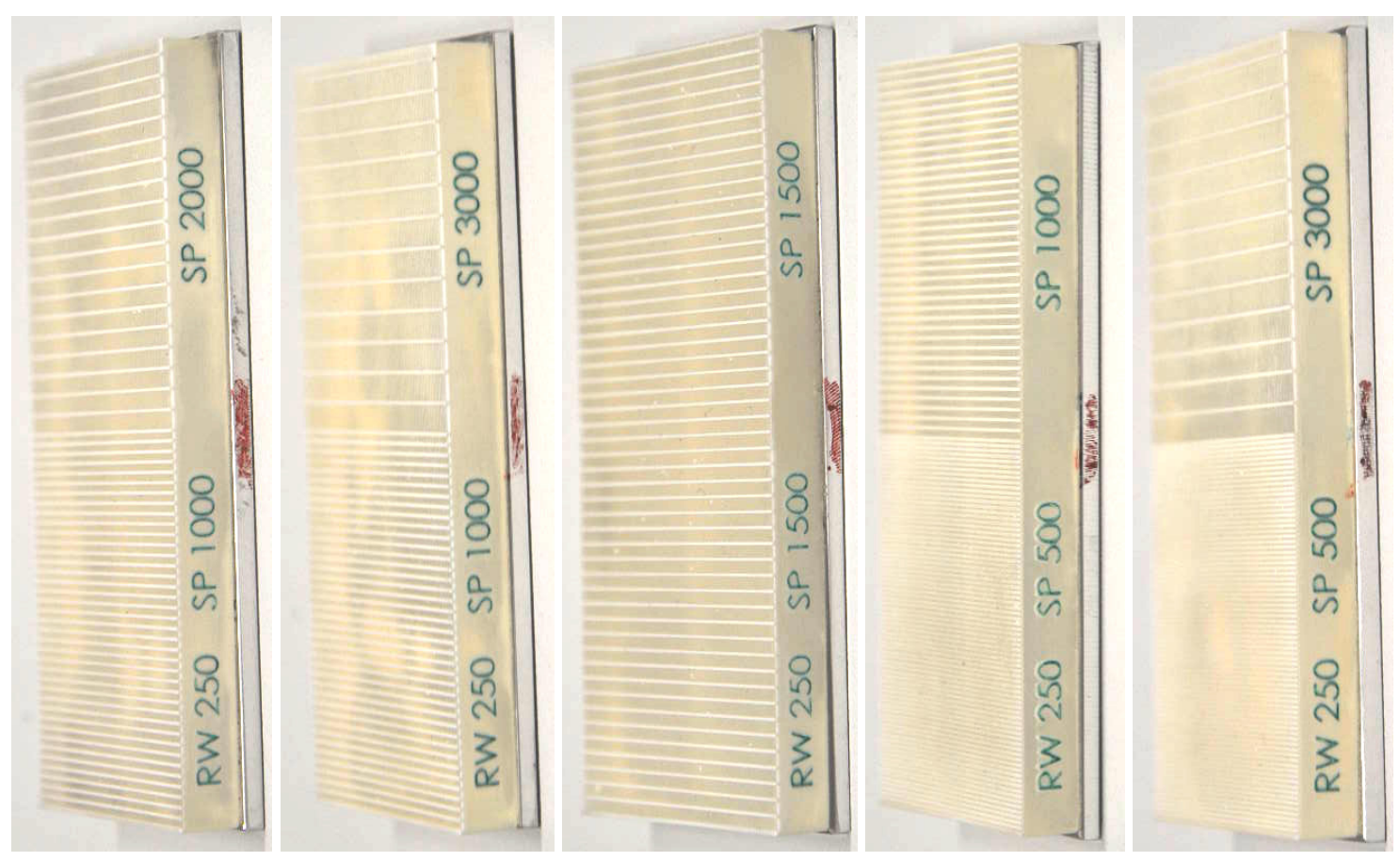

Figure 2.17: Photos of the stimulation plates used for the passive stimulation task. The different grated stimuli used in the passive stimulation task. Ridge width for all stimuli was $250 \mu \mathrm{m}$. Each plate had a different spacial pacing on the two sides. Spacial pacing (left-right) for the five stimuli was $1000-2000 \mu \mathrm{m}, 1000-3000 \mu \mathrm{m}, 1500-1500 \mu \mathrm{m}, 500-$ $1000 \mu \mathrm{m}$ and $500-3000 \mu \mathrm{m}$
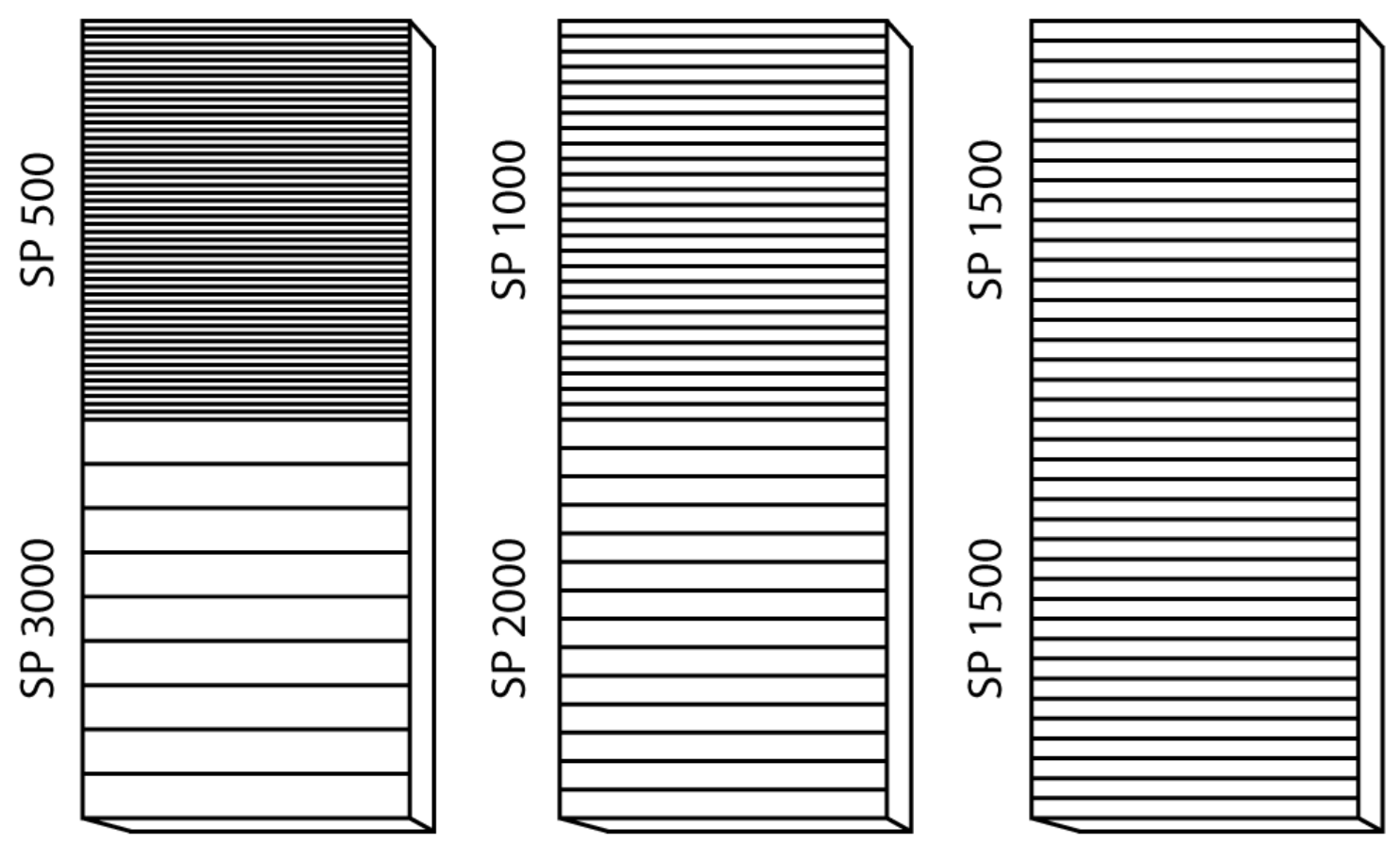

Figure 2.18: Example illustration of three stimulus plates. An example illustration of three stimulus plates to better illustrate the different gratings used. Spacial pacing (leftright) for these three stimuli was $3000-500 \mu \mathrm{m}, 2000-1000 \mu \mathrm{m}$ and $1500-1500 \mu \mathrm{m}$ 


\subsection{Data analysis}

All data analysis for this project was conducted in custom made scripts written for Matlab (The MathWorks, Inc., Natick, MA, USA), unless specified differently.

\subsubsection{Spike sorting}

After raw data acquisition (see section 2.3), data was prepared for detection of spikes. In a first step, data was filtered using a median filter with a window length of $3.33 \mathrm{~ms}$. The result of this filtering was then subtracted from the raw signal. Afterwards, a 4th order non-causal Butterworth filter $(5000 \mathrm{~Hz})$ was applied to low-pass filter the data (Butterworth et al. 1930). Channels that displayed obvious noise during recording were usually noted down during recording and taken out of the analysis and not processed further. Less than seven channels per day were considered unusable due to noise.

Certain noise, such as noise due to movements of the animal will be common on all channels on each array. In order to eliminate these common sources of noise, common components were identified. Afterwards, principal component analysis (PCA) artefact cancellation was applied to each channel, as described in Musial et al. 2002. Any PCA dimension that had a coefficient greater than 0.36 (with respect to normalized data) was kept, to avoid removing individual channels.

For the passive finger stimulation task, spike waveforms were extracted using a modified version of the offline spike sorter Wave_clus (Chaure et al. 2018; Kraskov et al. 2004). The extracted spike waveforms were then used for further analysis and will be referred to as multiunits. No further spike sorting was prepared for this project.

For the active grasping task, six different recording sessions were used for the analysis and further spike sorted. After preparation of the data as described above, Wave_clus (modified as mentioned above) was used to extract the spike waveforms and sort them.

\subsubsection{Movement time analysis}

To assess whether or not the animal is using the cues presented to him before grasping the object, an evaluation of the time he needs to react to the go cue and complete the movement was done. Since the objects were designed in a way to encourage very different grasp types (see section 2.5.2), grasping an unknown object should take longer than grasping a known object. Chance level of guessing the correct grasp type without using the object information would be $33,3 \%$ since three different grasping types were present on the turntable (six objects, from which two encouraged the same grasping type). This 
means that the animal would only be able to guess the grasp type correctly in one third of the trials and would have to try a different strategy in two thirds, which increases movement time. Reaction times were measured from the appearance of the Go cue, till the monkey leaves the handrest button. Movement times were measured from appearance of either Touch or Go cue (depending on the task) to the moment the object is fully lifted and compared between visual and tactile trials.
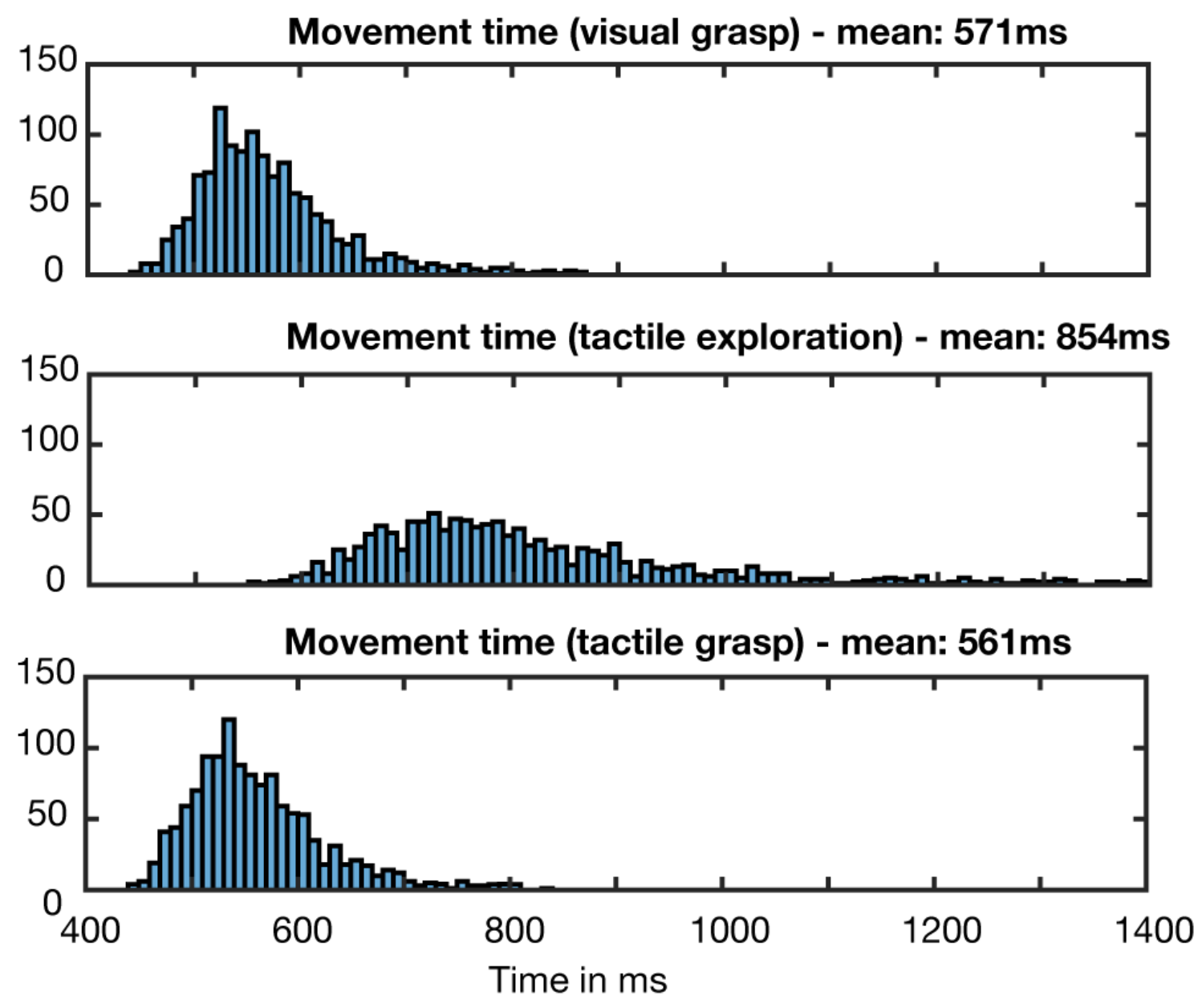

Figure 2.19: Example comparison of movement times. Example comparison of different movement times. Plotted is the number of occurrences of movement times (bin size: $10 \mathrm{~ms}$ ) as a histogram for visual trials during grasp and for tactile trials during tactile exploration and grasp. Movement times were measured from appearance of touch (for tactile exploration) or go cue until the object was successfully lifted.

The reaction and movement times are then plotted as a histogram, where the number of occurrences of a certain movement time is plotted during grasp and tactile exploration. For movement times, a bin of $10 \mathrm{~ms}$ was chosen, for reaction times a bin of $5 \mathrm{~ms}$, due to the smaller range of values. The first panel always shows the corresponding times for visual trials, during the grasp period. The second panel covers the reaction or movement times during tactile exploration of an object, for tactile trials. The third panel shows the times for the grasp epoch of tactile trials. 
Trial numbers were pooled over all recording days to get a better representation of the animals behaviour over time.

\subsubsection{Peristimulus time histogram}

A peristimulus time histogram (PSTH) was used as a way of plotting the average fire rate of neurons over time. After extracting and sorting spike waveforms (see 2.7.1), the spike trains were stored at a resolution of $1 \mathrm{~ms}$. Afterwards, average activity was calculated and a Gaussian filter was used to smooth them.

Due to the different nature of the two tasks (Active grasping task and passive stimulation task), different ways to plot the data were chosen.

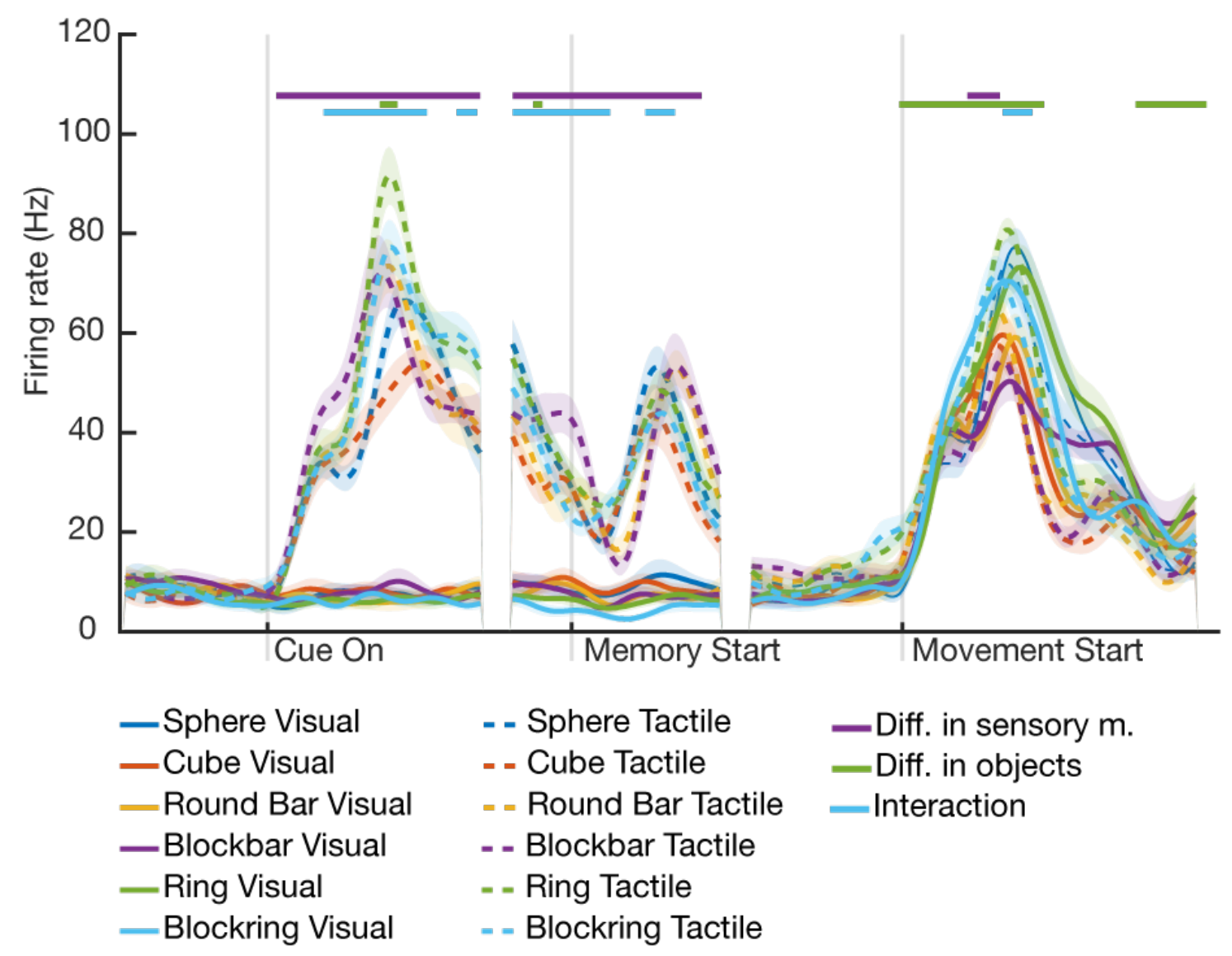

Figure 2.20: Example PSTH of an unit in primary motor cortex during active grasping task. PSTH of an unit in primary motor cortex during active grasping task. Plotted is the firing rate in $\mathrm{Hz}$ over time. Each colour corresponds to one object, solid and dashed lines mark visual and tactile trials, respectively.

Since the task design of the active grasping task (section 2.5 ) required some variable time ranges (such as memory period lasting between $1000 \mathrm{~ms}$ and $1500 \mathrm{~ms}$ to avoid that the monkey estimates the appearance of the Go cue) the spike trains were aligned at three 
different time periods: Start of Cue period, start of memory period and movement start. Figure 2.20 shows an example PSTHs for a unit during the active grasping task.

For visual trials, start of cue period was defined as the moment the object was illuminated and visible to the monkey. For tactile trials, Cue onset was instead defined as the onset of the Touch cue, when the red LED turned off and the monkey was instructed to now touch, explore and lift the object. 500ms of signal were plotted before start of Cue, as this would refer to a baseline activity, where the animal is not yet doing anything, is not moving (his hand rested on the hand rest button) and cannot see the object, yet. After start of cue, $1000 \mathrm{~ms}$ were plotted to observe how the input of visual or tactile information changed the firing rate.

The second alignment point, start of memory period, was defined as the end of the cue period, where the cue completely disappeared. For visual trials, the start of the memory period was defined as the moment the light turned off and the monkey could no longer see the object. For tactile trials it was when the animal returned to the hand rest button and stopped to move completely. While the actual tactile cue did stop earlier (when the monkey released the object and started moving his arm back to the hand rest button) the hand rest button provided a reliable signal that ensured that all tactile stimulation from the object had ceased. Here, $100 \mathrm{~ms}$ before memory period starts were plotted and $500 \mathrm{~ms}$ after. The $100 \mathrm{~ms}$ were to observe the direct change in activity that happens when the light turns off or the movement stops.

The last alignment point was the movement onset, which was the moment when the monkey released the hand rest button in order to grasp the object. Again, 500ms before movement start were plotted and $1000 \mathrm{~ms}$ afterwards, to see what happens later in the memory period and during grasp.

In summary, in the PSTHs 500 ms of baseline activity can be seen, followed directly by $1000 \mathrm{~ms}$ of activity when the monkey sees or touched the object. The next $100 \mathrm{~ms}$ are right before the memory period starts, so before the light turns of and while the animal is still moving back to the hand rest position, followed by the first 500ms of the memory period, where the animal is not actively doing anything but instead has to memorise the object and wait for the Go cue. The next 500ms are the last $500 \mathrm{~ms}$ before movement starts, followed by $1000 \mathrm{~ms}$ of activity during movement.

Visual and tactile trial conditions are plotted by either solid (visual) or dashed (tactile) lines. Furthermore six different colours were used, each corresponds to one object.

For the passive stimulation task, an ongoing time window, starting $1000 \mathrm{~ms}$ before the platform starts moving upwards towards the finger and ending $1500 \mathrm{~ms}$ afterwards. Here, colours correspond to the stimulus plate used, while dashed and solid lines indicate the direction of movement (solid for movements from left to right and dashed for movements 


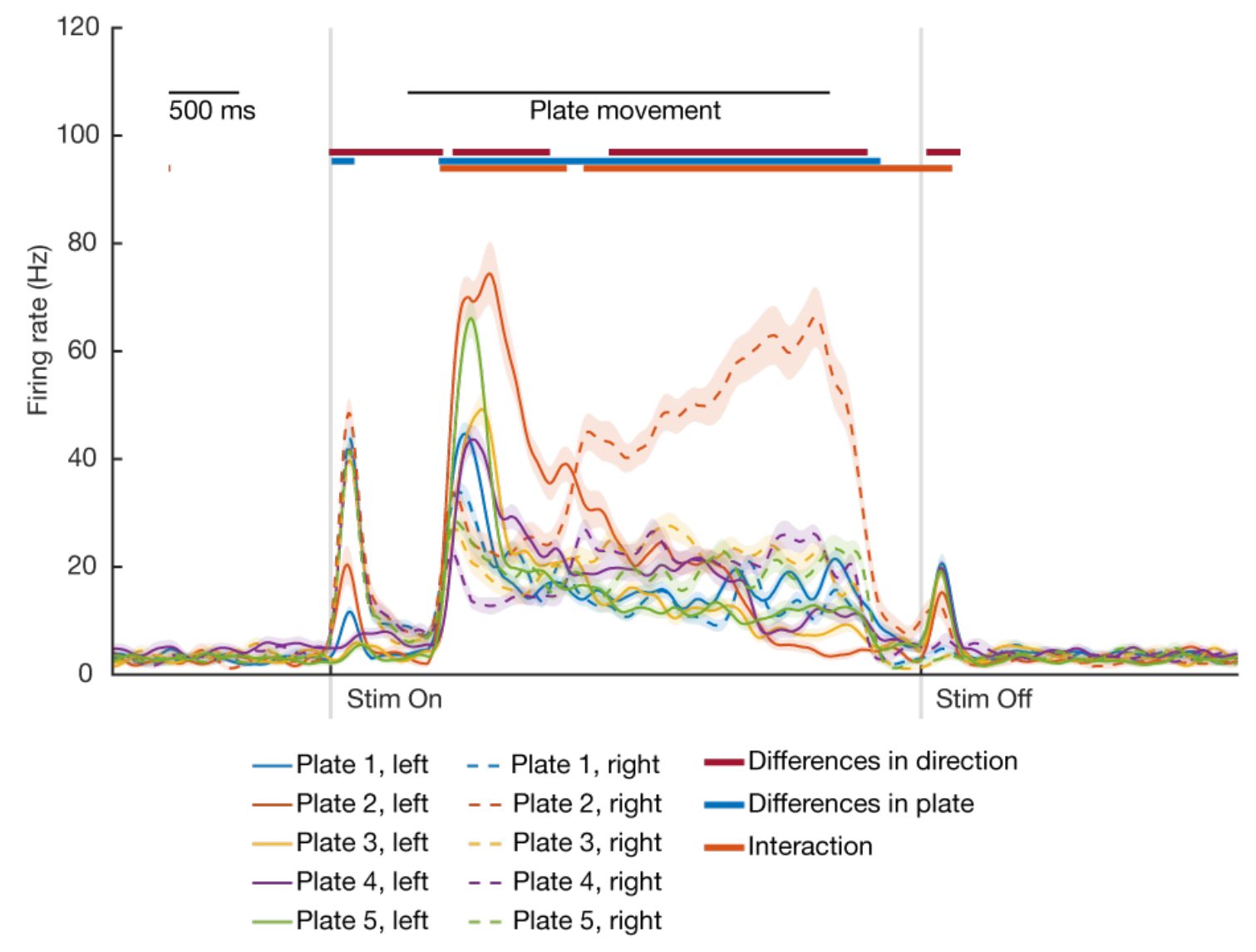

Figure 2.21: Example PSTH of a multi unit in primary somatosensory cortex during the passive stimulation task. This plot is an example PSTH of a unit in primary somatosensory cortex during the passive stimulation task. Plotted is the firing rate in $\mathrm{Hz}$ over time. Each colour corresponds to one plate, while solid and dashed lines mark movement from left to right ("left") or right to left ("right"), respectively.

from right to left). Vertical lines indicate the moment the stimulus touched the finger (stim on) or lost contact (stim off). After end of stimulation 1500ms are plotted. Each plate is marked by a different colour, dashed and solid lines indicate movement direction. Bars on top of the graph indicate significant differences for movement direction, between types or for interaction between the two.

For both experiments, a 2-way ANOVA with Bonferroni correction (over time points) was applied to the data to indicate significant differences between movement direction of the stimulus or stimulus structures (passive stimulation task) or sensory condition or objects (active grasping task), respectively. Bars above the PSTHs indicate significant differences. The shaded area around the curves indicates variance. 


\subsubsection{Population tuning}

To further investigate whether visual and tactile trials result in different brain activities (such as memory activity during memory period), analysis of variance (ANOVA) was used. ANOVA analyses differences between the mean of groups of data points. Here, I used one-way sliding ANOVA for differences between objects and sensory conditions, respectively, with a bin size of $100 \mathrm{~ms}$. Afterwards, a Bonferroni correction for multiple comparison was applied, where the number of comparisons is taken into account to adjust the $\mathrm{p}$-value defined as "significant difference" to a more appropriate one.

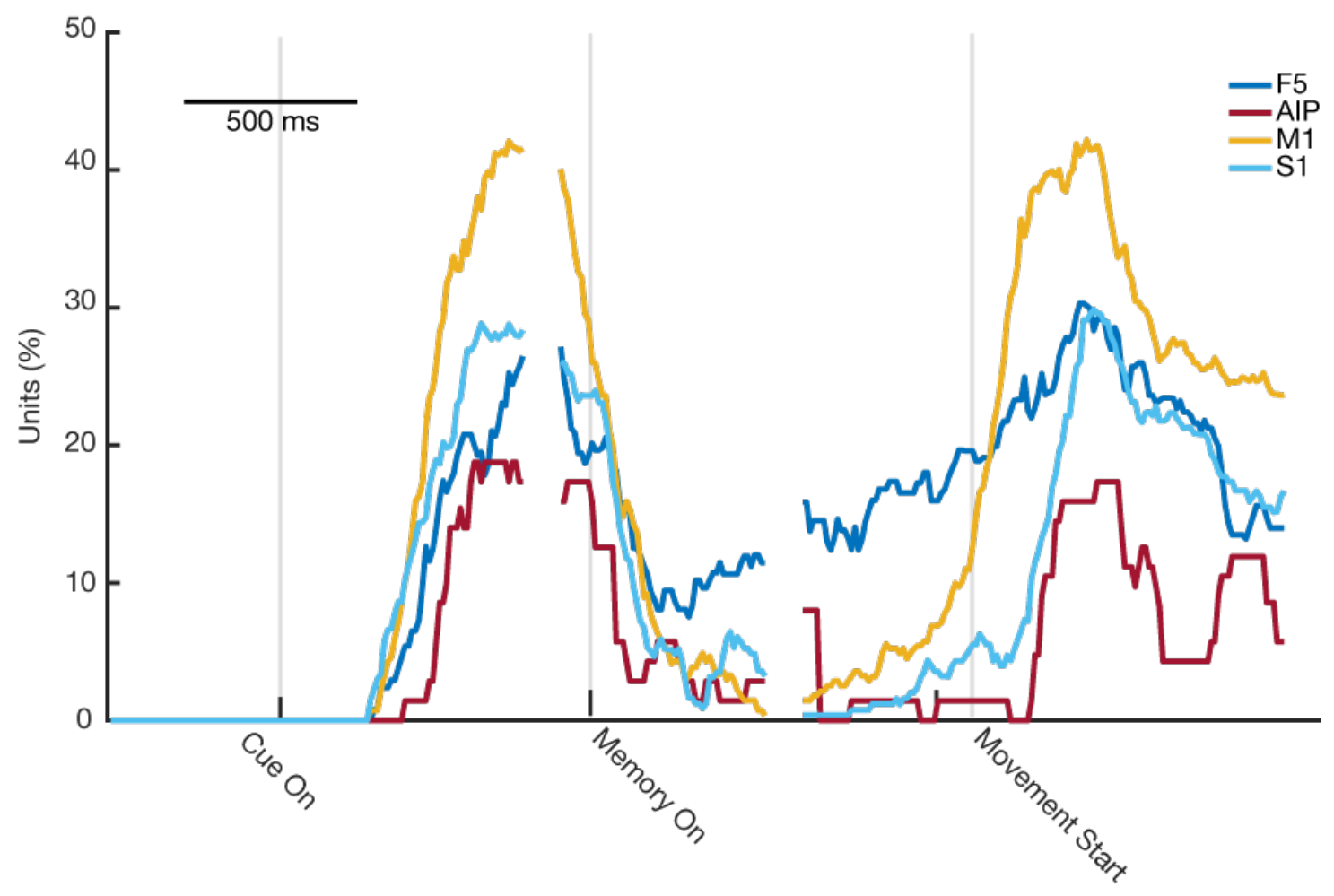

Figure 2.22: Example population tuning analysis for all areas. Example population tuning curve, showing how many units are significantly tuned during each time point of the task in percent. Each colour corresponds to one area, this plot shows how many units are tuned differently for all objects during tactile trials.

To this end, the PSTHs (see 2.7.3) of all neurons were calculated and a sliding one-way or two-way analysis of variance (ANOVA) was applied for each neuron and averaged per area. Afterwards, a one-way sliding ANOVA (comparing task types, visual versus tactile) was applied to the data. The resulting percentage gives information about how many units were significantly tuned towards the investigated condition, with a higher value meaning more units were tuned and therefore showed different activity during all conditions. The percentage of tuned units ( $y$-axis) was then plotted over time ( $x$-axis) and, similar to the PSTHs realigned at points where the task epochs varied in time, to realign the time points to a common point in time (such as appearance of cue information, start 
of memory period and start of movement).

\subsubsection{Receiver operating characteristic}

In order to classify how well single neurons were tuned towards visual or tactile tasks, receiver operating characteristic (ROC) analysis was applied (Dayan, Abbott, et al. 2003). This analysis tries to discriminate between two conditions (in this case visual and tactile trials) and returns a curve where sensitivity (or true-positive rate) and false-negative-rate are plotted against each other. By simply calculating the area below the curve, a direct measure for classification can be found. This measure will be referred to as ROC score in this thesis. The closer the ROC score number approaches one, the better both conditions can be discriminated from each other, while a score of 0.5 would mean chance level.

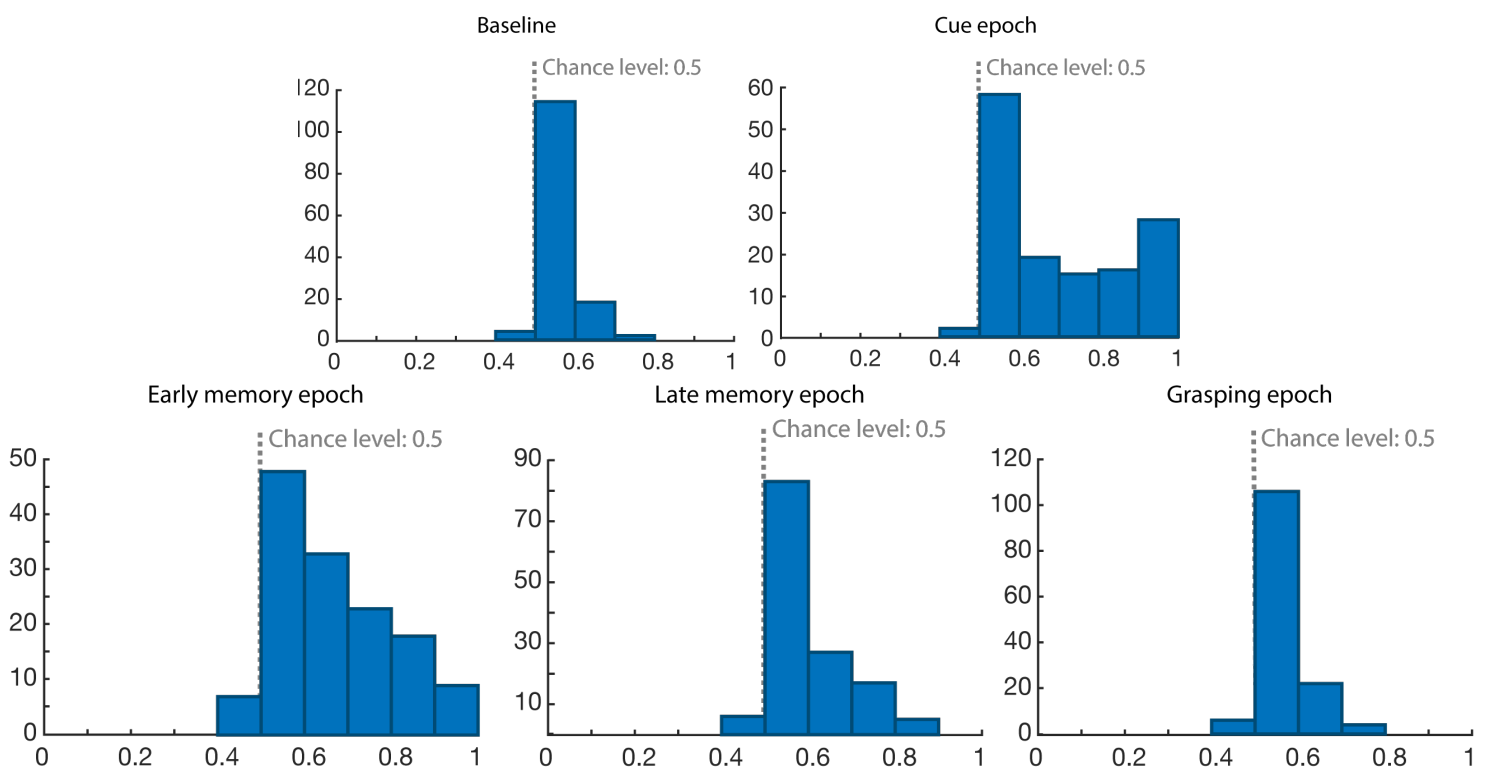

Figure 2.23: Example distribution of ROC scores in one area during the active grasping task. This histogram shows the distribution of ROC scores for different epochs for one example area, in bins of 0.1. The first row shows baseline and cue period, the lower three refer to early memory period, late memory period and grasping.

This was done for all neurons over all sessions separately for five different time epochs. Due to the low number of units in some areas, ROC scores were pooled over all sessions. While this might lead to an overrepresentation of single units (assuming it was present in multiple sessions) the higher $\mathrm{n}$ resulted in overall more reliable results, especially in area AIP, where only between 5 and 16 units could be recorded in each session. Furthermore, ROC score distributions for individual sessions were compared to the pooled distributions (not presented here) to control that the pooling did not result in any unusual patterns. As a control for the measurement, the baseline activity and the first $500 \mathrm{~ms}$ of the cue 
period were analysed. Furthermore the early memory period (500ms after start of memory period), late memory period (500ms before movement start) and grasping period (500ms after movement start) were analysed. Then a histogram of all values (in bins of 0.1) for all neurons per area is plotted, to see how many neurons can discriminate how well between visual and tactile trials at each evaluated time point. An example is given in figure 2.23. Each plot corresponds to one time epoch.

\subsubsection{Decoding}

Decoding was used as an offline analysis to measure how well a decoder can classify the different conditions of the task, based on average firing rate over time. Linear discriminant analysis (LDA) was chosen as a decoder. This classifier attempts to find common features across trials in order to decide which condition a trial belongs to. The classifier tries to reduce the dimension of the data as much as possible while also keeping as much information as possible. The performance of the classifier was evaluated by Leave-

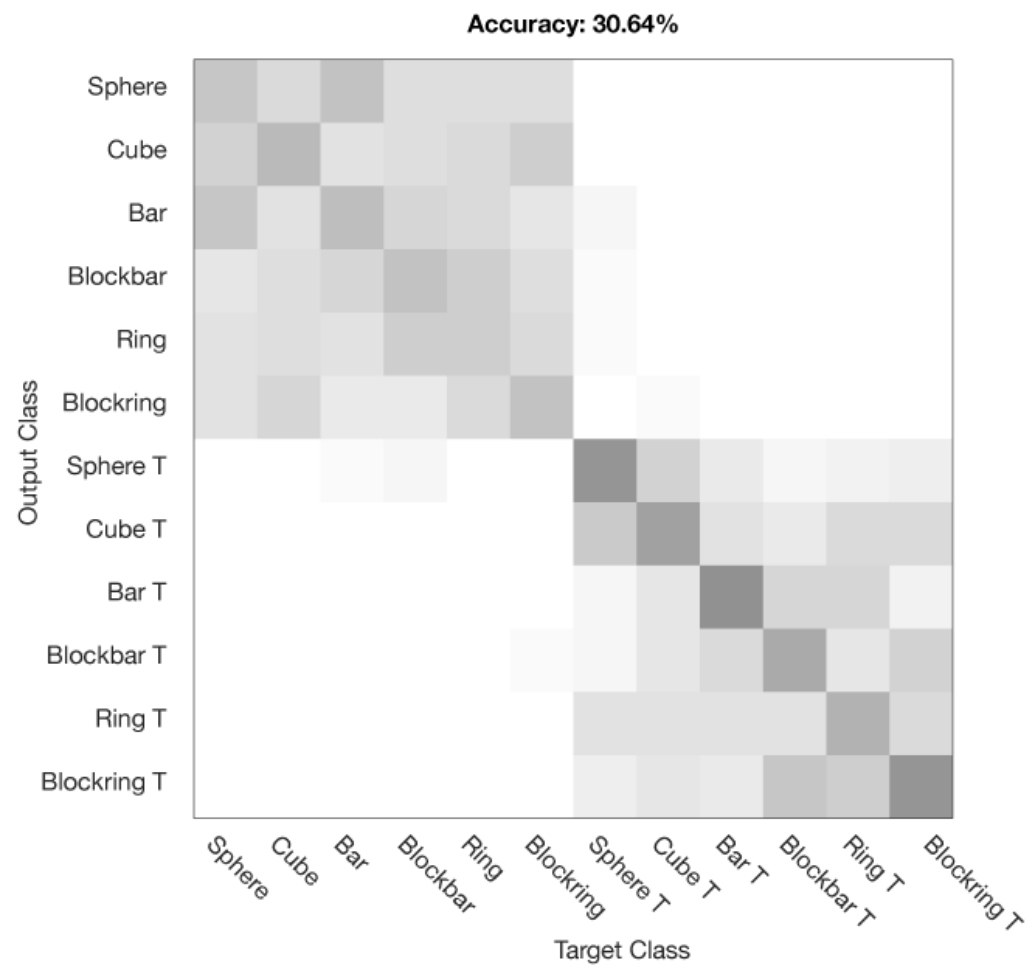

Figure 2.24: One example confusion matrix for decoding of active grasping trials. One confusion matrix, showing how well the LDA decoder predicts each object. Each field corresponds to a pair of a predicted object and the actual object in that trial, showing first all six objects visually presented, then all six objects tactually presented. Darker shades indicate a higher prediction rate. Above the matrix, the overall accuracy is listed.

One-Out cross validation, which means the decoder was trained on all trials but one. Afterwards the decoder needed to predict which condition this left out trial belonged to. 


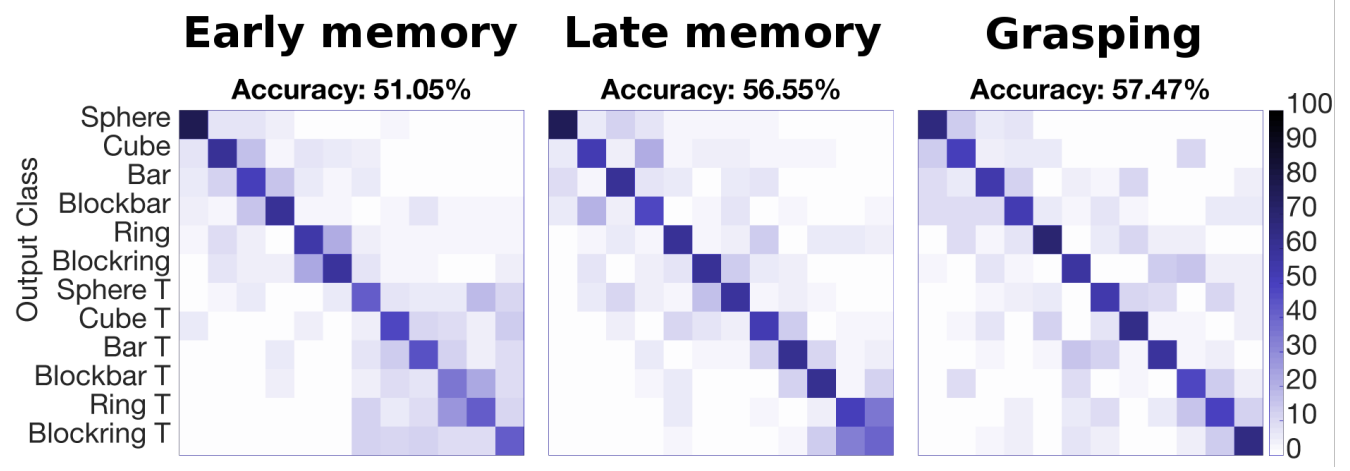

Figure 2.25: Example confusion matrices for decoding of active grasping trials in three different epochs. Three confusion matrices, showing how well the LDA decoder predicts each object. Each field corresponds to a pair of a predicted object and the actual object in that trial. Darker shades indicate a higher prediction rate. From left to right the matrices correspond to early memory, late memory and grasping period.

Since the correct condition was known for all trials, an error rate could be calculated by repeating the Leave-One-Out cross-validation for all trials. In order to illustrate decoder performance, first a confusion matrix is given for three epochs of each area (early memory, late memory and grasping). The mean of 500ms of each period was used for decoding. All objects are listed on the $y$ and $x$ axis, starting with the six objects under visual condition, followed by tactile condition. Darker shades indicate a higher prediction rate. An example of a single decoding confusion matrix can be seen in figure 2.24 whereas figure 2.25 shows an example where all three periods are plotted side to side.

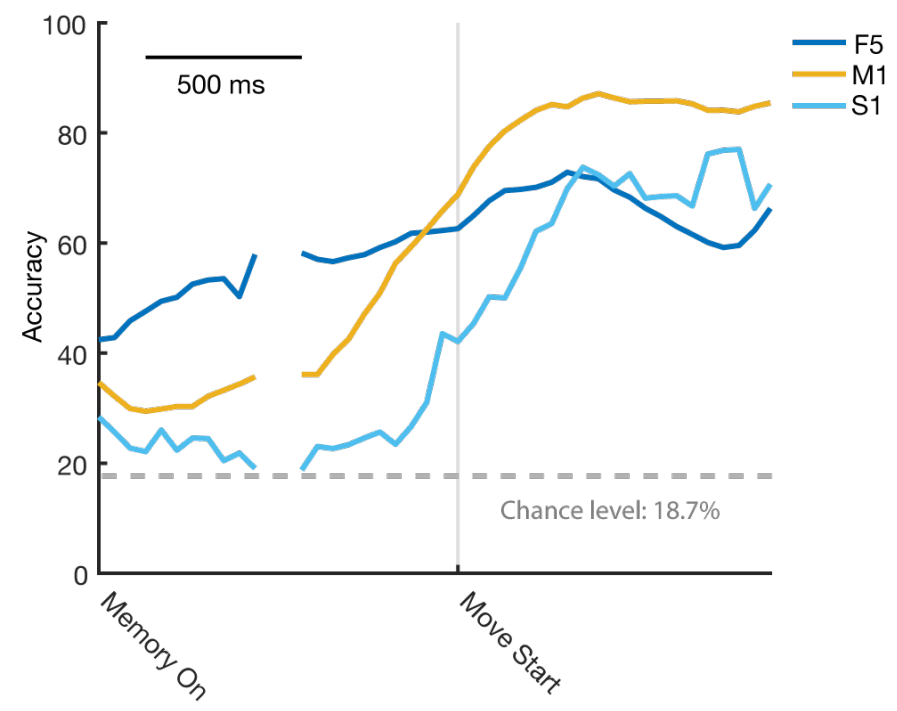

Figure 2.26: Example decoding accuracy over time. This plot shows the accuracy of a decoder over time (averaged over five sessions), starting at the start of the memory period and ending after movement started, for four different brain areas. Each area corresponds to one colour.

A 100 ms window was used for decoding, where always $100 \mathrm{~ms}$ were pooled together 
and the mean of this window was used for decoding. Decoding started in early memory, continued during late memory and ended during grasping period. Performance was then plotted over time, see figure 2.26 for an example. 


\section{Chapter 3}

\section{Results}

In this next section, results for the different analyses described in chapter 2 are presented. First, results for the visual and tactile object recognition task are presented (section 4.1), starting with an evaluation of movement and reaction times of the animal (section 3.3.1) and continuing with analyses that looked first at the activity patterns of a few example units (section 3.3.2) and afterwards whole populations (section 2.7.4). Furthermore, efforts to use the neural activity to predict objects and sensory conditions are presented (sections 2.7.5 and 2.7.6), used as a measure how neuronal activity differs between conditions. Afterwards, results for the passive stimulation task (section 3.4) are discussed, going into activity patterns (section 3.4.1) and population tuning curves (section 3.4.2).

\subsection{Number of units per recording day}

The following table gives an overview over the number of units per recording day per area. After the raw data was spike sorted as described in section 2.7.1, the number of

Table 3.1: Number of units with a firing rate higher than $1 \mathrm{~Hz}$ per area and recording day (active grasping task)

\begin{tabular}{|c|c|c|c|c|}
\hline Recording day & \#units F5 & \#units AIP & \#units M1 & \#units S1 \\
\hline \hline 18.05 .2018 & 36 & 5 & 88 & 60 \\
\hline 01.08 .2018 & 26 & 7 & 62 & 50 \\
\hline 02.08 .2018 & 27 & 6 & 54 & 46 \\
\hline 07.08 .2018 & 25 & 14 & 60 & 53 \\
\hline 09.08 .2018 & 24 & 14 & 55 & 52 \\
\hline \hline 03.01 .2018 & 25 & 16 & 75 & 30 \\
\hline 24.09 .2018 & 24 & 3 & 42 & 38 \\
\hline
\end{tabular}

resulting units with a firing rate higher than $1 \mathrm{~Hz}$ were counted. The data from the pas- 
sive stimulation experiment was filtered but not spike sorted. A lower number of units is therefore not unexpected, since here all signals from a single electrodes were pooled as described in section 2.7.1. Therefore whenever an electrode picked up signals from more than one neuron, this data was simply pooled, whereas spike sorting might help detecting more units per electrode. The reason for the lack of spike sorting for this data was the discovery that tuning decreased over time and was no longer present in the latest recoding session (see section 3.4.2). While spike sorting can improve the quality of population analyses, due to the higher number of units and a more truthful representation of the activity of single units compared to pooling all signals, it is highly unlikely that the tuning would return only through spike sorting.

The first five days, using a white background, were recording days for the active grasping task (section 2.5), the lower two, using a grey background, were days where the passive stimulation task was recorded (see section 2.6). More recordings were conducted but for this thesis only the best (or most representative recording sessions) are presented. For the active grasping task those are the five sessions with the best task performance and trial numbers, while for the passive stimulation task the two recordings which illustrate the diminishing of the tuning best were chosen.

\subsection{Visual and tactile object recognition}

\subsection{Grasping psychophysics}

As described in section 2.5.3, movements of the animal were tracked with a data glove and some camera recordings for visual evaluation were recorded. Here, an example movement for tactile exploration (with grasp correction) and tactile grasping is shown. Since the screenshots of the video are quite blurry, some hand drawn illustrations, that are based on the observed movements, are also given.

In figure 3.1, two screenshots and three illustrations show how the animal tactually explored an object. Since the monkey was unaware which object was in front of him, the first grasp did not match the object shape well. Therefore he was unable to lift the object up. Still, apparently he touched enough surface area of the object that he could immediately correct his grasp to a more suitable one. The second grasp he executed was more suitable for this object. In this example, he squeezed the ring between his thumb and the side of his finger to lift it up. 

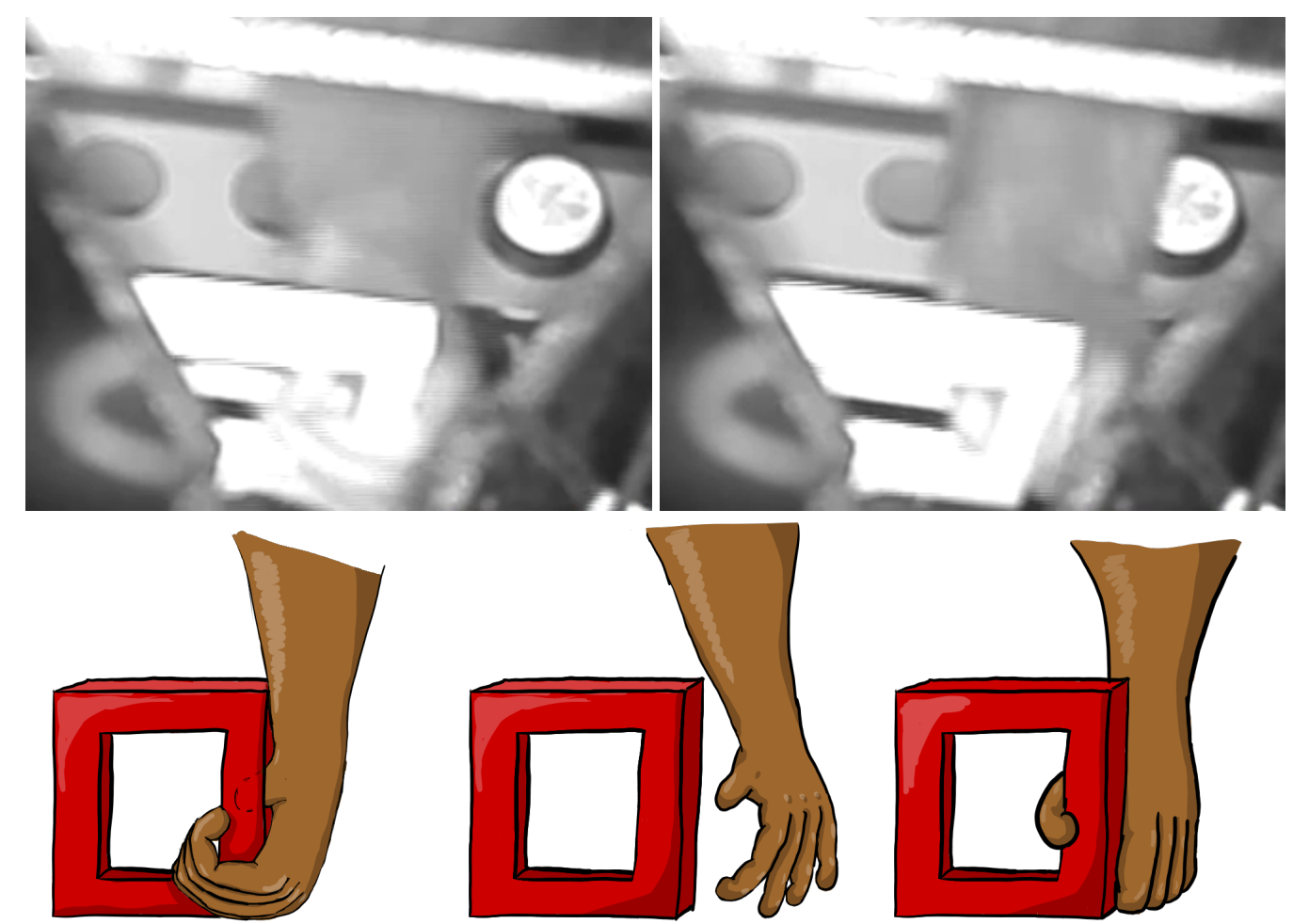

Figure 3.1: Illustration of grasp correction during tactile exploration. Video screenshots and (exaggerated) illustration of the animal correcting his grasp during tactile exploration. Top row shows two screenshots from a trainings session, where the animal first grasped the object in a grasp that made lifting hard. Second image shows the corrected grasp. Bottom row: Illustrations of the same movement. First, the animal touched the object, realized which grasp would be more suitable and corrected its grasp in the second image, leading to the grasp of the third illustration.
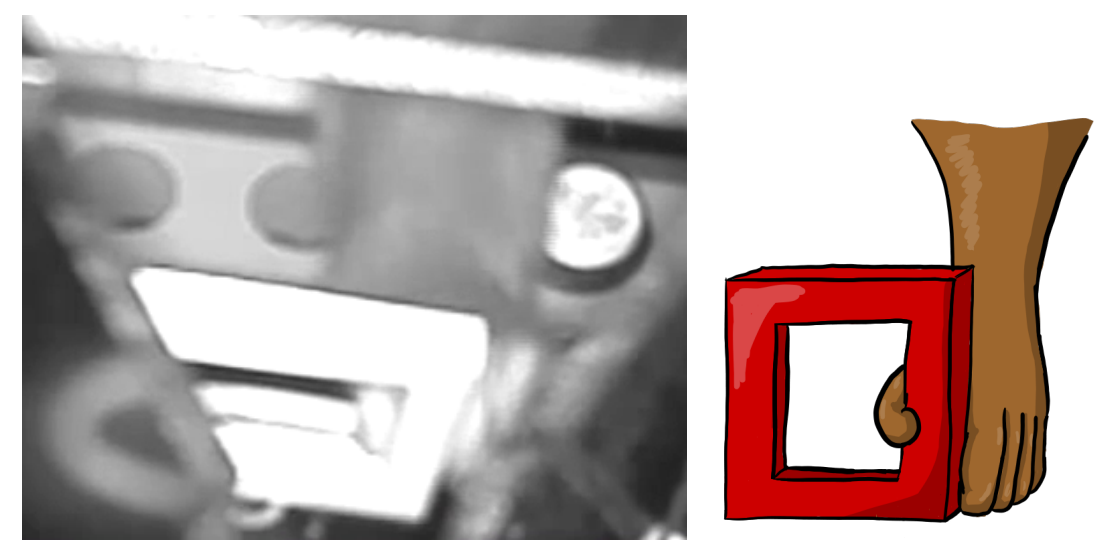

Figure 3.2: Grasp during tactile grasping. Video screenshot and illustration of the animal grasping the object during the grasping period of a tactile trial. Left: Video screenshot of the first grasp the animal used, right: illustration of the same grasp. Compared to tactile exploration (figure 3.1) no correction of the grasp was happening since the first grasp was a suitable one, indicating that the monkey recognized the object.

Figure 3.2 shows the grasping period of a tactile trial. Here, the object identity should be known to the animal, since he had time to tactually explore the object during tactile 
cue (or exploration) phase. Indeed, here no correction motion could be seen. The animal directly squeezed the object between his thumb and the side of his index finger to get a firm grasp and lifted the object up.

This behaviour could be observed consistently during all trials. During the tactile cue phase, the animal often bumped into the object or grasped an object in a way that made lifting it up hard, while he demonstrated that he remembered the object well by directly lifting it during the grasping period, without bumping into it or having to correct his grasp. The latter was true for visual and tactile trials, meaning that seeing and tactually exploring the object both helped the animal to recognise the object.

\subsubsection{Movement and reaction times for grasps during visual and tactile trials were similar while tactile exploration took longer}

As a first control to see whether the monkey used sensory information from the cue phase reaction and movement times were compared, as described in section 2.7.2. Reaction time was defined as the time that passed after the go cue turned off (instructing the animal to grasp) and the moment the handrest button was released. Movement time was defined as the time between the appearance of the go cue and lifting the object completely up.

In figure 3.3 the reaction time of the animal is compared during visual and tactile grasping (during grasping epoch) and tactile exploration of the object during the cue epoch. All trials of all recording sessions were pooled for this analysis.

Panel one ("visual grasping") shows the reaction time the animal needed to initiate movement in visual trials, during the grasp epoch. Values range from $171 \mathrm{~ms}$ to $431 \mathrm{~ms}$ for successful trials, with a mean reaction time of $259 \mathrm{~ms}$. For tactile exploration, the animal needed between $132 \mathrm{~ms}$ and $2674 \mathrm{~ms}$, mean $309 \mathrm{~ms}$. Overall, the resulting curve is a lot wider and has a less pronounced peak, compared to visual grasping. For easier readability, values over $500 \mathrm{~ms}$ were cut off to make the $x$ axis of all three plots the same length. In 61 out of 1261 trials the animal took longer than 500ms to start moving. During grasp epoch, the reaction times for tactually guided grasps looked similar to the reaction times of visually guided trials. Here, the monkey needed between $72 \mathrm{~ms}$ and $464 \mathrm{~ms}$ to start moving. Only in two trials, the reaction time was faster than $180 \mathrm{~ms}$ (both times $72 \mathrm{~ms}$ ). While different time epochs (such as the memory period) had a random length in a certain range to make it impossible for the animal to guess the appearance of the go cue, this fast reaction time could indicate successful guessing of the appearance of the go cue in these two trials. As can be seen by the other values, a reaction time above $130 \mathrm{~ms}$ would be expected if the animal actually waited for the go cue to disappear and initiated 
movement only after being instructed to do so.

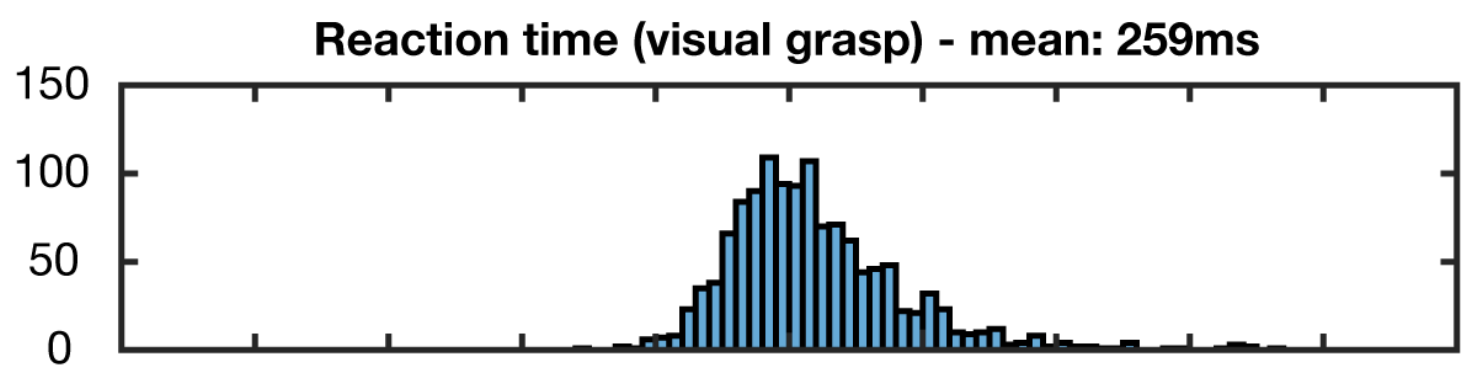

Reaction time (tactile exploration) - mean: 309ms

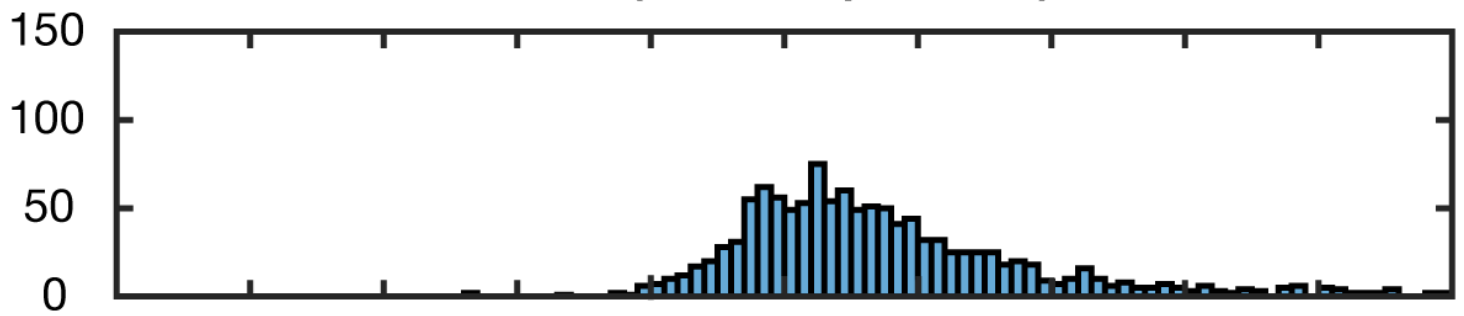

Reaction time (tactile grasp) - mean: 256ms

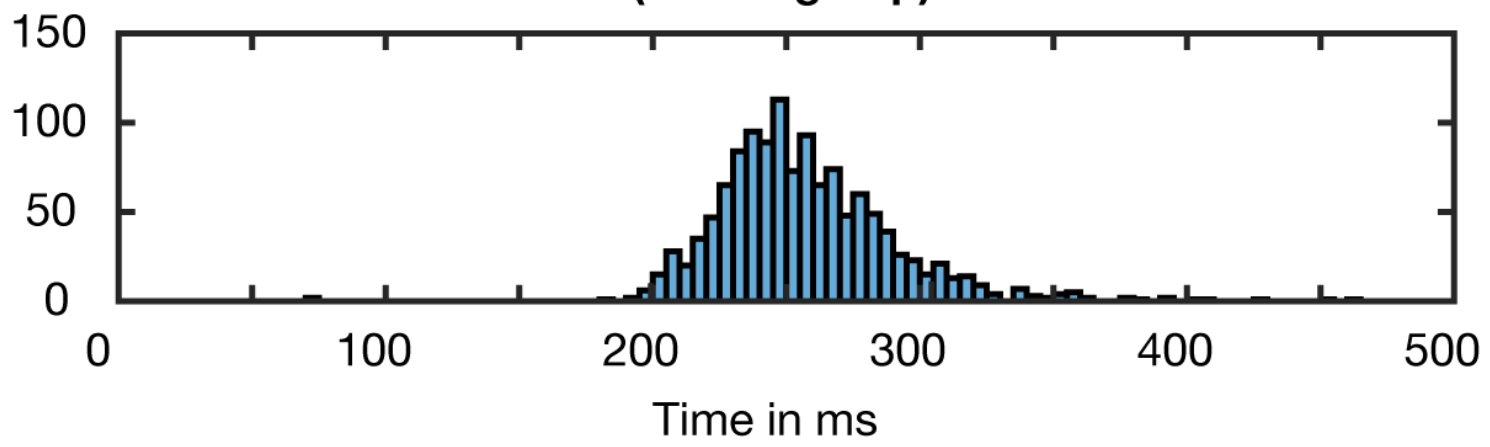

Figure 3.3: Reaction times during active grasping task. A histogram, showing the distribution of reaction times during the active grasping task during visual and tactile exploration and grasp epoch. This histogram illustrates how often certain reaction times occurred during all recording sessions, listing the times in milliseconds. Reaction times during tactile exploration that took longer than $500 \mathrm{~ms}$ were cut off due to better readability $(n=61)$. Reaction times during grasping in visual and tactile trials were quite similar, while they were slower and more widely distributed during tactile exploration.

Figure 3.4 shows the movement times during either visual and tactile grasp or tactile exploration. For visual trials ( $n=1284$ ), the animal needed between $447 \mathrm{~ms}$ and $869 \mathrm{~ms}$ to grasp and lift the object in front of him, with a peak around 540ms. Interestingly, these numbers were similar during tactile grasping $(n=1261)$, with a range between $441 \mathrm{~ms}$ and $835 \mathrm{~ms}$ and a peak around $550 \mathrm{~ms}$. The histogram for tactile exploration however is far more spread out. First of all, the animal was given more time during tactile exploration, to ensure that he was able to identify the object without timing out. During the grasp epoch, all trials that took longer than $870 \mathrm{~ms}$ were marked as wrong to encourage the animal to use the cue information and avoid fiddling around too much. For tactile exploration, the 
animal was given $3000 \mathrm{~ms}$, the actual movement time he needed to tactually explore and lift the object fell between 557 and $2980 \mathrm{~ms}$, where he took longer than $870 \mathrm{~ms}$ in 372 of 1261 trials. Furthermore, there was no clear peak for the time the animal needed to complete tactile exploration and lifting the object, as the values are distributed more evenly. Movement during tactile exploration that took longer than $1400 \mathrm{~ms}$ were cut of (55 trials) for better readability.
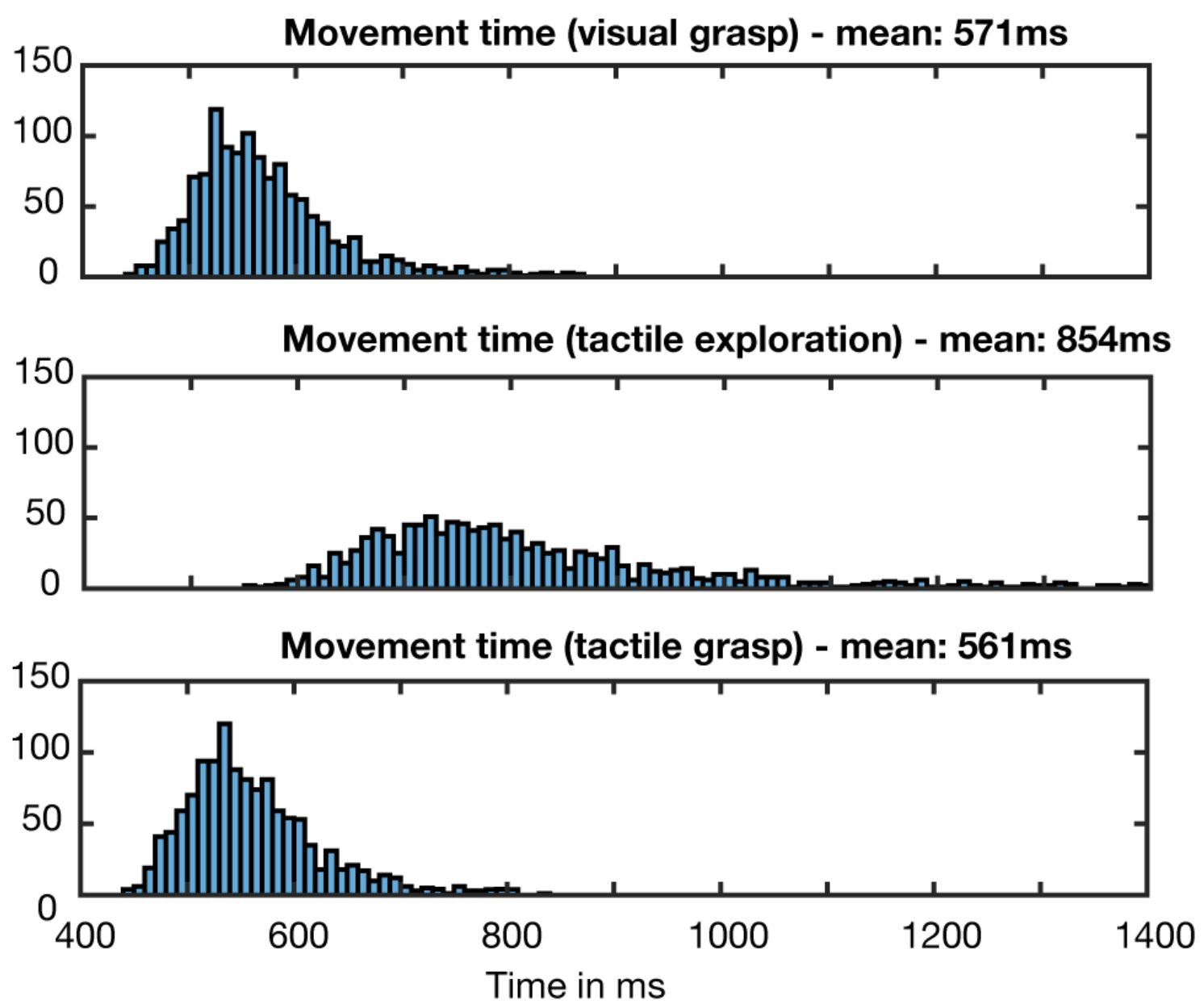

Figure 3.4: Movement times during active grasping task. Movement times for visual and tactile grasping and tactile exploration. This histogram lists how often certain movement times occurred during all recording sessions, listing the movement times in milliseconds. Movement during tactile exploration that took longer than $1400 \mathrm{~ms}$ were cut off (55 trials) for better readability. Movement times during grasping epochs in visual and tactile trials were quite similar, whereas they were more widely distributed during tactile exploration.

Comparison of video recordings and hand kinematics (section 2.5.3) confirm that the animal performed a more straightforward movement when he had to grasp an object after seeing or touching it, compared to grasping an unknown object during tactile exploration (see section 3.3). During tactile exploration, the animal often bumped into the object at a less optimal angle that did not allow him to grasp the object right away, and he had to adjust his hand position and shape, which resulted in longer movement times. 


\subsubsection{Unit activity varied per area}

After recording data from 256 electrodes over five recording sessions, I started to analyse single neuron activity (average firing rate over time) in all four brain areas as a first measure of selectivity. First, all data was spike sorted as described in section 2.7.1 and afterwards all channels with a firing rate higher than $1 \mathrm{~Hz}$ were plotted as $\mathrm{PSTH}$, as described in section 2.7.3. Afterwards a sliding two-way ANOVA was applied to see significant differences between objects, sensory condition or an interaction between both, indicated by lines above the plot. Due to the size of this data set, only a few example units per area will be presented here.

\subsubsection{Strong motor activity in area F5}

In this section, two units recorded from the ventral premotor area F5 are presented.

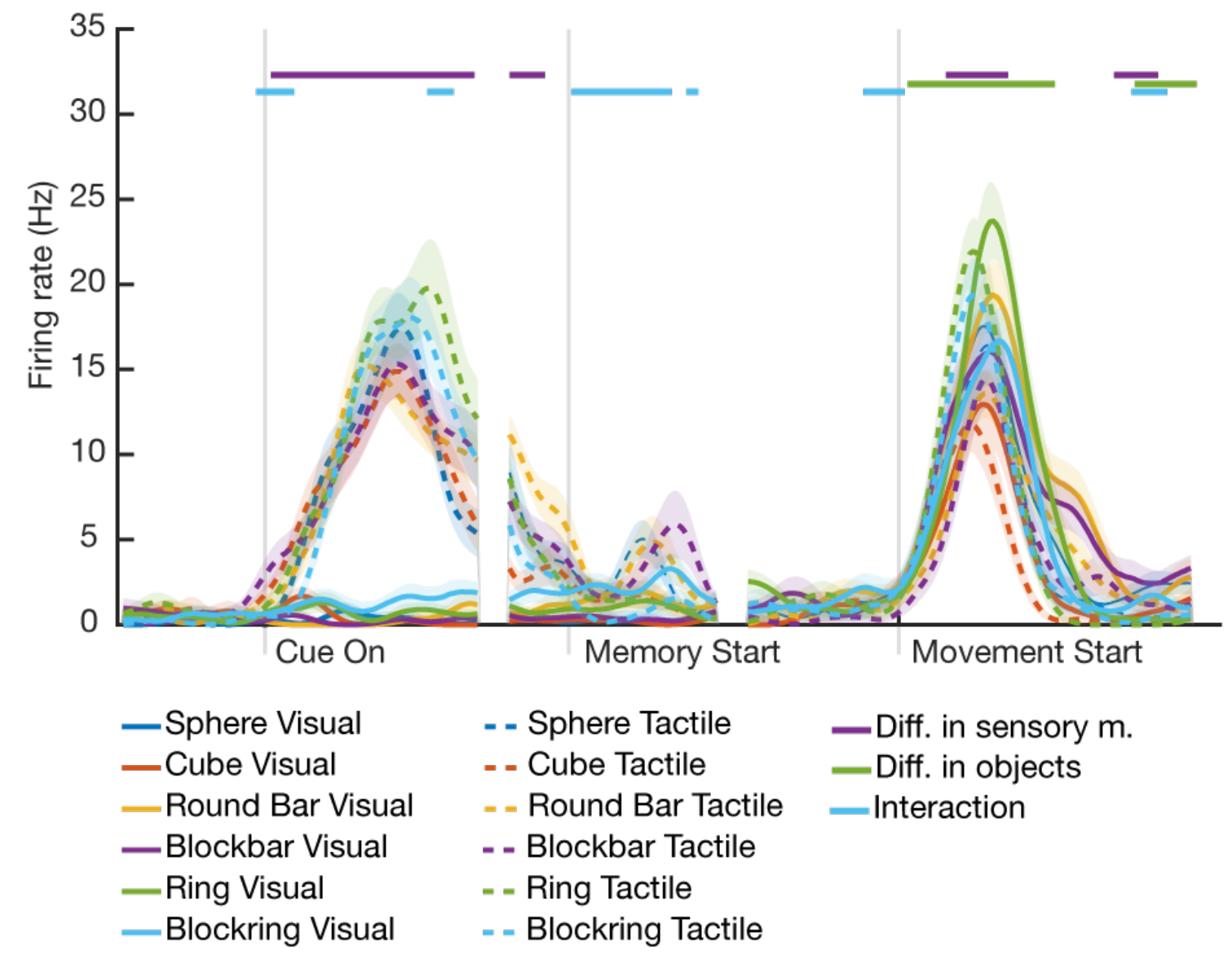

Figure 3.5: Firing rate of an F5 unit, reacting to movements. One example unit from area $\mathrm{F} 5$, showing the firing rate in $\mathrm{Hz}$ over time, realigned after cue presentation and start of memory period. Here, an increase in activity during movement of the animal (either tactile exploration or grasping of the object) can be seen as well as a small increase during the early memory epoch. 
During all five recording sessions, between 24 and 36 units were recorded and could be separated during spike sorting (see section 3.1 for the number of units during each recording session). Different colours mark the different objects, while dashed and solid lines are used for tactile and visual trials, respectively.

The first example unit (figure 3.5) was mainly active during movements of the animal, such as tactile exploration of the object during the tactile cue phase and when lifting the object during the grasp period, where an significant increase in activity can be seen. During illumination of the object, the unit activity stayed at baseline activity (first $500 \mathrm{~ms}$ of the plot). Interestingly, activity did not differ for different objects during tactile exploration, even though the movement of the animal varies to some degree, since some objects required a different hand shape. During memory, the activity of this unit showed a small increase in activity in tactile trials, before returning to baseline. Interestingly, activity during grasping epoch is similar between both sensory conditions but different for the objects (ranging between $12 \%$ and $23 \%$ ).

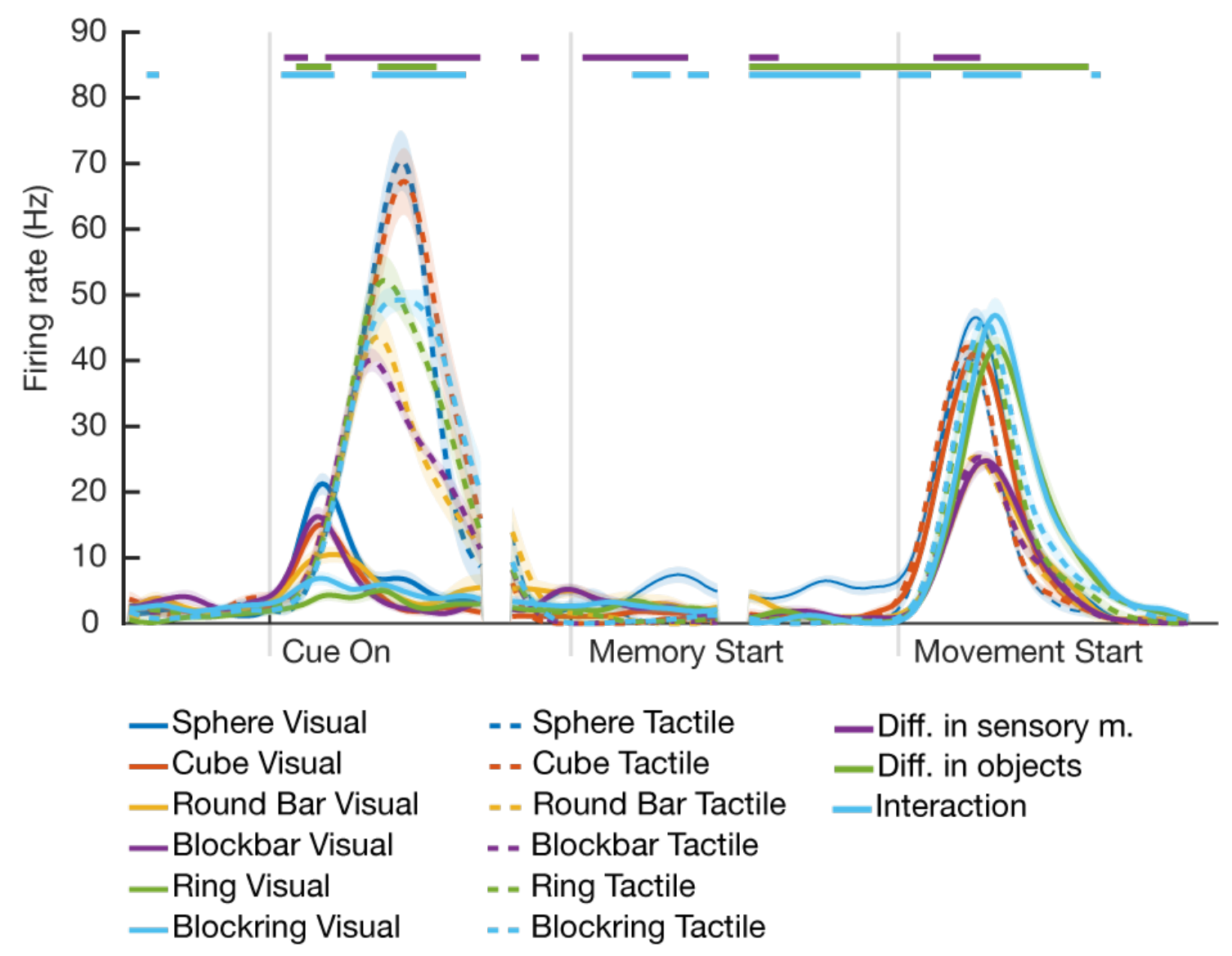

Figure 3.6: Firing rate of an $\mathbf{F} 5$ unit showing some additional activity increase during visual cue. A second example unit from area F5, showing firing rates over time in $\mathrm{Hz}$, realigned at cue presentation and start of memory period. Again, an increase in activity during movements can be seen. This unit also showed a small increase in activity during visual presentation of the cue but no memory activity, where activity was very similar to baseline. 
The second example unit (figure 3.6) also showed firing rate modulation with movement, although here different objects led to a different activity pattern during tactile exploration. This unit also showed a small increase during the illumination of most objects during the visual cue phase of some objects. This unit did not show any memory activity. Again, the firing rate during grasp epoch was similar between both sensory conditions but different for objects. Objects that were grasped with the same grip type showed a similar activity pattern, implying similar movements when grasping these.

\subsubsection{Strong motor activity in area M1}

Usually, neurons in motor cortex are associated with movement activity, but activity from single neurons can differ from this expected pattern. Here, three example neurons from the primary motor cortex are shown. Between 54 and 88 units were recorded on each recording day (see table 3.1 for number of units per recording day).

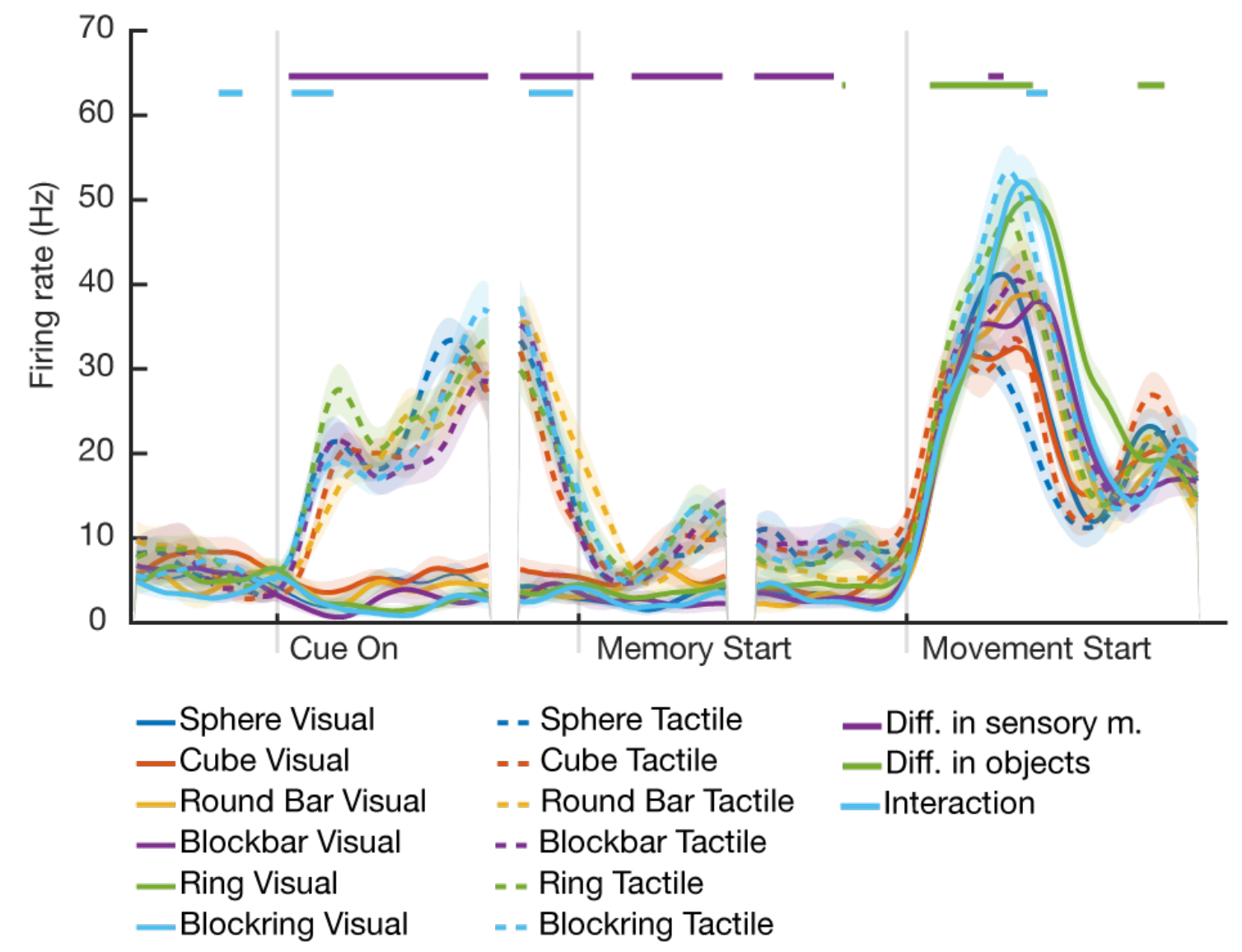

Figure 3.7: Firing rate of an $\mathrm{M} 1$ unit, reacting to movements. An example unit from the primary motor cortex, plotted is activity in $\mathrm{Hz}$ over time. As expected for an unit in motor cortex, a strong modulation in activity with movements of the animals could be seen. This unit also showed a small memory effect for tactile trials during memory period, where activity was increased compared to baseline activity. 
The first unit (figure 3.7) is a very typical unit that increases activity when the animal moves (either to tactually explore or to lift objects) and otherwise shows barely any changes in activity. During cue, a significant difference in tuning between both conditions can be seen. There is a small increase in activity during tactile trials in the memory period, compared to the baseline period (first 500ms of the plot). Again, this increase is significantly different between visual and tactile trials. There is almost no change in activity during visual presentation of the objects in visual trials.

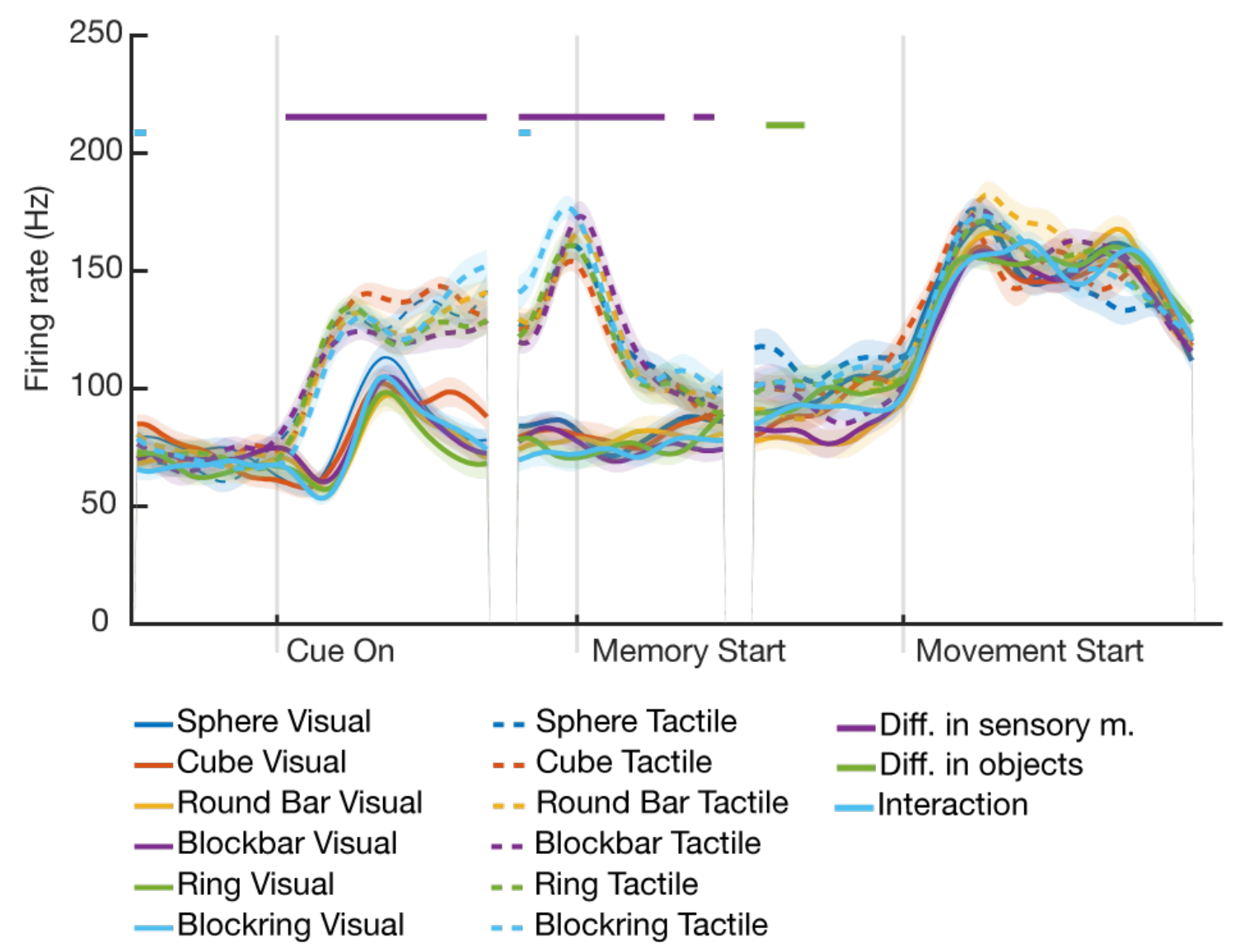

Figure 3.8: Firing rate of an M1 unit showing additional activity increase during visual cue. A second example unit from area M1. This unit showed an increase in activity during movements but also a smaller increase in activity during visual cue presentation and a slightly increased activity during memory period.

The second unit (figure 3.8) showed an increase in activity while the object is seen, while also showing the typical activity increase during movements. Again, a significant difference in tuning can be seen during cue period and early memory period. This effect disappeared during late memory, here an increase in activity could be seen (compared to baseline) but no significant difference between visual and tactile trials or different objects was found. 


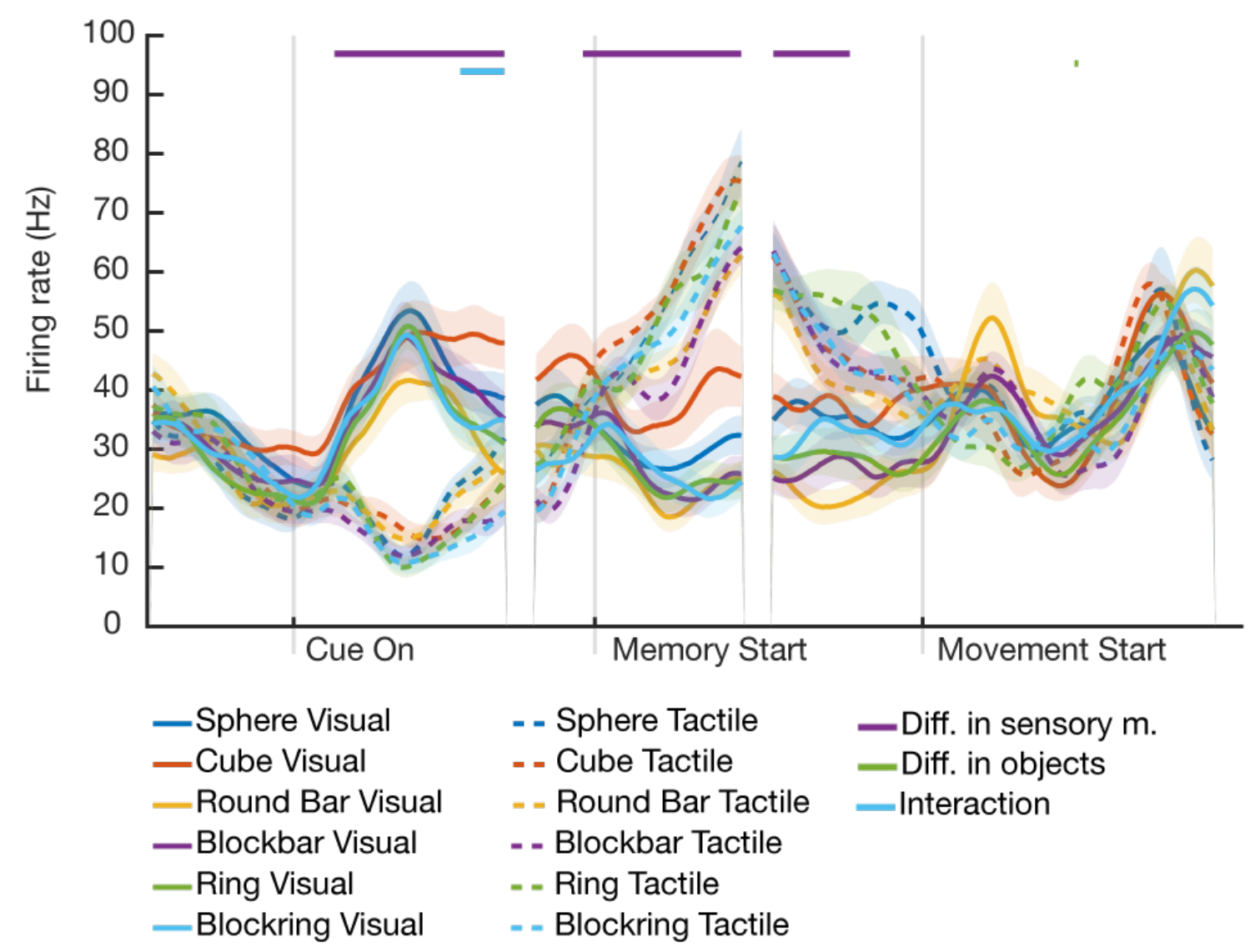

Figure 3.9: Firing rate of an M1 unit showing some unusual activity pattern. A third example unit from area M1. The activity pattern of this unit was more varied, showing an decrease in activity during tactile exploration but an increase during grasping of the object. Activity also increased during visual presentation of the object and memory period, where a sigificant difference between visual and tactile trials could be found.

The third unit (figure 3.9) showed a more complex activity pattern, where the activity actually decreased during tactile exploration but increased shortly before the memory period started and during the memory period. On the other hand, the activity increased during visual presentation of the object, even though no movement of the hand or arm was occurring, only to decrease during memory period. During cue and memory period, a significant difference in tuning between visual and tactile trials could be found. During the movement period, activity changed in a more expected way, correlating with movements independent of the sensory condition after a short drop in activity at the movement start.

During tactile exploration the activity of the first two units was very similar for the first couple hundred ms (the reach component of the task) and then splitted in more different activity patterns during the actual exploration, where the animal moved differently due to the different shapes of the objects. 


\subsubsection{Strong correlation with presence of tactile input in area S1}

The third area in which I analysed the activity of single recorded units is the primary somatosensory cortex. During the five recording days, between 46 and 60 units were recorded and separated during spike sorting on each day (for exact numbers see section 3.1). Two example units from this area are shown below.

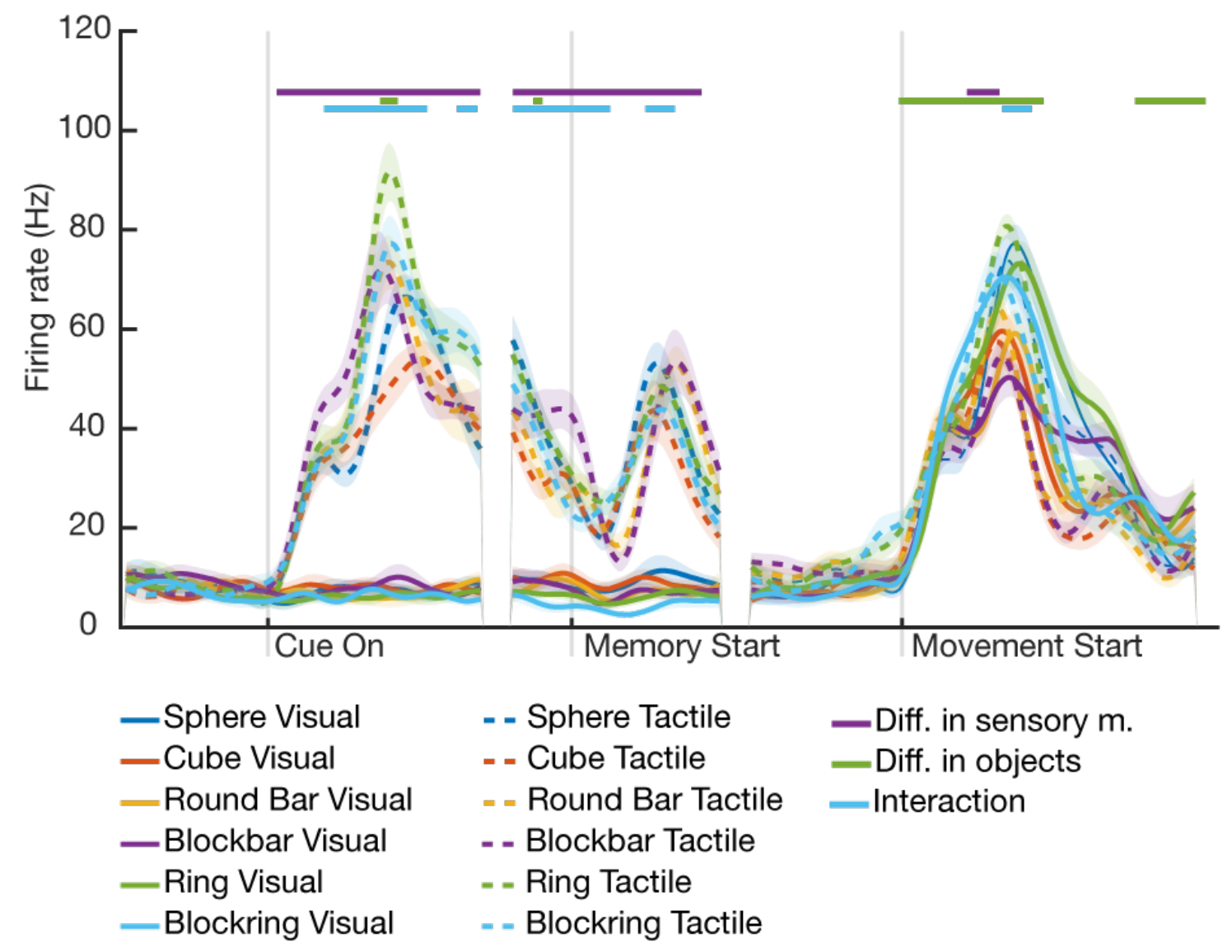

Figure 3.10: Firing rate of an S1 unit, corresponding with tactile input. An example unit from primary somatosensory cortex. This unit was mostly active during time epochs when tactile input was present. Activity was similar during tactile exploration and lifting of the object.

As expected for units in the primary somatosensory cortex, both units showed an increase in firing rate during epochs in which tactile input was present, but not during epochs where visual information was present. During tactile trials, both units (figures 3.10 and 3.11 showed an increase in activity during tactile exploration of the object during the cue epoch and during the grasping of the object (where it was touched and grasped in order to lift it). The first unit (figure 3.10) showed similar activity during tactile exploration and lifting of the object, whereas the second one (figure 3.11) had an increased firing rate during lifting, compared to tactile exploration. Neither unit showed an activity increase during visual presentation of the object (cue epoch of visual trials). Both units showed 
a more or less strong increase in activity during the start of the memory period (shortly after the handrest button was pressed again), which decreased strongly at the end of the memory period, when the activity during most tactile conditions was only slightly increased in comparison to baseline activity.

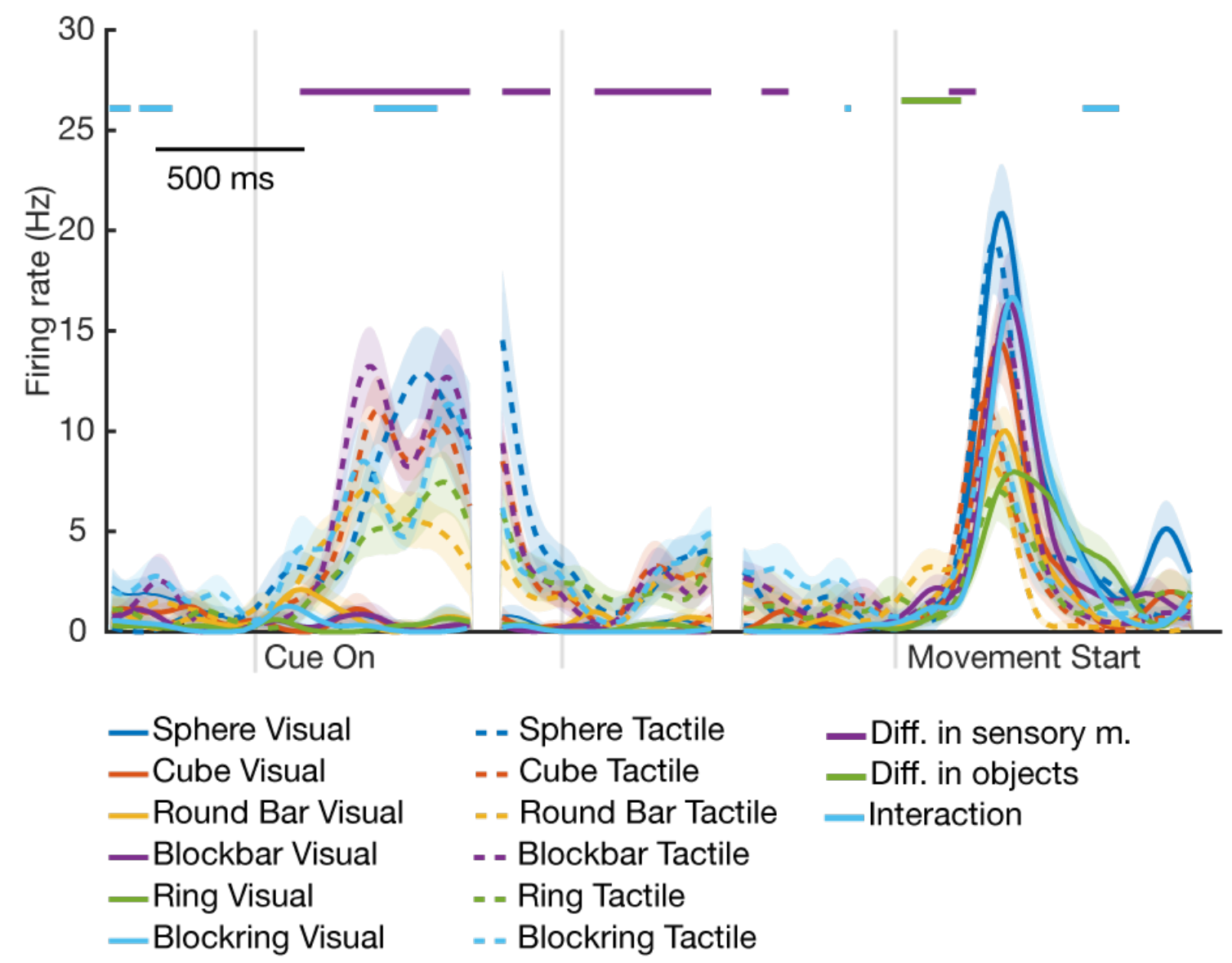

Figure 3.11: Firing rate of an S1 unit, modulated by tactile input. An second example unit from S1. This unit was also mostly active during time epochs when tactile input was present. During tactile trials there was also an increase in activity shortly after memory period started.

\subsubsection{Unit activity in area AIP}

The number of recorded units in the anterior intraparietal cortex was overall very low, as only 5 - 16 units could be recorded during each recording session (for details see table 3.1). Even though this area is mostly known for reacting to visual information about objects that are about to be grasped, very few units showed this behaviour. Firing rate of most units was quite low (especially compared to other areas) and noisy, even after noise reduction and spike sorting, as described in section 2.7.1. Here, one example unit from the anterior intraparietal cortex is discussed, showing less typical behaviour. The example unit (figure 3.12) showed a less expected pattern for an area that is mostly known to re- 
spond to visual properties of objects that are about to be grasped, as no strong increase during visual presentation was observed. On the other hand, the unit increased its firing rate during memory epoch, showing a memory effect that was stronger for tactile than for visual trials. For this unit, it is also important to notice the slow overall firing rate, which rises up to around $8 \mathrm{~Hz}$, a lot lower than firing rates found in other areas. Overall, recording quality in this area was very poor, as noted by the low number of units and a generally low firing rate.

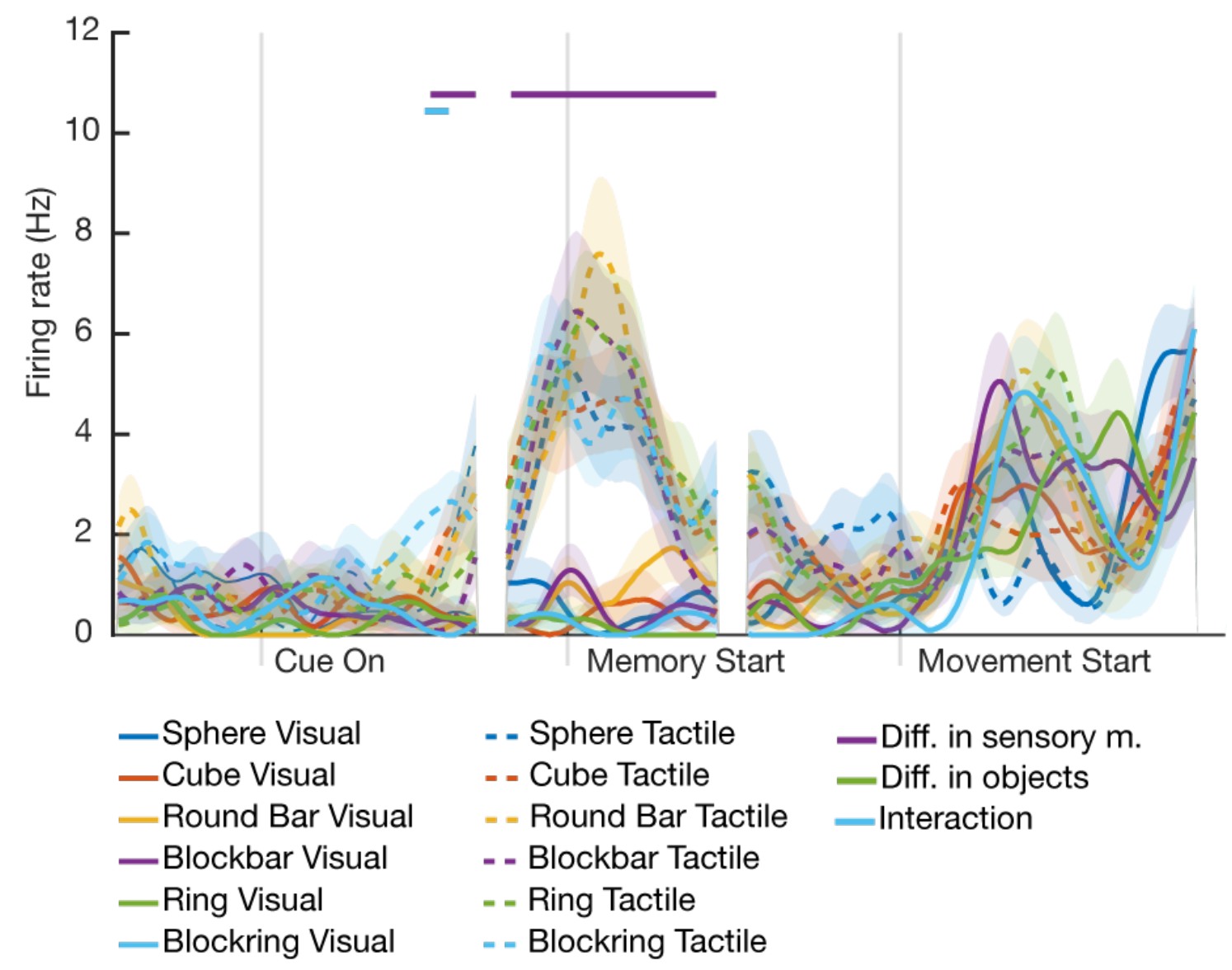

Figure 3.12: Firing rate of an AIP unit, mainly active during tactile exploration and grasping. An example unit from area AIP. This unit was mostly active during memory epoch in tactile trials and shows no activity increase during visual object presentation. Overall, this unit had a very low firing rate (around $8 \mathrm{~Hz}$ maximum) and seems more noisy than the examples from other areas.

\subsubsection{Population tuning revealed differences in activity between visual and tactile trials in the memory epoch}

In addition to analysing the activity of individual units, a population analysis was performed over five recording sessions and averaged afterwards, as described in section 2.7.4. In all three analyses plotted here, a sliding ANOVA (with Bonferroni correction for mul- 
tiple comparisons over time points) was applied to either a set of trials (only visual or tactile) or all trials, to determine whether significant differences exist between objects for each area or sensory conditions.

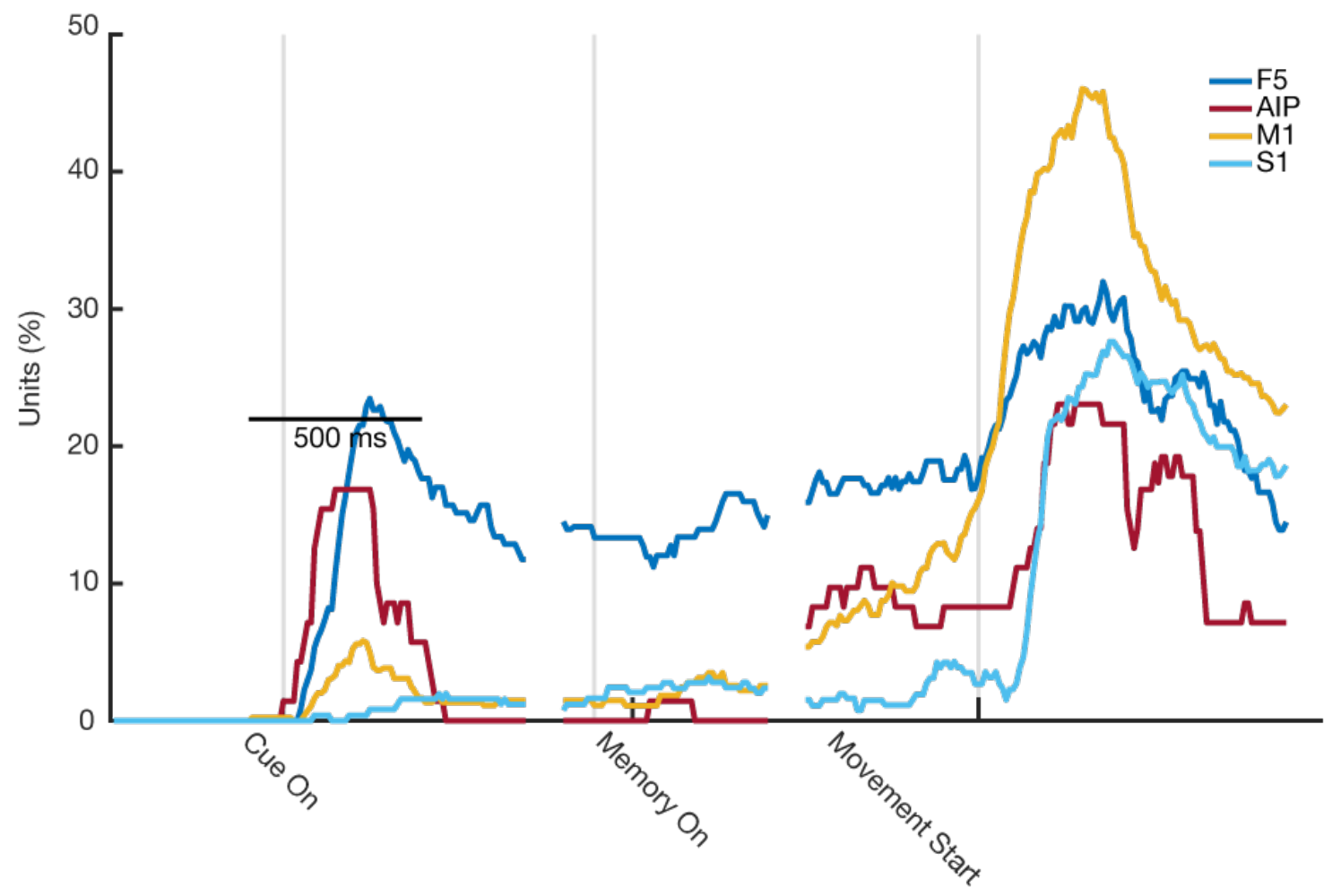

Figure 3.13: Number of significant units at each time point for visual trials during active grasping exeriment. This plot shows the number of significantly tuned units (ANOVA, factor objects) in percent for each time point. F5, AIP and M1 showed an significant difference during cue and all areas show a significant modulation during grasping.

In figure 3.13, the number of significant units at each time point for all visual trials is plotted for areas M1 (yellow), F5 (dark blue), S1 (light blue) and AIP (red). Vertical lines mark the onset of the cue, in this case start of illumination of the object, start of the memory period and movement start. During visual presentation of the objects, up to $23 \%$ of units were selective to the presented objects in premotor area F5 and 17\% in AIP. During memory period, a memory effect could be seen in F5 (but no other area), where again around $17 \%$ of units remained significantly tuned between different objects. When the movement started, the number again increased to $32 \%$ and from there decreased during movement. For primary motor cortex, only around $6 \%$ of units were significantly tuned during the visual cue presentation epoch but tuning decreased as visual presentation continued. During memory, between $4 \%$ of units were significantly tuned during early memory. This number rose as movement start approached. When movement started tuning reached its peak at $46 \%$ during grasp epoch. The population of recorded somatosensory units mainly showed significant tuning during grasp epoch. Between cue presentation (illumination of the object) and end of memory period, only up to $4 \%$ of units were sig- 
nificantly tuned, compared to up to $28 \%$ during grasping (and therefore touching) of the object. AIP also showed a rise in the number of significantly tuned units during grasping, where around $10 \%$ of all recorded units were tuned.

In figure 3.14 the same analysis was performed for tactile trials. While the overall shape was similar after the memory epoch started, some small differences were still visible. However, the tuning during the cue period was different, which is not surprising since the animal was actively moving and touching (without seeing) the object, whereas the animal was doing the opposite during visual trials (seeing the object but not moving). In all three areas, a higher number of units was significantly tuned during the cue epoch, as compared to visual trials. At a first glance, the memory epoch looked similar, although some differences existed. Overall, in F5 less units were tuned during memory (15\% compared to $23 \%$ during visual trials), although a similar steady memory effect was visible. $\mathrm{M} 1$ and $\mathrm{S} 1$ showed very few significantly tuned units during early memory, starting to rise only shortly before the movement started (around the time when the go cue appeared). During grasp, the overall shape and height of the curve was similar between visual and tactile trials. In area AIP, the number of significantly tuned neurons rose during epochs where movement was happening.

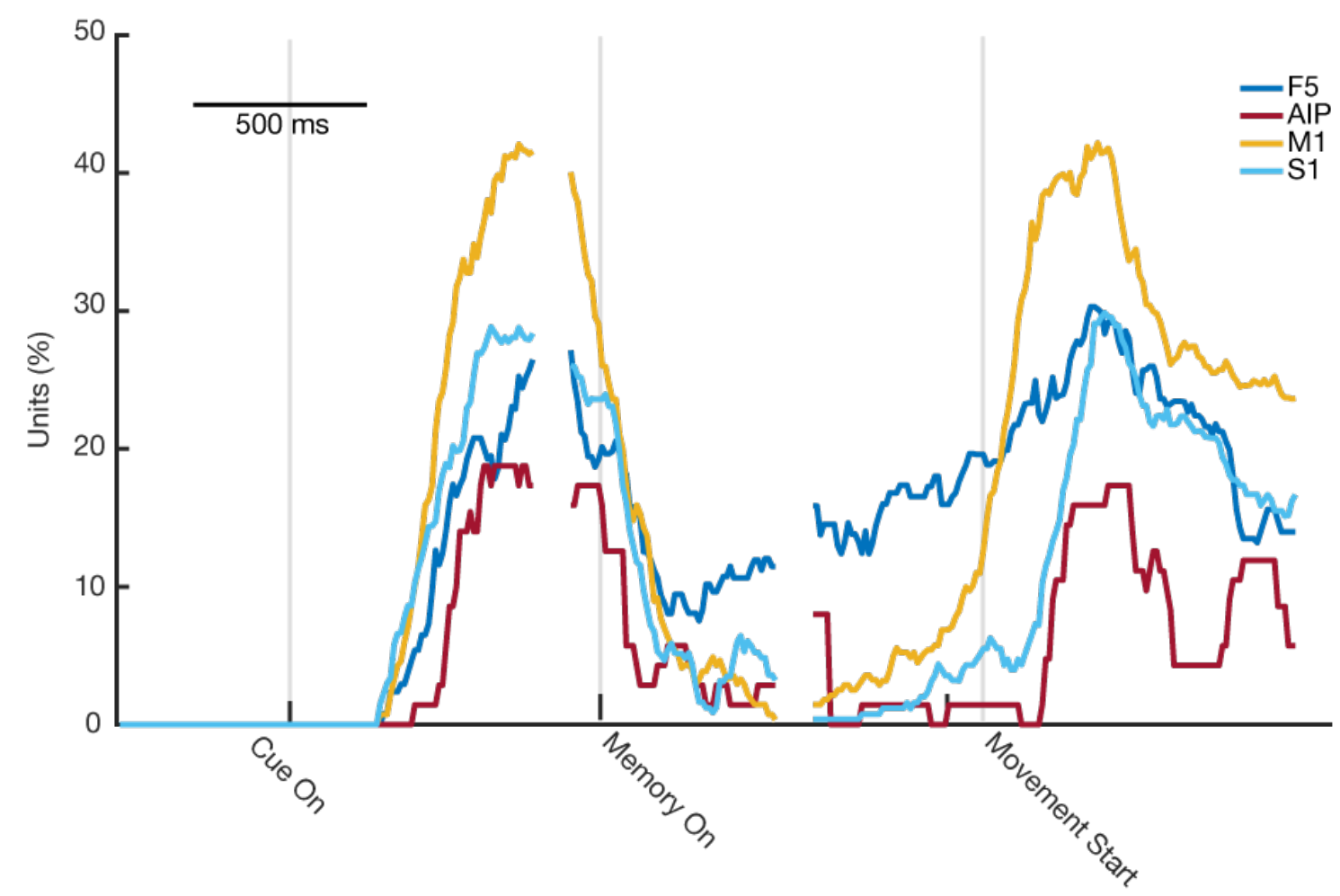

Figure 3.14: Number of significant units at each time point for tactile trials during active grasping task. This plot shows the number of significantly tuned units (ANOVA, factor objects) for each time point of the tactile active grasping task trials for areas F5, M1, AIP and S1. Here, M1, AIP and S1 show tuning towards epochs that contain movements, while F5 also has an increased tuning during memory epoch. M1 has no increased tuning during early but during late memory, shortly before movement starts. 
Finally, in a third analysis, a sliding ANOVA was applied to all trials (visual and tactile ones), using the sensory condition as variable. figure 3.15 shows how many units are significantly tuned towards the sensory conditions (visual cue presentation versus tactile exploration). Interestingly, here some significantly tuned units during baseline could be found with up to $6 \%$ in F5. During cue epoch, a strong and significant difference could be seen in all four areas between visual and tactile conditions, stemming from the two different ways of cue presentation. During early memory, all areas show a significant difference around $50 \%$ (around $30 \%$ for AIP) that decreases dramatically during late memory epoch and grasping.

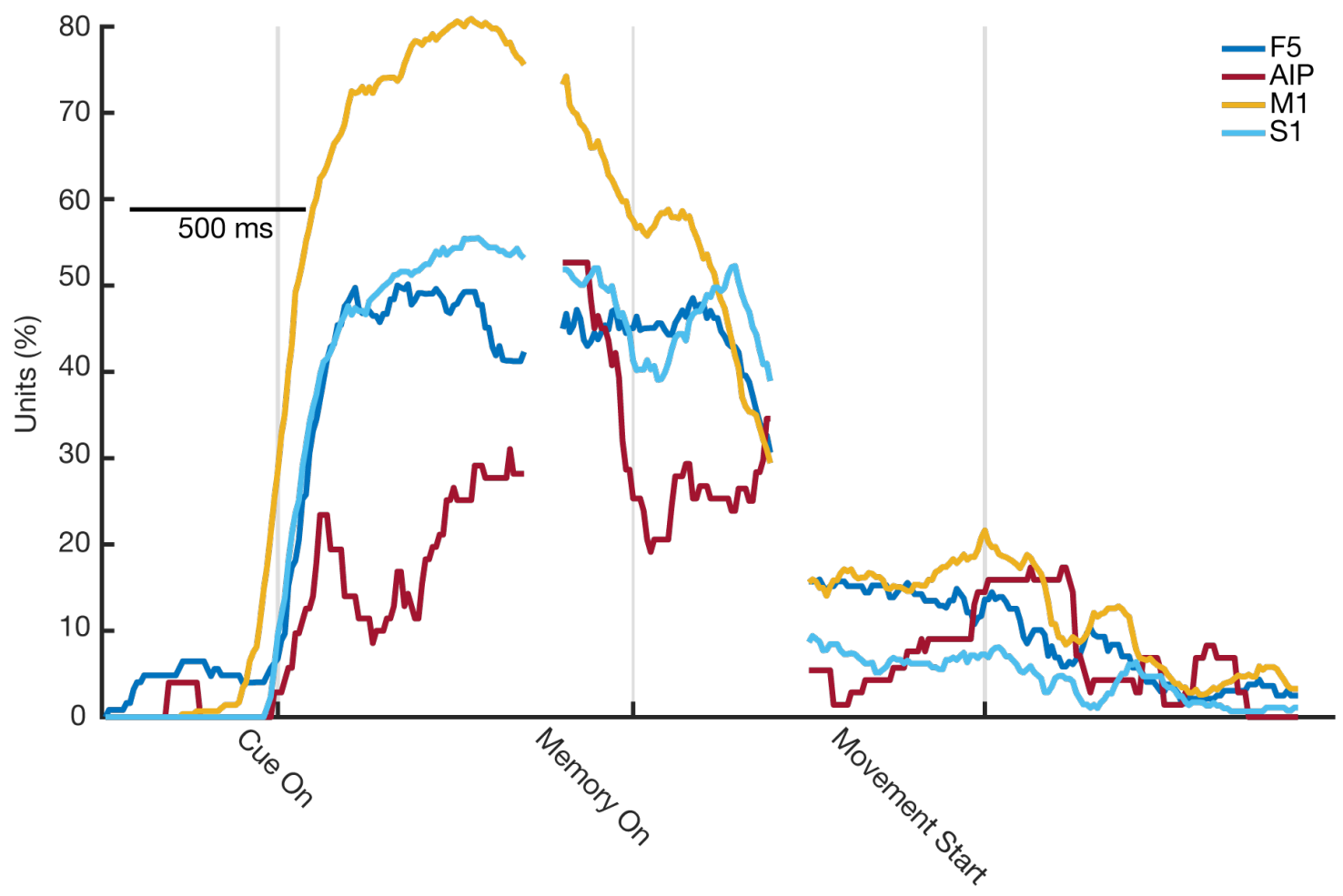

Figure 3.15: Percentage of significant units (factor sensory condition) during active grasping task. This plot shows the number of significantly tuned units (ANOVA, factor sensory condition, in percent) for each time point of the active grasping task for areas F5, M1, AIP and S1. All areas show tuning during the cue epoch. During the early memory epoch, all areas show significant tuning, however this decreases dramatically during late memory.

\subsubsection{ROC analysis revealed tuning towards sensory condition in all ar- eas}

After looking at the PSTHs of single units (section 3.3.2), the receiver operating charasteristic analysis was calculated for five different epochs in order to determine how well the ROC analysis can discriminate between visual and tactile trials, as described in sec- 
tion 2.7.5. For each area, two control periods were analysed. The first control was the baseline activity, where discriminability should be close to chance level (0.5) since no cues are given to the animal, yet. The second control was the cue period. Due to the task design, tactile and visual cue epochs are very different, so here a higher ROC score would be expected. For the actual analysis three more epochs were analysed: Early memory period, late memory period and grasping period.

Results are plotted as a histogram per area and epoch, to get an idea how strongly units of a certain region are tuned towards the sensory conditions of the task. The closer the ROC score is to one, the better both sensory conditions can be differentiated.

\subsubsection{ROC analysis for premotor cortex revealed better discriminability during early memory}

Figure 3.16 shows the ROC score over time for one example unit of premotor cortex area F5. During cue epoch, the ROC score went up to around 0.8 and remained at that level during early memory epoch. In late memory, the score falls down to around 0.6, showing worse discriminability. During grasping, the ROC score is close to chance level (0.5).

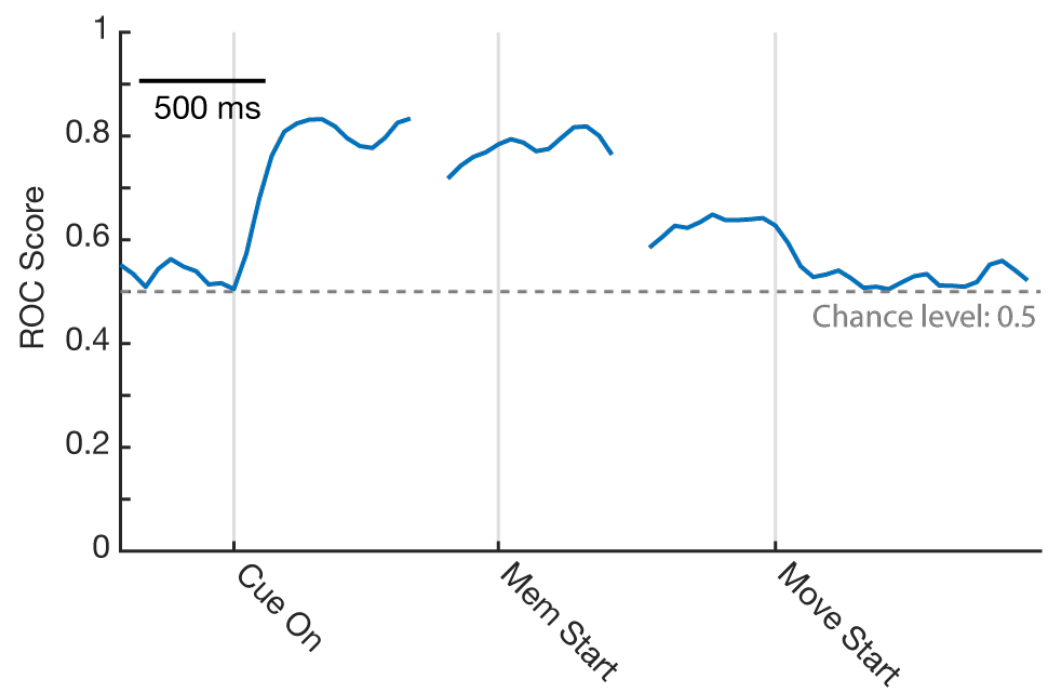

Figure 3.16: ROC score over time for a F5 unit. This plot shows the ROC score for an example $\mathrm{F} 5$ units over time, starting from baseline, over cue presentation, memory period and finally grasping epoch. The ROC score is highest during cue period and only slightly above baseline for the rest of the task.

For area F5 the results are plotted in figure 3.17. Here, all units from all sessions were pooled ( $n=138$ ). As expected, most units showed an ROC score close to chance level (0.5) during baseline period. During cue, discriminability was quite well with 28 units achieving an ROC score above 0.9 and therefore showing close to perfect discriminiability. The mean ROC score during cue epoch was 0.7. Still, 60 units showed a score around 
0.5. During early memory, 55 units reached an ROC score around 0.5 , corresponding to chance level. Only nine units showed an ROC score between 0.9 and 1, hinting towards almost perfect discrimination of visual and tactile trials. For this period the mean ROC was 0.67 and therefore lower than during cue. During late memory, discrimination got worse (mean 0.60), with 89 units falling close to chance level. No unit had a score above 0.9. During grasp, ROC scores decreased further (mean 0.56), where most units (112) were close to chance level, with no unit having a higher score than 0.8. Overall, scores were lower during grasping, compared to late memory.
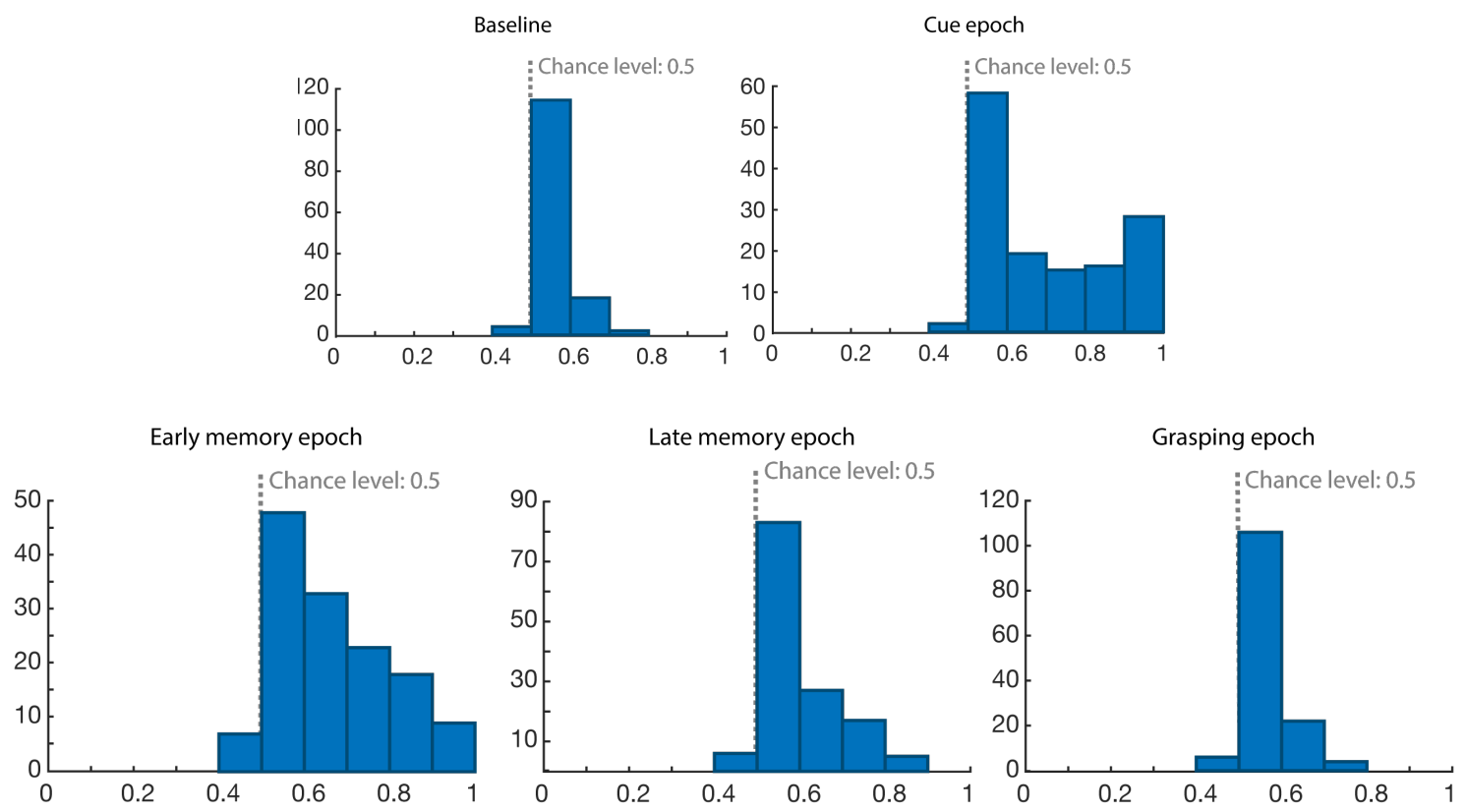

Figure 3.17: Distribution of ROC scores for area F5. The histogram shows how often each score was found in F5, in bins of 0.1. The best discriminabilty could be found during early memory, where more units got a high ROC score. From there the ability to discriminate went down during late memory and grasping.

As time goes on, it got harder to discriminate between visual and tactile trials in the population of F5 units. During the cue period and early memory, the sensory condition could be discriminated quite well but it got harder to differentiate during late memory and was almost at chance level during grasping.

\subsubsection{ROC scores of primary motor cortex were higher during early memory}

Figure 3.18 shows one example unit from primary motor cortex. During the cue period, the score rises to over 0.9 , showing almost perfect discriminability during the cue epoch. The score falls down during early memory and is close to chance level during late memory and grasping epoch. 


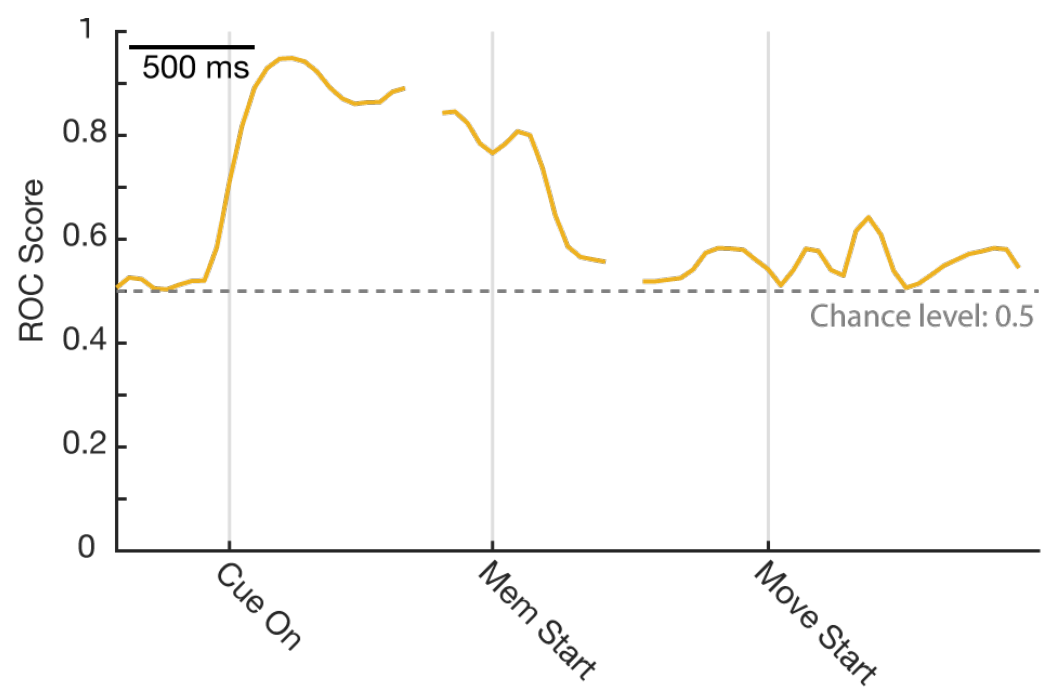

Figure 3.18: ROC score over time for an example unit of area M1. This plot shows the ROC over time, starting from baseline, over cue presentation, memory period and finally grasping epoch. Discriminability is best during cue and only slightly above baseline for the rest of the task.

A similar pattern to that of the F5 units (figure 3.17) could be found in M1, where overall discrimination got worse from early memory over late memory to grasping. Results for M1 can be found in figure 3.19.
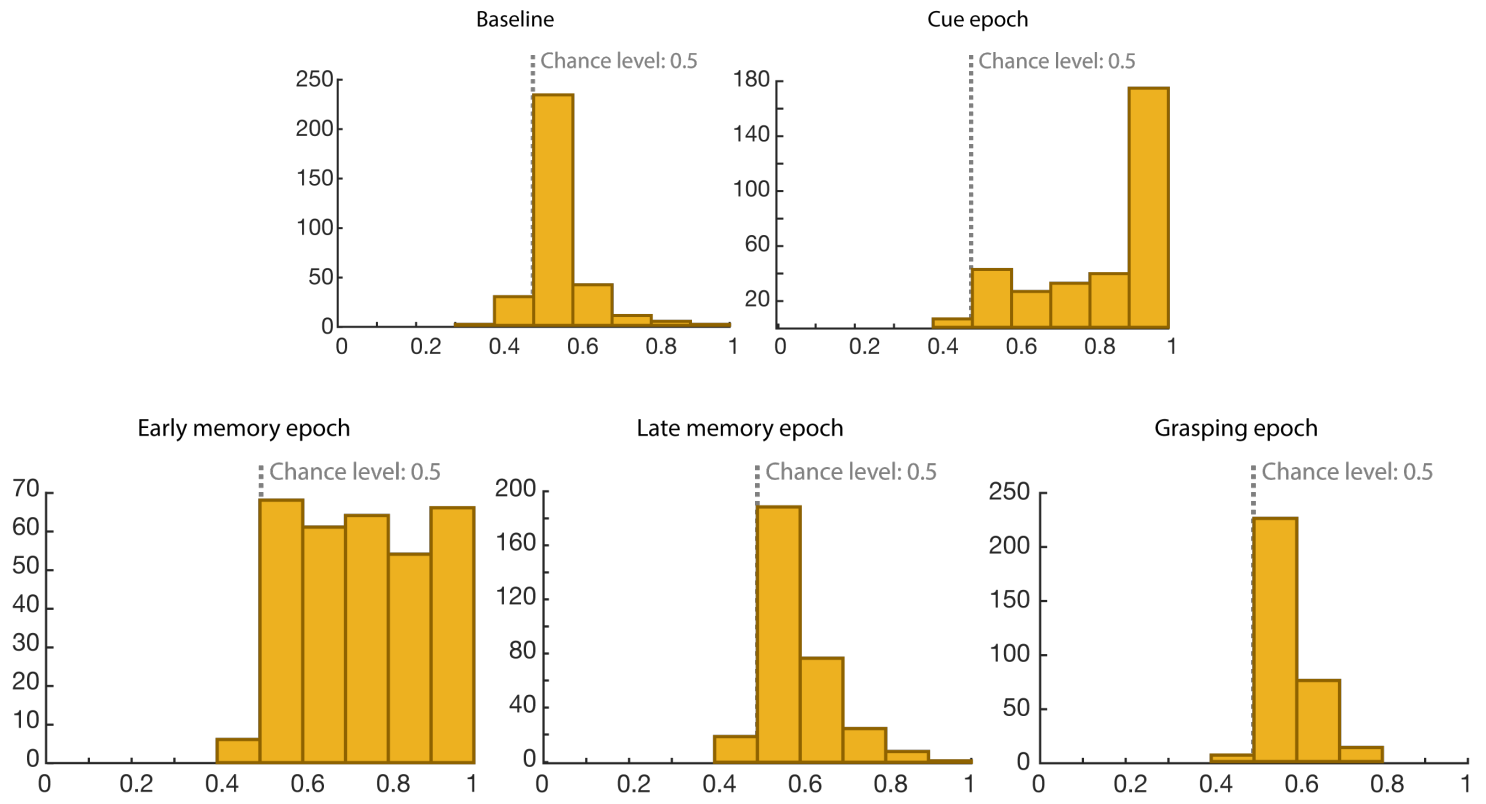

Figure 3.19: Distribution of ROC scores for area M1. The histogram shows how often each score was found in M1, in bins of 0.1. The best discriminabilty can be found during early memory, where more units get a high ROC score. From there it goes down during late memory and grasping.

Again, baseline period showed the expected pattern where most scores were close to chance level (mean 0.56) and cue period allowed to differentiate between both condi- 
tions in both units (mean 0.84). 174 units reached an ROC score above 0.9. During early memory, 74 units were close to chance level. 66 units were able to almost perfectly discriminate between visual and tactile trials and achieved an ROC score between 0.9 and 1 . The mean ROC during the early memory period was 0.74 . This changed during late memory, when only 9 units reached a score above 0.8 and the mean dropped to 0.59 , showing that discrimination between conditions got harder. The distribution of ROC scores in M1 looked very similar between late memory and grasp, although discriminability decreased further between both epochs.

In summary, the results for M1 look similar to those of area F5 both with an overall higher mean ROC score. Aside from the two controls (baseline activity and cue period) the ROC scores are highest during early memory and decrease as time goes on. During the grasping epoch

\subsubsection{ROC scores of primary somatosensory decreases over time}

In figure 3.20, the ROC score over time is given for one example unit from the primary somatosensory cortex. For the majority of the task, the discriminability was low, with an ROC score around 0.5 , which equals chance level. During cue presentation, when the monkey was either sitting still and seeing the object or moving to tactually explore it, the ROC score rose to above 0.9.

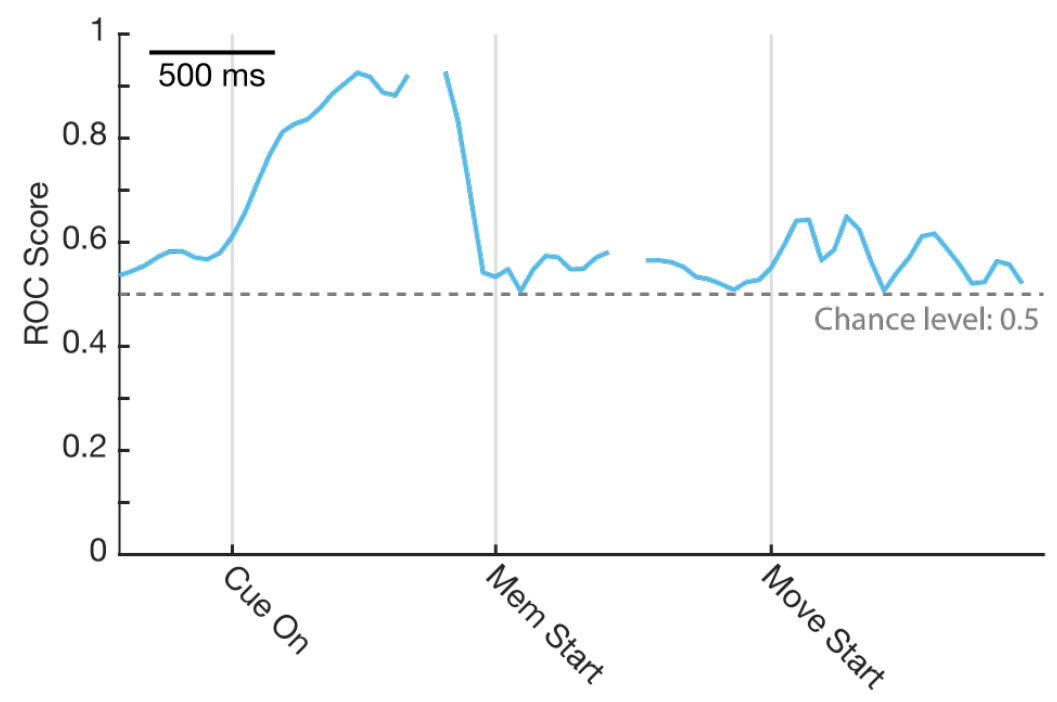

Figure 3.20: ROC score over time for an example unit of area S1. This plot shows the ROC over time, starting from baseline, during cue presentation, memory period and finally grasping epoch. Discriminability is best during cue and only slightly above baseline for the rest of the task.

The overall ROC score distribution across all recorded $\mathrm{S} 1$ neurons again showed a similar pattern, where discriminability for most neurons was best during the cue epoch and then 
declined afterwards. During cue the mean ROC was 0.73. During early memory, 67 units were at chance level, and 48 units achieved a score above 0.9 , showing almost perfect discriminability was possible from these neurons (mean 0.73). During late memory and grasping however, no unit's score was above 0.9 and 0.8 , respectively. Mean scores were 0.58 and 0.55. Again, most units achieved an ROC score around chance level (0.5 to 0.6).
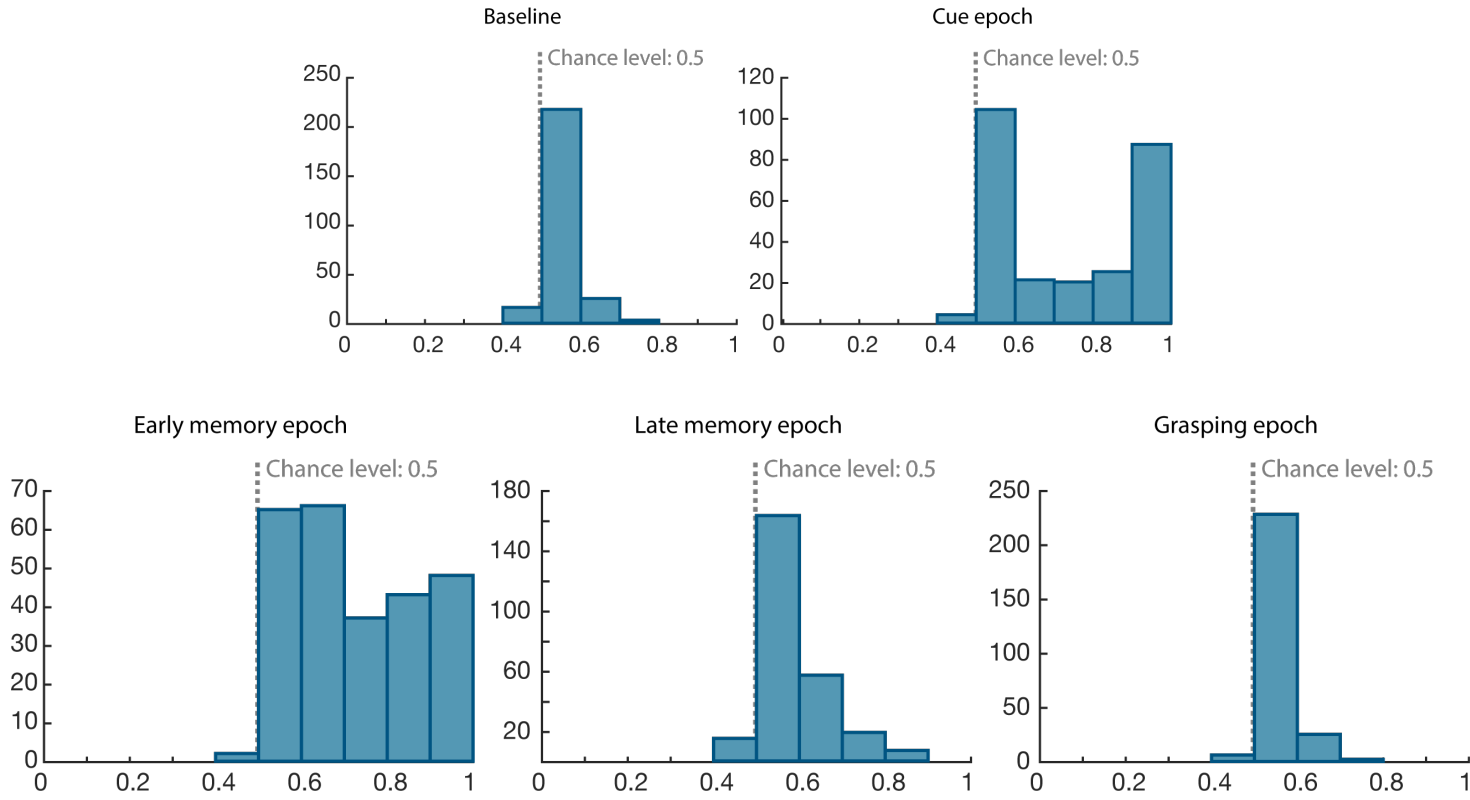

Figure 3.21: Distribution of ROC scores for area S1 during early memory, late memory and grasping. The histogram shows how often each score was found in F5, in bins of 0.1. From left to right plots show the distribution during rarly memory, late memory and grasping epoch. As in the other areas, the highest ROC score can be found during the early memory epoch, later no units reach a score above 0.8 .

In area S1, the ROC analysis could differentiate best between visual and tactile trials during the cue and early memory epochs. Similar to the patterns seen in M1 and F5, discriminability goes down over time.

\subsubsection{ROC scores of anterior intraparietal cortex were best during early memory}

An example of the ROC score of an AIP unit over time is plotted in figure 3.22. Discriminability was best during cue presentation and decreased during memory period. During late memory, it was only slightly above baseline and remained there during grasp.

Since only a low number of units could be recorded from AIP $(n=46)$, a population analysis of the ROc scores was difficult. The results of this analysis are presented here but they are most likely not as representative of the activity of this brain area as is the case for the other areas, where more units could be recorded. 


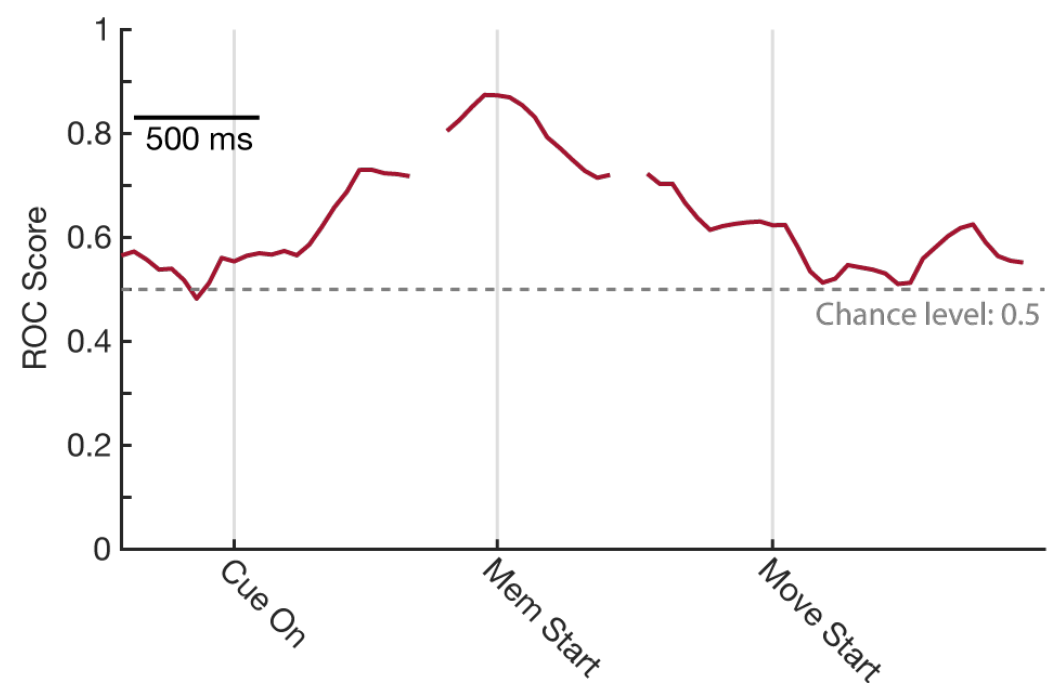

Figure 3.22: ROC score over time for an example unit of area AIP. This plot shows the ROC over time, starting from baseline, during cue presentation, memory period and finally grasping epoch. Discriminability is best during cue, goes down during memory period and only slightly above baseline for the rest of the task. The highest ROC score can be found during early memory, later during the task (during late memory and grasping epochs), no unit has an ROC score above 0.7.
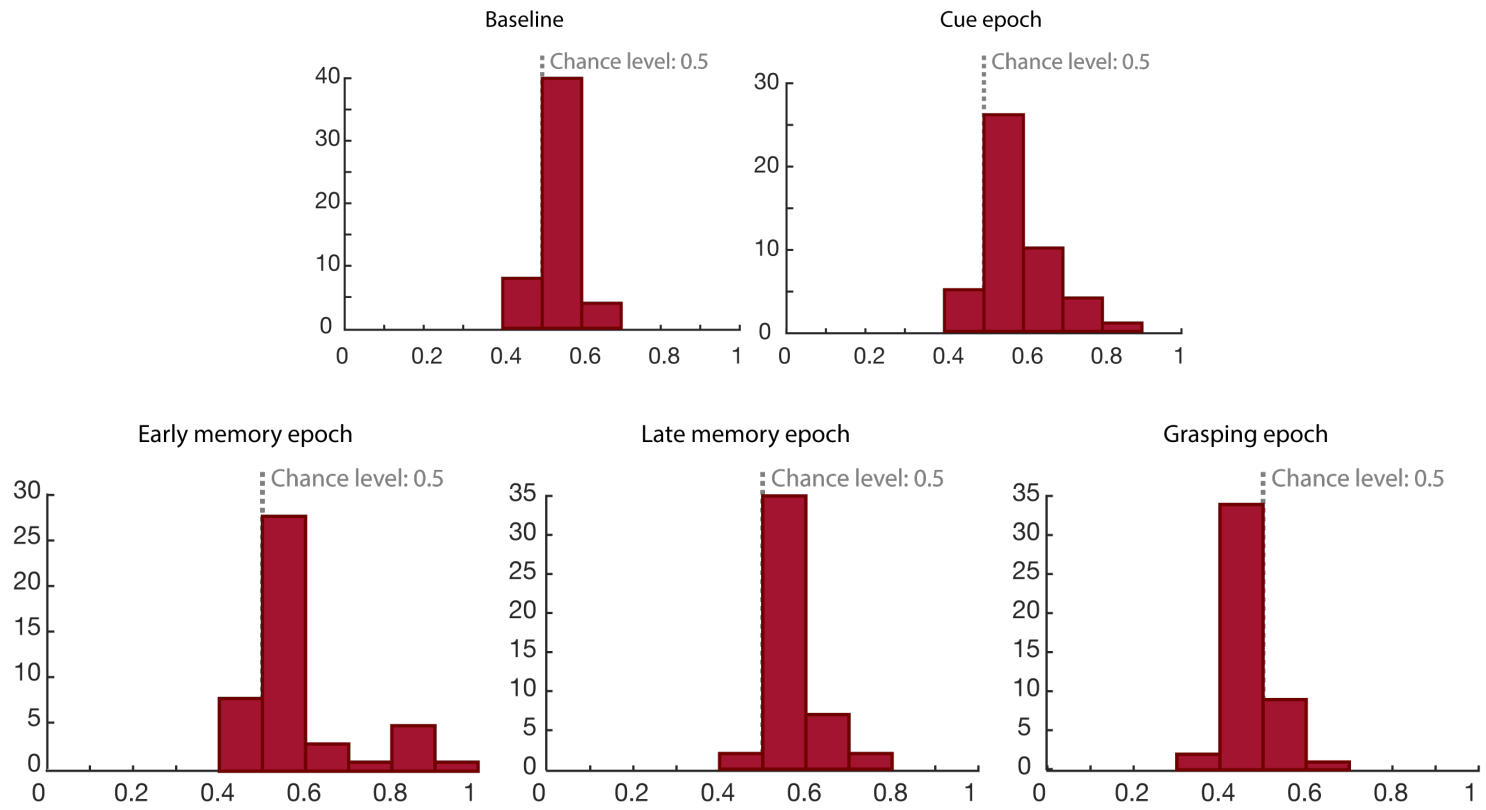

Figure 3.23: Distribution of ROC scores for area AIP during early memory, late memory and grasping. The histogram shows how often each score was found in AIP, in bins of 0.1 . From left to right plots show the distribution during early memory, late memory and grasping epoch.

During cue, discriminability was close to chance level (mean 0.57), 44 units fell close to 0.5. During cue epoch, the ROC score of 31 units was close to chance, but even though the conditions differ greatly (visual cue presentation versus tactile exploration) no unit 
reached a score above 0.9 and only 1 showed a score above 0.8 , showing that differentiating between both conditions is not easily possible. During the early memory epoch a few units reached an ROC score above 0.8 (five) and 0.9 (one), but discriminability for most units still remained at chance level (36). The mean ROC score during the early memory epoch was 0.58. During the late memory epoch, it again got harder to differentiate the two conditions (mean 0.57). The distribution of ROC scores during grasping looked similar, with a mean of 0.56 .

Overall the activity of the AIP units seems to be too low and noisy to successfully dicriminate between visual and tactile trials. All mean ROC scores fall between 0.5 and 0.6 and remain therefore close to chance level. In all epochs most units remain close to chance level. While a similar pattern to the other area started to emerge, where discriminability is best during early memory and declines from there, the overall ability to discriminate between both conditions remains low.

\subsubsection{Decoding}

One goal of this project was to see how the brain uses sensory and object information in order to plan grasps. For this purpose, I investigated how well objects and the sensory modality (visual or tactile) can be decoded from the neuronal activity of one area during individual trials. An LDA decoder was trained on the data of each area during individual trials, as described in section 2.7.6, and the resulting decoding accuracy was evaluated.

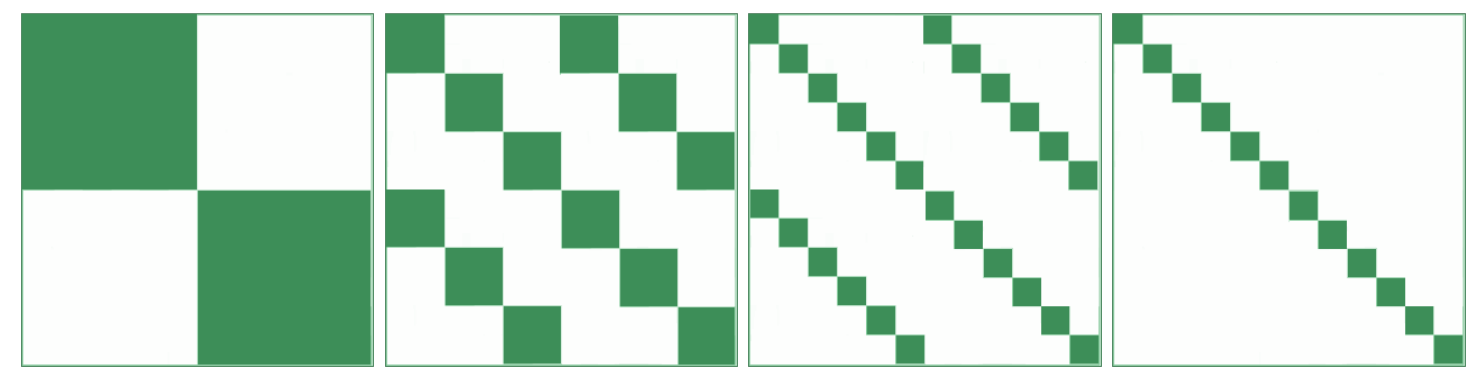

Figure 3.24: Decoding models. A schematic overview over different possible outcomes of the decoding, illustrating how certain distributions would look. A: Sensory mode perfectly decoded but objects unknown. B: Object pair identity decoded but not sensory condition. C: Object identity decoded, but not sensory condition. D: Objects and sensory condition decoded perfectly.

To describe the decoding results, confusion matrices were generated for F5, M1 and S1 during early memory, late memory and grasping epochs. Figure 3.24 shows four specific outcomes for particular coding assumptions, meaning which pattern would be visible if a certain modality could be decoded. The first model (sensory mode, A) describes the pattern of a confusion matrix, if visual and tactile trials could be decoded from each other, 
but no object or grasping specificity could be found. The Object pair specific model (B) describes the case that the two objects that are grasped the same (cube and sphere, both rings, bar and box) could not be differentiated and the sensory mode also could not be decoded. In the case of the object specific decoding model (C), the decoder would be able to differentiate between all six objects, but not between the sensory condition. The last pattern (D) would occur if all conditions and objects could be decoded.

Figure 3.25 shows confusion matrices for F5, M1 and S1. Three epochs are decoded: Early memory (500ms after memory period starts), late memory (500ms before movement starts) and the movement epoch (500ms after movement started). Area AIP was not tested, due to the low number of recorded neurons from this area, which would make results unreliable.

The first row shows the results for F5. During all three epochs, the decoder could decode quite well between all six objects and sensory conditions (compare figure 3.24). Mean accuracy rose from 51 over 56 to $57 \%$, chance level for 12 conditions (six objects, presented visually or tactually) would be $8,3 \%$, so decoding accuracy during all periods is well above chance. For M1, differences between the three epochs became apparent. During early memory, the decoder could perfectly differentiate between visual and tactile trials, with a slight confusion between objects. The overall pattern was close to the sensory mode but the diagonal for perfect decoding was visible, even though not perfectly (accuracy 37\%). During late memory, the perfect sensory decoding accuracy vanished, but the diagonal for differentiation between objects and sensory conditions was still visible. Accuracy was at $35 \%$ during late memory. During grasping, accuracy increases to $62 \%$, when the decoder could differentiate not only between different objects but also between sensory conditions. S1 showed a similar pattern. During early memory, decoding accuracy lied at $31 \%$, with confusion happening between the different objects but not between sensory conditions. During late memory, the decoder could differentiate less well between all conditions. Accuracy was at $18 \%$ and confusion happened between all conditions. During grasping, accuracy increased to $37 \%$, with a stronger diagonal indicating that overall, all conditions could be differentiated above chance level. 
F5

Early memory Late memory Grasping
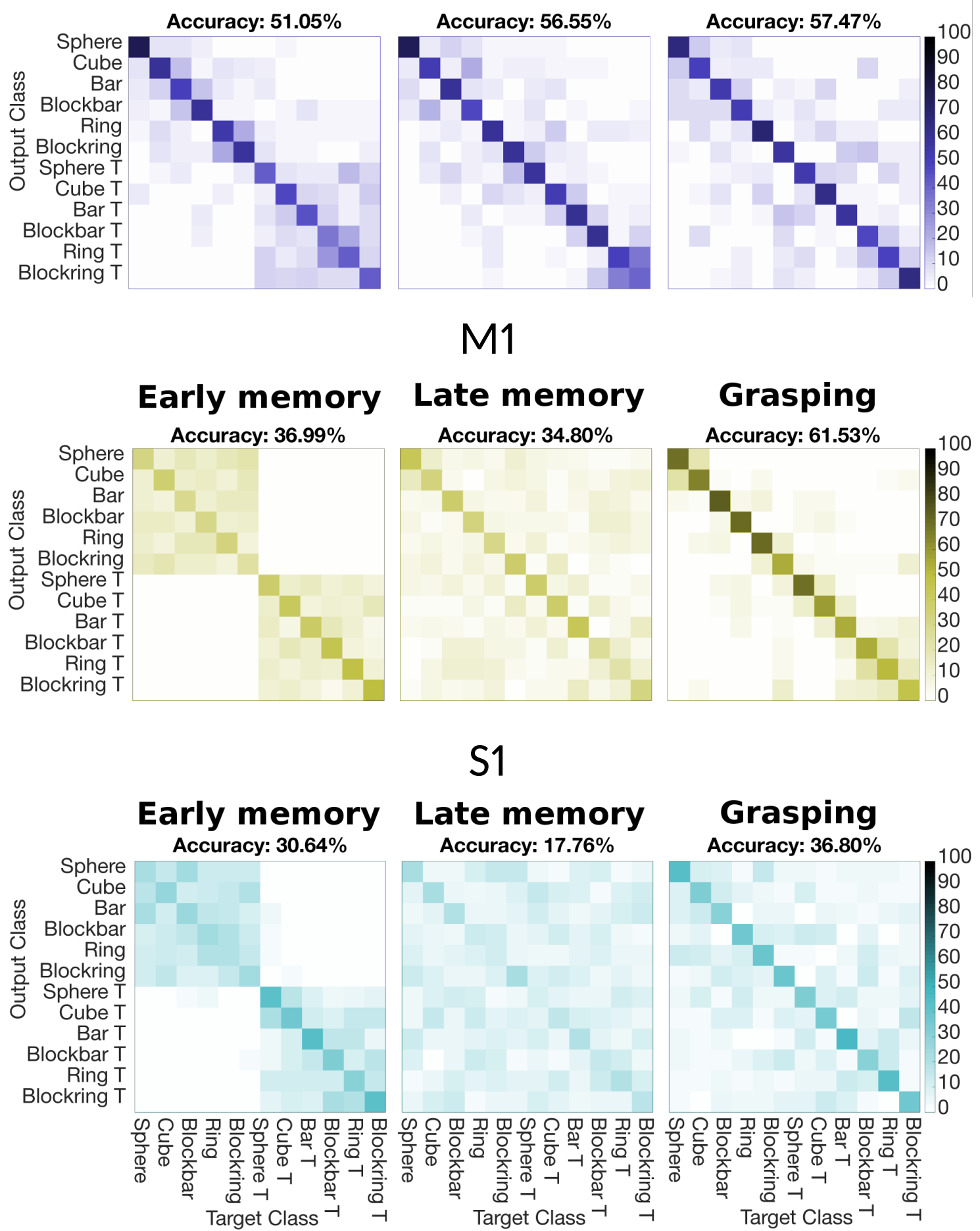

Figure 3.25: LDA decoding accuracy per epoch, averaged over five recordings. Confusion matrices showing decoding accuracy of 500ms per epoch for F5, M1 and S1. Decoded epochs are early memory, late memory and grasping.

Besides looking at the three epochs individually, decoding was also performed in a sliding window in order to see how the accuracy changes over time. The first question was, how 
well different objects could be decoded from the neuronal activity, independent of sensory conditions (see figure 3.26). In order to see how the accuracy chances over time, bins of 100ms were used for decoding based on the formerly described PSTHs (section 3.3.2), taken from early memory, late memory and grasping epochs. For cue period decoding was not attempted, due to the very different nature of the visual and tactile cue period. During early memory, decoding accuracy for the areas F5 and M1 was above chance level (chance level: $16,7 \%$ ) and increased during late memory. During grasp, decoding accuracy in M1 stagnated around 85\%. For F5, accuracy decreased from around $70 \%$ to $60 \%$. Accuracy for $\mathrm{S} 1$ on the other hand started around chance level and increased during late memory to about $75 \%$.

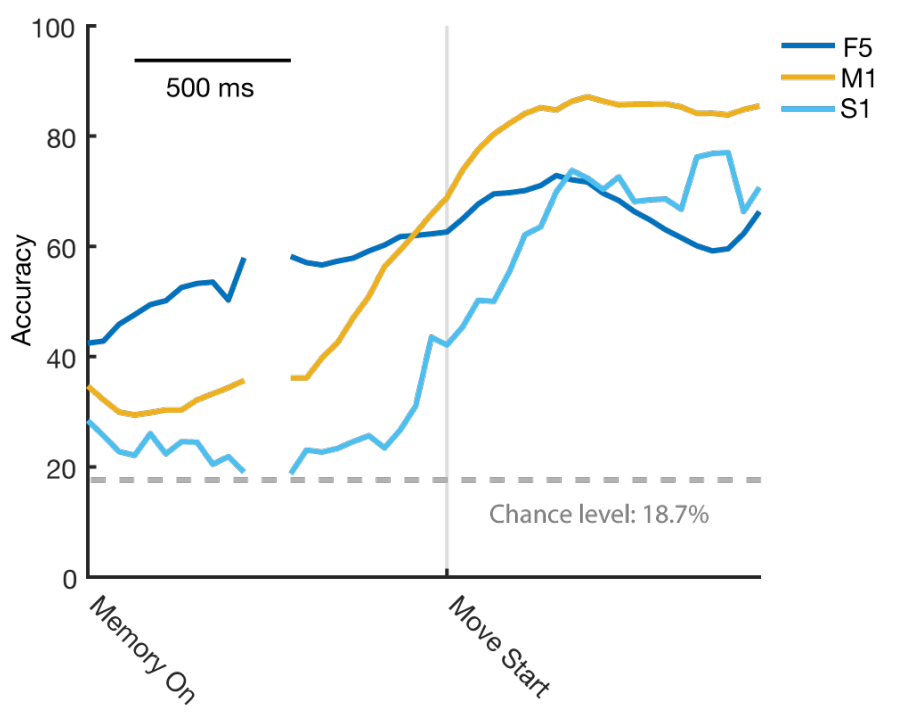

Figure 3.26: Decoding accuracy over time for an LDA decoder trained on different objects. This decoder was trained on object information and had no information about sensory conditions. For F5 and M1, decoding accuracy is above chance level (16,7\%) and increases further during late memory. For F5, accuracy goes down during grasping, while it stays steady in M1. Accuracy on S1 activity starts slightly around chance level and increases only during late memory, before it stagnates at around $70 \%$ during grasping.

When the decoder was trained only to distingish the two sensory conditions (visual cue presentation versus tactile exploration), best decoding in all three areas was achieved during the memory period (figure 3.27 ). Here, chance level is at $50 \%$, since only two conditions were decoded (visual and tactile trials). For all three areas, accuracy decreased during late memory and stayed stable during grasping, where it reached almost chance level when trained and tested on F5 and S1 data. For M1 data, accuracy stayed slightly above chance level, around 65\%. This shows that the closer the actual movement comes, sensory condition starts to almost gets lost and is harder to decode from the data. 


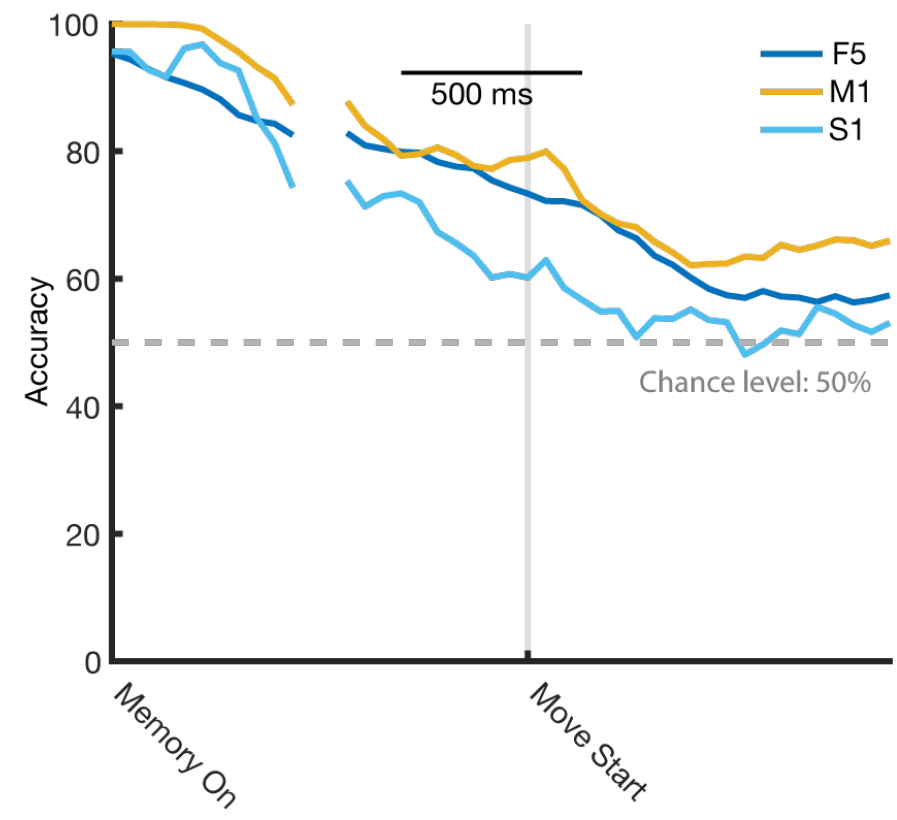

Figure 3.27: Decoding accuracy over time for an LDA decoder trained on sensory conditions. This decoder was trained on sensory information and had no information about object identity. For all areas, decoding accuracy starts above chance level, between 90 and $100 \%$ and decreases during memory period. In F5 and S1, decoding accuracy reaches almost chance level during grasping, while accuracy stays higher in M1.

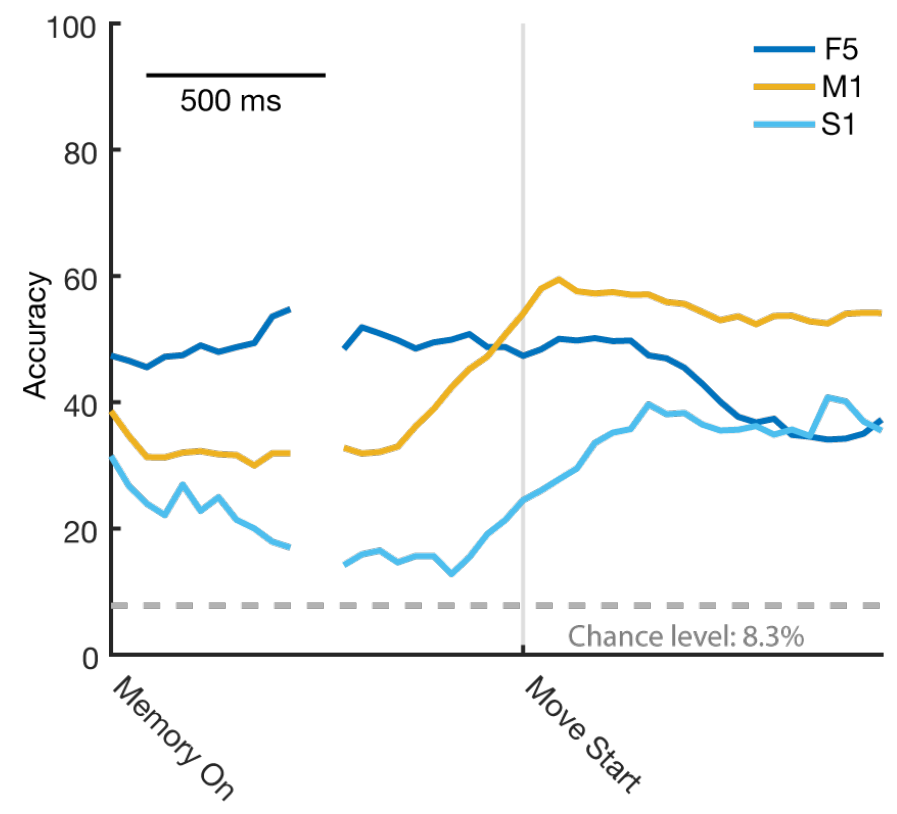

Figure 3.28: Decoding accuracy over time for an LDA decoder trained on object identity and sensory conditions. This decoder was trained on object and sensory information, showing decoding accuracy above chance level in all four areas.

In a last analysis, the decoder was trained based on sensory conditions and object identities, to see how well both conditions can be decoded simultaneously. Chance level is $8,3 \%$ (six objects and two sensory modalities). During early memory, accuracy in F5 and M1 is 
quite stable (50\% and $30 \%$ respectively). In S1, accuracy decreases during early memory. In late memory, accuracy for F5 stays stable and decreases only right at the end, slightly before movement starts. In M1, accuracy actually increases during late memory, until it reaches an value of $60 \%$, where it remains during grasping. $\$ 1$ accuracy behaves similar, where an increase during late memory and a stable decoding accuracy during grasping can be seen.

So in conclusion, most conditions can be decoded above chance level during early and late memory and grasping. For object information, decoding accuracy goes up during memory epoch, while the accuracy for the sensory modalities goes down with time. When trying to decode both, objects and sensory conditions at the same time, accuracy is still above chance level and increases over time. Best decoding accuracy for all conditions is usually found during grasping.

\subsection{Passive finger stimulation experiment}

In a second experiment, I investigated how different textures of objects are represented in the implanted brain areas (see section 2.2). For the passive stmulation experiment, different plates with gratings of various spatial frequencies were passively touching the finger of a monkey, while the animal remained still (see section 2.6).

\subsubsection{Single neuron activity}

To get a first glimpse of how the brain encoded these different structures, I again took a look at single neuron activity from all four implanted cortical areas. The raw data was preprocessed and filtered, but not spike sorted, so that all signals recorded from one electrode were treated as two multiunits, one filtered with a positive and one filtered with a negative threshold. Usually, spiked sorted data improved the quality of all results since signals from one electrode that actually stem from multiple neurons can be differentiated to a certain degree, resulting in a better representation of firing behaviour of units in the investigated area. To get a first overview over the data, all plots in the following sections were produced using multiunits, since this saves time and gives a first overview what can be expected. As presented in section 3.4.2, all tuning that were found during this task disappeared in later sessions. Due to this lack of consistent results, no spike sorting was performed, since this would not have improved the lack of results in later sessions. 


\subsubsection{No strong correlation with elements of the task in area F5}

For the ventral premotor area F5, very few units showed any patterns that corresponded to task elements of the passive stimulation task. A few examples were found in which some general activity changes towards one part of the task were visible, such as an increase in activity on touch in figure 3.29 or a general small increase during platform movement in figure 3.30. However even for those units no lasting significant difference between plates or movement directions could be seen.

The first units showed an increase in activity, when the plate first touched the finger, but not during any other events. For about $150 \mathrm{~ms}$ a significant difference between the different plate structures could be seen. Interestingly, once the plate starts moving and ongoing tactile stimulation starts when the plate moves below the finger, the firing rate remains stable.

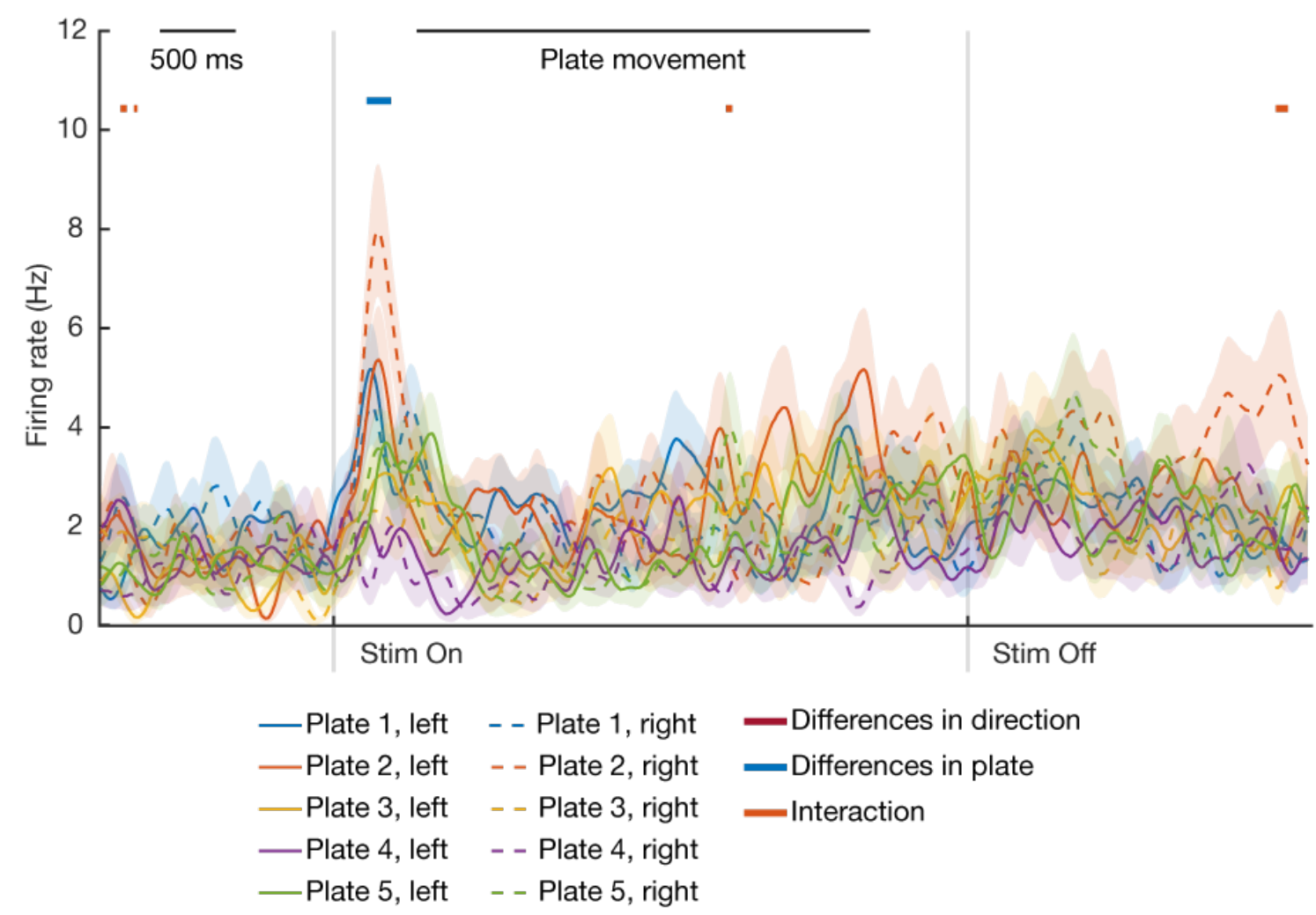

Figure 3.29: Firing rate of an F5 unit, reacting to the start of tactile stimulation. A unit from premotor cortex area F5, plotted is the firing rate in $\mathrm{Hz}$ over time. Vertical lines mark the moment the plate was moved upwards and started touching the finger or downwards, when the plate stopped touching the finger. The activity peaked right after the plate first touched the finger, but was quite constant otherwise.

The second F5 unit on the other hand did not increase firing rate when the stimulus first touched the finger but while the platform was moving below the finger, although this effect is not very strong, baseline activity for this unit lied between $\mathrm{OHz}$ and $4 \mathrm{~Hz}$. Dur- 
ing plate movement the firing rate of this unit increases to up to $6 \mathrm{~Hz}$. No significant differences between structure or movement direction of the stimulus could be found.

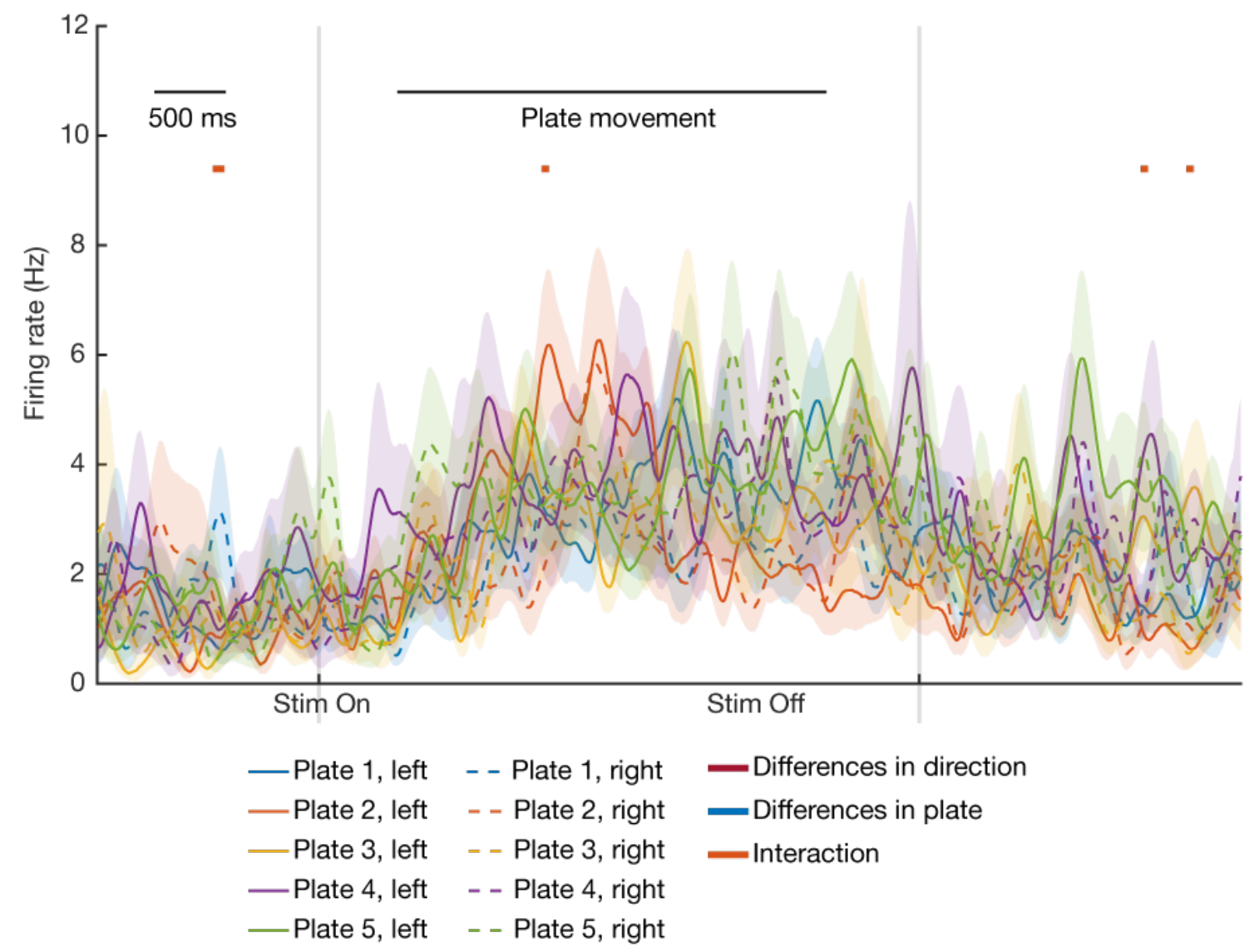

Figure 3.30: Firing rate of an F5 unit, showing a small activity increase during stimulation. A unit from $\mathrm{F} 5$, plotted is the firing rate over time in $\mathrm{Hz}$. Vertical lines mark the moment the plate was moved upwards and started touching the finger or downwards, when the plate no longer touched the finger. Activity increased generally during stimulation, but without any significant differences between plates or movement directions.

\subsubsection{Units in area AIP showed only minor changes during stimulation}

Neurons in the anterior intraparietal cortex are mainly known for reacting to visual stimuli. Due to the lack of visual stimuli in this task, I expected close to no tuning of units in this area during the passive stimulation experiment. Overall, not many units could be recorded during the passive stimulation task (see table 3.1) and most do not show any activity changes during stimulation (such as figure 3.31). Furthermore no significant difference between plate structures or movement directions could be found. This lack of any modulation is quite representative for area AIP during the passive stimulation. Very few units showed activity changes during the task, one is plotted in figure 3.32. This unit showed an activity increase for the moment the plate touched the finger in most conditions. Interestingly this activity change was significantly different between movement 
directions for about 200ms. After the plate touched the finger but before it started to move in any direction, the activity of this unit returned to baseline. Furthermore, especially for plate movements from left to right (solid lines) activity increased when the plate started moving.

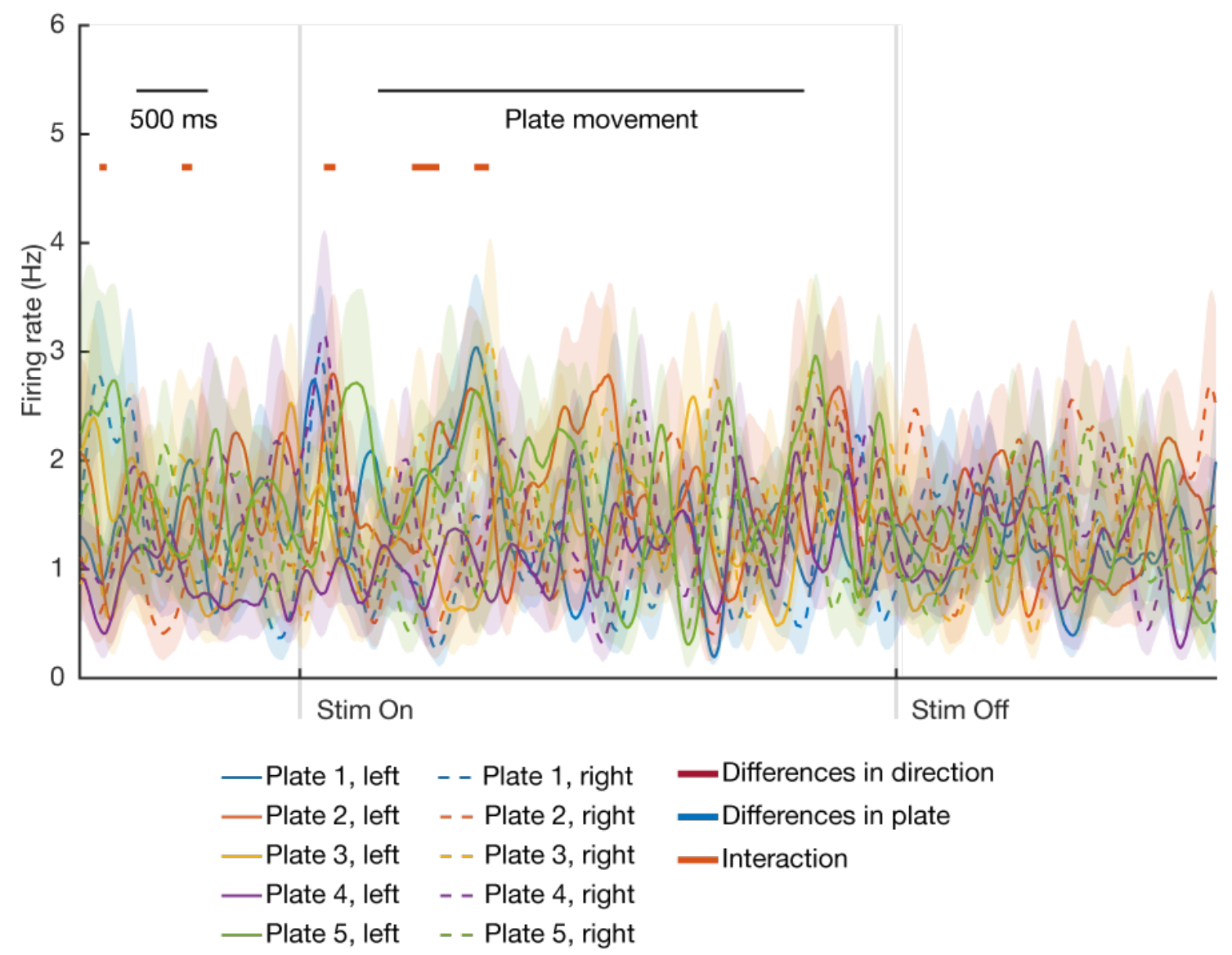

Figure 3.31: Firing rate of an AIP unit, showing no activity changes during the whole task. A unit from anterior intraparietal cortex, plotted is the firing rate over time in $\mathrm{Hz}$. Vertical lines mark the moment the plate was moved upwards and started touching the finger or downwards, when the plate no longer touched the finger. The activity was rather unmodulated by the tactile stimulation before, during and after the task, with no visible reactions to any part of the task. 


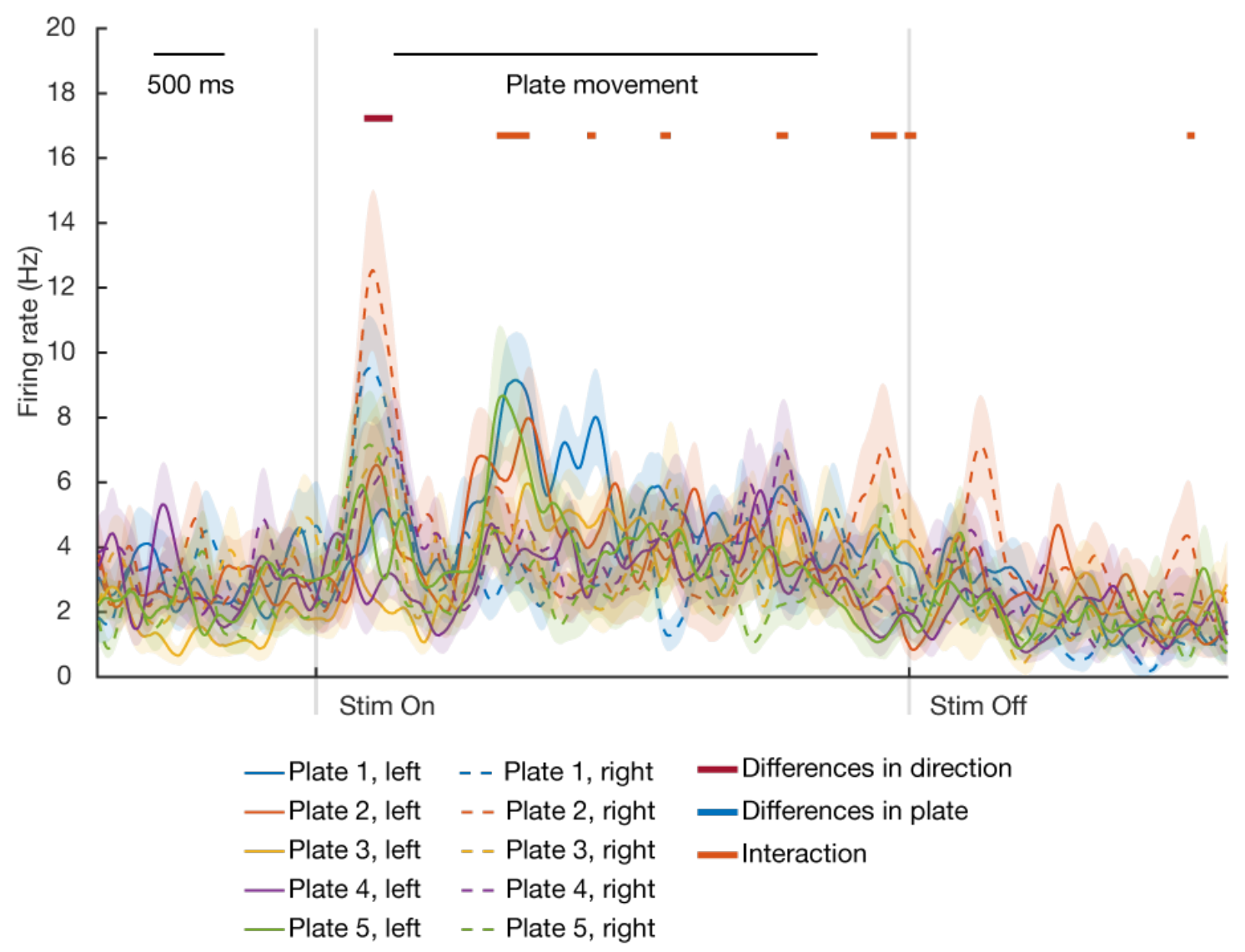

Figure 3.32: Firing rate of an AIP unit, reacting to tactile stimulation and plate movement start. A unit from anterior intraparietal cortex, plotted is the firing rate over time in $\mathrm{Hz}$. Vertical lines mark the moment the plate was moved upwards and started touching the finger or downwards, when the plate no longer touched the finger. The activity peaked when the plate first touched the finger or when the plate movement started, especially for movements from left to right.

\subsubsection{Units in M1 reacted to tactile stimulation}

The primary motor cortex $\mathrm{M} 1$ is known to generate movements and sits next to the primary somatosensory cortex. In this section, two examples of units recorded from M1 during the passive stimulation experiment are presented. Overall, more units showed modulation to task parameters, compared to AIP and F5, where responsive units were sparce.

Figure 3.33 shows one unit recorded from primary motor cortex during the passive finger stimulation task. Firing rate increased for all conditions, once the plate touched the finger (at the "Stim On" mark). Activity decreased as long as the plate stood still, increased again once movement started and finally decreased with ongoing movement. Once the plates moved down and lost contact of the finger, an off-response in form of a small rise in activity could also be seen. Small differences were visible for different plate structures and movement directions, such as a higher activity during leftward movements approxi- 
mately $700 \mathrm{~ms}$ after the stimulation started.

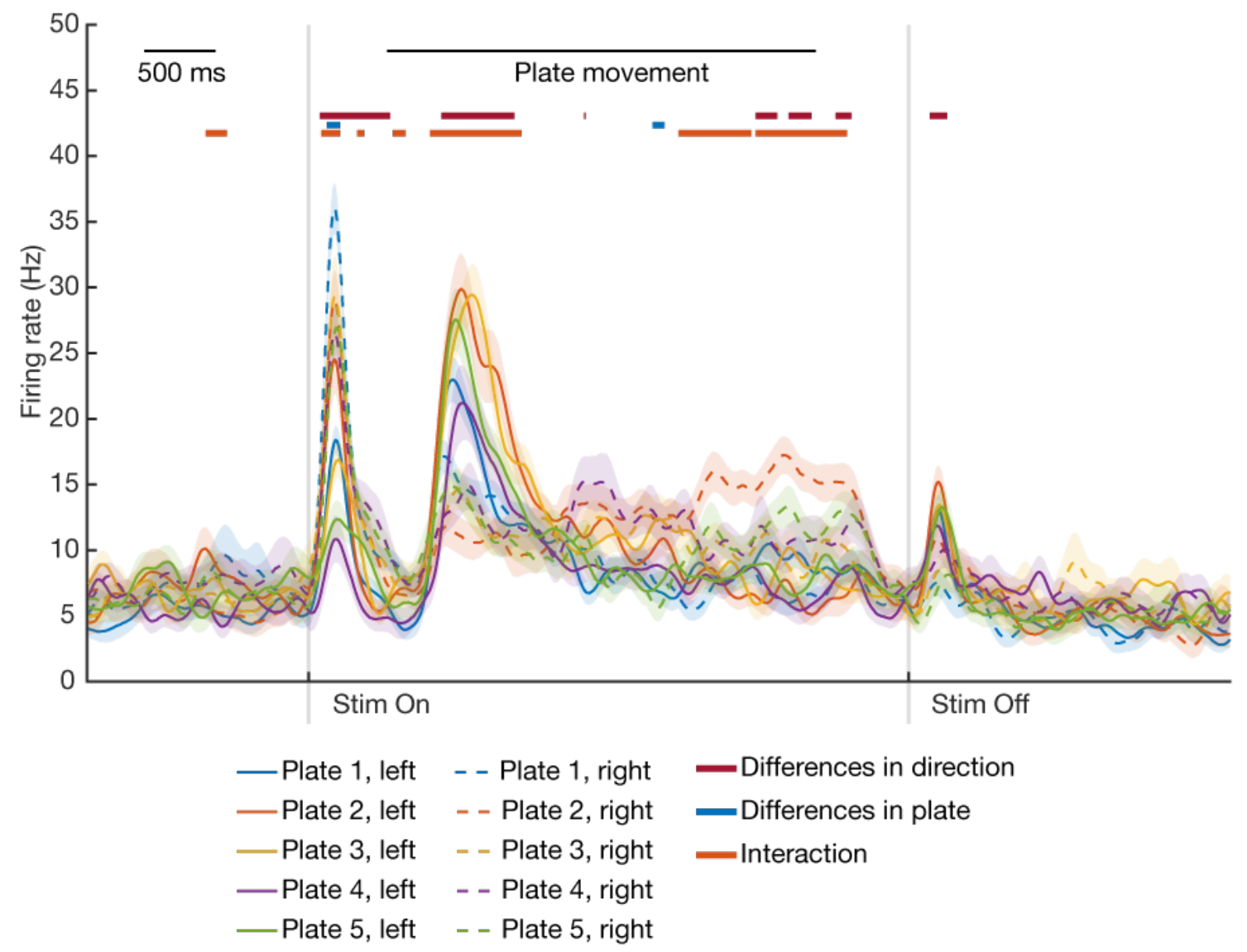

Figure 3.33: Firing rate of an $\mathrm{M} 1$ unit, reacting to tactile stimulation start and plate movement start. A unit from primary motor cortex, plotted is the firing rate over time in $\mathrm{Hz}$. Vertical lines mark the moment the plate was moved upwards and started touching the finger or downwards, when the plate no longer touched the finger. The activity peaked at three timepoints for all conditions, when the plate first touched the finger, when it started moving and when the plate left the finger again.

The second example unit from M1, (figure 3.34) shows a unit that strongly responded to movements of the plate below the finger of the monkey. After the plate first touched the finger, while it already remained still, a small decrease in activity was seen. Once the plate started moving, activity generally increased and differences between various plate structures became apparent. For all plates, activity is highest when the plate starts moving but remains increased during the whole movement phase. This increase in activity stopped when the plate stopped being moved and a small off response could also be seen when the plate lost contact to the finger. 


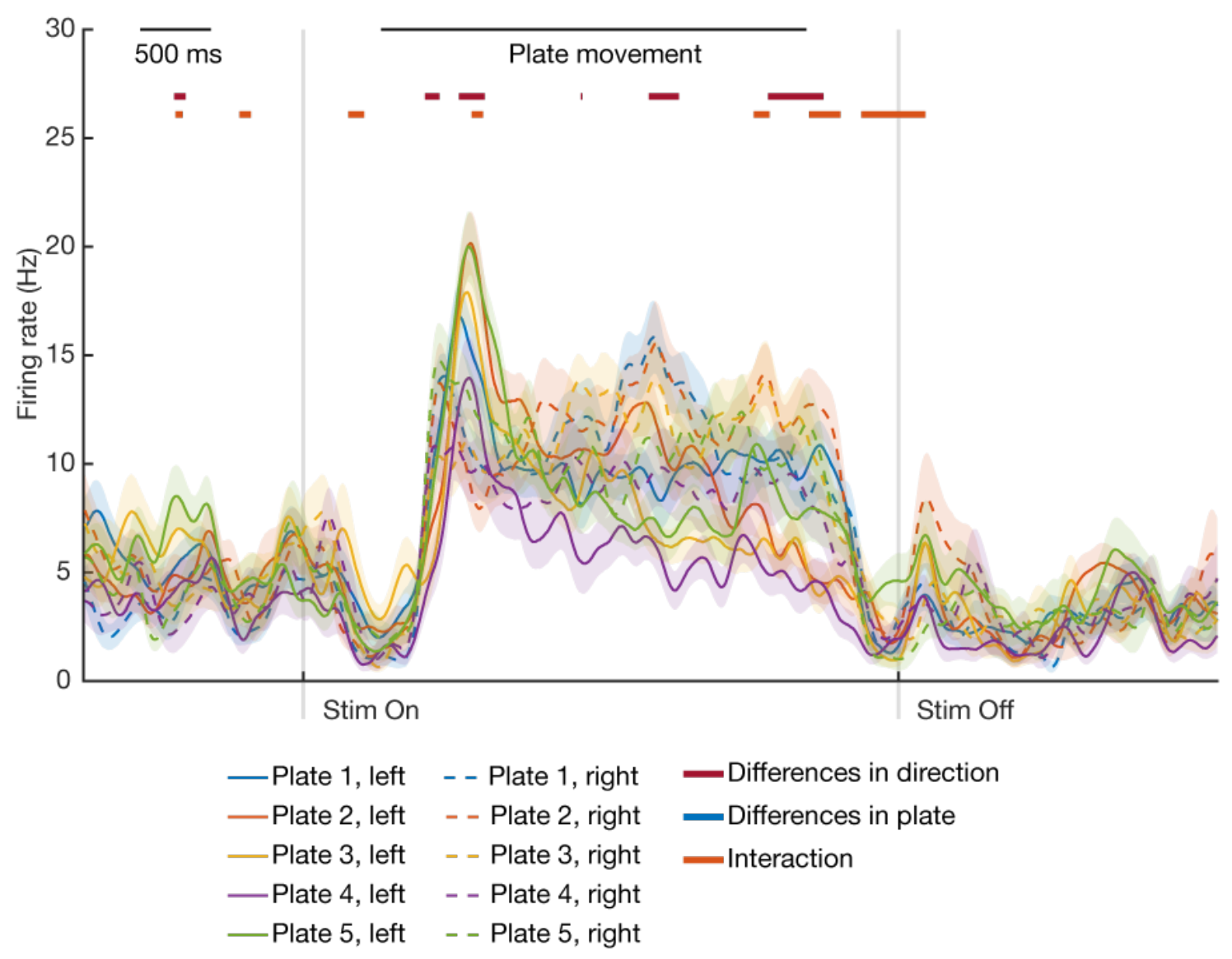

Figure 3.34: Firing rate of an M1 unit, reacting to movement of the plate below the finger. A unit from primary motor cortex, plotted is the firing rate over time in $\mathrm{Hz}$. Vertical lines mark the moment the plate was moved upwards and started touching the finger or downwards, when the plate no longer touched the finger. The activity peaked during platform movement when the plate was moving either from left to right or right to left below the finger of the monkey.

\subsubsection{Tactile stimulation drove the activity of S1 units}

The primary somatosensory cortex is an important area for the sense of touch, therefore this task should be perfect to drive the neurons in this area. Here, two example neurons from this area are shown, both showed some degree of activity changes during tactile stimulation. In figure 3.35 most conditions elicit a small response once the plates moved up and touched the finger, which decreased quickly while the plate remained still. The start of the movement caused another increase in activity for all conditions, which decreased while the movement continued, with rightward movements causing a small raise after the movement ended. Some conditions caused another raise after the plate left the finger. During plate movement, some significant differences were seen between conditions, although they did not persist during the whole movement. 


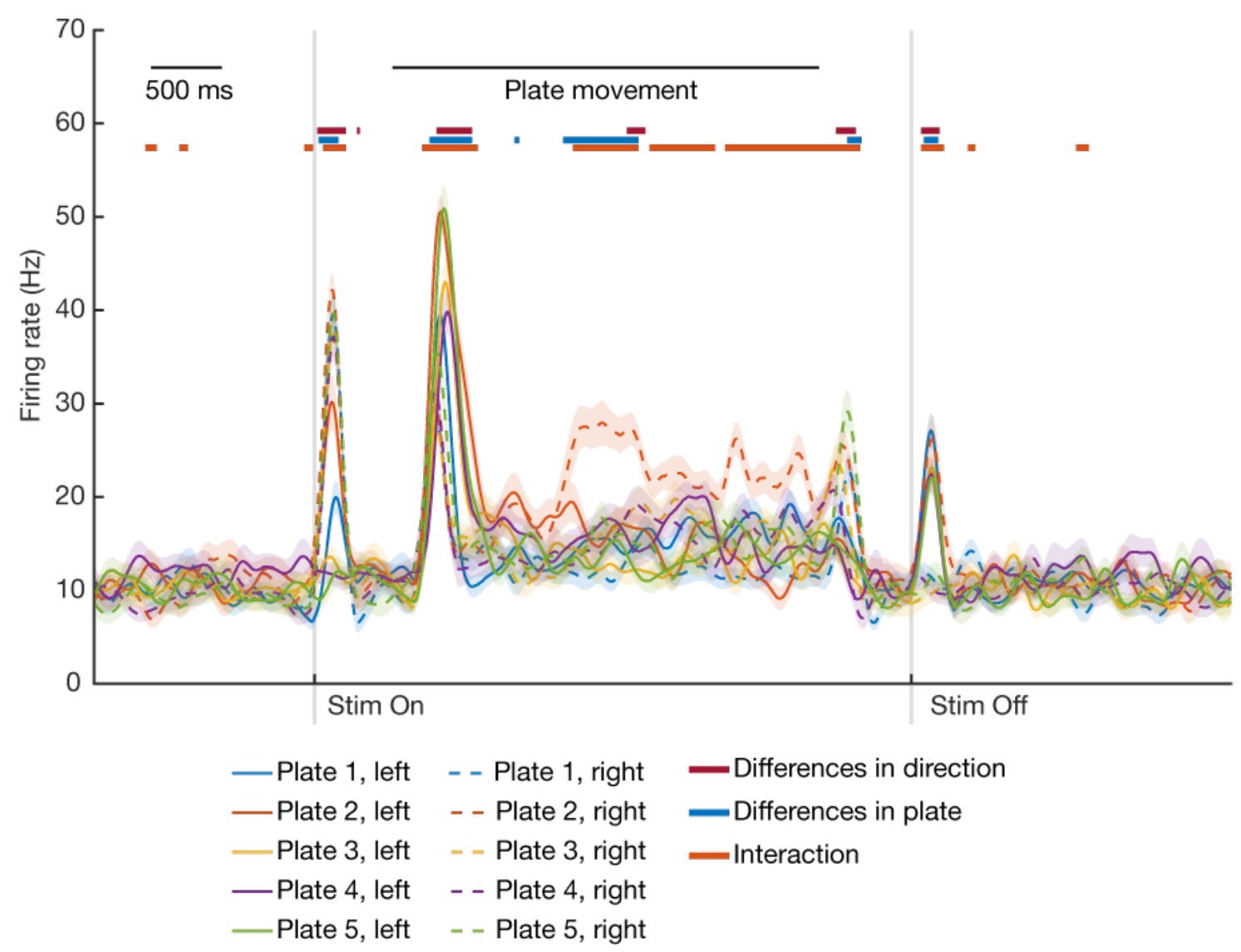

Figure 3.35: Firing rate of an $\mathbf{S 1}$ unit, reacting to tactile stimulation start and plate movement start. A unit from primary somatosensory cortex, plotted is the firing rate over time in $\mathrm{Hz}$. Vertical lines mark the moment the plate was moved upwards and started touching the finger or downwards, when the plate no longer touched the finger. The activity peaked at three time points for most conditions, when the plate first touched the finger, when it started moving and it left the finger again

A second example is given in figure 3.36, which shows a unit with very strong and significant differences between plate structures and platform movement directions. When the plate first touched the finger, differences between movement directions were already visible, with plates moving rightward eliciting a stronger response. When movement started, activity increase strongly depended on plate movement direction. Plate structures had a smaller influence. A small increase in activity could also be seen once the plates lost contact to the finger. 


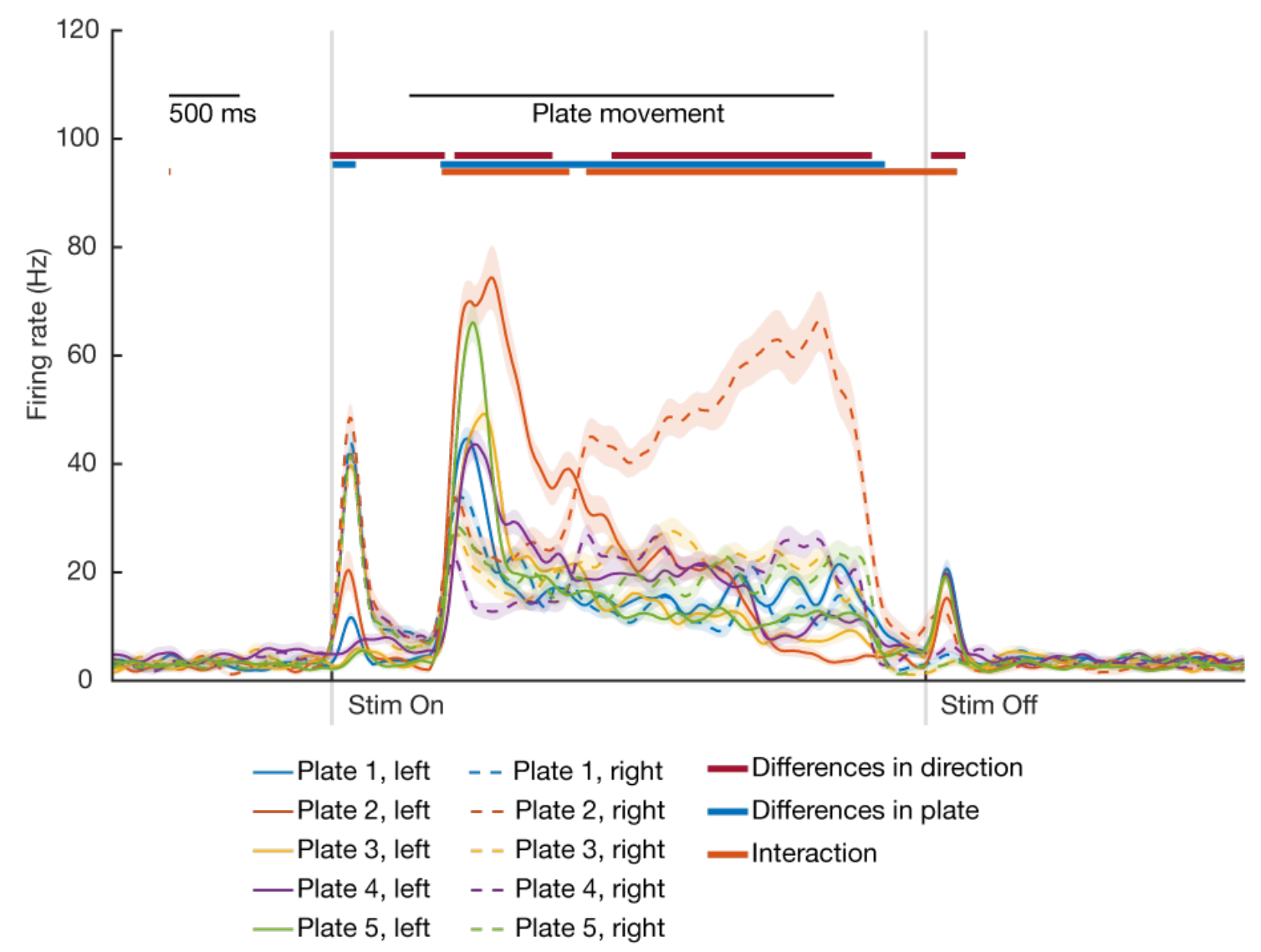

Figure 3.36: Firing rate of an $\mathrm{S} 1$ unit, showing significant differences in activity between conditions. A unit from primary somatosensory cortex, plotted is the firing rate over time in $\mathrm{Hz}$. Vertical lines mark the moment the plate was moved upwards and started touching the finger or downwards, when the plate no longer touched the finger. This unit showed significant differences between movement directions of the plate and plate structures.

\subsubsection{Population analysis revealed tactile tuning in S1 and $\mathrm{M} 1$ and no tuning in AIP and F5}

For this task population activity was investigated by looking at the number of significantly tuned units for all neurons recorded from an area. Two analyses were performed, one to see if significant differences between plate structures occurred and the other to see if there were differences for different movement directions. Figure 3.37 shows two examples for tuning curves for plate differences, recorded on two different days. The first recording was conducted early after implantation (January 2018), the second recording eight months later (September 2018). Early after implantation, a strong pattern, corresponding to the task, was visible in $\mathrm{S1}$, with a smaller change in significantly tuned units being visible in $\mathrm{M} 1$ and less changes being visible in F5 and AIP. In S1, an increase in the number of tuned units that corresponded to the moment the plates touched the finger, started moving and left the finger were visible, with smaller increases during movement. 

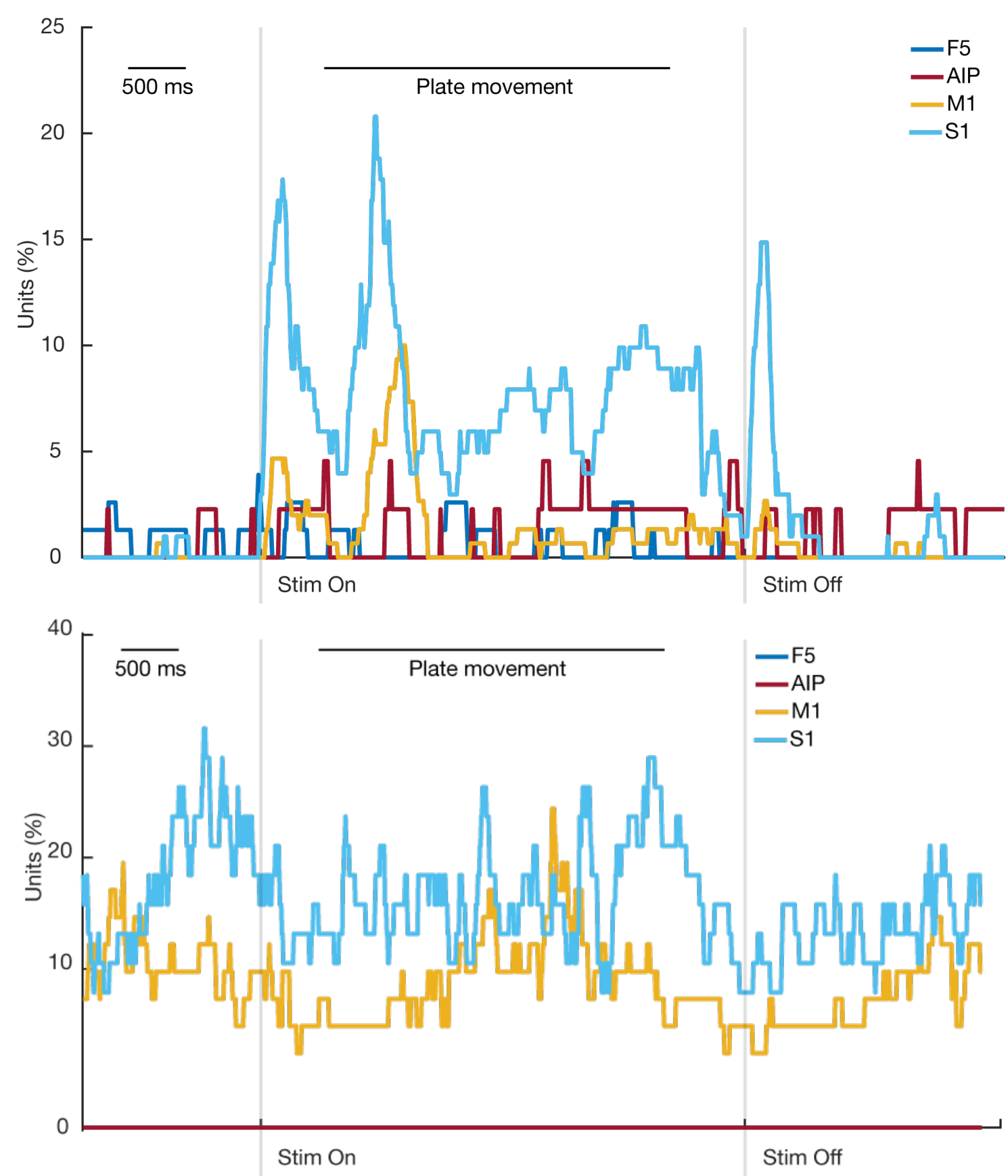

Figure 3.37: Number of significant units at each timepoint for passive stimulation task (factor plate structures). Number of significant units (ANOVA, factor plates) for each timepoint of the passive stimulation task. Vertical lines mark the moment the plates touched the finger and lost contact, respectively. Early after implantation (top), a clear pattern was visible for area S1, where an increased number of tuned units was found after the plate touched the finger, started moving and when it left the finger. The same pattern was present in M1, albeit weaker. Approximately eight month after the first recording (bottom panel), the pattern was gone for all areas.

In M1, an increased number of tuned units was visible after the plate touched the finger and started moving. For the second recording, all patterns disappeared. Overall, the recording seems to be comparatively noisy, since significant differences can be seen 
even before and after any stimulation happens and no correlation between task parameters (such as plate movement) could be seen. Due to these severe differences between recording sessions, no average population tuning was calculated.
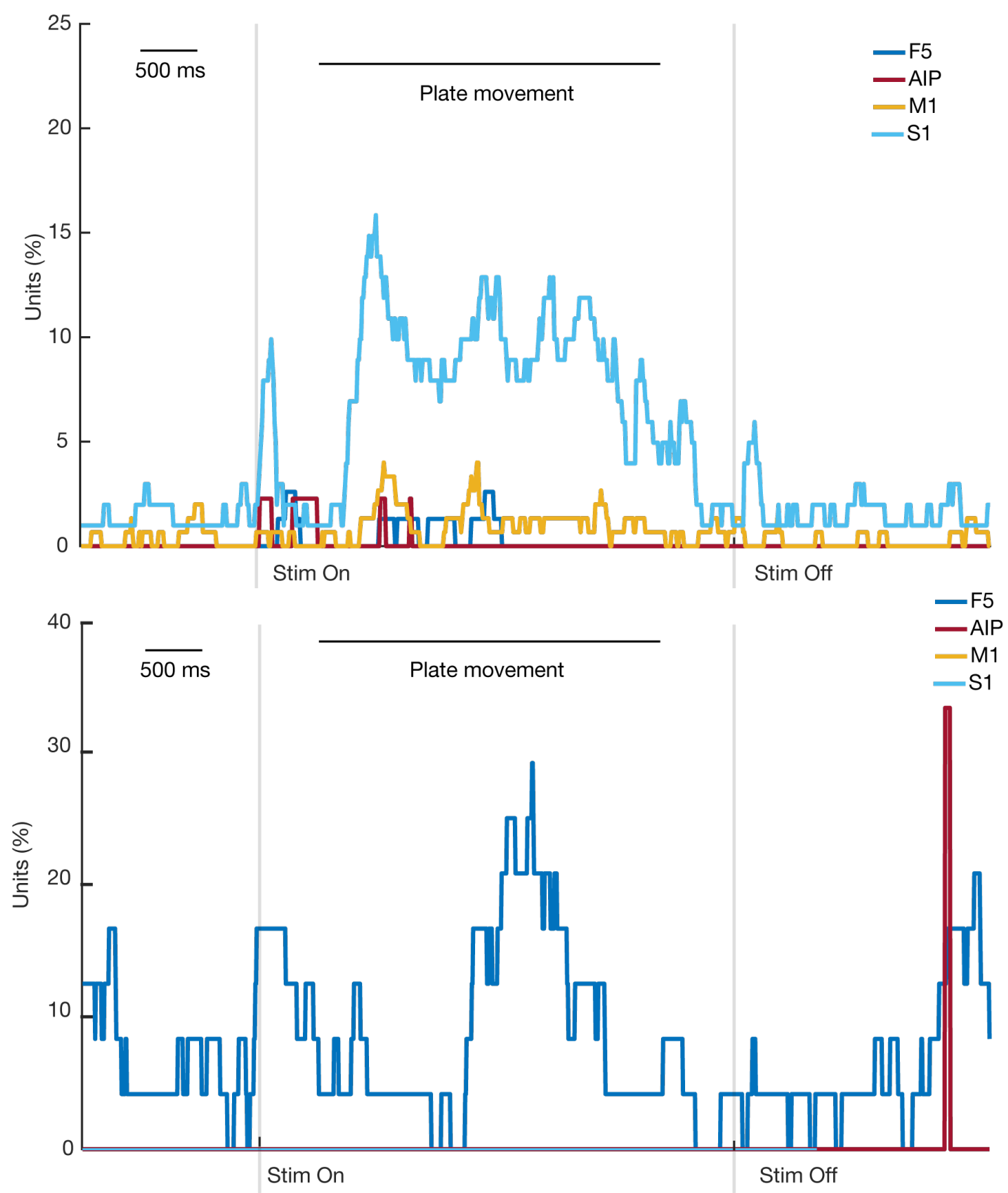

Figure 3.38: Number of significant units at each time point for passive stimulation task (factor plate movement direction). Number of significant units (ANOVA, factor plate movement direction) for each time point of the passive stimulation task. Vertical lines mark the moment the plates touched the finger and lost contact, respectively. Early after implantation (top), a clear pattern was visible for area S1, where an increased number of tuned units could be found after the plate touched the finger, started moving, during movement and when it left it. Approximately eight month after implantation (right), the pattern was gone for all areas. 
Figure 3.38 shows the number of significantly tuned units over time in percent for the plate movement direction. Again, the first plot shows an recording shortly after implantation, while the second one corresponds to a recording approximately eight month later. Similar to the results for the differences in plates, a pattern could be seen for the early recording but not for the later one. Again, during the earlier recording, tuning that corresponded to the moment the plate touched the finger, start of movement and the moment the plates left the finger could be seen in $\mathrm{S1}$, with some tuning during movement of the plate. M1 had a small increase (up to around 4\%) shortly after plate movement started.

Interestingly, this strong change in the overall pattern can only be observed in the passive stimulation experiment and not the active grasping experiment, where patterns are still present even in later (not shown) sessions. 


\section{Chapter 4}

\section{Discussion}

Our hands provide important tools for interactions with our surroundings. Using suitable grasps to pick up objects is important, not only to get a firm grip on something but also in order to avoid destroying it either by dropping or by squishing it (Napier 1962; Schaffelhofer et al. 2015; Smeets and Brenner 1999). In a related study, Schaffelhofer et al. investigated how visual object information is used for grasp planning in three different brain areas, the anterior intraparietal cortex, the ventral premotor area F5 and the primary motor cortex (Schaffelhofer and Scherberger 2016). Recordings from these three areas, that are part of the fronto-parietal grasping circuit (see section 1.1), revealed an interaction where visual object information is passed through the anterior intraparietal cortex towards planning areas such as the premotor cortex and result in movements after being processed in the primary motor cortex. However, Schaffelhofer et al. only investigated visual information and not how different senses might contribute to this circuit, where not only visual but also somatosensory information (such as the sense of touch or proprioception) play a role. In this project, I furthered our understanding of how the brain plans and generates movements, especially when not vision but the sense of touch lead our movements. I recorded data from an animal that had not only implants in the three areas of the fronto-parietal grasping circuit, but also in the primary somatosensory cortex (see section 2.2). While past studies could show that the primary somatosensory cortex plays a big role in representing the sense of touch in our brain (Delhaye et al. 2018; Penfield and Boldrey 1937), it was less clear how exactly touch is encoded in the three other brain areas, even when the animal is just passively touching objects. Furthermore, it is unknown how grasp planning differs when comparing visual and tactile inputs. 


\subsection{Visual and tactile object recognition}

When we attempt to grasp objects that lie in front of us, different senses can help to find the best strategy. Vision gives us a first estimate about size, structures or weight (often based on past experience), all factors that can influence the required hand shapes or used force (Breveglieri et al. 2017; Schaffelhofer 2014). Our sense of touch on the other hand, helps to understand the texture of objects and can also help to determine size and shape of objects that are out of view (Amedi et al. 2010; Delhaye et al. 2018; Lieber and Bensmaia 2019). The goal of this thesis was to determine how visual and tactile information influence grasp planning, when an animal only has one type of information available. To this end a monkey was trained to lift objects that he either saw or touched beforehand, as described in section 2.5 .

\subsubsection{The animal uses cue information to determine the right grasp type}

At the start of the project, one main concern was that the monkey might not use sensory information to determine the right grasp type. When we see objects in front of us, we almost automatically recognise its shape, colour, texture and other important features (Logothetis and Sheinberg 1996; Taira et al. 1990). This needs no real conscious effort and therefore is very different from tactually exploring an object. For tactile exploration, active movements need to be executed. Remembering the shape and structure of parts we just touched but moved away from is also a more active effort and might even require training (Behrmann and Ewell 2003). This might be especially true for an animal that just wants its reward as quickly as possible and therefore is more likely to try out strategies to explore an object as quickly as possible and checks whether he actually needs to remember the object in front of him or can forget about it immediately. A recent study in humans by Camponogara and Volcic 2019 even showed that human participants used different grasping strategies when they saw an object, compared to holding a copy of it in their non-grasping hand and using it to gain haptic information about the object. While the task in my project was different (the animal reached out with his grasping hand and felt the exact object it would lift), it was not clear whether the monkey might also use different grasps in both conditions. In order to encourage the animal to tactually explore the object and remember its shape, lifting it during the tactile cue epoch became an requirement. This meant the animal definitively made contact with the object and held it in his whole hand (in order to grasp, hold and lift). A second strategy was the restriction of movement times during the grasp epoch, which meant that the animal had no time to readjust its hand position if he tried to guess the objects and guessed wrong.

Looking back at figures 3.3 and 3.4, panel two and three of each figure, the animal was 
moving (and reacting) quicker during grasp epoch, compared to the cue epoch during which tactile exploration happened. Furthermore, the distribution of reaction and movement times in the first panel (lifting after visual cue) and the third panel (lifting after tactile cue) look quite similar. This gave a first hint that the animal actually recognises the object during tactile cue epoch and remembers it to perform an efficient and fast grasp during grasping. If the animal had to re-explore the object during the grasping epoch, movement and reaction times would be more similar to the tactile cue epoch and therefore slower than for visually guided grasps. As can be seen in section 3.3.1, this was not the case. Furthermore, visual analysis of video and hand kinematic recordings (as described in section 3.3) confirmed that the animal performed clearer and more direct grasps during both grasping epochs, as compared to tactile exploration. During tactile exploration, it was obvious in some trials that the animal either did not know which object was in front of him or guessed wrong, since he visually hit the object with his hand, instead of

directly grasping it. More so, in some trials the animal did grasp the object in front of him but the hand shape made it impossible to lift the object, requiring him to readjust his hand shape, as illustrated in section 1.1, figure 3.1. This also explains why movement times during tactile exploration where higher. When the animal does not know which object he has to grasp, he needs some time to explore the object and decide on the right grasp. As soon as the object is known (either from seeing it or from having it touched before), movement time becomes faster since no readjustment is needed, as illustrated in section 1.1, figure 3.2).

\subsubsection{Visual and tactile trials elicit a slightly different memory activity}

Our brains are driven by changes in electric activity (Fritsch and Hitzig 1870). These activity changes can be measured with electrodes, as described in section 1.2, and offer us a window into the computations our brain does during certain tasks. This can help to understand how the brain works. By looking at the changes in activity over time, we can get a first idea of the wide variety of pattern that drive behaviour and perception. After recording and preprocessing the electric raw data from the implants of a monkey, I plotted the average firing rate over time, as described in section 3.3.2. This type of analysis helps to get a first, very rough view into the wide variety of firing patterns that neurons can show during behaviour. Due to the high number of units recorded each day, only a small number can be discussed in order to give an overview into which types of patterns were revealed by the PSTHs and what those patterns tell us. First, many neurons show activity patterns that somewhat match known "textbook knowledge", such as M1 or F5 neurons reacting to movements (Fluet et al. 2010; Murata et al. 1997; Raos et al. 2006; Umilta et al. 2007). Two such motor driven neurons can be found in figures 3.5 and 3.7, 
but the activity of individual neurons can stray quite far from these patterns and some neurons might react to completely different cues. An example for this can be seen in figure 3.8, which shows a motor cortex unit reacting to visual information, alongside motor activity. While this pattern of a motor cortex unit reacting to visual information might seem surprising at first, other studies have also described M1 units that respond to sensory input (Hatsopoulos and Suminski 2011; Suminski et al. 2009). While those studies often investigated reactions to visual movement stimuli, other studies could also show that simply imagining and preparing movements can drive motor cortex activity (Miller et al. 2010; Riehle and Requin 1989). Whether or not the animal does imagine movements after seeing the object or not can not be answered. Hand kinematic recordings showed that the animal does not preshape his hand during or after cue presentation and leaves it in a relaxed position on the handrest button, so preshaping can be eliminated as a possible source of visual activity in motor cortex. The activity observed in some motor cortex units can even go completely against typical and expected patterns. For example, the unit shown in figure 3.9 shows a decrease in activity during tactile exploration, even though movement is involved in this epoch. During grasping period however, a slight increase can be seen for most conditions. More importantly, even though tactile input seems to decrease the firing rate of this unit, a strong increase in activity during memory period can be seen, hinting at some kind of memory or movement preparation sensitivity. This activity greatly differs between visual and tactile trials. Still, when looking at the entire population recorded, most of these expected patterns become apparent, while the few neurons reacting to other input become almost invisible, showing how even with a few "stray neurons" an area can still show tuning towards a certain type of information or input.

In an attempt to further see how well the recorded populations of whole areas encode sensory information, a receiver operating characteristic analysis and decoding analysis were performed (sections 2.7.5 and 2.7.6). The ROC analysis was applied to each unit separately. Afterwards, the distribution of the resulting ROC scores was plotted for each area, during five epochs: baseline, cue, early memory, late memory and grasping epochs, where baseline and cue mainly served as a control for this measure. The resulting pattern for all four areas was quite similar: Overall, the ROC analysis was able to differentiate between visual and tactile conditions on some but not all units. During early memory, mean ROC score for F5, M1 and S1 fell between 0.67 and 0.74 . This number decreases heavily during late memory and grasping, where no units achieve a score above 0.9 , indicating discrimination between sensory conditions became much harder. The mean scores for all areas were close to chance level during both epochs. This is not totally unexpected. During early memory period, some remaining activity from the different cue epochs might still be found in some units. For example in the M1 example unit in figure 3.7, a decrease 
in activity towards the end of the tactile cue period is visible, but the unit finishes its activity decrease only after memory period starts, even though the movement is finished already. However, this supposed "remaining movement activity" only lasts around 100ms. Afterwards, it very shortly remains at an activity level similar to visual trials, before rising again. This means that activity between visual and tactile trials is indeed different during the early memory period, at least in this example unit. In the other two examples (figures 3.8 and 3.9) activity between visual and tactile trials differs even more severely. A more important measurement for this supposedly difference between visual and tactile trials in the early memory period can be found by looking at the population tuning curve in figure 3.15 . Here, between $40 \%$ and $60 \%$ of units are tuned during early memory period, again not only lasting longer than the observed remaining motor activity in figure 3.7, but showing a significant difference between both conditions during the whole period . Similarly, decoding using LDA during the same period was quite successful, reaching an accuracy above $70 \%$ for all three areas (see figure 3.27). In conclusion, all three analyses (ROC analysis, population tuning and decoding) revealed a significant difference between visual and tactile trials during early memory, up to $500 \mathrm{~ms}$ after the cue period and before the animal had to execute the grasp movement. More interestingly, these differences remained visible even during late memory, albeit less pronounced. When looking at the whole population of each area, some units still showed significant tuning during late memory, although considerately fewer units than during early memory. For $\mathrm{S1}$, around $7 \%$ of units showed significant tuning, for $\mathrm{F} 5$ and $\mathrm{M} 1$ around $15 \%$ of units were tuned. While this number appears to be quite low, decoding for F5 and M1 was still possible above chance level (ranging between $90 \%$ and $65 \%$ for $\mathrm{M} 1$ and $85 \%$ and $60 \%$ for F5). Overall, decoding accuracy decreased during late memory. For S1, while decoding started at around $75 \%$, it decreased to almost $50 \%$ shortly before movement starts. Overall that is not surprising, as one would expect that the way the object was recognized should no longer matter, once movement starts and only the needed grasp type should be important. Therefore it is actually surprising that especially M1 and F5 still showed decoding above chance level even during the actual grasp movement execution, even though only slightly (around 60\% for both areas).

So in conclusion, differences in the memory period of the active grasping task are present between visual and tactile trials. When starting this project our first hypothesis was that no big differences in motor related areas should be present, since the outcome is the same even if the way that leads to the grasp might be different with involvement of different brain areas. Especially the artificial delay in the active grasping task (compared to natural grasping where delays, especially in accordance to a visual cue, are almost non-existent) should give the brain enough time to merge grasping plans or adjust it. As described in section 3.3, video and hand kinematic recordings showed that the grasp the 
animal chooses to lift the object is very similar between visual and tactile trials. Indeed, during grasping most analyses showed close to no difference in activity and tuning during grasping period (sections 3.3.2 and 3.3.3), although these small difference seem to be big enough to allow decoding slightly above chance level (sections 3.3.4 and 3.3.5. Early memory activity possibly differs due to different cue periods where the animal either sits still and looks at an illuminated object or moves his hand over the object without seeing anything. Still, these different activities drop quite fast and usually seem to result in memory patters that represent objects. The assumption was that during late memory, these differences should no longer matter. The memory period lasts 1 to $1.5 \mathrm{~s}$ (random length), therefore the animal is sitting for at least 1s without moving, visual input or tactile input from the object (the handrest button provides tactile input but nothing relevant to the task). At this point any brain activity should most likely be dedicated to memorizing the object and preparing the movement and not how the object information was delivered, especially considering that the animal is well trained in identifying the object and was not presented unknown ones. One reason why the memory activity might differ between both sensory conditions could be the involvement of different brain areas.

\subsubsection{Differences in the encoding of objects}

In addition to looking at sensory modalities, I also investigated how different objects and hand shapes were encoded in these brain areas. For grasping the specified six objects, the animal needed to apply three different grip types. One similar to a precision grip to lift the cube and the sphere, one grip from the side to grasp the side of the two rings and one similar to a power grip, where the bar and the block had to be grasped with the full hand. The objects were designed in pairs to test whether maybe hand shape and not object identity was encoded in some areas, similar to the findings of Schaffelhofer et al. 2015. There, F5 showed some tuning towards grip type but not towards objects that looked similar but were grasped differently (for example a small and a big sphere). In figures 3.13 and 3.14, tuning is plotted over time for each population of recorded neurons, separated by area. The first plot shows a tuning curve for visual trials, the second one shows a tuning curve for tactile trials. During visual trials, F5 and M1 showed an increase in activity during visual presentation of the objects (cue period). Since the animal was not moving during cue epoch, this activity might come from movement preparation or some kind of imagined movements, which are known to be able to elicit activity in the premotor and motor cortex (Decety et al. 1994; Hatsopoulos and Suminski 2011; Stephan et al. 1995). During early memory, a memory effect was visible in F5, a sustained, increased activity starting at the end of cue presentation and continuing until shortly before the movement started. For M1, after the small, initial increase during cue, tuning only increased during 
the late memory period and rose shortly before movement started, as would be expected for motor cortex. S1 showed almost no tuning during the memory period, with only a small rise above $5 \%$ shortly before movement started. During neither of these periods any task related tactile information is present, so any significant differences would be unexpected. Tuning increased during the grasping period, when the object was touched and tactile input was present and very different for the six objects. During tactile trials, all areas showed more tuning. This is expected during the cue period since here the animal has to move (so an increase in activity of F5 and M1 would be expected) and tactile input is present. Interestingly, for the first approximately $200 \mathrm{~ms}$, no significant tuning was visible, which would account for reaction time and possibly also the start of the reach, where no difference in movement would be expected and no tactile input (but possibly proprioceptive) input is present. During early memory, all three areas showed a memory effect, although for F5 tuning was weaker than during visual trials, while the tuning in M1 was comparable although less stable. At the start of the late memory epoch, tuning in $\mathrm{S} 1$ and $\mathrm{M} 1$ was very low but rising from there and peaking during grasping. Tuning in F5 started out higher during late memory and also rises from there.

In summary, during visual trials tuning for different objects is present in F5 during the whole trial, while in $\mathrm{M} 1$ and $\mathrm{S} 1$ tuning rises during late memory and peaks during grasping, when contact with the different objects is made. During tactile trials, a similar pattern can be seen. This suggests that independent of sensory condition, decoding for objects or grip type should be low during early and late memory and far better during grasping. When looking at the decoding accuracy for different objects (figure 3.26), a similar pattern could indeed be found. Since six objects were used, chance level for decoding would be $16,7 \%$. Similar to the results from the population tuning analysis, decoding accuracy during early memory was above chance for M1 and F5 and rose from there, until it peaked shortly after movement started. Shortly before the movement started, a go cue appeared, instructing the animal to lift the object. When the go cue appeared, the animal should initiate movement after some reaction time (for an overview of reaction times during the trials see figure 3.3), meaning at this point in the late memory epoch other information was already available to the animal and its no longer simply waiting for the cue to appear. This might explain why accuracy already rises before movement starts. Since the reaction time differs, the exact moment of the go cue can not be averaged without realigning the plot, but average reaction time was $259 \mathrm{~ms}$ during visual and $256 \mathrm{~ms}$ during tactile trials. Anticipating of movement might also explain this early and steady increase in decoding accuracy. Due to the low recording quality and number of units, decoding accuracy in AIP remains close to chance level most of the time and only rises to around $30 \%$ during grasping.

Overall, objects could be decoded above chance level for most of the memory and grasp- 
ing epochs in F5, M1 and S1. Interestingly, the accuracy was lowest shortly after the object was seen or touched, during early memory period. At this moment, a higher accuracy might have been expected, since the object identity should be fresh in the mind of the animal. Instead, the accuracy only increases over time, meaning decoding during late memory, shortly before the grasp happens, was better than during early memory in all four areas (although the accuracy of decoding based on AIP was generally low). It is possible that during the early memory period, the object information still is processed in other brain areas where I did not record from and only over time gets pushed into the recorded areas. It is also possible that not so much the object information but movement information is encoded and therefore the brain needs some time to process the freshly acquired object information to produce a meaningful movement. In this case, a lower accuracy would have been expected, since only three grasps are needed to grasp all six objects, which should lead to confusion between both objects of a pair. But of course the grasps might not be perfectly identical. On a observable level the movements and the general grasp (such as where he positions his fingers) was similar, but due to the different shape of the objects small adjustments might be needed. In each object pair one object has a round surface while the other has edges. When grasping the edged objects, the animal might aim to avoid applying force directly on the edges and therefore might adjust his hand position differently when compared to round objects. On the other hand, while the objects are generally not slippery, the round surface might encourage a more firm grip to avoid possible slippage, compared to grasping the flat surfaces of the edged objects.

\subsection{Passive finger stimulation}

Our sense of touch can be an important factor for determining the structures and textures of objects. While the active grasping task (section 2.5) contains elements where tactile information is present (such as tactile exploration of the object or simply while the object is lifted and held up), tactile information never occurs alone. When the animal is instructed to tactually explore the object in front of him, he has to start moving to reach it and moves his hand around the object, in order to determine its shape. This means that movements and a change in proprioception is always coupled to the occurrence of tactile information, making it hard to see how tactile information is represented in all four areas alone. In order to look at the encoding of tactile input more specifically, a passive finger stimulation task (section 2.6) was used. It is important to note that no direct comparison between the active grasping and the passive stimulation task is possible, since the tactile input is quite different. During active grasping, differently shaped objects (section 2.5.2) made from the same material have to be grasped. This means that the overall structure is always the same and only the shapes differ. The monkey was also allowed to touch 
the objects with his whole hand. On the passive stimulation task, however, plates with different structures (section 2.6.2) were presented passively, i.e. without any movement of the monkey, to a single finger. Still, this task could give some insights in how tactile information is encoded in the four brain areas.

\subsubsection{During passive finger stimulation, tactile stimulation elicits responses in $\mathrm{S} 1$ and M1, but not in AIP and F5}

In the passive finger stimulation experiment, the animal was not only instructed to hold still but his finger was fixed to the setup, so that no movement was needed in order to receive his reward. Furthermore, not only different plate structures were used, but the presentation direction of the stimulus changed in order to see whether or not presentation direction would have any influence on neuronal activity. Afterwards, the firing rate of units from each electrodes were plotted (section 3.4.1) to see whether or not task dependent activity could be found. As expected for units from the primary somatosensory cortex, task dependent activity changes were easily found during the passive stimulation experiment, where changes in tactile stimulation are happening. Usually, unit activity changed the moment the plate started or stopped touching the finger as well as when movements of the platform below the finger started or stopped. Anatomically, the primary motor cortex sits opposite to the primary somatosensory cortex, which both lie directly at the central sulcus in mammals (Fritsch and Hitzig 1870; Penfield and Boldrey 1937). It was therefore not unexpected that some M1 units also showed task dependent activity changes during the presence of tactile information. Indeed, past studies also found activity changes driven by tactile input in the primary motor cortex and could prove that it is not only generating movements but also part of a circuit that processes tactile and other sensory information (Fetz et al. 1980; Hatsopoulos and Suminski 2011; Schroeder et al. 2017), making it rather unlikely that the activity changes observed here stem from finger movements of the animal. Moreover, the platform maintained a consistent force during stimulus presentation. This means that whenever the animal pushed against the plate (which was already hard since the finger was fixed) his movements were countered by a change in executed force, to keep the overall force constant. While some activity changes in single trials might stem from movements of the finger, those would be recorded by the platform and could be detected. During most trials, I could confirm that the animal did indeed not move his finger against the plate.

In AIP and F5, units that responded to the task were sparse. While the first recording of the passive stimulation task did result in the highest number of AIP units of all recordings (section 3.1, table 3.1) most units showed activity patterns, that were not correlated with the task, similar to figure 3.31. The same was true for area F5. A few hand picked units 
showed reactions to tactile input but were not representative for the whole population. Furthermore, those changes in activity usually occurred during certain task epochs and not in correspondence to a certain type of plate or movement direction. To get a better idea how the investigated areas encode tactile information, the number of significantly tuned neurons over time was computed. During the first recording of the passive stimulation task (figures 3.37 and 3.38), somatosensory cortex showed tuning towards tactile inputs and changes in input for different plate structures and movement directions. This was no surprise, since it is already known that the somatosensory cortex reacts to tactile stimulation (Hyvärinen and Poranen 1978; Lieber and Bensmaia 2019; Weber et al. 2013). The first peak occurred the moment the plate started touching the finger, resulting in a clear increase in activity in most units and therefore a significant change in the number of units in the population tuning curve. Afterwards, tuning and unit activity went down, most likely since the plate remained still for a few milliseconds, to ensure that the end position has successfully been reached. Once the plate started moving leftwards or rightwards, activity (and therefore tuning) increased again. At this moment, the different ridges on the plate started to move on the finger, eliciting responses. Individual ridges did not cause any individual obvious activity changes, overall, more of an ongoing activity increase (figure 3.35. One reason for this could be the comparatively high stimulus presentation speed of $25 \mathrm{~mm} / \mathrm{s}$. For $\mathrm{F} 5$, these results were to be expected since the animal is not moving around and is instead encouraged to hold still. Since F5 is mostly known to be modulated by movements, a lack of modulation in the absence of movements was to be expected. A similar conclusion can be found for AIP, a more visually driven area. Since this task does not contain any visual elements and the animal sits in the dark the whole time, no changes in activity were to be expected.

\subsection{Tactile input in the different brain areas}

\subsubsection{Motor cortex responded to tactile input as well as active move- ments of the animal}

In the primary motor cortex, the results from both experiments were mostly as expected. There always was a strong increase in activity whenever the animal moved and a weaker one when passive stimulation was applied. While the motor cortex is mostly known for generating movement, past studies could show that it also reacts to different sensory information (see section 1.1.4), therefore the increase in activity during passive tactile finger stimulation was not unexpected (Clark and Ward 1948; Hatsopoulos and Suminski 2011; Suminski et al. 2009). More so, motor cortex sits directly opposite of the so- 
matosensory cortex, with direct connections between both areas (Penfield and Boldrey 1937). Still, even though most results were expected, the difference in activity between visual and tactile trials was remarkable. The starting hypothesis was that the motor cortex is more "simple" and might not care about where object information comes from. It might just care about what to do with the information and how to generate a meaningful movement. Therefore the differences in activity and tuning shortly before the movement starts were surprising, hinting at a more complex way the brain handles the generation of movements, even if the motor output is the same.

\subsubsection{In both tasks the somatosensory cortex reacted to tactile stimuli}

Opposite to motor cortex sits the primary somatosensory cortex (Penfield and Boldrey 1937). Again, most results matched expectations. The somatosensory cortex responded to tactile inputs, no matter if those were presented passively or actively (Delhaye et al. 2018; Woolsey and Fairman 1946). Although data was recorded from area 3b, which is mainly representing cutaneous tactile stimuli, some neurons might still encode proprioceptive information (Bensmaia and Tillery 2014; Delhaye et al. 2018; Iwamura et al. 1983). In the passive stimulation task it is clear that all task relevant information comes from the sense of touch, since the posture of the animal is not changing. In the active grasping task, this separation is harder to make, since all task epochs containing tactile object information were also epochs when the animal is moving actively. Still, going by past studies and the results of the passive stimulation task, it is more likely that most neurons increased activity due to the presence of tactile information. In the tuning curves, a small increase in tuning can also be seen at the presentation of the visual cue, which again matches the results of different studies (see section 1.1.2). In S1 the differences in memory activity are more obvious. While both trials contain tactile information, during visual trials this information is only available once the object is already lifted. During the cue epoch, when the object is illuminated, no tactile information is available to the animal. Therefore it is less surprising that almost no tuning could be found during this epoch and no memory activity is visible during early memory. Interestingly some tuning is present during late memory (figure 3.13). Where this increase in tuning comes from is unclear. It could be that the animal is beginning to image the object (although this activity would be expected sooner, when the animal could see the object and might try to remember how it felt). Since the animal knew that a grasp has to follow after the memory period, it could also be some kind of anticipation signal, where the animal either anticipates the start of the movement while he waits for the go cue to appear, or he already anticipates how the object will feel in his hand.

More surprising was the vanishing tuning in the passive stimulation task during later 
recording session of the passive stimulation experiment. During early recordings, some clear modulation of this area with the presence of and changes in tactile stimulation was found, just as one would expect. During later recordings (not shown) this effect got weaker and completely disappeared in the last recording session of this experiment. Interestingly, no similar effect was found in the active grasping task. While some influence of recording quality (such as the number of recorded units) could be observed, the observed effects never disappeared completely. An important difference between both tasks is the skin area where stimulation was applied to. While both tasks indicate that the hand area of $\mathrm{S} 1$ was successfully implanted, only a single finger was stimulated in the passive stimulation task, while the whole hand was used in the active grasping task. Overall, a decrease in the number of recorded units could be observed in most areas. Past studies showed that the neurons an electrode records from can change quite quickly from session to session or even day to day, with some neurons being present only during a single recording session, even with chronically implanted electrodes. It is possible that the units which showed tuning towards the task simply were no longer recorded from, either due to small shifts in electrode position or other biological processes such as scarring (see section 1.2). In future experiments, one could map the array (by poking different locations of the hand of the monkey and listing to changes in activity) on different recording sessions to be able to observe this better.

\subsubsection{The anterior intraparietal area shows no modulation to tactile in- put}

Results for AIP were less conclusive due to the reduced number of recorded units and low recording quality of this area. Interestingly, even though AIP is known as a more visual area, it does respond to movements and tactile information, showing significant tuning during the cue epoch for both conditions. During tactile trials, no memory effect could be observed. This would be expected for a more visually driven area in trial conditions when no visual information is present. This also matches the results of the passive stimulation task where no tuning could be found in AIP (neither in early nor in later recordings). This means that the increase in tuning during the tactile exploration is most likely also a modulation by movement and not by tactile input, matching the increase in tuning during grasping. 


\subsubsection{Premotor cortex area F5 responded to movements of the animal but not passive tactile inputs}

Similar to the results of $\mathrm{M} 1$ and $\mathrm{S1}$, results for $\mathrm{F} 5$ also matched literature. This area is known to be involved in the planning of movements, therefore it was not surprising to see that a lot of its activity changes where modulated by movements, such as tactile exploration or lifting of the object (Churchland et al. 2006). There was no significant tuning during the first recordings of the passive stimulation task, which again would be expected since the animal was not actively moving. Interestingly, during later recordings more significant tuning was present but this was not corresponding to any task parameters (such as start of tactile stimulation). Since at this point the implants of the animal were over a year old it could be that the overall recording quality was too low and too noisy to record any meaningful modulations. Again, this effect was not visible in the recordings of the active grasping task, making it hard to say why exactly this pattern became apparent. Since significant differences between plate structures and movement directions are visible before and after the plate moved or touched the finger it is very likely that this pattern is the result of some random noise and not correlated with anything task related. During the active grasping task, however, F5 more clearly responds to movements and usually shows a strong memory effect during early and late memory epoch. While on first sight this memory effect looks similar during tactile and visual trials, I was able to show that there is still a significant difference between visual and tactile trials that can also be used to decode the sensory condition from trials. This difference is even present during the start of the grasping period but decreases as movement goes on. The size of this difference was quite surprising given the hypothesis was that this planning area might care less about the sensory condition of a trial. The expected role of F5 is to integrate the information from different senses (most likely during cue and early memory period) to plan a meaningful movement. During late memory, when the grasp will happen soon, the sensory condition should matter less but could still be present to some extent. 


\section{Chapter 5}

\section{Conclusion}

In this project, two different experiments were used to find out how visual and tactile input influences the fronto-parietal grasping network. In the delayed-grasping experiment, the influence of visual and tactile information on grasp planning and execution was investigated. Two monkeys were trained to grasp objects that they either saw or touched beforehand. So far, data from one animal was recorded in four different brain areas: Primary motor cortex, premotor cortex area F5, anterior intraparietal area AIP and primary somatosensory cortex. In the active grasping experiment I focused especially on differences in the memory period, where the animal is assumed to prepare the actual movement: The animal already got the cue information and just waited for the go cue to finally lift the object and gather his reward. I found small differences between memory activity in visual and tactile trials that were not explainable by different task parameters. In a population analysis I demonstrated that these small differences are significant. Futhermore, they could be used by an LDA decoder to successfully predict task conditions from neuronal activity.

In a second experiment I investigated, how tactile information is processed in the frontoparietal grasping network and the primary somatosensory cortex. Small plates with different stimuli were passively moved below the middle finger of the monkey. Results showed the expected activity changed in the somatosensory cortex and the motor cortex during tactile stimulation. However, these effects vanished over recording sessions, until no task related effect could be found in any areas. While the second, passive stimulation task yielded no conclusive results, results from the active grasping task showed that the influence of different senses should not be neglected when trying to decode neural activity. This could be especially relevant for the control of neural prosthesis, where one tries to decode brain activity to control a robotic arm. If activity is not consistent between objects that were seen versus touched, more considerations about the origin of object information would be needed. 
In conclusion, the brain processes visual and tactile information differently, as expected. During active touch, all four areas react to the cue (and possibly the movement), while less changes in activity are visible during passively observing the object (in the active grasping task) or passively being touched by a stimulus on a single finger (in the passive stimulation task). During the memory period of the grasping task, the brain processes this information and needs to generate a grasping plan that will lead to a meaningful movement. While one might expect that the generation for this grasping plan is similar under all conditions, I could demonstrate that this is not the case. Significant changes appeared when comparing activity and tuning patterns between conditions. This is especially true during early memory, shortly after all movement, tactile input and visual inputs (depending on the trial) vanished. While some remaining activity had to be expected while the animal returns to a resting state, the significant differences between the two sensory conditions lasted longer then explainable by remaining activity. Even shortly before the movement starts, between 1000 ms and 1500 ms after the cue period ended, a decoder was able to differentiate between visual and tactile trials.

Overall all four areas showed differences in their neuronal spiking activity when exposed to visual and tactile information, indicating the need to consider sensory conditions when one aspires to record and decode from these areas but also when planning tasks that involve these areas. More importantly, differences between a task with and without grasp intentions could be found. While both task designs differ too greatly for an direct comparison (such as the usage of different textures), the results imply that especially F5 might not be modulated by tactile information that is passively delivered but only when it becomes relevant later (as seen in an increase in neuronal activity or "memory effect" during the active grasping task), showing that not only the type of sensory information might be relevant but also the context. Results for M1 and S1 were more conclusive, since a clear task related modulation could be seen in both experiments, indicating that these areas are driven by tactile input. Again it could be shown that the source of object information is present in both areas even during the memory period of the active grasping task, meaning sensory input, especially if directly relevant to the task, should not be ignored. 


\section{Chapter 6}

\section{References}

\section{References}

Adewole, D. O., M. D. Serruya, J. P. Harris, J. C. Burrell, D. Petrov, H. I. Chen, J. A. Wolf, and D. K. Cullen (2016). "The evolution of neuroprosthetic interfaces". In: Critical Reviews in Biomedical Engineering 44.1-2.

Amedi, A., N. Raz, H. Azulay, R. Malach, and E. Zohary (2010). "Cortical activity during tactile exploration of objects in blind and sighted humans". In: Restorative neurology and neuroscience 28.2, pages 143-156.

Arbous, M. S., D. Grobbee, J. Van Kleef, J. De Lange, H. Spoormans, P. Touw, F. Werner, and A. E. E. Meursing (2001). "Mortality associated with anaesthesia: a qualitative analysis to identify risk factors". In: Anaesthesia 56.12, pages 1141-1153.

Asanuma, H. and K. Arissian (1984). "Experiments on functional role of peripheral input to motor cortex during voluntary movements in the monkey". In: Journal of neurophysiology 52.2, pages 212-227.

Astrand, E., C. Wardak, and S. Ben Hamed (2014). "Selective visual attention to drive cognitive brain-machine interfaces: from concepts to neurofeedback and rehabilitation applications". In: Frontiers in systems neuroscience 8, page 144.

Augurelle, A.-S., A. M. Smith, T. Lejeune, and J.-L. Thonnard (2003). "Importance of cutaneous feedback in maintaining a secure grip during manipulation of hand-held objects". In: Journal of Neurophysiology 89.2, pages 665-671.

Baker, K. C., M. Bloomsmith, K. Neu, C. Griffis, M. Maloney, B. Oettinger, V. A. Schoof, and M. Martinez (2009). "Positive reinforcement training moderates only high levels of abnormal behavior in singly housed rhesus macaques". In: Journal of Applied Animal Welfare Science 12.3, pages 236-252. 
Baker, K., M. Bloomsmith, K. Neu, C. Griffis, and M. Maloney (2010). "Positive reinforcement training as enrichment for singly housed rhesus macaques (Macaca mulatta)". In: Animal welfare (South Mimms, England) 19.3, page 307.

Barbas, H. and D. Pandya (1987). "Architecture and frontal cortical connections of the premotor cortex (area 6) in the rhesus monkey". In: Journal of comparative neurology 256.2, pages 211-228.

Barrese, J. C., N. Rao, K. Paroo, C. Triebwasser, C. Vargas-Irwin, L. Franquemont, and J. P. Donoghue (2013). "Failure mode analysis of silicon-based intracortical microelectrode arrays in non-human primates". In: Journal of neural engineering 10.6, page 066014.

Baumann, M. A., M.-C. Fluet, and H. Scherberger (2009). "Context-specific grasp movement representation in the macaque anterior intraparietal area". In: Journal of Neuroscience 29.20, pages 6436-6448.

Bautista, D. M., J. Siemens, J. M. Glazer, P. R. Tsuruda, A. I. Basbaum, C. L. Stucky, S.-E. Jordt, and D. Julius (2007). "The menthol receptor TRPM8 is the principal detector of environmental cold". In: Nature 448.7150, page 204.

Begliomini, C., T. De Sanctis, M. Marangon, V. Tarantino, L. Sartori, D. Miotto, R. Motta, R. Stramare, and U. Castiello (2014). "An investigation of the neural circuits underlying reaching and reach-to-grasp movements: from planning to execution". In: Frontiers in human neuroscience 8, page 676.

Behrmann, M. and C. Ewell (2003). "Expertise in tactile pattern recognition". In: Psychological Science 14.5, pages 480-492.

Bensmaia, S. and S. I. H. Tillery (2014). "Tactile feedback from the hand". In: The human hand as an inspiration for robot hand development. Springer, pages 143-157.

Berger, H. (1929). "Über das elektroenkephalogramm des menschen". In: Archiv für psychiatrie und nervenkrankheiten 87.1, pages 527-570.

Binkofski, F., G. Buccino, S. Posse, R. J. Seitz, G. Rizzolatti, and H.-J. Freund (1999). "A fronto-parietal circuit for object manipulation in man: evidence from an fMRI-study". In: European Journal of Neuroscience 11.9, pages 3276-3286.

Binkofski, F., C. Dohle, S. Posse, K. M. Stephan, H. Hefter, R. J. Seitz, and H.-J. Freund (1998). "Human anterior intraparietal area subserves prehension: a combined lesion and functional MRI activation study". In: Neurology 50.5, pages 1253-1259.

Bloomsmith, M., A. Stone, and G. Laule (1998). "Positive reinforcement training to enhance the voluntary movement of group-housed chimpanzees within their enclosures". In: Zoo Biology: Published in affiliation with the American Zoo and Aquarium Association 17.4, pages 333-341.

Borra, E., A. Belmalih, R. Calzavara, M. Gerbella, A. Murata, S. Rozzi, and G. Luppino (2007). "Cortical connections of the macaque anterior intraparietal (AIP) area". In: Cerebral Cortex 18.5, pages 1094-1111. 
Bowden, D. and M. Martin (2000). Primate Brain Maps: Structure of the Macaque Brain: A Laboratory Guide with Original Brain Sections, Printed Atlas and Electronic Templates for Data and Schematics.

Breveglieri, R., M. De Vitis, A. Bosco, C. Galletti, and P. Fattori (2017). "Interplay between grip and vision in the monkey medial parietal lobe". In: Cerebral Cortex 28.6, pages 2028-2042.

Brodmann, K. (1909). Vergleichende Lokalisationslehre der Grosshirnrinde in ihren Prinzipien dargestellt auf Grund des Zellenbaues. Barth.

Butterworth, S. et al. (1930). "On the theory of filter amplifiers". In: Wireless Engineer 7.6, pages 536-541.

Buzsáki, G., C. A. Anastassiou, and C. Koch (2012). "The origin of extracellular fields and currents-EEG, ECoG, LFP and spikes". In: Nature reviews neuroscience 13.6, page 407.

Camponogara, I. and R. Volcic (2019). "Grasping movements toward seen and handheld objects". In: Scientific reports 9.1, page 3665.

Castiello, U., K. Bennett, and C. Mucignat (1993). "The reach to grasp movement of blind subjects". In: Experimental brain research 96.1, pages 152-162.

Castiello, U. (2005). "The neuroscience of grasping". In: Nature Reviews Neuroscience 6.9, page 726 .

Chapin, J. K., K. A. Moxon, R. S. Markowitz, and M. A. Nicolelis (1999). "Real-time control of a robot arm using simultaneously recorded neurons in the motor cortex". In: Nature neuroscience 2.7, page 664 .

Chaure, F. J., H. G. Rey, and R. Quian Quiroga (2018). "A novel and fully automatic spikesorting implementation with variable number of features". In: Journal of neurophysiology 120.4, pages 1859-1871.

Chestek, C. A., V. Gilja, P. Nuyujukian, J. D. Foster, J. M. Fan, M. T. Kaufman, M. M. Churchland, Z. Rivera-Alvidrez, J. P. Cunningham, S. I. Ryu, et al. (2011). "Long-term stability of neural prosthetic control signals from silicon cortical arrays in rhesus macaque motor cortex". In: Journal of neural engineering 8.4, page 045005.

Churchland, M. M., M. Y. Byron, S. I. Ryu, G. Santhanam, and K. V. Shenoy (2006). "Neural variability in premotor cortex provides a signature of motor preparation". In: Journal of Neuroscience 26.14, pages 3697-3712.

Cisek, P. and J. F. Kalaska (2005). "Neural correlates of reaching decisions in dorsal premotor cortex: specification of multiple direction choices and final selection of action". In: Neuron 45.5, pages 801-814.

Clark, G. and J. W. Ward (1948). "Responses elicited from the cortex of monkeys by electrical stimulation through fixed electrodes". In: Brain 71.3, pages 332-342.

Cohen, D. (1968). "Magnetoencephalography: evidence of magnetic fields produced by alpha-rhythm currents". In: Science 161.3843, pages 784-786. 
Collinger, J. L., B. Wodlinger, J. E. Downey, W. Wang, E. C. Tyler-Kabara, D. J. Weber, A. J. McMorland, M. Velliste, M. L. Boninger, and A. B. Schwartz (2013). "High-performance neuroprosthetic control by an individual with tetraplegia". In: The Lancet 381.9866, pages 557-564.

Cooper, S. and D. Denny-Brown (1927). "Responses to stimulation of the motor area of the cerebral cortex". In: Proceedings of the Royal Society of London. Series B, Containing Papers of a Biological Character 102.716, pages 222-236.

Council of European Union (2010). "DIRECTIVE 2010/63/EU OF THE EUROPEAN PARLIAMENT AND OF THE COUNCIL of 22 September 2010 on the protection of animals used for scientific purposes". In: OJ L 276, pages 33-79.

Dannenbaum, R. M. and R. W. Dykes (1988). "Sensory loss in the hand after sensory stroke: therapeutic rationale". In: Archives of physical medicine and rehabilitation 69.10, pages 833-839.

Dayan, P., L. Abbott, et al. (2003). "Theoretical neuroscience: computational and mathematical modeling of neural systems". In: Journal of Cognitive Neuroscience 15.1, pages 154-155.

Decety, J., D. Perani, M. Jeannerod, V. Bettinardi, B. Tadary, R. Woods, J. C. Mazziotta, and F. Fazio (1994). "Mapping motor representations with positron emission tomography". In: Nature 371.6498, page 600.

Delhaye, B. P., K. H. Long, and S. J. Bensmaia (2018). "Neural basis of touch and proprioception in primate cortex". In: Comprehensive Physiology 8.4, pages 1575-1602.

Dubner, R., R. Sumino, and W. I. Wood (1975). "A peripheral" cold" fiber population responsive to innocuous and noxious thermal stimuli applied to monkey's face". In: Journal of neurophysiology 38.6, pages 1373-1389.

Dunbar, R. I. (1991). "Functional significance of social grooming in primates". In: Folia primatologica 57.3, pages 121-131.

Edell, D. J., V. V. Toi, V. M. McNeil, and L. Clark (1992). "Factors influencing the biocompatibility of insertable silicon microshafts in cerebral cortex". In: IEEE Transactions on Biomedical Engineering 39.6, pages 635-643.

Edin, B. B. (1992). "Quantitative analysis of static strain sensitivity in human mechanoreceptors from hairy skin". In: Journal of neurophysiology 67.5, pages 1105-1113.

- (2001). "Cutaneous afferents provide information about knee joint movements in humans". In: The Journal of physiology 531.1, pages 289-297.

- (2004). "Quantitative analyses of dynamic strain sensitivity in human skin mechanoreceptors". In: Journal of neurophysiology 92.6, pages 3233-3243.

Evarts, E. V. (1968). "Relation of pyramidal tract activity to force exerted during voluntary movement." In: Journal of neurophysiology 31.1, pages 14-27. 
Feix, T., I. M. Bullock, and A. M. Dollar (2014a). "Analysis of human grasping behavior: Correlating tasks, objects and grasps". In: IEEE transactions on haptics 7.4, pages 430441.

- (2014b). "Analysis of human grasping behavior: Object characteristics and grasp type". In: IEEE transactions on haptics 7.3, pages 311-323.

Feng, L. C., T. J. Howell, and P. C. Bennett (2016). "How clicker training works: comparing reinforcing, marking, and bridging hypotheses". In: Applied Animal Behaviour Science 181, pages 34-40.

Fernald, R. D. (2006). "Casting a genetic light on the evolution of eyes". In: Science 313.5795, pages 1914-1918.

Fetz, E., D. Finocchio, M. Baker, and M. Soso (1980). "Sensory and motor responses of precentral cortex cells during comparable passive and active joint movements". In: Journal of Neurophysiology 43.4, pages 1070-1089.

Filimon, F., J. D. Nelson, R.-S. Huang, and M. I. Sereno (2009). "Multiple parietal reach regions in humans: cortical representations for visual and proprioceptive feedback during on-line reaching". In: Journal of Neuroscience 29.9, pages 2961-2971.

Flamary, R. and A. Rakotomamonjy (2012). "Decoding finger movements from ECoG signals using switching linear models". In: Frontiers in neuroscience 6, page 29.

Flesher, S. N., J. L. Collinger, S. T. Foldes, J. M. Weiss, J. E. Downey, E. C. Tyler-Kabara, S. J. Bensmaia, A. B. Schwartz, M. L. Boninger, and R. A. Gaunt (2016). "Intracortical microstimulation of human somatosensory cortex". In: Science translational medicine 8.361, 361ra141-361ra141.

Fluet, M.-C., M. A. Baumann, and H. Scherberger (2010). "Context-specific grasp movement representation in macaque ventral premotor cortex". In: Journal of Neuroscience 30.45 , pages 15175-15184.

Fogassi, L., V. Raos, G. Franchi, V. Gallese, G. Luppino, and M. Matelli (1999). "Visual responses in the dorsal premotor area F2 of the macaque monkey". In: Experimental brain research 128.1-2, pages 194-199.

Fogassi, L., V. Gallese, L. Fadiga, G. Luppino, M. Matelli, and G. Rizzolatti (1996). "Coding of peripersonal space in inferior premotor cortex (area F4)". In: Journal of neurophysiology 76.1, pages 141-157.

Foldes, S. T., D. J. Weber, and J. L. Collinger (2015). "MEG-based neurofeedback for hand rehabilitation". In: Journal of neuroengineering and rehabilitation 12.1, page 85.

Fritsch, G. and E. Hitzig (1870). "Ueber die elektrische Erregbarkeit des Grosshirns". In: Arch, anat. Physiol. Wiss. Med. 37, pages 300-332.

Gallese, V., A. Murata, M. Kaseda, N. Niki, and H. Sakata (1994). "Deficit of hand preshaping after muscimol injection in monkey parietal cortex." In: Neuroreport: An International Journal for the Rapid Communication of Research in Neuroscience. 
Georgopoulos, A. P., J. F. Kalaska, R. Caminiti, and J. T. Massey (1982). "On the relations between the direction of two-dimensional arm movements and cell discharge in primate motor cortex". In: Journal of Neuroscience 2.11, pages 1527-1537.

Gibson, J. J. (1958). "Visually controlled locomotion and visual orientation in animals". In: British journal of psychology 49.3, pages 182-194.

Gillis, T. E., A. C. Janes, and M. J. Kaufman (2012). "Positive reinforcement training in squirrel monkeys using clicker training". In: American journal of primatology 74.8, pages 712-720.

Gray, C. M., P. E. Maldonado, M. Wilson, and B. McNaughton (1995). "Tetrodes markedly improve the reliability and yield of multiple single-unit isolation from multi-unit recordings in cat striate cortex". In: Journal of neuroscience methods 63.1-2, pages 4354.

Graziano, M. S., C. S. Taylor, and T. Moore (2002). "Complex movements evoked by microstimulation of precentral cortex". In: Neuron 34.5, pages 841-851.

Griffin, D. R., F. A. Webster, and C. R. Michael (1960). "The echolocation of flying insects by bats". In: Animal behaviour 8.3-4, pages 141-154.

Grigg, P. (1994). "Peripheral neural mechanisms in proprioception". In: Journal of Sport Rehabilitation 3.1, pages 2-17.

Hall, E. L., S. E. Robson, P. G. Morris, and M. J. Brookes (2014). "The relationship between MEG and fMRI". In: Neuroimage 102, pages 80-91.

Haridas, M. and M. A. Malangoni (2008). "Predictive factors for surgical site infection in general surgery". In: Surgery 144.4, pages 496-503.

Harris, J. P. and D. J. Tyler (2013). "Biological, mechanical, and technological considerations affecting the longevity of intracortical electrode recordings". In: Critical Reviews in Biomedical Engineering 41.6.

Hasler, A. D. (1954). "Odour perception and orientation in fishes". In: Journal of the Fisheries Board of Canada 11.2, pages 107-129.

Hatsopoulos, N. G. and A. J. Suminski (2011). "Sensing with the motor cortex". In: Neuron 72.3, pages 477-487.

Hepp-Reymond, M.-C., E. J. Hüsler, M. A. Maier, and H.-X. Qi (1994). "Force-related neuronal activity in two regions of the primate ventral premotor cortex". In: Canadian journal of physiology and pharmacology 72.5, pages 571-579.

Hochberg, L. R., M. D. Serruya, G. M. Friehs, J. A. Mukand, M. Saleh, A. H. Caplan, A. Branner, D. Chen, R. D. Penn, and J. P. Donoghue (2006). "Neuronal ensemble control of prosthetic devices by a human with tetraplegia". In: Nature 442.7099, page 164.

Hoshi, E. and J. Tanji (2000). "Integration of target and body-part information in the premotor cortex when planning action". In: Nature 408.6811, page 466.

Hubel, D. H. and T. N. Wiesel (1959). "Receptive fields of single neurones in the cat's striate cortex". In: The Journal of physiology 148.3, pages 574-591. 
Hulliger, M., E. Nordh, A. Thelin, and A. Vallbo (1979). "The responses of afferent fibres from the glabrous skin of the hand during voluntary finger movements in man." In: The Journal of Physiology 291.1, pages 233-249.

Hyvärinen, J. and A. Poranen (1978). "Receptive field integration and submodality convergence in the hand area of the post-central gyrus of the alert monkey." In: The Journal of Physiology 283.1, pages 539-556.

Im, C. and J.-M. Seo (2016). "A review of electrodes for the electrical brain signal recording". In: Biomedical Engineering Letters 6.3, pages 104-112.

Iwamura, Y., M. Tanaka, M. Sakamoto, and O. Hikosaka (1983). "Functional subdivisions representing different finger regions in area 3 of the first somatosensory cortex of the conscious monkey". In: Exp Brain Res 51.3, pages 315-326.

- (1985). "Diversity in receptive field properties of vertical neuronal arrays in the crown of the postcentral gyrus of the conscious monkey". In: Experimental brain research 58.2, pages 400-411.

Jeannerod, M., M. A. Arbib, G. Rizzolatti, and H. Sakata (1995). "Grasping objects: the cortical mechanisms of visuomotor transformation". In: Trends in neurosciences 18.7, pages 314-320.

Johansson, R. (1991). "How is grasping modified by somatosensory input". In: Motor control: Concepts and issues 14, page 331.

Johnson, K. O. (2001). "The roles and functions of cutaneous mechanoreceptors". In: Current opinion in neurobiology 11.4, pages 455-461.

Johnson, K. O. and S. S. Hsiao (1992). "Neural mechanisms of tactual form and texture perception". In: Annual review of neuroscience 15.1, pages 227-250.

Kaas, J. H., R. J. Nelson, M. Sur, C.-S. Lin, and M. M. Merzenich (1979). "Multiple representations of the body within the primary somatosensory cortex of primates". In: Science 204.4392, pages 521-523.

Kakei, S., D. S. Hoffman, and P. L. Strick (1999). "Muscle and movement representations in the primary motor cortex". In: Science 285.5436, pages 2136-2139.

Kell, C. A., K. von Kriegstein, A. Rösler, A. Kleinschmidt, and H. Laufs (2005). "The sensory cortical representation of the human penis: revisiting somatotopy in the male homunculus". In: Journal of Neuroscience 25.25, pages 5984-5987.

Kim, S. S., M. Gomez-Ramirez, P. H. Thakur, and S. S. Hsiao (2015). "Multimodal interactions between proprioceptive and cutaneous signals in primary somatosensory cortex". In: Neuron 86.2, pages 555-566.

Kossen, R. (2019). "Thermosensory Transduction Mechanisms in Drosophila melanogaster". PhD thesis. Georg-August-Universität Göttingen.

Kraskov, A., H. Stögbauer, and P. Grassberger (2004). "Estimating mutual information". In: Physical review E 69.6, page 066138. 
Krubitzer, L. and M. Baldwin (2017). "Revisiting Kaas and colleagues-the homunculus: the discovery of multiple representations within the "primary" somatosensory cortex". In: Revisiting the Classic Studies in Behavioral Neuroscience, pages 33-54.

Krubitzer, L., K. J. Huffman, E. Disbrow, and G. Recanzone (2004). "Organization of area 3a in macaque monkeys: contributions to the cortical phenotype". In: Journal of Comparative Neurology 471.1, pages 97-111.

Kurata, K. and J. Tanji (1986). "Premotor cortex neurons in macaques: activity before distal and proximal forelimb movements". In: Journal of Neuroscience 6.2, pages 403-411.

Land, M. F. and R. D. Fernald (1992). "The evolution of eyes". In: Annual review of neuroscience 15.1, pages 1-29.

Laule, G. E., M. A. Bloomsmith, and S. J. Schapiro (2003). "The use of positive reinforcement training techniques to enhance the care, management, and welfare of primates in the laboratory". In: Journal of applied animal welfare science 6.3, pages 163-173.

Lehmann, S. J. and H. Scherberger (2013). "Reach and gaze representations in macaque parietal and premotor grasp areas". In: Journal of Neuroscience 33.16, pages 70387049.

Lemon, R. (2008). "An enduring map of the motor cortex." In: Experimental Physiology 93.7, page 798 .

Lieber, J. D. and S. J. Bensmaia (2019). "High-dimensional representation of texture in somatosensory cortex of primates". In: Proceedings of the National Academy of Sciences 116.8, pages 3268-3277.

Logothetis, N. K. and D. L. Sheinberg (1996). "Visual object recognition". In: Annual review of neuroscience 19.1, pages 577-621.

Luppino, G., A. Murata, P. Govoni, and M. Matelli (1999). "Largely segregated parietofrontal connections linking rostral intraparietal cortex (areas AIP and VIP) and the ventral premotor cortex (areas F5 and F4)". In: Experimental Brain Research 128.1-2, pages 181-187.

Marg, E. and J. E. Adams (1967). "Indwelling multiple micro-electrodes in the brain". In: Electroencephalography and clinical neurophysiology 23.3, pages 277-280.

McFarland, D. J., W. A. Sarnacki, and J. R. Wolpaw (2010). "Electroencephalographic (EEG) control of three-dimensional movement". In: Journal of neural engineering 7.3, page 036007.

McNaughton, B. L., J. O'Keefe, and C. A. Barnes (1983). "The stereotrode: a new technique for simultaneous isolation of several single units in the central nervous system from multiple unit records". In: Journal of neuroscience methods 8.4, pages 391-397.

Menz, V. K., S. Schaffelhofer, and H. Scherberger (2015). "Representation of continuous hand and arm movements in macaque areas M1, F5, and AIP: a comparative decoding study". In: Journal of neural engineering 12.5, page 056016. 
Michaels, J. A., B. Dann, R. W. Intveld, and H. Scherberger (2015). "Predicting reaction time from the neural state space of the premotor and parietal grasping network". In: Journal of Neuroscience 35.32, pages 11415-11432.

Miller, K. J., G. Schalk, E. E. Fetz, M. den Nijs, J. G. Ojemann, and R. P. Rao (2010). "Cortical activity during motor execution, motor imagery, and imagery-based online feedback". In: Proceedings of the National Academy of Sciences 107.9, pages 4430-4435.

Mott, F. W. and C. S. Sherrington (1985). "VIII. Experiments upon the influence of sensory nerves upon movement and nutrition of the limbs. Preliminary communication". In: Proceedings of the Royal Society of London 57.340-346, pages 481-488.

Munk, H. (1890). Ueber die Funktionen der Grosshirnrinde. Verlag von August Hirschwald. Murata, A., L. Fadiga, L. Fogassi, V. Gallese, V. Raos, and G. Rizzolatti (1997). "Object representation in the ventral premotor cortex (area F5) of the monkey". In: Journal of neurophysiology 78.4, pages 2226-2230.

Murata, A., V. Gallese, G. Luppino, M. Kaseda, and H. Sakata (2000). "Selectivity for the shape, size, and orientation of objects for grasping in neurons of monkey parietal area AIP". In: Journal of neurophysiology 83.5, pages 2580-2601.

Musallam, S., M. J. Bak, P. R. Troyk, and R. A. Andersen (2007). "A floating metal microelectrode array for chronic implantation". In: Journal of neuroscience methods 160.1 , pages 122-127.

Musial, P., S. Baker, G. Gerstein, E. King, and J. Keating (2002). "Signal-to-noise ratio improvement in multiple electrode recording". In: Journal of neuroscience methods 115.1, pages 29-43.

Mussa-Ivaldi, F. (1988). "Do neurons in the motor cortex encode movement direction? An alternative hypothesis". In: Neuroscience letters 91.1, pages 106-111.

Napier, J. (1962). "The evolution of the hand". In: Scientific American 207.6, pages 56-65. Napier, J. R. (1956). "The prehensile movements of the human hand". In: The Journal of bone and joint surgery. British volume 38.4, pages 902-913.

National Centre for the Replacement, Refinement and Reduction of Animals in Research (2017). NC3Rs Guidelines: Non-human primate accommodation, care and use.

National Research Council (2003). Guidelines for the care and use of mammals in neuroscience and behavioral research. National Academies Press.

Nelson, R., M. Sur, D. Felleman, and J. Kaas (1980). "Representations of the body surface in postcentral parietal cortex of Macaca fascicularis". In: Journal of Comparative Neurology 192.4, pages 611-643.

Nicolelis, M. A., D. Dimitrov, J. M. Carmena, R. Crist, G. Lehew, J. D. Kralik, and S. P. Wise (2003). "Chronic, multisite, multielectrode recordings in macaque monkeys". In: Proceedings of the National Academy of Sciences 100.19, pages 11041-11046. 
Oddo, C. M., L. Beccai, N. Vitiello, H. B. Wasling, J. Wessberg, and M. C. Carrozza (2011). "A mechatronic platform for human touch studies". In: Mechatronics 21.3, pages 604613.

Paul, R. L., M. Merzenich, and H. Goodman (1972). "Representation of slowly and rapidly adapting cutaneous mechanoreceptors of the hand in Brodmann's areas 3 and 1 of Macaca mulatta". In: Brain research 36.2, pages 229-249.

Peier, A. M., A. Moqrich, A. C. Hergarden, A. J. Reeve, D. A. Andersson, G. M. Story, T. J. Earley, I. Dragoni, P. McIntyre, S. Bevan, et al. (2002). "A TRP channel that senses cold stimuli and menthol". In: Cell 108.5, pages 705-715.

Penfield, W. and E. Boldrey (1937). "Somatic motor and sensory representation in the cerebral cortex of man as studied by electrical stimulation". In: Brain 60.4, pages 389443.

Penfield, W. and T. Rasmussen (1950). "The cerebral cortex of man; a clinical study of localization of function." In:

Pistohl, T., T. Ball, A. Schulze-Bonhage, A. Aertsen, and C. Mehring (2008). "Prediction of arm movement trajectories from ECoG-recordings in humans". In: Journal of neuroscience methods 167.1, pages 105-114.

Polikov, V. S., P. A. Tresco, and W. M. Reichert (2005). "Response of brain tissue to chronically implanted neural electrodes". In: Journal of neuroscience methods 148.1, pages 1-18.

Pons, T., P. Garraghty, C. Cusick, and J. Kaas (1985). "The somatotopic organization of area 2 in macaque monkeys". In: Journal of Comparative Neurology 241.4, pages 445-466.

Raos, V., G. Franchi, V. Gallese, and L. Fogassi (2003). "Somatotopic organization of the lateral part of area F2 (dorsal premotor cortex) of the macaque monkey". In: Journal of Neurophysiology 89.3, pages 1503-1518.

Raos, V., M.-A. Umiltá, V. Gallese, and L. Fogassi (2004). "Functional properties of grasping-related neurons in the dorsal premotor area F2 of the macaque monkey". In: Journal of neurophysiology 92.4, pages 1990-2002.

Raos, V., M.-A. Umiltá, A. Murata, L. Fogassi, and V. Gallese (2006). "Functional properties of grasping-related neurons in the ventral premotor area F5 of the macaque monkey". In: Journal of neurophysiology 95.2, pages 709-729.

Riehle, A. and J. Requin (1989). "Monkey primary motor and premotor cortex: singlecell activity related to prior information about direction and extent of an intended movement". In: Journal of neurophysiology 61.3, pages 534-549.

Rizzolatti, G., R. Camarda, L. Fogassi, M. Gentilucci, G. Luppino, and M. Matelli (1988). "Functional organization of inferior area 6 in the macaque monkey". In: Experimental brain research 71.3, pages 491-507.

Rizzolatti, G., L. Fadiga, V. Gallese, and L. Fogassi (1996). "Premotor cortex and the recognition of motor actions". In: Cognitive brain research 3.2, pages 131-141. 
Rizzolatti, G., L. Fogassi, and V. Gallese (2002). "Motor and cognitive functions of the ventral premotor cortex". In: Current opinion in neurobiology 12.2, pages 149-154.

Rizzolatti, G. and G. Luppino (2001). "The cortical motor system". In: Neuron 31.6, pages 889-901.

Rizzolatti, G., G. Luppino, and M. Matelli (1998). "The organization of the cortical motor system: new concepts". In: Electroencephalography and clinical neurophysiology 106.4, pages 283-296.

Rizzolatti, G., C. Scandolara, M. Matelli, and M. Gentilucci (1981). "Afferent properties of periarcuate neurons in macaque monkeys. II. Visual responses". In: Behavioural brain research 2.2, pages 147-163.

Rothemund, Y., H.-X. Qi, C. E. Collins, and J. H. Kaas (2002). "The genitals and gluteal skin are represented lateral to the foot in anterior parietal somatosensory cortex of macaques". In: Somatosensory \& motor research 19.4, pages 302-315.

Saal, H. P. and S. J. Bensmaia (2014). "Touch is a team effort: interplay of submodalities in cutaneous sensibility". In: Trends in neurosciences 37.12, pages 689-697.

Sakata, H., M. Taira, A. Murata, and S. Mine (1995). "Neural mechanisms of visual guidance of hand action in the parietal cortex of the monkey". In: Cerebral Cortex 5.5, pages 429-438.

Salas, M. A., L. Bashford, S. Kellis, M. Jafari, H. Jo, D. Kramer, K. Shanfield, K. Pejsa, B. Lee, C. Y. Liu, and R. A. Andersen (2018). "Proprioceptive and cutaneous sensations in humans elicited by intracortical microstimulation". In: Elife 7, e32904.

Salvini-Plawen, L. von and E. Mayr (1977). On the Evolution of Photorecptors and Eyes. Plenum Press.

Schaffelhofer, S. and H. Scherberger (2012). "A new method of accurate hand-and armtracking for small primates". In: Journal of neural engineering 9.2, page 026025.

Schaffelhofer, S. (2014). "From vision to action: Hand representations in macaque grasping areas AIP, F5, and M1". PhD thesis. Niedersächsische Staats-und Universitätsbibliothek Göttingen.

Schaffelhofer, S., A. Agudelo-Toro, and H. Scherberger (2015). "Decoding a wide range of hand configurations from macaque motor, premotor, and parietal cortices". In: Journal of Neuroscience 35.3, pages 1068-1081.

Schaffelhofer, S. and H. Scherberger (2016). "Object vision to hand action in macaque parietal, premotor, and motor cortices". In: Elife 5, e15278.

Schieber, M. H. and L. S. Hibbard (1993). "How somatotopic is the motor cortex hand area?" In: Science 261.5120, pages 489-492.

Schlegel, P., S. Steinfartz, and B. Bulog (2009). "Non-visual sensory physiology and magnetic orientation in the Blind Cave Salamander, Proteus anguinus (and some other cave-dwelling urodele species). Review and new results on light-sensitivity 
and non-visual orientation in subterranean urodeles (Amphibia)". In: Animal biology 59.3, pages 351-384.

Schmidt, S., K. Horch, and R. Normann (1993). "Biocompatibility of silicon-based electrode arrays implanted in feline cortical tissue". In: Journal of biomedical materials research 27.11, pages 1393-1399.

Schroeder, K. E., Z. T. Irwin, A. J. Bullard, D. E. Thompson, J. N. Bentley, W. C. Stacey, P. G. Patil, and C. A. Chestek (2017). "Robust tactile sensory responses in finger area of primate motor cortex relevant to prosthetic control". In: Journal of neural engineering 14.4, page 046016.

Seelke, A. M., J. J. Padberg, E. Disbrow, S. M. Purnell, G. Recanzone, and L. Krubitzer (2011). "Topographic maps within Brodmann's area 5 of macaque monkeys". In: Cerebral Cortex 22.8, pages 1834-1850.

Sejnowski, T. J., P. S. Churchland, and J. A. Movshon (2014). "Putting big data to good use in neuroscience". In: Nature neuroscience 17.11, page 1440.

Sergio, L. E., C. Hamel-Pâquet, and J. F. Kalaska (2005). "Motor cortex neural correlates of output kinematics and kinetics during isometric-force and arm-reaching tasks". In: Journal of neurophysiology 94.4, pages 2353-2378.

Simeral, J., S.-P. Kim, M. Black, J. Donoghue, and L. Hochberg (2011). "Neural control of cursor trajectory and click by a human with tetraplegia 1000 days after implant of an intracortical microelectrode array". In: Journal of neural engineering 8.2, page 025027.

Skinner, B. F. (1990). The behavior of organisms: An experimental analysis. BF Skinner Foundation.

Smeets, J. B. and E. Brenner (1999). "A new view on grasping". In: Motor control 3.3, pages 237-271.

Srinivasan, R. (1999). "Methods to improve the spatial resolution of EEG". In: International Journal of Bioelectromagnetism 1.1, pages 102-111.

Stensaas, S. S. and L. Stensaas (1978). "Histopathological evaluation of materials implanted in the cerebral cortex". In: Acta neuropathologica 41.2, pages 145-155.

Stephan, K., G. Fink, R. Passingham, D. Silbersweig, A. Ceballos-Baumann, C. Frith, and R. Frackowiak (1995). "Functional anatomy of the mental representation of upper extremity movements in healthy subjects". In: Journal of neurophysiology 73.1, pages 373-386.

Stone, K. D. and C. L. Gonzalez (2014). "Grasping without sight: insights from the congenitally blind". In: PloS one 9.10, e110175.

Suminski, A. J., D. C. Tkach, and N. G. Hatsopoulos (2009). "Exploiting multiple sensory modalities in brain-machine interfaces". In: Neural networks 22.9, pages 1224-1234.

Tabot, G. A., J. F. Dammann, J. A. Berg, F. V. Tenore, J. L. Boback, R. J. Vogelstein, and S. J. Bensmaia (2013). "Restoring the sense of touch with a prosthetic hand through a brain 
interface". In: Proceedings of the National Academy of Sciences 110.45, pages 1827918284.

Taira, M., S. Mine, A. Georgopoulos, A. Murata, and H. Sakata (1990). "Parietal cortex neurons of the monkey related to the visual guidance of hand movement". In: Experimental brain research 83.1, pages 29-36.

Tanaka, K., K. Matsunaga, and H. O. Wang (2005). "Electroencephalogram-based control of an electric wheelchair". In: IEEE transactions on robotics 21.4, pages 762-766.

Terry, R. L. (1970). "Primate grooming as a tension reduction mechanism". In: The Journal of Psychology 76.1, pages 129-136.

TierSchG (1972, last adapted 2018). Tierschutzgesetz, in der Fassung der Bekanntmachung vom 18. Mai 2006 BGBl.IS.1206, 1313, das zuletzt durch Artikel 1 des Gesetzes vom 17. Dezember 2018 BGBl.IS.2586 geändert worden ist, TierSchG. URL: https : //www . gesetze-im-internet.de/tierschg/BJNR012770972.html.

TierSchVersV (2013, last adapted 2015). Tierschutz-Versuchstierverordnung vom 1. August 2013 (BGBI. I S. 3125, 3126), die zuletzt durch Artikel 394 der Verordnung vom 31. August 2015 (BGBI. I S. 1474) geändert worden ist, (TierSchVersV). URL: https : / www . gesetze-im-internet. de/tierschversv/BJNR312600013.html.

Todorov, E. (2000). "Direct cortical control of muscle activation in voluntary arm movements: a model". In: Nature neuroscience 3.4, page 391.

Truccolo, W., G. M. Friehs, J. P. Donoghue, and L. R. Hochberg (2008). "Primary motor cortex tuning to intended movement kinematics in humans with tetraplegia". In: Journal of Neuroscience 28.5, pages 1163-1178.

Turner, J., W. Shain, D. Szarowski, M. Andersen, S. Martins, M. Isaacson, and H. Craighead (1999). "Cerebral astrocyte response to micromachined silicon implants". In: Experimental neurology 156.1, pages 33-49.

Umilta, M. A., T. Brochier, R. L. Spinks, and R. N. Lemon (2007). "Simultaneous recording of macaque premotor and primary motor cortex neuronal populations reveals different functional contributions to visuomotor grasp". In: Journal of neurophysiology 98.1, pages 488-501.

Van Essen, D. C. (2003). "Organization of visual areas in macaque and human cerebral cortex". In: The visual neurosciences 1, pages 507-521.

Van Gerven, M., J. Farquhar, R. Schaefer, R. Vlek, J. Geuze, A. Nijholt, N. Ramsey, P. Haselager, L. Vuurpijl, S. Gielen, and P. Desain (2009). "The brain-computer interface cycle". In: Journal of neural engineering 6.4, page 041001.

Velliste, M., S. Perel, M. C. Spalding, A. S. Whitford, and A. B. Schwartz (2008). "Cortical control of a prosthetic arm for self-feeding". In: Nature 453.7198, page 1098.

Vogt, C. (1919). "Allgemeinere ergebnisse unserer hirnforschung". In: J Psychol Neurol (Leipz) 25, pages 279-461. 
Von Humboldt, A. and A. Bonpland (1811). Recueil d'observations de zoologie et d'anatomie comparée: faites dans l'Océan Atlantique, dans l'interieur du nouveau continent et dans la Mer du Sud, pendant les années 1799, 1800, 1801, 1802 et 1803. F. Schoell et G. Dufour.

Vriens, J., B. Nilius, and T. Voets (2014). "Peripheral thermosensation in mammals". In: Nature Reviews Neuroscience 15.9, page 573.

Walsh, L. D., J. L. Taylor, and S. C. Gandevia (2014). "Proprioceptive mechanisms and the human hand". In: The Human Hand as an Inspiration for Robot Hand Development. Springer, pages 123-141.

Weber, A. I., H. P. Saal, J. D. Lieber, J.-W. Cheng, L. R. Manfredi, J. F. Dammann, and S. J. Bensmaia (2013). "Spatial and temporal codes mediate the tactile perception of natural textures". In: Proceedings of the National Academy of Sciences 110.42, pages 17107-17112.

Weinrich, M., S. Wise, and K.-H. Mauritz (1984). "A neurophysiological study of the premotor cortex in the rhesus monkey". In: Brain 107.2, pages 385-414.

Wessberg, J., C. R. Stambaugh, J. D. Kralik, P. D. Beck, M. Laubach, J. K. Chapin, J. Kim, S. J. Biggs, M. A. Srinivasan, and M. A. Nicolelis (2000). "Real-time prediction of hand trajectory by ensembles of cortical neurons in primates". In: Nature 408.6810, page 361.

Wheat, H. E., L. M. Salo, and A. W. Goodwin (2004). "Human ability to scale and discriminate forces typical of those occurring during grasp and manipulation". In: Journal of Neuroscience 24.13, pages 3394-3401.

Wodlinger, B., J. Downey, E. Tyler-Kabara, A. Schwartz, M. Boninger, and J. Collinger (2014). "Ten-dimensional anthropomorphic arm control in a human brain- machine interface: difficulties, solutions, and limitations". In: Journal of neural engineering 12.1, page 016011.

Woolsey, C. N. and D. Fairman (1946). "Contralateral, ipsilateral, and bilateral representation of cutaneous receptors in somatic areas I and II of the cerebral cortex of pig, sheep, and other mammals". In: Surgery 19.5, pages 684-702.

Zimmermann, E. A. W. von (1780). Geographische Geschichte des Menschen, und der allgemein verbreiteten vierfüssigen Thiere: nebst einer hieher gehörigen zoologischen Weltcharte. Volume 1. in der Weygandschen Buchhandlung. 


\section{Chapter 7}

\section{Acknowledgements}

Writing a thesis is never the effort of a single person, therefore I would like to thank the following people for support, input and help.

First of all, I thank Hans for supervising and mentoring me these past four years and for introducing me to the field of primate neurophysiology and giving me the opportunity to work with these incredible animals. I'd also like to thank my thesis committee, Ralf and Igor, for supporting this project with their insights and advice during meetings and of course my examination board members Prof. Dr. Manuela Schmidt, Prof. Dr. Tim Gollisch and Prof. Dr. Alexander Gail.

Thank you Sliman, for many discussions and advice about my project and academia in general.

Thank you to the Hühnerstall, Sabine, Natalie and Ricarda for their support with animal training and for keeping me good company. Hanging out with you is always fun. You were the first to introduce me to monkey handling and training, had ideas about task designs and how to teach the monkeys and I am very grateful for what you taught me. Also, thank you Cara. Observing the monkeys with you is always fun.

I'd also like to thank Matthias and Timo for technical support and discussions about the best setup materials. I could always rely on you to find a solution for all ideas I had. I also thank Onur for helping to build the newest generation of data glove.

Thank you Andres and Andrej for technical support with the setup, computers and the data glove.

I want to thank Ben for taking time to introduce me to all aspects of this field, be it what to consider when building a setup or data analysis methods. I could always come to you when I had questions about anything.

Thank you Swathi, for being a good friend and for always listening if I want to discuss something. You always take time to bounce ideas back and forth or just to listen to random stuff I wanted to talk about. 
I'd like to thank Jeroen for being the first to introduce me to monkey work. Meeting Zara and seeing how well you two worked together helped me making the decision to start working in this field and thank you for your advice on setup building.

Thank you Anne-Do, for many discussions about the turntable setup and tasks.

I'd also like to thank the other current and former lab members, for creating an enviorment that is fun to work in, for help with analyses and fruitful discussions. Thank you Rijk, Katharina, Jonathan, Wei-An, James and Stefan for contributing to this great lab atmosphere.

Thank you Kerstin, Ricarda and Anja, for always organising everything and making sure that I had all materials and ressources I needed.

I furthermore thank Prof. Dr. Calogero Oddo and Dr. Domenico Camboni from the Scuola Superiore Sant'Anna in Italy for borrowing their stimulation platform to us and providing technical support, which allowed me to conduct the passive stimulation experiment in this thesis.

I'd like to thank the SFB889, GTPN program and the SMN program of the GGNB for financial and educational support.

Special thanks go out to not only Homer and Ralph, for being good boys, but also to the first monkeys I ever got into contact with, Zara and Moe.

Thank you to Nele, for helping me improving drawings and just generally sharing many laughs with me. Thank you to the Howrse-Dreamteam for supporting my efforts (through three degrees!), listing to my problems and many hours of fun distractions.

I thank my parents and my grandma for supporting and encouraging my studies, always believing in me and showing interest in my work. I thank my brother Tobias, with whom I share many memories that sparked my interest in Biology, like when we held snail races in our grandparents's garden or roamed through the Ochsendorfer Wald.

Robert $8(I)$, thank you for always being there for me and for now walking this path with me. Thank you for supporting me during my Bachelor, Master and now PhD and for now proofreading the third thesis. Thank you for always picking me up when I'm down.

Thank you to anyone I stupidly forgot. I am very grateful for everything. 